\title{
The Solar System
}

\section{Nature and mechanics}

\author{
Mario Ljubičić (Amenoum)
}

mljubicic99 @ gmail.com (ORCID: 0000-0001-9400-0551)

\begin{abstract}
(short):
The origin, mechanics and properties of the Solar System are described in the framework of Complete Relativity theory by Amenoum.
\end{abstract}

Keywords: complete relativity, solar system. 


\section{Abstract}

The origin, mechanics and properties of the Solar System are described in the framework of Complete Relativity ${ }^{1}$ (CR).

Main conclusions are:

- Solar System is a scaled Carbon isotope with a nucleus in a condensed (bosonic) state

- life is common everywhere, life on Earth is not special even in the Solar System

- Earth is a conscious living being and a closest best match for a supernatural phenomena often referred to as god (there are no absolute, all-mighty gods)

- higher intelligence is all around us, human intelligence is mostly polarized and forms only one part of the intelligence spectrum

- climate change is only a part (trigger from one perspective) of bigger global changes on Earth and in the Solar System

- massive extinction event on Earth is imminent 


\section{Introduction}

Here I hypothesize that the Solar System is a large scale ${ }^{10} \mathrm{C}$ atom (10-Carbon isotope) and provide evidence for the equivalence of large $\left(\mathbf{U}_{\mathbf{1}}\right)$ scale systems with standard $\left(\mathbf{U}_{\mathbf{0}}\right)$ scale systems through the analysis of the Solar System in the context of CR.

Note that ${ }^{10} \mathrm{C}$ isotope is unstable on standard scale, with a half-life of $\sim 19.3$ seconds. Its apparent stability on $U_{1}$ scale must be either the result of time dilation [due to scale difference] or inversion of stability between adjacent scales (vertical energy levels).

In case of inversion, stable systems on one scale would be unstable on the other and vice versa.

I hypothesize that the structure of planetary systems is the result of inflation of gravitational maximums from standard scale atoms, likely in the events of annihilation at event horizons.

I propose that, in this process, electro-magnetic component of the general force has been exchanged with the neutral gravitational component resulting in the dominance of gravity over electro-magnetic force at this scale.

However, I also hypothesize that such exchange is natural on standard scale - the atoms are cycling between polarized and neutral states (although durations in particular states might be inverted between scales).

Note that due to instability of ${ }^{10} \mathrm{~B}$ (decay product of ${ }^{10} \mathrm{C}$ and ${ }^{10} \mathrm{Be}$ ) at $\mathrm{U}_{1}$ scale, the Solar System must also be cycling between ${ }^{10} \mathrm{C}$ and ${ }^{10} \mathrm{Be}\left({ }^{10} \mathrm{~B}\right.$ being the intermediate state).

Implications of scale invariance of physical laws [and $\mathbf{C R}$ in general] on nature are large and some of these are further discussed and analyzed, primarily the implication on life. 


\section{Constants}

Here are the commonly used constants in the article.

The values of planetary constants are taken from NASA Planetary Fact Sheet ${ }^{2}$.

\begin{tabular}{|c|c|c|}
\hline Description & Constant & Value \\
\hline Neptune mass on scale 1 & $\mathrm{M}_{\mathrm{U}_{1}}$ & $1.02413 * 10^{\wedge} 26 \mathrm{~kg}$ \\
\hline Neptune mass on scale $\mathbf{0}$ & $\mathbf{M}_{\mathrm{U}_{0}}$ & $\begin{array}{l}\left(9.10938356 * 10^{\wedge}-31 \mathrm{~kg} / 510998.9461 \mathrm{eV}\right) *(510998.9461 \mathrm{eV}-11.260288 \mathrm{eV})= \\
9.109182827 * 10^{\wedge}-31 \mathrm{~kg}\end{array}$ \\
\hline Neptune orbital velocity & $\mathrm{Vu}_{1}$ & $5430 \mathrm{~m} / \mathrm{s}$ \\
\hline Neptune spin velocity & $\mathrm{SU}_{1}$ & $2660 \mathrm{~m} / \mathrm{s}$ \\
\hline Neptune radius on scale 1 & $\mathbf{R}_{\mathbf{U}_{1}}$ & $24622000 \mathrm{~m}$ \\
\hline Neptune radius on scale 0 & $\mathbf{R}_{\mathbf{U}_{0}}$ & $(24622000 \mathrm{~m} / 4495060000000 \mathrm{~m}){ }^{*} 70^{*} 10^{\wedge}-12 \mathrm{~m}=3.834298096 * 10^{\wedge}-16 \mathrm{~m}$ \\
\hline $\begin{array}{l}\text { Solar System charge radius }=\text { Neptune } \\
\text { orbital radius }\end{array}$ & $\mathrm{r}_{\mathrm{U}_{1}}$ & $4495060000000 \mathrm{~m}$ \\
\hline Sun mass & $\mathbf{M}_{\odot}$ & $1.988500 * 10^{\wedge} 30 \mathrm{~kg}$ \\
\hline Sun radius & $\mathbf{R}_{\odot}$ & $695735 \mathrm{~km}=695735000 \mathrm{~m}$ \\
\hline Earth mass & & $5.9723 * 10^{\wedge} 24 \mathrm{~kg}$ \\
\hline Carbon-12 atom mass & & $1.992646547^{*} 10^{\wedge}-23 \mathrm{~g}=1.992646547^{*} 10^{\wedge}-26 \mathrm{~kg}$ \\
\hline $\begin{array}{l}\text { Carbon }-12 \text { charge radius }=\text { Carbon }-10 \\
\text { charge radius }\end{array}$ & $\mathrm{r}_{\mathrm{U}_{0}}$ & $70 \mathrm{pm}=70 * 10^{\wedge}-12 \mathrm{~m}$ \\
\hline Carbon-10 nucleus charge radius & & $2.708 * 10^{\wedge}-15 m$ \\
\hline Carbon-10 nucleus mass & & $10.016853 \mathrm{u}=1.663337576 * 10^{\wedge}-26 \mathrm{~kg}$ \\
\hline Standard speed of light & $c=c_{0}$ & 2.99792458 * $10^{\wedge} 8 \mathrm{~m} / \mathrm{s}$ \\
\hline Standard electron mass & $\mathbf{M}_{\mathrm{e}}$ & $9.10938356 * 10^{\wedge}-31 \mathrm{~kg}$ \\
\hline
\end{tabular}




\section{Definitions}




\subsection{Elementary charge}

Elementary particles relative to any system of certain scale are charged.

Physical interpretation (manifestation) of charge is dependable on environment, but each charge has a neutral component (graviton), it's associated space, and electric component.

Electric component is always a composite, and generally consists of $\mathbf{2}$ quanta of identical charge (dominant) and 1 quantum of opposite (anti) charge, which are strongly entangled.

Thus, there are no absolute monopoles. The concept of dominant charge allows relative electric monopoles but relative magnetic monopoles require more complex structure as the magnetic field of (relatively) elementary charge is the result of interaction of at least two opposite electric charges and thus has two poles.

Spin momentum of charge quanta is quantized, by a relative constant $(\hbar)$ - a quantum of momentum.

Suppose the value of each spin momentum is equal to $1 / 2 \hbar$ in value, and the spins of two dominant charges are perpendicular to each other (having a [fixed] phase difference of $\pi / 2$ degrees). Two dominant charges now have a total magnetic spin momentum:

$$
S_{1}=\sqrt{\left(\frac{1}{2} \hbar\right)^{2}+\left(\frac{1}{2} \hbar\right)^{2}}=\frac{\sqrt{2}}{2} \hbar=\frac{1}{\sqrt{2}} \hbar
$$

a)

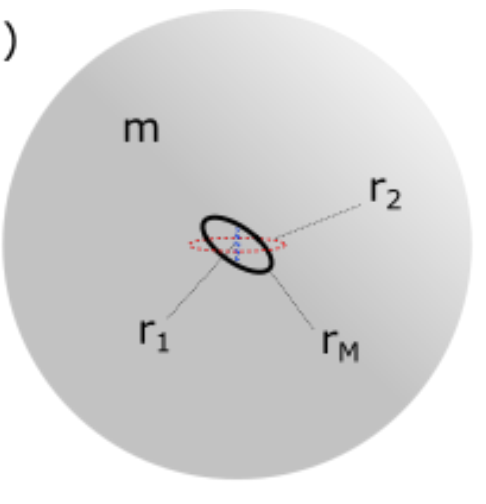

b)

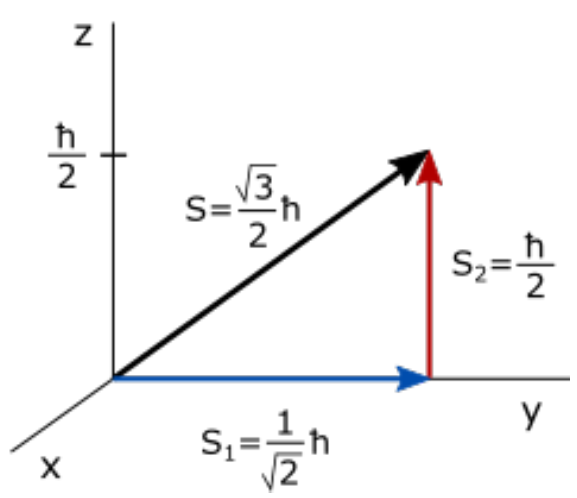

Fig. 1: Spin momentum

Total spin momentum of the particle is thus:

$$
\vec{S}=\vec{S}_{1}+\vec{S}_{2}
$$

If the $\mathbf{S}_{\mathbf{2}}$ (anti) charge momentum is perpendicular to $\mathbf{S}_{\mathbf{1}}$, the value of total spin momentum is: 


$$
S=\sqrt{\left(\frac{1}{\sqrt{2}} \hbar\right)^{2}+\left(\frac{1}{2} \hbar\right)^{2}}=\sqrt{\frac{1}{2}\left(\frac{1}{2}+1\right)} \hbar=\frac{\sqrt{3}}{2} \hbar
$$

Due to fixed $\pi / 2$ phase and equal value, the influence of the components of $\mathbf{S}_{1}$ on the orientation [of the momentum projection] cancel, and the orientation of the projection of the momentum $\mathbf{S}$ on the magnetic axis will depend solely on the orientation of momentum $\mathbf{S}_{\mathbf{2}}$.

With the applied magnetic field, the projection of the momentum on the magnetic axis (ie. z) will thus be oriented either up or down:

$$
S_{z}= \pm \frac{1}{2} \hbar
$$

Momentum $\mathbf{S}_{\mathbf{1}}$ also has two possible orientations (left, right) and it represents chirality (handedness) of a particle.

Everything in a universe has dual nature, at least. A particle can not only be interpreted as a wave, but also as a living being (and vice versa).

Spin can thus be interpreted as sex (male/female - relating to a sexual reproduction organ), chirality as gender or sexuality (homo/hetero).

A free naked charge can change these properties easily, while for interacting particles with acquired matter energy (real mass) this will be harder - proportionally to acquired matter.

Fig. 1 a) shows charge in collapsed ground state (particle) with acquired real mass $\mathbf{m}$, charge radii $\mathbf{r}_{\mathbf{1}}, \mathbf{r}_{\mathbf{2}}$ and radius of imaginary mass $\mathbf{r}_{\mathbf{M}}$.

It's momentum is quantized by $\hbar$, electric charge by $\mathbf{e}$ and gravitational force by $\hbar_{\mathbf{m g}}$. The space of such particle is characterized by $\boldsymbol{\varepsilon}$ (electric permittivity) and $\boldsymbol{\mu}$ (magnetic permeability) properties.

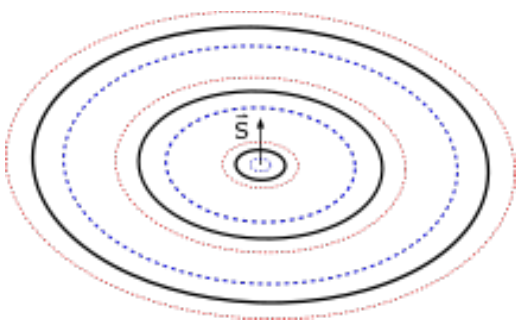

Fig. 2: Charge wave

With decreasing environmental pressure (em/gravitational field interactions) a quantum may split into smaller quanta, spreading as far as possible, but still entangled, with a wave-like distribution of potential. Fig. 2 shows such unbound, free charge. The total momentum and force is the sum of individual quanta and equal to 
original momentum and force of the particle.
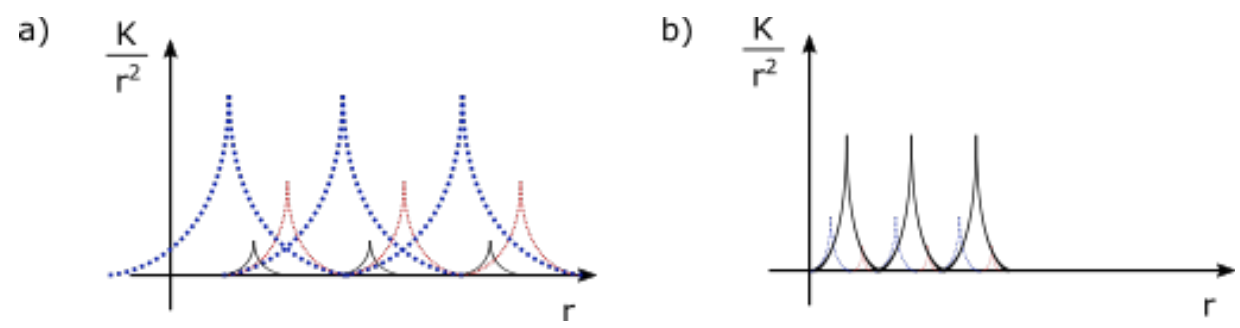

Fig. 3: Charge wave forces

Fig. 3 a) shows strength of forces of a wave with distance from center (black = gravitational force, blue and red = electric force). Now each component (maximum) of a wave, starting from outer ones, can be excited independently, can change spin, merge with adjacent maximums and form moon charges.

This allows the charge to interact (interfere) with itself, exhibiting a wave-like nature.

Fig. 3 b) shows how the space of the same particle can be modified by interaction with another particle essentially, the electric force has been exchanged for gravitational force. Such interaction may also collapse the wave into a particle with moon charges, where the number of moons depends on the equilibrium point of interaction (difference in energy of interacting particles).

Note that it is possible for the effect to be strongly localized - local space may be modified to attenuate one force and strengthen the other, while particles outside that space may not feel such [degree of] change.

\subsubsection{Equilibrium and nature of forces}

Equilibrium state of 3 components of charge is maintained through rotation. Due to rotation of local space, general force is a centripetal force and in stable orbitals equal to centrifugal force.

In case of a completely neutralized force:

$$
\frac{m v^{2}}{r}=\frac{G M m}{r^{2}}
$$

This is established when angular velocity of the orbiting body is equal to angular velocity of space (gravitational field line):

$$
v=v_{s}=\sqrt{\frac{G M}{r}}
$$

If the body increases velocity $\left(\mathbf{v}>\mathbf{v}_{\mathbf{s}}\right)$, centrifugal force becomes greater than gravitational force and now acts as a fictitious repulsive force. 
For $\mathbf{v}<\mathbf{v}_{\mathbf{s}}$, gravitational force is higher than centrifugal force, and the body feels attractive force.

Nature (polarization) of the force can thus be changed with a change in radii (expansion/collapse) of gravitational maximums.

This allows for electro-magnetic force to be a fictitious force - a result of radii change of maximums due to absorption and emission of photons.

Note that polarization of atoms is done through emission and absorption of electrons, which is affecting the atom radius - positive polarization will generally decrease radius (in common atom radius interpretation), while negative will increase it.

However, when radius is proportional to gravity, positive polarization would create repulsion, while negative would create attraction. Thus, this cannot be the equivalent of electro-magnetic force, as nature of EM force (attraction/repulsion) depends on the pair of interacting charges, not solely on the polarity of individual charge.

This can be solved through selective absorption/emission.

Assuming that negative particles emit [or their space is composed of] lower scale particles (photon electrons $=$ photon.e) while positive particles emit lower scale anti-particles (photon. $\mathrm{e}^{+}$), annihilation of photon electrons and anti-electrons at barycenter into graviton neutrinos (gravitational maximums) would create attractive force between oppositely charged particles.

Such created maximum would then attract all particles regardless of charge, unless the polarization of space is such that it is increasing the entanglement (specific wormhole) cross-section between the interacting particles - effectively making created gravity more private. Note that such wormholes are magnetic field lines and annihilation must be happening on the outer rim (event horizon) of the wormhole.

With no change in volume of the wormhole, increased cross-section must reduce the length of the wormhole - decreasing distance between entangled particles.

If the trajectory of emitted photon electrons/anti-electrons is effectively limited to 2-dimensional curved rim of the wormhole, for equally charged particles [emitting equal photon species], the accumulation of emitted photon species would result in repulsive force (expansion of the wormhole [length] due to increased temperature, decreasing cross-section).

However, if emitted photon species are spin anti-aligned, even here the attractive force may be created due to boson condensation, and if magnetic field lines [pairings] are stable long enough will result in bosonic coupling (fusion) of interacting particles - probability for this being inversely proportional to 
temperature/density.

Note that emitted photon species may be in the form of quanta of orbital momentum (having the radius of the wormhole cross-section) or spin momentum (collapsed orbital momentum) with orbital radius equal to the wormhole cross-section radius.

Note also that in both types of interaction (attractive/repulsive), sum of radii of interacting particles must remain relatively constant - this is ensured through changes in other entanglements.

\subsection{Primary atom radius}

Generally, radius of the atom is equal to the radius of its outermost electron orbit.

Primary radius of the atom is equal to the orbital radius of its outermost primary component.

At minimum, it is equal to the general (outermost electron orbit) radius of the atom. However, at equilibrium - with all primary neutrinos present, it may be over twice that radius.

\subsection{MAU}

MAU or Mars relative Astronomical Unit is a unit of distance, relative to the orbit of the outermost positive charge in an atom. $1 \mathrm{MAU}$ is equal to the distance of such charge from the nucleus.

On $U_{1}$ scale ${ }^{10} \mathrm{C}$ atom, $1 \mathrm{MAU}$ is equal to the distance of Mars from the Sun.

\subsection{Weak nuclear decay}

Weak nuclear decay transforms a neutron into a proton or vice versa. If these are parts of an atom, this is nuclear transmutation - transformation of one atom of an element into an atom of another element.

With scale invariance of gravitational fields, neutrinos and anti-neutrinos can be, like electrons, bound to atomic nuclei (and, as other fermions, grouped into pairs). In equilibrium, the number of bound electron (e) neutrinos and electron anti-neutrinos within the [primary] radius of the atom correspond to the number of protons and neutrons, respectively. These are, together with the nuclei and electrons, the primary components of the atom.

Decay process involves annihilation of neutrinos and anti-neutrinos. 


\subsection{1. $\beta^{-}$decay}

Transformation of a neutron to a proton, with the emission of excess energy:

$$
n \rightarrow p^{+}+\Delta E
$$

Here, bound non-primary e neutrino and bound primary e anti-neutrino annihilate to produce, depending on energy, either an electron/positron $\left(\mathrm{e}^{-} / \mathrm{e}^{+}\right)$pair, or up/anti-up quark pair:

$$
e_{v}+\bar{v}_{e} \rightarrow\left(e^{-}+e^{+}\right) \|\left(u^{+}+u^{-}\right)
$$

In case of electron/positron production, positron further partially annihilates with the down quark (here, both are composite particles), producing neutrino/anti-neutrino pair and up quark:

$$
e^{+}+d^{-} \rightarrow u^{+}+v_{e}+\bar{v}_{e}
$$

Neutrino bounds to the atom [as a primary component], while anti-neutrino and electron are ejected in a spin paired state (boson), before separating again:

$$
e^{-}+\bar{v}_{e} \rightarrow W^{-} \rightarrow e^{-}+\bar{v}_{e}
$$

In case of up/anti-up quark production in the first step, the up quark is absorbed, while anti-up quark pairs with the down quark before ejection:

$$
u^{-}+d^{-} \rightarrow W^{-} \rightarrow u^{-}+d^{-}
$$

Note that a decay of $\mathbf{W}^{-}$into an electron and anti-neutrino even when it is created from anti-up and down quarks would suggest that charge in electron is a composite of $1 / 3$ and $2 / 3$ charge quanta. In the decay of a proton to neutron through electron capture, electron could then [inverse] decay to $u^{-}$and $d^{-}$by pairing with an anti-neutrino (inflating to $\mathbf{W}^{-}$boson), $\mathrm{u}^{-}$would annihilate with $\mathrm{u}^{+}$, leaving 2 down and 1 up quark, forming a neutron.

Outside of atom, the pairing is unstable (short-lived), except at extreme conditions.

Note that, in this case, to conserve equilibrium conditions, one of bound non-primary e neutrinos must reduce its orbit to become a primary component.

$\beta^{-}$decay is the effective transformation of a down quark to up quark of the atom nucleus.

Note that a $\mathbf{W}$ boson has a rest mass over $\mathbf{8 0}$ times that of a neutron and orders of magnitude more than that of down and up quarks. 
Thus, the production of a $\mathbf{W}$ boson is apparently a violation of energy conservation. In QM this is solved with time-energy uncertainty principle which allows production of such particles out of vacuum providing they decay quickly (lifetime of a $\mathbf{W}$ boson is $\sim 10^{-25}$ seconds).

However, mass of the boson is also considered variable with probability of deviation from rest mass decreasing fast with the amount of deviation, thus, making the probability of beta decay proportional to creation of a low mass $\mathbf{W}$ boson.

In reality, there is no violation of energy conservation and high mass of a $\mathbf{W}$ boson is actually the result of conservation of energy due to momentum - energy equivalence (note that, per CR postulates, even rest mass has a momentum), where one component of the angular momentum is exchanged for the other. In this case, the angular momentum of a particle orbiting the nucleus is collapsed [localized] to a spin momentum, where radius has been exchanged for mass.

This is, generally, the process of conversion of a polarized component of the general force into a neutral (gravitational) component - effectively, the exchange of charge for mass.

If this is temporary, like in case of $\boldsymbol{\beta}$ decay, radius is inflated again (restoring em component) and two components of the force are again separated (concentrated) into multiple particles (although neither can be absolutely zero for any particle).

Thus, although $\mathbf{W}$ boson is charged, and charge is conserved between the initial and final state of the system, it is not conserved in the boson itself (unless created mass is indeed extremely low compared to rest mass) - otherwise the conservation of energy would be violated.

It will be shown later that, in reality, time-energy uncertainty indeed manifests itself in the exchange of angular momentum components.

\subsection{2. $\beta^{+}$decay}

Transformation of a proton to a neutron, with the emission of excess energy:

$$
p^{+} \rightarrow n+\Delta E
$$

Here, bound primary e neutrino and bound non-primary e anti-neutrino annihilate to produce either an electron/positron $\left(\mathrm{e}^{-} / \mathrm{e}^{+}\right)$pair, or down/anti-down quark pair:

$$
e_{v}+\bar{v}_{e} \rightarrow\left(e^{-}+e^{+}\right) \|\left(d^{+}+d^{-}\right)
$$

In case of electron/positron production, electron further partially annihilates with the up quark (here, both are composite particles), producing neutrino/anti-neutrino pair and a down quark: 


$$
e^{-}+u^{+} \rightarrow d^{-}+v_{e}+\bar{v}_{e}
$$

The anti-neutrino bounds to the atom [as a primary component], while neutrino and positron are ejected in a spin paired state (boson), before separating again:

$$
e^{+}+v_{e} \rightarrow W^{+} \rightarrow e^{+}+v_{e}
$$

In case of down/anti-down quark production in the first step, the down quark is absorbed, while anti-down quark pairs with the up quark before ejection:

$$
u^{+}+d^{+} \rightarrow W^{+} \rightarrow u^{+}+d^{+}
$$

Note that, in this case, to conserve equilibrium conditions, one of bound non-primary e anti-neutrinos must reduce its orbit to become a primary component.

$\beta^{+}$decay is the effective transformation of an up quark to down quark of the atom nucleus.

\subsubsection{Inverse $\beta$ decay}

Transformation of a proton to a neutron by electron anti-neutrino scattering. Generally, this interaction will occur when the atom is not in equilibrium, more specifically - the number of bound e neutrinos is lower than the number of protons.

$$
\bar{v}_{e}+p^{+} \rightarrow e^{+}+n
$$

In this process, e anti-neutrino annihilates with a bound non-primary e neutrino, initiating a $\beta^{+}$decay with electron/positron product:

$$
\begin{gathered}
e_{v}+\bar{v}_{e} \rightarrow e^{-}+e^{+} \\
e^{-}+u^{+} \rightarrow d^{-}+v_{e}+\bar{v}_{e}
\end{gathered}
$$

However, since the number of bound primary e neutrinos was initially lower than the number of protons, now even the created neutrino is bound (as a non-primary component) rather than ejected with a positron:

$$
e^{+} \rightarrow e^{+}
$$

\subsubsection{Electron capture}

Transformation of a proton to a neutron by electron capture.

$$
p^{+}+e^{-} \rightarrow v_{e}+n
$$

Bound electrons induce the creation of positrons from the atom nucleus, filling its outer energy levels. In low energy conditions this may not be possible and one of the innermost electrons may be captured to fill the 
vacant level. However, the electron in this level is highly unstable, it is attracted to the outer proton core where it partially annihilates with the up quark, proceeding further as $\beta^{+}$decay:

$$
e^{-}+u^{+} \rightarrow d^{-}+v_{e}+\bar{v}_{e}
$$

The anti-neutrino bounds to the atom as a primary component, while neutrino gets ejected. Like in case of inverse $\beta$ decay, there is no $\mathbf{W}$ boson creation as no positrons were created:

$$
v_{e} \rightarrow v_{e}
$$




\section{Initial structure hypothesis}

In planetary systems, outer (gas) planets are [groups of] electrons, while inner (terrestrial) planets are [groups of] positrons whose gravitational maximums have been extracted from the system nucleus to balance the electrons.

A planet can be in $1 e$ or $2 e$ configuration, while the star is the superposition of nuclei partons (quarks). Inner and outer dwarf planets in a planetary system are bound anti-neutrinos and neutrinos, respectively.

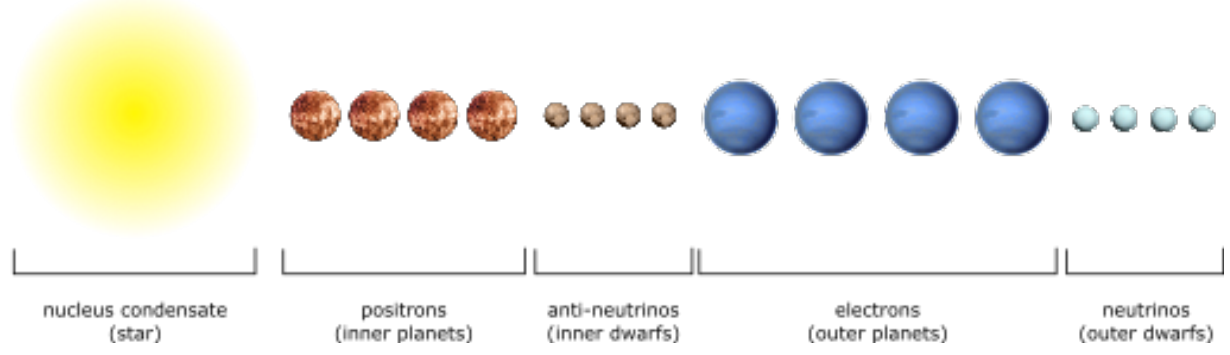

Fig. 1: Primary components of the Solar System (planet images source: Pixabay/OpenClipart-Vectors ${ }^{3}$ ) Primary components of the Solar System are shown on Fig. 1.

Note that components of momentum are exchangeable and it is the reason why bound neutrinos/antineutrinos have significantly inflated real mass compared to free neutrinos/anti-neutrinos.

The current Solar System is in a ${ }^{10} \mathrm{C}$ atom configuration, in transition to ${ }^{10} \mathrm{Be}$ through $\beta^{+}$decay.

a)

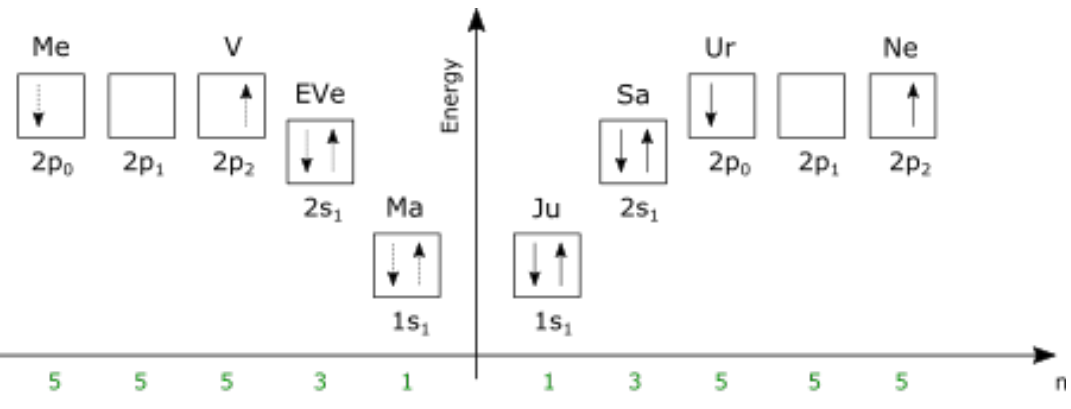

b)

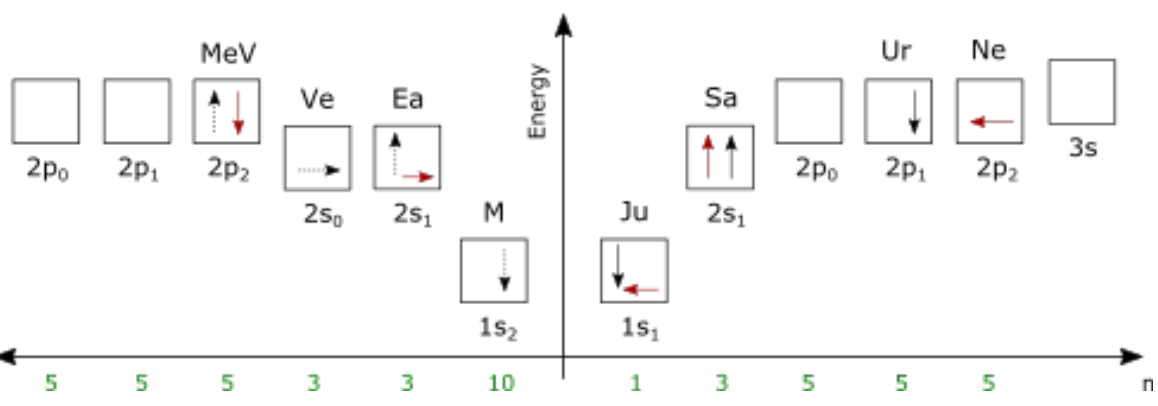


Fig. 2: a) stable ${ }^{12} \mathrm{C}$ energy levels b) current Solar System $\left({ }^{10} \mathrm{C}\right)$ energy levels

Fig. 2 a) shows the configuration of ${ }^{12} \mathrm{C}$ atom (stable on standard scale, unstable on $U_{1}$ ), on the left is the configuration of positrons, on the right is the configuration of electrons.

Fig. 2 b) shows the possible configuration of ${ }^{10} \mathrm{C}$ atom (unstable on standard scale room temperature, stable on $\mathrm{U}_{\mathbf{1}}$ scale).

Note that the splitting of $\mathbf{s}$ levels on the left side should be attributed to the lack of neutrons, as they provide the neutral gravitational energy to inner planets.

This generally does not happen on the right side where this energy is provided by protons.

Note also that, due to condensation (the system may be carbon-like, not carbon), principal quantum number has an imaginary value (n) and effective value $(\mathbf{N})$ which here is either $\mathbf{1}$ or $\mathbf{2}$.

Note also that 2 particles are allowed per sub-shell and there is no reason for a lone electron not to pair up with a bound neutrino, forming a [W] boson, although such pairing may be extremely unstable at room temperature/density, oscillating in existence.

\subsection{General deduction of quantum structure}

Here is an example how the element and exact isotope species can be determined from the number and types of planets.

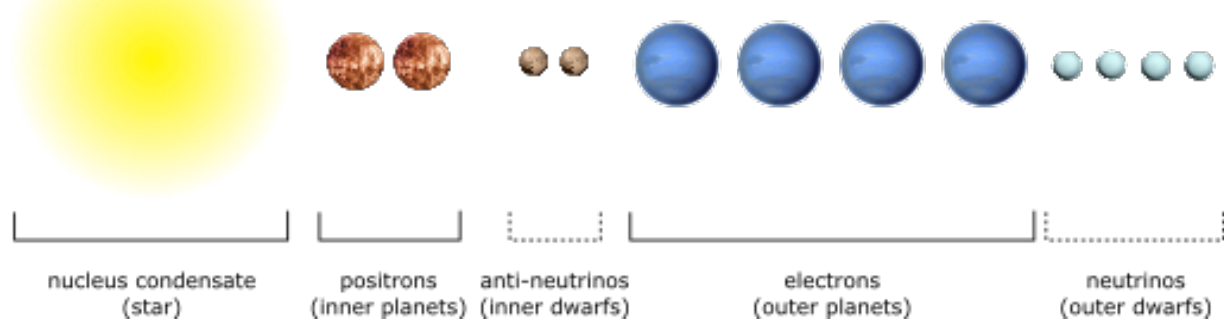

Fig. 1: Primary components of the TOI-178 System (planet images source: Pixabay/OpenClipart-Vectors ${ }^{4}$ )

The discovered (star, planets) and hypothesized (dwarf planets) components of TOI-178 system are shown on Fig. 1.

With the assumption of maximum 2 electrons (positrons) per planet, the TOI-178 system has these 
restrictions on the number of particles:

- 2 terrestrial planets limit the number of positrons to $\mathbf{2}-\mathbf{4}$,

- 4 gas planets limit the number of electrons to $\mathbf{4}$ - $\mathbf{8}$.

Since the intersection of the two groups contains only one solution (4), the TOI-178 system must be a Beryllium atom.

If the number of terrestrial planets corresponds to number of neutrons, this must be ${ }^{6}{ }^{6} \mathrm{Be}$ isotope.

This can be confirmed by comparing the mass of the TOI-178 system [star] with the mass of the Sun. Assuming that the Solar System is ${ }^{10} \mathrm{C}$ (or ${ }^{10} \mathrm{Be}$ ), the determined mass of TOI-178 $\left(0.647^{+0.035 /-0.032} \mathbf{M}_{\odot}{ }^{5}\right.$ ) agrees well with the hypothesis.

However, the measured mass is still somewhat larger than expected - reasons for this will be discussed later.

Note that it is also possible for the number of terrestrial planets to actually reduce with the increasing number of neutrons due to increased gravitational potential provided by neutrons, but this also requires either low [properly scaled] temperatures for boson condensation of charges beyond the $2 e$ configuration or excessive number of neutrons compared to protons.

Note also that, in heavy elements, due to condensation of mass and with no significant change in atomic radii, there is a possibility for all planets of a system to be gaseous giants. However, equivalents of dwarf planets should exist in between positively and negatively charged giants - in that case, these should be of significantly lower mass and may be equivalents of terrestrial planets with no significant magnetic dipoles.

The number of bound [primary] anti-neutrinos should also correspond to number of neutrons, while the number of bound [primary] neutrinos should correspond to the number of protons.

Note that, while bound anti-neutrinos/neutrinos should correspond to the number of neutrons/protons, they will not necessarily be in the same configuration as positrons/electrons.

Thus, it is possible that TOI-178 has a single inner dwarf planet (holding 2 anti-neutrinos) instead of two, and two outer primary dwarf planets instead of four.

Additional particles may also be bound to the system, however, the orbits of these should lie beyond 
the primary components, unless these are lower mass particles with no distinct gravitational maximum (asteroids, comets).

Note also that, with the exception of the innermost planet, planets of the TOI-178 are in orbital resonance (18:9:6:4:3). The pattern does suggest one additional particle (or a binary) between the terrestrial and gas planets, one that would complete 13 revolutions for every 18 revolutions of the second planet (pattern 18:13:9:6:4:3). 


\section{Quantum nature}

Solar System is a Carbon-10 atom in the current state. Due to extreme conditions some of its components are at the lowest energy level - multiple nucleons have condensed into a single nucleus, orbitals are two dimensional (collapsed from spherical cloud structure), highly aligned (same plane), and momentum carriers are (scaled) point like structures.

Scale invariance of physical laws requires that non-dimensional ratios - those of radii, masses and velocities (energies in general) in two systems of the same species (carbon in this case) but different scale are equal.

Radius of the outermost electron of ${ }^{10} \mathrm{C}$ can be obtained from Neptune spin and orbital radius:

$$
\frac{\text { Neptune spin radius }}{\text { Neptune orbital radius }}=\frac{10 \mathrm{C} \text { outermost electron spin radius }}{10 \mathrm{C} \text { outermost electron orbital radius }}=\frac{R_{U_{1}}}{r_{U_{1}}}=\frac{R_{U_{0}}}{r_{U_{0}}}
$$

This gives electron radius $R_{U_{0}}=\mathbf{3 . 8 3 4 2 9 8 0 9 6} * 10^{-16} \mathrm{~m}$. Note that radii of particles inside the atom can be different than outside of atom.

Generally, radii are affected by kinetic energy and oscillate with mass.

Sun core radius from ${ }^{10} \mathrm{C}$ nucleus radius and outermost electron radius:

$$
\frac{10 \mathrm{C} \text { nucleus charge radius }}{10 \mathrm{C} \text { outermost electron spin radius }}=\frac{\text { Sun core radius }}{\text { Neptune spin radius }}
$$

The above gives Sun core radius of $173894.6069 \mathrm{~km}$, or $1 / 4$ of the apparent Sun radius, in agreement with experimentally obtained values of Sun core size. More precisely, this is the Sun outer core [discontinuity] radius and also [approximately] $\mathbf{U}_{\mathbf{1}}$ classical electron radius.

Proton radius approximation:

$$
\frac{\text { Sun radius }}{\text { Solar System charge radius }}=\frac{P}{N} \frac{10 * \text { proton radius }}{\text { Carbon-10 charge radius }}
$$

The factor $\mathrm{P} / \mathrm{N}=\mathbf{6 / 4}=\mathbf{3 / 2}$ is the ratio of protons to neutrons in Carbon-10 atom, factor 10 is the number of nucleons $(\mathrm{P}+\mathrm{N})$.

The above gives $0.7222958833 * 10^{-15} \mathrm{~m}=0.7222958833 \mathrm{fm}$ for the proton radius, close to experimentally obtained value of $\mathbf{0 . 8 4 1 4}(\mathbf{1 9}) \mathrm{fm}\left(2018 \mathrm{CODATA}^{6}\right)$.

Same result can be obtained by using spin radii: 


$$
\frac{\text { Sun radius }}{\text { Neptune spin radius }}=\frac{P}{N} \frac{10 * \text { proton radius }}{10 \mathrm{C} \text { outermost electron spin radius }}
$$

A precise value can be obtained by taking into account the influence of quarks instead of P/N (this will be elaborated later):

$$
\frac{\text { Sun radius }}{\text { Solar System charge radius }}\left[\left(\frac{2}{3}\right)^{2}+\frac{1}{3}\right]=\frac{10^{*} \text { proton radius }}{\text { Carbon-10 charge radius }}
$$

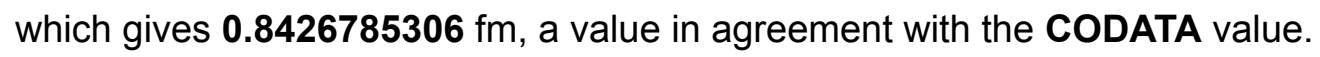

The radius of the proton is not constant, it is expected to be shrinking as the Solar System expands during the weak evolution of the current state $(6 p 4 n)$.

Comparing masses:

$$
\frac{\text { Sun mass }}{\text { Neptune mass }} \approx \frac{10 \mathrm{C} \text { nucleus mass }}{10 \mathrm{C} \text { outermost electron mass }}
$$

This gives:

$$
19416.48033 \approx 18260.0087
$$

The above shows mass ratios agree not only to the order of magnitude but are actually very close in value. The excess energy is:

$$
\Delta M=\text { Sun mass }-\frac{10 \mathrm{C} \text { nucleus mass }}{10 \mathrm{C} \text { outermost electron mass }} \text { Neptune mass }=1.18437729 * 10^{29} \mathrm{~kg} \approx 6 \% \text { Sun mass }
$$

and it must be the kinetic energy of the Solar System (discrepancy arises due to non-invariant reference frames in the mass measurement - the mass of a standard ${ }^{10} \mathrm{C}$ atom is measured from an external frame, while the mass of the Solar System is derived from within that system and improperly treated as rest mass).

Although the Solar System is at rest relative to us, kinetic energy of the system due to interaction with the intergalactic $\left(\mathbf{U}_{\mathbf{1}}\right)$ medium must be stored somewhere in the system. This capacitor is the gravitational field (imaginary mass).

Note that this implies non-isotropic storage of kinetic energy as gravitational potential, such that it is mostly concentrated in the Sun - density distribution following the gravitational law $\left(\sim 1 / r^{2}\right)$.

From this one can calculate the scaled speed of light for the $\mathbf{U}_{\mathbf{1}}$ scale $\left(\mathbf{c}_{\mathbf{1}}\right)$ :

$$
M=M_{\odot}-\Delta M=1.870062271 * 10^{30} \mathrm{~kg}
$$




$$
\begin{gathered}
v=v_{s}+v_{p} \\
M_{\odot}=\frac{M}{\sqrt{1-\frac{v^{2}}{c_{1}^{2}}}} \\
c_{1}=\frac{v}{\sqrt{1-\frac{M^{2}}{M_{\odot}^{2}}}}
\end{gathered}
$$

where $\mathbf{v}$ is the cumulative velocity against the CMB (Constant Microwave Background) radiation, a sum of secondary velocity $\mathbf{v}_{\mathbf{s}}$ (velocity of the Solar System against $\mathbf{C M B}$ ) and primary velocity $\mathbf{v}_{\mathbf{p}}$ (velocity of the local galactic group against $\mathbf{C M B}$ ).

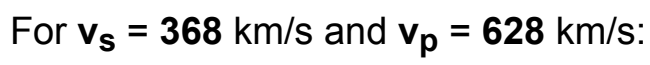

$$
c_{1}=2.93 * 10^{6} \mathrm{~m} / \mathrm{s}
$$

Obtained $\mathbf{c}_{\mathbf{1}}$ will be confirmed later in a different calculation.

Comparing masses of systems of different scales requires proper relativistic treatment. Apart from the speed of light being different between the scales, a proper reference frame must be chosen. In case of comparison of $\mathbf{U}_{\mathbf{1}}$ scale system (such as Solar System) with a $\mathbf{U}_{\mathbf{0}}$ system (such as ${ }^{10} \mathbf{C}$ atom) a proper reference frame is the CMB (Constant Microwave Background) radiation rest frame.

Proper equation is thus (for $\left.\mathbf{v}_{\mathbf{1}}=\mathbf{v}_{\mathbf{0}}=\mathbf{v}\right)$ :

$$
\frac{\text { Sun mass }}{\text { Neptune mass }} \sqrt{1-\frac{v^{2}}{c_{1}^{2}}}=\frac{10 \mathrm{C} \text { nucleus mass }}{10 \mathrm{C} \text { outermost electron mass }} \sqrt{1-\frac{v^{2}}{c_{0}^{2}}}
$$

$$
\begin{array}{r}
v=v_{\odot}=\text { speed relative to CMB }=996 \mathrm{~km} / \mathrm{s} \\
c_{1}=\text { speed of light on } U_{1} \text { scale }=2.93 * 10^{6} \mathrm{~m} / \mathrm{s} \\
c_{0}=c=\text { speed of light on } U_{0} \text { scale }=2.99792458 * 10^{8} \mathrm{~m} / \mathrm{s}
\end{array}
$$

Note that $\mathrm{CMB}$ radiation is of $U_{-1}$ scale.

Note also that maximum speed $\left(\mathbf{c}_{\mathbf{n}}\right)$ depends on pressure and density of space and it is generally not equal to the standard speed of light. Here thus, even though the term speed of light may be used, $\mathbf{c}_{\mathbf{1}}$ should be understood as maximum speed of $\mathbf{U}_{\mathbf{1}}$ particles in flat intergalactic space equivalent to standard $\left(\mathbf{U}_{\mathbf{0}}\right)$ vacuum.

Within the galaxy, speed limit for orbiting bodies is generally defined by the gravitational maximum 
(event horizon) of the well - stars do exist orbiting black holes faster than $\mathbf{c}_{\mathbf{1}}$, however, probability for stars moving at speeds $\geq \mathbf{c}_{\mathbf{1}}$ outside of galaxies is negligible.

One can now attempt to resolve the excess mass of TOI-178 $\left({ }^{6} \mathrm{Be}\right)$ system. Assuming its velocity [relative to CMB] is $\mathbf{7 7 . 2 2} \mathrm{km} / \mathrm{s}$ larger than Sun's velocity, its mass should be:

$$
M_{T O I-178}=\frac{M_{B e-6}}{M_{C-10}} M \frac{1}{\sqrt{1-\frac{v^{2}}{c_{1}^{2}}}}=1.207764563 * 10^{30} \mathrm{~kg}=0.607 M_{\odot}=0.646 \mathrm{M}
$$

$$
\begin{array}{r}
M_{\mathrm{Be}-6}=\text { rest mass of the }{ }^{6} \mathrm{Be} \text { atom }=6.0197 \mathrm{u} \\
M_{\mathrm{C}-10}=\text { rest mass of the }{ }^{10} \mathrm{C} \text { atom }=10.016853 \mathrm{u} \\
M=\text { rest mass of the Sun (relative to } \mathrm{CMB} \text { ) }=1.870062271 * 10^{30} \mathrm{~kg} \\
\mathrm{v}=1073.22 \mathrm{~km} / \mathrm{s}
\end{array}
$$

However, mass of TOI-178 obtained from measurements is $0.650^{+0.027 /-0.029} \mathbf{M}_{\odot}{ }^{7}$.

Apparently, measured mass is bigger by the relativistic [omega] factor:

$$
\frac{1}{\sqrt{1-\frac{v^{2}}{c_{1}^{2}}}} \approx \frac{v}{v_{\odot}}
$$

The cause of discrepancy is, again, in the reference frame - calculation is done relative to CMB, while measurements were done from the Solar System (Earth) reference frame.

From such reference frame Sun is at rest and its rest mass is equal to relativistic mass relative to CMB, $M_{\odot}\left(1.988500 * 10^{30} \mathrm{~kg}\right)$.

However, one must take into account the radial velocity [relative to the Sun] of TOI-178. Relative to the Solar System, the mass of TOI-178 should thus be:

$$
\begin{aligned}
& M_{T O I-178}=\frac{M_{B e-6}}{M_{C-10}} \frac{M}{\sqrt{1-\frac{\left(v_{\odot}+v_{r}\right)^{2}}{c_{1}{ }^{2}}}} \frac{1}{\sqrt{1-\frac{v^{2}}{c_{1}^{2}}}} \\
& \mathrm{~V}_{\mathrm{r}}=\text { radial velocity of TOI-178 }=\mathbf{5 7 . 4} \pm \mathbf{0 . 5} \mathrm{km} / \mathrm{s}
\end{aligned}
$$

This gives $\mathbf{0 . 6 5 0} \mathbf{M}_{\odot}$ for the mass of TOI-178, in agreement with measurements.

Note that relativistic effects must be physical, implying the existence of a lensing effect proportional to kinetic energy relative to $\mathrm{CMB}$. 
Solar System is thus a [negatively] polarized reference frame relative to TOI-178 and to convert the measurement to a proper [neutral] reference frame, one must multiply the measured value with a positively polarized omega factor:

$$
\left(\frac{1}{\sqrt{1-\frac{\left(v_{\odot}+v_{r}\right)^{2}}{c_{1}{ }^{2}}}}\right)^{-1}=\sqrt{1-\frac{\left(v_{\odot}+v_{r}\right)^{2}}{c_{1}{ }^{2}}}
$$

If the Carbon atom is the Solar System, Carbon photon is the Carbon atom of lower scale (vertical energy level).

One can thus calculate the [average] mass of photons, ie. electron photon:

$$
\begin{gathered}
\frac{\text { Neptune mass }}{10 \mathrm{C} \text { outermost electron mass }}=\frac{10 \mathrm{C} \text { outermost electron mass }}{\text { e photon mass }} \\
\text { e photon mass }=\frac{(10 \mathrm{C} \text { outermost electron mass })^{2}}{\text { Neptune mass }}=8.102214736 * 10^{-87} \mathrm{~kg}
\end{gathered}
$$

However, obtained photon mass above assumes linear progression of discrete states of scale invariance (vertical symmetry, distance in scale from $\mathbf{U}_{0}$ to both $\mathbf{U}_{1}$ and $\mathbf{U}_{-1}$ is equal), which is against the postulates of $\mathbf{C R}$ - although this can be the mass of the photon in another time (another cycle state).

There can be no symmetry between current space and time, but due to cyclic nature of a universe and with cycle states being inverse of each other, symmetry would exist between past and future dimensions (space and time dimensions exchange in a way that current space is symmetric with previous space).

Thus, CR predicts asymmetric invariance with exponential progression of discrete vertical states. Using this prediction, the masses of standard photon [scale] electron equivalent (half-photon) and carbon graviton neutrino have been calculated already in CR (yielding $9.10938356 * 10^{-73} \mathrm{~kg}$ for the photon e mass, 1.663337576 * $10^{-68} \mathrm{~kg}$ for the half-neutrino mass), but the values can also be obtained using EH operator.

Using EH factor 6/4 on the orders of magnitude of mass distances:

$$
\log _{10}\left(\frac{M_{U_{1}}}{M_{e}}\right)=E H_{6 / 4}\left[\log _{10}\left(\frac{M_{e}}{M_{n}}\right), \log _{10}\left(\frac{m_{U_{1}}}{M_{n}}\right)\right]
$$

gives $M_{n}=3.910613743 * 10^{-68} \mathrm{~kg}$ for the mass of neutrino in current cycle state, and $m_{U_{1}}=6.06011796$ * $10^{19} \mathrm{~kg}$ for the initial real mass of Neptune in current cycle state. 
Here, $\mathbf{M}_{\mathrm{p}}=\mathbf{M}_{\mathrm{n}} / \mathrm{m}_{\mathbf{U}_{1}}=\mathbf{6 . 4 5 3 0 3 2 3 8 3}{ }^{*} \mathbf{1 0}^{-88} \mathrm{~kg}$ could be interpreted as the mass of carbon photon in inverse cycle state.

Mass of current photon can now be obtained from $\mathbf{M n}$ :

$$
M_{p}=\frac{M_{e}}{{ }^{10} C \text { atom mass }} M_{n} \approx M_{n} * 10^{-5}
$$

Note that, in the current state the ratio of magnitude distances from electron to neutrino and from electron to $U_{1}$ electron (Neptune) is:

$$
\log _{10}\left(\frac{M_{e}}{M_{n}}\right)\left[\log _{10}\left(\frac{M_{U_{1}}}{M_{e}}\right)\right]^{-1}=\frac{4}{6} \frac{5}{5}=\frac{2}{3}
$$

So, for the inverse state $(4 p 6 n)$ :

$$
\begin{gathered}
\log _{10}\left(\frac{M_{e}}{M_{n}}\right)\left[\log _{10}\left(\frac{M_{U_{1}}}{M_{e}}\right)\right]^{-1}=\frac{6}{4} \frac{3}{7}=\frac{9}{14} \\
\log _{10}\left(\frac{M_{U_{1}}}{M_{e}}\right)=E H_{4 / 6}\left[\log _{10}\left(\frac{M_{e}}{M_{n}}\right), \log _{10}\left(\frac{m_{U_{1}}}{M_{n}}\right)\right]
\end{gathered}
$$

Respecting conditions for the $\mathbf{E H}$ inverse, the following values are obtained: mass $\mathbf{M}_{\mathrm{e}}=\mathbf{3 . 9 1 0 6 1 3 7 4 3}$ * $10^{-68} \mathrm{~kg}$ of $\left[{ }^{10} \mathrm{C}\right.$ outermost] electron [equivalent] in $\mathbf{U}_{-1} .4 \mathrm{p} 6 \mathrm{n}\left(=\mathbf{M}_{\mathrm{n}}\right.$ in $\left.\mathbf{U}_{0} .6 \mathrm{p} 4 \mathrm{n}\right), \mathbf{M}_{\mathbf{U}_{1}}=\mathbf{9 . 1 0 9 3 8 3 5 6}^{*} \mathbf{1 0}^{-31} \mathrm{~kg}$ for the imaginary mass of Neptune in $U_{-1} .4 p 6 n\left(=M_{e}\right.$ in $\left.U_{0} .6 p 4 n\right), M_{n}=3.719162593 * 10^{-92} \mathrm{~kg}$ for the mass of neutrino in $U_{-1} .4 p 6 n, m_{U_{1}}=4.18129939 * 10^{-36} \mathrm{~kg}$ for the real mass of Neptune in $U_{-1} .4 p 6 n\left(=m_{e}\right.$ in $\left.U_{0} .6 p 4 n\right)$.

Note that here, mass of photon is obtained from:

$$
M_{p}=\frac{{ }^{10} C \text { atom mass }}{M_{e}} M_{n}=6.791044478 * 10^{-88} \mathrm{~kg}
$$

suggesting inverted roles of photon and neutrino.

Using conservation of angular momentum between the ${ }^{10} \mathrm{C}$ atom and the Solar System (Solar System = vertical energy state of the ${ }^{10} \mathrm{C}$ atom), one may attempt to calculate the angular velocity of the outermost 
electron in ${ }^{10} \mathrm{C}$ atom:

$$
\begin{gathered}
L=m v r=\frac{v}{r} m r^{2} \\
M_{U_{1}} v_{U_{1}} r_{U_{1}}=M_{U_{0}} v_{U_{0}} r_{U_{0}} \\
v_{U_{0}}=\frac{M_{U_{1}} v_{U_{1}} r_{U_{1}}}{M_{U_{0}} r_{U_{0}}}=3.920242676 * 10^{82} \frac{\mathrm{m}}{\mathrm{s}}
\end{gathered}
$$

The above gives the outermost electron velocity in the case of conversion of both mass and orbital radius into angular velocity, for a point energy in constant vacuum density.

However, mass $\mathbf{M}_{\mathbf{U}_{0}}$ must have been relativistic before the speed limit was reached (vertical energy level changed) and it became the rest mass $\mathbf{M}_{\mathbf{U}_{1}}$.

Thus, in order to get the orbital velocity just before the [vertical] energy level change, rest mass on one scale must be equalized with relativistic mass on another $\left(\mathbf{M}_{\mathbf{U}_{1}}=\mathbf{M}_{\mathbf{U}_{0}}\right)$ :

$$
v_{U_{0}}=\frac{v_{U_{1}} r_{U_{1}}}{r_{U_{0}}}=3.486882257 * 10^{26} \frac{\mathrm{m}}{\mathrm{s}}
$$

With real mass not participating in inflation (maximums inflate naked), this velocity is the velocity of space, making it valid even in the context of General Relativity (GR).

Using conservation of energy, one can now obtain the velocity of the outermost electron in standard nonexcited ${ }^{10} \mathrm{C}$ atom:

$$
\begin{gathered}
E_{-1}=E_{0} \\
\rho_{v a c} * V_{U_{0}} * v_{U_{0}}{ }^{2}=M_{U_{0}} * v^{2} \\
\rho_{v a c}=\text { mean vacuum energy density }=9.9 * 10^{-27} \frac{\mathrm{kg}}{\mathrm{m}^{3}} \\
\rho_{v a c} * \frac{4}{3} \pi\left(R_{U_{0}}\right)^{3} * v_{U_{0}}{ }^{2}=M_{U_{0}} * v^{2} \\
2.842208873 * 10^{-19}=M_{U_{0}} * v^{2}
\end{gathered}
$$

This gives $\mathrm{v}=\mathbf{5 . 5 8 5 8 3 7 3 5 6}{ }^{*} \mathbf{1 0}^{\mathbf{5}} \mathrm{m} / \mathrm{s}$, for the velocity of the outermost electron of a standard ${ }^{10} \mathrm{C}$ atom [in Solar System equivalent state].

Note that the product of density and volume on the left should be the mass of a standard photon $\left(2.337660431 * 10^{-72} \mathrm{~kg}\right)$, and it is indeed roughly equal to previously calculated photon rest mass.

To confirm validity of the result one can calculate this velocity differently. Introducing the term total velocity $\left(\mathbf{v}_{\text {tot }}\right)$ as the sum of electron's spin and angular velocity. 
Per CR postulates, every spin momentum must be an orbital momentum. If one assumes that, once captured by the atom, the outermost electron self-orbital (spin) momentum becomes the nucleus-orbital momentum, in ground state (with quantum number $\mathbf{I}=\mathbf{0}$ ) thus, total momentum of the electron is:

$$
\begin{gathered}
m r^{2} \omega_{t o t}=\frac{1}{2} \hbar \\
v_{t o t}=r \omega_{t o t}=\frac{1}{2} \frac{\hbar}{m r}
\end{gathered}
$$

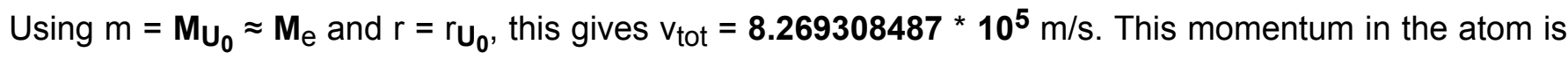
further divided between orbital and spin momentum. With the ratio of velocities equal to Neptune spin/orbital velocity, one obtains electron orbital velocity:

$$
v=v_{U_{0}}=\frac{v_{t o t}}{1+\frac{s_{U_{1}}}{v_{U_{1}}}}=5.5550351679 * 10^{5} \frac{\mathrm{m}}{\mathrm{s}}
$$

The result is obtained from the following:

$$
\begin{gathered}
v_{t o t}=v_{a}+v_{s} \\
M_{e} v_{t o t} r_{a}=\frac{1}{2} \hbar
\end{gathered}
$$

Splitting the momentum in scalar space:

$$
\begin{gathered}
m_{r e} v_{a} r_{a}+m_{i m g} v_{s} r_{s}=M_{e} v_{t o t} r_{a} \\
\frac{m_{r e}}{M_{e}} v_{a}+\frac{m_{i m g}}{M_{e}} v_{s} \frac{r_{s}}{r_{a}}=v_{t o t}
\end{gathered}
$$

and assuming:

$$
m_{r e}=M_{e}
$$

from Q1.2 and Q1.4, follows:

$$
\begin{array}{r}
m_{i m g}=M_{e} \frac{r_{a}}{r_{s}} \quad(\mathrm{Q} 1.5) \\
\mathbf{M}_{\mathrm{e}}=\text { standard electron mass }=9.10938356 * 10^{-31} \mathrm{~kg} \\
\mathrm{r}_{\mathrm{a}}=\mathrm{r}_{\mathrm{U}_{0}}=\text { orbital radius of the outermost }{ }^{10} \mathrm{C} \text { electron }=70 * 10^{-12} \mathrm{~m} \\
\mathrm{r}_{\mathrm{s}}=\mathbf{R}_{\mathrm{U}_{0}}=\text { spin radius of the outermost }{ }^{10} \mathrm{C} \text { electron }=3.834298096 * 10^{-16} \mathrm{~m}
\end{array}
$$

In order for Q1.2 to be satisfied, masses of orbital and spin momenta must be different. With orbital 
mass equal to standard electron mass, spin mass $\mathbf{m}_{\text {img }}$ is:

$$
\begin{gathered}
m_{i m g}=1.66303410 * 10^{-25} \mathrm{~kg}=9.99817551 *{ }^{10} \mathrm{C} \text { nucleus mass } \\
m_{i m g} \approx 10 *{ }^{10} \mathrm{C} \text { nucleus mass } \approx 93.3 \mathrm{GeV} / \mathrm{c}^{2}
\end{gathered}
$$

Note that the increase in electron spin mass $\mathbf{m}_{\mathbf{i m g}}$ is proportional to the increase of nucleus mass. In both, mass component of the spin momentum was increased at the expense of other components, as with electro-magnetic coupling the em energy was converting to neutral gravitational energy.

Note also that, from this, it is possible to derive the rest mass and rest charge radii of a free electron. Assuming radius inflation proportional to mass inflation, rest mass radius of a free electron is:

$$
r_{s_{e}}=\frac{r_{s}}{r_{a}} r_{s}=\frac{r_{s}^{2}}{r_{a}}=2.100 * 10^{-21} \mathrm{~m}
$$

Its rest charge radius should then be:

$$
r_{c_{e}}=\sqrt{2} r_{s_{e}}=2.970 * 10^{-21} \mathrm{~m}
$$

Obviously the charge of the electron has to be spinning faster than light:

$$
v=\frac{1}{2 m r_{c_{e}}} \hbar
$$

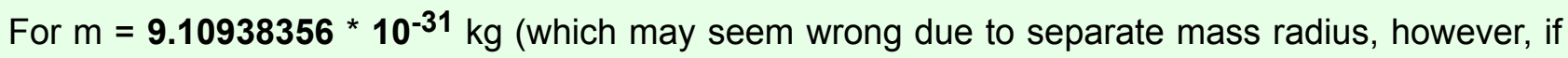
free electron is not naked, acquired real mass can be the charge mass shielding the mass of the maximum), this gives $v=9.745 * 10^{15} \mathrm{~m} / \mathrm{s}$.

This speed is the speed limit for particles in electron's space and it suggests that acquired real mass is of $U_{-1}$ scale or lower, making the spin momentum of the electron effectively the rotation of space, relative to standard scale.

The fact that imaginary mass is quantized by ${ }^{10} \mathrm{C}$ nucleus mass confirms the carbon-like nature of the Solar System equivalent on the standard scale, however, the magnitude of exchange of polarized (electromagnetic) potential for neutral gravitational potential suggests the Solar System is a scaled Bose-Einstein condensate of multiple atoms.

Note that the mass is equal to predicted $\mathbf{W}$ boson mass in some Electroweak models ${ }^{8}$.

From the calculated mass one can now obtain real part of Neptune's total mass: 


$$
\begin{gathered}
\frac{m_{r e}}{m_{i m g}}=\frac{m_{r e_{1}}}{m_{i m g_{1}}} \approx \frac{m_{r e_{1}}}{M_{U_{1}}} \\
m_{r e_{1}}=\frac{M_{e}}{m_{i m g}} M_{U_{1}}=5.60974244 * 10^{20} \mathrm{~kg}
\end{gathered}
$$

In the above, it was assumed that charge radius is equal to mass spin radius $\left(\mathbf{r}_{\mathbf{s}}\right)$ of the gravitational maximum. However, real charge radius is smaller.

If one assumes Earth's mass radius of the gravitational maximum is at the inner core boundary with gravity equal to Sun surface gravity $\left(274 \mathrm{~m} / \mathrm{s}^{2}\right)$, charge radius of Earth must be at a radius where gravity of the maximum is equal to half this value (this will be validated later):

$$
\begin{array}{r}
r_{c}=\sqrt{G M \frac{2}{274}}=\sqrt{\frac{G M}{137}}=1705704 m \\
M=\text { Earth's mass }=5.9723 * 10^{24} \mathrm{~kg} \\
\mathrm{G}=\mathbf{G}_{0}=\text { standard gravitational constant }=6.674 * 10^{-11} \mathrm{~m}^{3} / \mathrm{kgs}^{2}
\end{array}
$$

Using Q1.5, one can now calculate real mass component of the Earth:

$$
m_{r e}=\frac{r_{c}}{r_{a}} m_{i m g} \approx \frac{r_{c}}{r_{a}} M \approx 6.81 * 10^{19} \mathrm{~kg}
$$

\begin{tabular}{|c|c|c|}
\hline planet & equivalent standard $\mathbf{m}_{\text {img }}\left(\mathbf{G e V} / \mathbf{c}^{2}\right)$ & particle \\
\hline Saturn & 12.58 & 10 * charm quark, or 2 * charmed B meson \\
\hline Jupiter & 5.69 & ADM (asymmetric dark matter) particle ? \\
\hline Uranus & 57.87 & 10 * ADM ? \\
\hline Mars & 34.36 & $?$ \\
\hline Earth & 12 & $?$ \\
\hline Venus & 9.14 & $?$ \\
\hline Mercury & 12.13 & $?$ \\
\hline
\end{tabular}

$$
r_{\mathrm{a}}=\text { Earth's orbital radius }=149.6 * 10^{9} \mathrm{~m}
$$

This real mass will be further validated later. However, obtained charge radius is, as it will be shown later, the induced charge radius, rather than the primary or primordial charge radius.

Calculatimg $\mathbf{m}_{\mathbf{i m g}}$ for other planets hints that all may be consistent with condensates of standard particles:

There are no obvious candidates for terrestrial planets, and excluding Saturn and Neptune, even the candidates for outer planets may be questionable. 
However, there are various reasons for this - mass oscillation/excitation, unusual pairing (as in case of Neptune), unknown particle (having extremely short lifetimes on standard scale, unstable outside of atom), etc.

Multiples of atomic nuclei have also not been taken into account, which may be the most likely candidates, given the strong agreement of quantization of outermost particle mass with ${ }^{10} \mathrm{C}$ nuclei and the fact that, in a condensed state, an atomic nuclei is a viable equivalent of a particle (quantum of energy).

Two results for the velocity are in good agreement. Small difference can be attributed to uncertainty in vacuum energy density - a value of $9.79 * 10^{-27} \mathrm{~kg} / \mathrm{m}^{3}$ would yield the correct value.

Note that properties of space change inside the atom, this includes $\varepsilon_{0}$ and, consequently, $\hbar$ constant. Thus, velocities calculated using QM are generally not real (this velocity would produce a $1 / 3 \hbar$ orbital angular momentum in $\mathbf{Q M})$.

That properties of space change within the atom is obvious through the varying speed of light between different materials. Relative dielectric and magnetic constants should be interpreted as evidence for this photon can get absorbed and re-emitted in the atom, but what causes the delay?

If electrons have different energies on different orbits but have equal rest mass, energy must be stored in local space, varying with distance. If spin of the electron changes, energy is stored in its own space. All of these spaces are quantized, but using the same constants for all of them will generally not produce results matching reality. It may provide good and consistent results when measuring effects outside of the atom in consistent external space, but it gives a wrong image of its internal equilibrium structure.

From this one can also obtain the scaled speed of light:

$$
\begin{gathered}
\frac{v_{U_{0}}}{c_{0}}=\frac{v_{U_{1}}}{c_{1}} \\
c_{1}=\frac{v_{U_{1}}}{v_{U_{0}}} c_{0}=2.930445979 * 10^{6} \frac{\mathrm{m}}{\mathrm{s}}
\end{gathered}
$$

The result is in agreement with $\mathbf{c}_{\mathbf{1}}$ previously obtained from kinetic energy of the Solar System $(2.93$ * $\left.10^{6} \mathrm{~m} / \mathrm{s}\right)$. 


\section{Initial setup and regular disturbances}

Solar System is the product of inflation (likely through annihilation) of smaller scale particles or/and deflation [through annihilation] of larger scale particles.

Suppose that at the moment of annihilation the carbon atom was briefly ionized and its mass and charge were condensed into the core when it started inflating. With the electrons inflating along, eventually, the charge would separate from mass again.

The energy provided for transition between adjacent energy levels is generally higher than required, thus, the flattened carbon atom likely expanded to multiple times its current radii, then compressed to current size, trading charge area for neutral gravitational volume.

The atom nucleus in the process expanded up to the main asteroid belt, then compressed, leaving behind orbiting gravitons which collapsed to form terrestrial planets. The collapses were recorded in the Sun, forming discontinuities.

Note that the effect is the same even without initial ionization - in that case, discontinuities would be inflated along with the atom, rather than produced in the process.

In the transition from charged two-dimensional ring to three-dimensional sphere, equatorial spin momentum has been fragmenting and [due to spin decoupling] spreading to (forming) polar regions.

Latitude variable rotation may have been initially established as the product of conservation of momentum in such redistribution of mass, even if it now may be sustained differently.

Beside the long lived energy level changes, short lived (temporary) inflation/deflation of gravitational maximums will occur with the absorption/emission of [properly scaled] gravitational waves, which may be electrically polarized (electro-magnetic).

In case of dipole waves, absorption will induce separation of charges and collapse of a spherical form of the maximum into a two-dimensional ring form.

Such disturbances will generally occur at regular intervals, with periods generally increasing proportionally to the scale of the system and the scale of disturbance. On the scale of stellar systems, common minimum periods are on the order of millions of years. 
Changes in energy of the Solar System cannot be exempt from general oscillation and remain uniform over its lifetime.

For the Solar System, I hypothesize the existence of $\mathbf{3}$ periods (the arguments for which will be provided later, in this article, and in follow-up articles) for the first three orders of general oscillation:

1. $4.25 * 10^{9}$ years.

2. $25.7-25.92 * 10^{6}$ years,

3. $1.512 * 10^{6}$ years,

These are cycles of existence of the Solar System.

Only the 1st cycle may result in large scale horizontal energy level changes, but all these disturbances are sourced in gravitational stresses and have a strong effect on the evolution of the system (and all life within) - it is temporarily accelerated at the end of each cycle.

Large scale events are always preceded and superseded by smaller scale events so accelerated evolution may proceed for years on smaller scales before the actual disruption on larger scale occurs.

One may now attempt to calculate how much such disturbances last on the large (cataclysmic) scale.

With no change in energy level, orbital areal velocity of bodies, per Kepler's 2nd law, must remain constant and there should be no change in constitutional mass either.

With a temporary collapse of a gravitational maximum, escape velocity is extremely reduced and orbiting neutral real mass will be increasing orbital radii (although solid mass will generally preserve volume due to smaller scale electro-magnetic and neutral gravitational forces).

In order for this to be a temporary disturbance (no loss of entanglement), collapse must not exceed a specific time period - orbital period of the constituting mass.

Approximating gravitational maximum as a point maximum (linear ejection of mass from center) and assuming Sun's constitutional mass barycenter at the [inner] core radius at the time of collapse of the surface maximum, maximal allowed ejection distance $\mathbf{r}$ at the time the gravitational well is fully restored is:

$$
r=\frac{2 \pi r_{c}}{2}=\pi r_{c} \approx 0.63 R_{\odot}
$$

$$
\begin{array}{r}
R_{\odot}=\text { Sun radius }=695700 \mathrm{~km} \\
r_{\mathrm{C}}=\text { inner core radius }=1 / 5 R_{\odot}=139140 \mathrm{~km}
\end{array}
$$

Maximum time between the collapse and full restoration of the well is then: 


$$
t_{c}=\frac{2 \pi r_{c}}{v_{c}}=\frac{1}{f_{c}}=608272.5061 \mathrm{~s} \approx 7 \text { days }
$$

where $f_{c}\left(\mathbf{1 6 4 4} \mathrm{nHz}^{9}\right)$ is the rotation frequency of the Solar core.

Note the following:

$$
\frac{1}{v_{c}} * 10^{12}=\frac{1}{2 \pi r_{c} f_{c}} * 10^{12}=\frac{1}{2 \pi * 0.2 * 695700 * 1644 * 10^{-9}} * 10^{9}=695771 \mathrm{~km} \approx R_{\odot}
$$

suggesting that this should be satisifed:

$$
v_{c} * R_{\odot}=1 * 10^{12} \frac{m^{2}}{s}
$$

or, in terms of areal velocity of the core:

$$
v_{a}=\frac{1}{2} v_{c} r_{c}=\frac{R_{\odot}^{2} \pi}{5^{2} t_{c}}=1 * 10^{11} \frac{\mathrm{m}^{2}}{s}
$$

A hint of deeper entanglement between the Solar core and surface maximum (quantization).

In the context of $\mathbf{C R}$, evolution of systems is not a steady continuous process over all time, but a process with cyclic strong (cataclysmic) changes and a slow (weak) continuous evolution through the cycle.

In one of the follow-up articles, the collapse period of $\mathbf{7}$ days will be later correlated with Earth's evolution, showing that primordial theology [no matter how distorted and misinterpreted over the years] is based on true events and facts.

As I came to realize this I went outside, in despair still burdened by the thought. It was 2 past midnight, I lied on concrete, still entangled with a summer day.. Looking upon the heavens, once again for signs of confirmation I was not expecting to find - "a comet would suffice", I've told my self inside. Not a minute away, there it was, a comet passing right in that patch of the sky I've been absorbing with the eyes.

Enlightened by the dark, a thought emerged from my self.. Up until recent times my life was watched like a movie scripted by the dice thrown by chance, but now, now I did not believe in things anymore, I simply knew.. 
This life ain't a fairytale based on true events, but reality based on a fairytale... 


\section{Effects of mass and gravitational stresses on Keplerian motion}

Orbits of bodies in gravitationally bound systems should obey the following equation (orbital law):

$$
v^{2}=\frac{G M}{r}
$$

$$
\mathrm{G}=\text { gravitational constant }
$$

where $\mathbf{v}$ and $\mathbf{r}$ are orbital (Keplerian) velocity and radius, respectively, while $\mathbf{M}$ is the mass contained within the radius $\mathbf{r}$.

In planetary systems, most of the mass $\mathbf{M}$ is contained within the star, while in galaxies, it is mostly in central supermassive black holes.

However, in both systems, there are orbits at which the equation is apparently not satisfied - $\mathbf{v}$ is either higher or lower than expected for detected mass $\mathbf{M}$.

In galaxies, it is assumed that the discrepancy is caused by exotic gravitational mass - dark matter.

In planetary systems, spin of bodies does not obey the equation, but this is largely ignored (not considered as discrepancy), possibly due to current understanding of gravity and accepted theories on formation of planetary systems.

It is however, a legitimate question - why should a gravitationally bound mass in a galaxy obey the orbital law, while clouds of gas orbiting near the surface of a star should not (if most of $\mathbf{M}$ is below the surface)?

If the problem of gas is ignored because it is considered as constituent mass of the star, why are stars themselves not considered as constituent part of galaxies? And why would all constituent mass be exempt from the orbital law in the first place?

In CR, gravitational force of bodies with a distinct gravitational well may be largely provided by the gravitational maximum(s) so [ordinary] matter content (real mass) may be low.

Thus, a potential equivalent dark matter problem may exist in stars, planets, dwarf planets and larger moons (asteroids and comets are composites of smaller scale wells [held together in most part by electromagnetic force] so their spin momentum should not be Keplerian, even if their orbit around a body with a distinct maximum should). 
All bodies with a distinct gravitational well have a dark matter source (gravitational maximum), however, the addition (acquisition) of smaller scale matter (real mass) will shield the existence of the maximum, effectively decreasing imaginary mass content of the well.

Note that, in this exchange of dark gravitational potential for real gravitational potential, net gravitational force remains constant, but the capacity of the well (for real mass) is decreasing.

A body may also have multiple maximums, in which case, the outermost (surface) maximum will shield the existence of deeper maximums.

The shielding effect is not limited to the neutral gravitational component of general force, electromagnetic component may be shielded as well.

Thus, if there is no exchange of neutral gravitational potential for electro-magnetic potential, and if there are no changes in kinetic energy, despite the loss of matter, the gravity of a star should not change with age. The attraction remains, but its nature changes - from being mostly in its looks (real mass) to being mostly in its spirituality (dark matter), as in any living being.

Luminosity is thus, generally, a good measure of gravitational mass only if the well is at full capacity, otherwise it is only correlated with real mass and age.

The solution for terrestrial bodies lies in the loss of entanglement between space and matter orbitals due to interaction (collision) with other bodies, during formation of the body of matter.

Due to interaction of the atmosphere with a solid body beneath (or its origin), neither the gases of the atmosphere (or trapped particles from outer space interacting with the atmosphere) may obey the orbital law.

This suggests that even below a gas cloud rotating around a distinct maximum at non-Keplerian velocity there should be a solid core, at least in case of a neutral gas, however, angular component of velocity may be converted to radial and then to temperature.

Note that even if pressure from high temperature (kinetic energy) is balancing gravitational force, the thermodynamics (within the gas cloud) cannot break the orbital entanglement of the gas cloud as a whole.

If that gas is in the form of plasma (as in the case of Sun), it is more likely to be entangled with the charge component of a maximum (general force), which then should be the source of its non-Keplerian motion. 
The neutral gravitational equivalent of electro-magnetic influence on gas on the equator of the Sun can be calculated:

$$
\begin{array}{r}
v=v_{e}=\sqrt{\frac{G M_{2}}{r}}=1969.239615 \frac{\mathrm{m}}{\mathrm{s}} \\
\mathrm{v}_{\mathrm{e}}=\text { equatorial velocity of the Sun surface }
\end{array}
$$

which gives for the mass of the hypothetical neutral maximum:

$$
M_{2}=4.042341 * 10^{25} \mathrm{~kg}
$$

If the electro-magnetic component of the maximum would be exchanged for neutral gravitational component, the equatorial matter could remain entangled with such maximum.

The observed angular velocity could be interpreted as the evidence of spin change during the transition between vertical energy levels and transformation of electro-magnetic potential for neutral gravitational potential.

Suppose that entire potential was initially electro-magnetic but with an opposite spin. During transformation, Keplerian velocity component would be decreasing total angular velocity and, as the neutral component becomes larger than the electro-magnetic component, real mass would start spinning in another direction - aligned with Keplerian velocity. With complete transformation, real mass would have a Keplerian angular velocity.

However, with the exchange of potential and inflation of space, increasing gravity must be radially compressing orbitals, increasing density of real mass. If the compression is not isotropic and the mass is spiraling inwards (as expected for interaction of binaries at the event of annihilation), angular velocity (being exchanged for radial) will be decreasing from Keplerian with orbital radius.

This will be increasing pressure and temperature around the center which will balance the neutral gravitational force at equilibrium.

Angular velocity of matter around stars is thus generally proportional to a difference between neutral and electro-magnetic potential and, in magnitude, inversely proportional to temperature/density of real mass.

However, the stability of a gravitational maximum is proportional to its mass and inversely proportional to gravitational stress.

That gravitational stress affects the number of sunspots have already been shown ${ }^{10}$, and here I hypothesize that a sunspot pair is the result of a collapse of a quantum of a neutral gravitational surface 
maximum into a pair of [electrically] oppositely charged and relatively unstable spin maximums.

Note that the orbital radius of a sunspot pair is equal to the radius of the maximum before collapse.

Gravitational wells of planets, dwarf planets and major moons have been formed in the same way as sunspots.

Note also that the size of sunspots ranges from the size of a moon to the size of the biggest planet (Jupiter).

A neutral component of the naked gravitational maximum is dark matter, visible matter is real mass attracted to the gravitational well of such maximum. The velocity curves of the Sun and the Milky Way galaxy likely have the same solution - in the form of gravitational maximums and relativity of their nature due the exchange of polarized and non-polarized potential of general force.
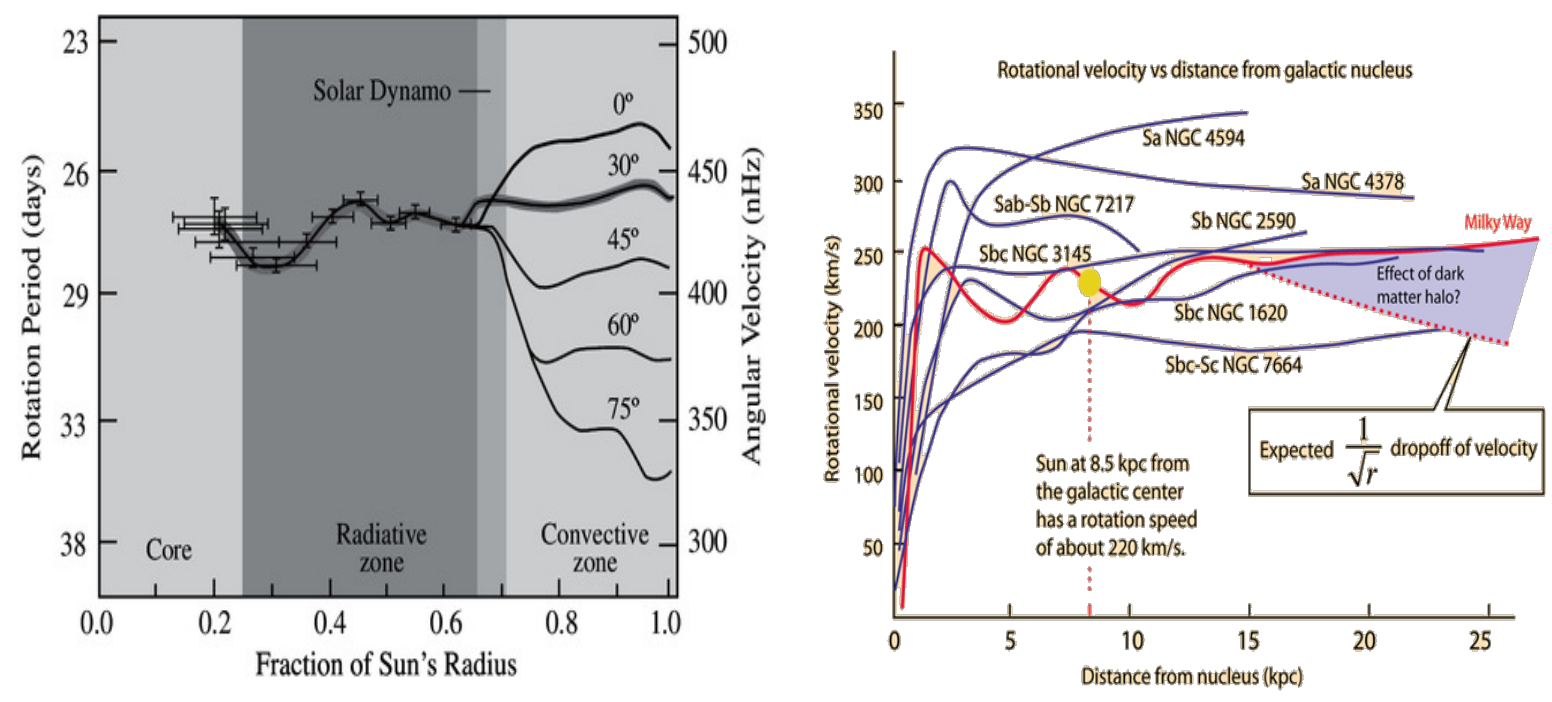

Fig. 1: left) internal rotation of the Sun ${ }^{11}$, right) rotation of spiral galaxies ${ }^{12}$

Rotation frequencies of the Sun (from the core up) and rotational velocities of several spiral galaxies are shown on Fig. 1.
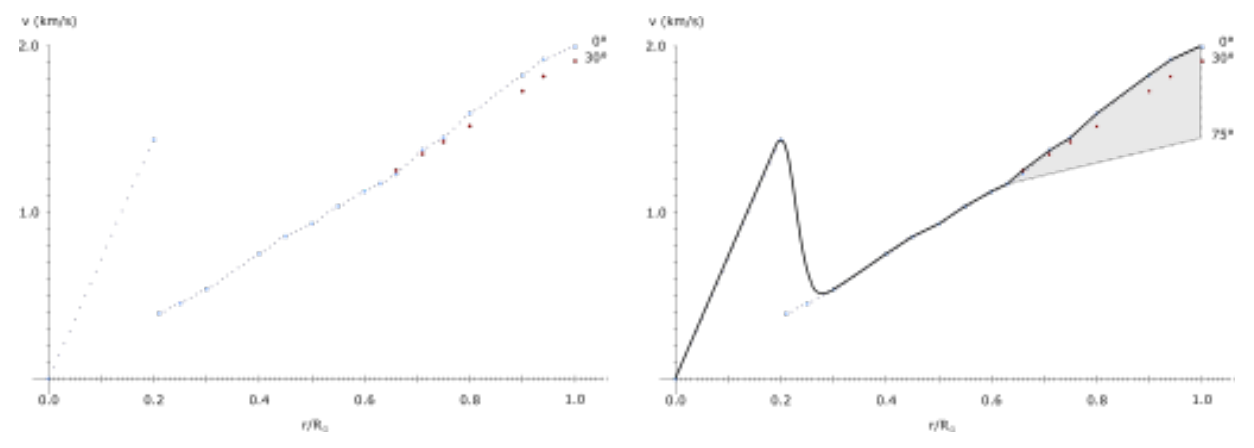
Fig. 2: Rotational velocities of the Sun

On the left, Fig. 2 shows the rotational velocities of the Sun based on rotation frequencies from two independent studies, one for the core $\left(r<\mathbf{0 . 2} \mathbf{R}_{\odot}\right)$ and other from the core up (black dots are interpolated values, red dots show velocities at $30^{\circ}$ latitude).

On the right, Fig. 2 shows the complete velocity curve (with interpolated connection between two curves) and dispersion of velocities (shaded area) due to differential rotation in the convective zone.

What is obvious from the figures is that Sun rotates like a composition of two solid bodies (diverging only in the polar regions of the convective zone), consistent with condensation of $\mathbf{U}_{\mathbf{1}}$ down and up quarks (energy levels) into two ground states (+1s/-1s).

Assuming the Sun is not solid anywhere (as expected in conventional theories), it should be mainly composed of plasma.

However, there is a possibility that fusion in stars operates differently (or at least has a secondary component) - through the bombardment of solid (or solid-like) material with particles produced in the radiative zone. These may be high energy photons produced through matter/anti-matter annihilation and/or high temperature of plasma.

Evidently, velocity curve of the Sun is similar to a typical velocity curve of a spiral galaxy - in both cases there is an initial sharp increase in velocity in the core, followed by a decline, with each next increase in velocity being less steep than the previous one. Note that latitude dependent differential rotation may also be common at specific places in galaxies too.

If the spin momentum of the Sun is effectively immune to [large scale] collisions (even if the core would be solid, everything approaching the Sun is vaporized before reaching the surface), the only disturbance of Keplerian orbits must come from incomplete conversion of electro-magnetic potential and increase of temperature.

Assuming that orbital velocity is decreasing (from Keplerian velocity) proportionally to electro-magnetic potential, as hypothesized, orbital velocity of plasma should keep increasing with radius until it becomes equal to Keplerian velocity, beyond which point there should be no accumulation of charge and the radial component of the solar wind should dominate.

Using approximation of the velocity/radius dependence based on the velocity curve of the Sun (up to $130000 \mathrm{~km}$ from surface ${ }^{13}$ ), and equalizing with orbital law: 


$$
v=\frac{2533.61175}{1.18686-0.1}\left(\frac{r}{R_{\odot}}-0.1\right)=\sqrt{\frac{G M_{\odot}}{r}}
$$

one obtains the orbit of such discontinuity:

$$
r=32.8 R_{\odot}=22.826 * 10^{6} \mathrm{~km} \approx 33 R_{\odot}
$$

First results from the Parker solar probe indicate a significant rotational velocity of the solar wind around $40 \mathbf{R}_{\odot}$, peaking at the closest approach. The results indeed indicate a high probability of a maximum velocity around $\mathbf{3 3} \mathbf{R}_{\odot}$ in case a rigid rotation of the solar wind is maintained up to that point.

Rigid rotation is a consequence of relative cancellation of neutral and electro-magnetic influence on angular velocity, making it dependent on real mass (solar wind) density (pressure) which for particle orbitals falls of proportionally to distance $r$ (number of particles per $2 \pi r$ is constant).

Note that, even without rigid rotation, the discontinuity should occur at the point where velocity becomes equal to Keplerian velocity, otherwise, higher velocity would indicate dark matter presence - another maximum.

Note that $33 \mathbf{R}_{\odot}$ is equal to $0.1 \mathrm{MAU}$ (Sun-Mars distance), while the above equation gives $0.1 \mathbf{R}_{\odot}$ for $v=\mathbf{0}$. This correlation of the radius of the Sun with the orbit of Mars is not a coincidence - Mars is the outermost positive charge of the $\mathbf{U}_{1} \cdot{ }^{10} \mathrm{C}$ atom (Solar System).

If the same is applied to the core of the Sun, the velocity at $\mathbf{0 . 2} \mathbf{R}_{\odot}$ should be equal to Keplerian velocity. Here, however, this velocity is the sum of Keplerian velocity of the surface maximum and a core maximum. For a surface maximum at $\mathbf{R}_{\odot}$ :

$$
v=s \sqrt{\frac{G M}{0.2 R_{\odot}}}+s s_{\frac{G M_{\odot}}{R_{\odot}{ }^{2}} \frac{\left(0.2 R_{\odot}\right)^{2}}{R_{\odot}{ }^{2}} 0.2 R_{\odot}}=s \sqrt{\frac{G M}{0.2 R_{\odot}}}+s \sqrt{G M_{\odot} \frac{\left(0.2 R_{\odot}\right)^{3}}{R_{\odot}{ }^{4}}}
$$

$\mathrm{s}, \mathrm{s}_{\odot} \in\{-1,1\}$

where $\mathbf{M}$ is the mass of the core maximum, $\mathbf{s}$ is the spin polarization of gravity of the core maximum and $\mathbf{s}_{\odot}$ is the spin polarization of gravity of the surface maximum.

Equalizing this velocity with measured velocity at the core discontinuity:

$$
v=2 \pi * 0.2 R_{\odot} * f=2 \pi * 0.2 R_{\odot} * 1644 * 10^{-9}=1437.2545 \frac{\mathrm{m}}{\mathrm{s}}
$$


and setting spin polarization positive for counter-clockwise rotation [of the surface maximum], gives $\mathbf{s}=\mathbf{- 1}$ and gravitational mass of the core roughly $3 / 2$ the Jupiter mass:

$$
M=2.951797 * 10^{27} \mathrm{~kg}
$$

which gives mean core density of:

$$
\rho=261.602486 \frac{k g}{m^{3}}
$$

implying the primary gravitational mass of the Sun is above the core. Difference in mass between the core and outer layers is roughly equal to the mass difference between inner and outer planets.

For the ratios to be equal, core mass must be 3 times higher, which indicates that space has been stretched (compressed, relative to core) from $\mathbf{0 . 2 8 6} \mathbf{R}$ to $\mathbf{0 . 2} \mathbf{R}$. Modifying the equation for Keplerian velocity accordingly would give the initial mass $\left(8.90211033\right.$ * $\left.10^{27} \mathrm{~kg}\right)$ :

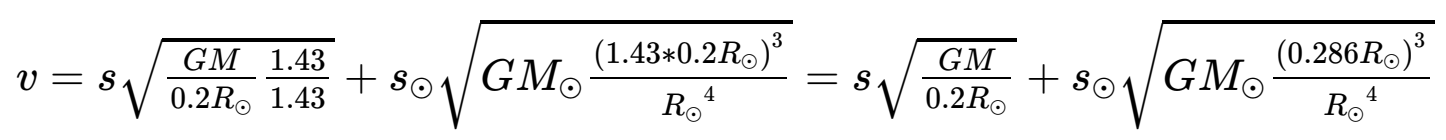

Radius independent Keplerian velocities, like those at the outskirts of galaxies, are the effect of stretched space between maximums.

With shorter distance between maximums, minimum is more localized and changes in velocity are sharper.

Apparently, such stretching occurs in the Sun too.

Note that the equation $\mathbf{S 1 . 1}$ is defined by the straight line passing through $\mathbf{0 . 1} \mathbf{R}_{\odot}$ and $\mathbf{1 . 1 8 6 8 6} \mathbf{R}_{\odot}$, so if one assumes that, without space stretching, the defining points would be $\mathbf{0 . 0} \mathbf{R}_{\odot}$ and $1.0 \mathbf{R}_{\odot}, \mathbf{0 . 2 8 6} \mathbf{R}_{\odot}$ is the sum of translation of both points in radial direction due to stretching.

Note also that, if Sun looses all outer mass with the collapse of the outer maximum, with leftover mass roughly equal to initial core mass the Solar System becomes geocentric.

This stretching of space is evident on Fig. 3 in the sharp increase of velocity from $0.286 \mathbf{R}$ to $0.2 \mathrm{r}$. To conserve momentum, this increase in velocities in the inner half had to decrease velocities in the outer half of the Sun, up to $1.18686 \mathbf{R}_{\odot}$. 


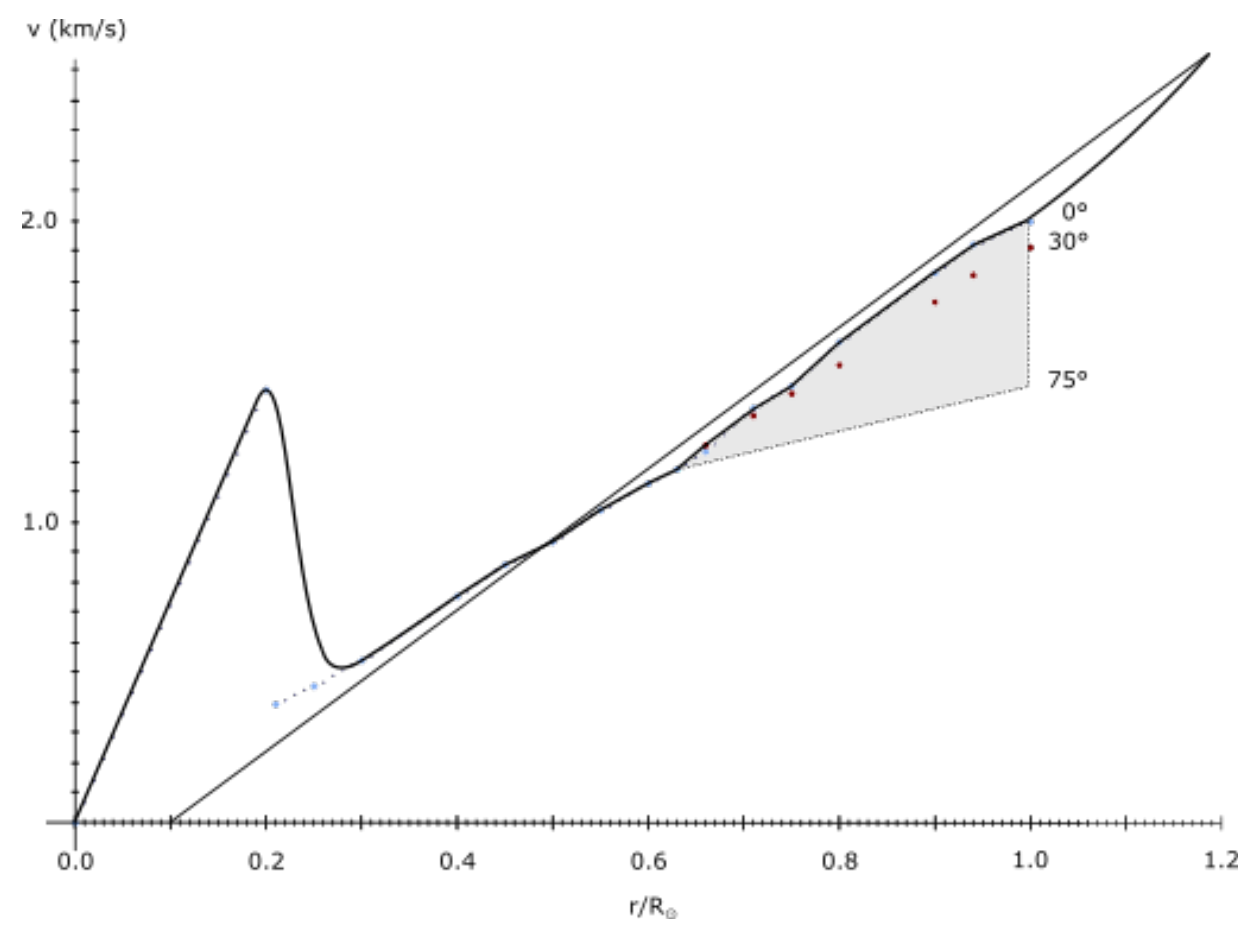

Fig. 3: Rotational velocities of the Sun and near corona

Note that slower polar convective rotation could be the result of loss of shielding of the core maximum [charge] due to conversion of potential of the surface maximum (convergence from spherical to ring form).

Due to zero gravity at $\approx 0.2 \mathrm{R}$ and gravitational attraction of the surface maximum, any particle escaping the core will overcome escape velocity at the surface of the Sun (if not slowed down by other particles). The same is true for the other direction. Thus, the orbitals of particles at the discontinuity must be highly unstable and it should be the area of lowest [real mass] density.

However, gravitational stress can cause the collapse of the surface maximum. If that stress is low (insufficient for full collapse), the maximum will be fragmenting and collapsing into quanta of smaller charged maximum pairs, starting in polar regions (and, without further increase of stress, limited to polar regions).

At these places (sunspots), gravitational escape velocity is decreased allowing higher bandwidth of escaping mass, although significant transverse velocity component will exist, especially for charged particles.

Note that orbitals at polar regions seem to be entangled with the core.

Strong entanglement between [quark] pairs may exist between the core and surface, it is also possible that gravitational stress is adding energy to such entanglement and inflating maximums of such pairs (creating wormholes). 
In that case space is stretched from the cure to the surface (at sunspots) entangling orbital velocities but also being fixed to specific latitude by magnetic field lines (shielding inclined velocity component), the period of rotation of such plasma on the surface would be equal to:

$$
T=\frac{2 \pi R_{\odot}}{v}=3041363 s=35.2 \text { days }
$$

which is the rotation above $75^{\circ}$ latitude and should be the location of sunspot creation (inflation) at surface. Note that, once the orbital entanglement is lost, being charged, the sunspots will drift along the magnetic field lines.

The specific core discontinuity radius is the result of equilibrium between the outer gravitational force and induced vacuum force (forces cancel near the discontinuity).

The core gravitational maximum of the Sun might be the effect of vacuum, but, likely, all gravitational maximums are the result of vacuum induction and quantization.

In example, similar to inner Solar planets, the stars of a particular arm of a spiral galaxy could be the result of vacuum collapse into smaller quanta (maximums).

One might understand the creation of vacuum as stretching of space and decrease of density, but no space can be absolutely empty. Thus, if one is stretching space, one is also inflating smaller scale maximums.

The stretching of space between galaxies must result in creation of dark matter filaments between them. Intergalactic and galactic dark matter is thus likely created by dark energy, which is nothing else but a gravitational force of a large scale maximum well [of dark matter], expanding vacuum, just like it happens in the Sun.

Due to discretization of stable energy [levels], with enough energy applied into vacuum creation, the inflation will result in [relatively] permanent maximums of larger scale.

Vacuum inflation may be most likely in annihilation events, due to high symmetry and energy localization. However, stretching of space between strongly entangled particles can also result in permanently inflated particles (as in quark/anti-quark pairs).

If the inflated particles are always of equal species to the original particles, evidently the [private] space of such particles is composed of the same particles but of lower scale. 
In case of annihilation, the stretched (inflated) space might not be the space of annihilating pair, rather the underlying space, making the product of inflation highly dependent on the point of interaction.

It may be more appropriate to state that, rather than being stretched in between, space is compressed at maximums. Similar to the 1st law, one could then construct another law:

\section{Space remains at constant density unless acted upon by gravitational force.}

Thus, even if it may appear that, once deformed, no force is necessary to act on bodies in space to accelerate their radial motion relative to the sources of gravity, force (energy) is necessary to maintain such state of space.

As everything must conform to general oscillation, some force is always present, with relative magnitude and distance it is acting upon.

The speed of motion (radiation) will depend on density of space and, if gravitational force is limited, there will be a speed limit on motion. However, constancy of density is relative and even density is relative to the scale of the 1 st order observer, or, more precisely, the strength of its entanglement with such space.

Absolute, and absolutely invariant limits are impossible.

In any case, it seems that everything must be mirrored, and when it appears that is not the case, the cause is simply a large distance - in scale of space/time.

If the point of interaction of an annihilating pair imparts energy to the pair in highly asymmetric manner, the inflation would result in a pair of maximums of different scale (in fact, one of the particles could even be deflating).

Thus, a possibility exists that even a proton and electron particles are the result of an anomaly in annihilation of particle/anti-particle pairs of equal scale.

Note that a gravitational maximum must have a radius - a point maximum would imply infinite gravity and no possibility for containment of smaller maximums.

Somewhere around the discontinuity, conditions may even be suitable for standard life. Note that the radius of the core is almost 22 times Earth radius, if density is not isotropic, smaller bodies might be orbiting inside. 
Considering the momentum of the Solar System barycenter, density should not be isotropic. 


\section{Quantization of momentum}

Previous works based on Titius-Bode law have shown that planetary orbits are quantized ${ }^{14}$ :

$$
r=a e^{2 \lambda n}
$$

More recently it has been shown that distances and orbital periods are consistent with quantized scaling ${ }^{15}$, rather than logarithmic.

Here, it will be shown that, taking into account the splitting of momentum inside the atom, angular momentum is quantized too, as well as surface gravity.

If QM cannot describe the Solar System as an atom, it is QM that should be revised, not reality.

Gravitational maximums (event horizons) are sphere surfaces with a specific radius. If one expects real mass to be similar between the planets, difference between the planets must be in radii of these maximums.

Thus, surface gravity should be a quantized parameter, inversely proportional to real mass capacity of the gravitational well. For outer planets, radius of the maximum is hypothesized to be equal to what is currently defined as the surface radius ( 1 bar pressure level).

When quantized, orbital angular momentum satisfies the following equation:

$$
m v r=n \hbar
$$

where $\hbar$ is a constant, $\mathbf{n}$ is a positive integer number and $\mathbf{m}, \mathbf{v}, \mathbf{r}$ are components of orbital angular momentum - mass, velocity and radius, respectively.

Using total mass of the planet for $\mathbf{m}$ will not reveal quantization. In example, using Neptune's mass of $1.02413 * 10^{26} \mathrm{~kg}$ and setting $\mathrm{n}$ to 5 :

$$
m v r=5 \hbar=2.499714508 * 10^{42} \mathrm{Js}
$$

one obtains the scaled $\hbar$ (Planck's) constant for outer planets:

$$
\hbar=\hbar_{m_{2}}=4.999429016 * 10^{41} \approx 5 * 10^{41} \mathrm{Js}
$$

While the result is certainly interesting, the same $\hbar$ will not produce quantized momentums for other planets (it needs to be scaled). 
The mass which does produce quantized angular momenta is, as previously established (equation Q1.4), the real part of total mass.

However, surface gravity for outer planets can be calculated using the obtained $\hbar$, Neptune's total mass $\mathbf{M}_{\mathbf{N}}$ and surface gravity $\mathbf{g} \mathbf{N}$ :

$$
g=\frac{v r}{n \hbar} M_{N} g_{N}
$$

\begin{tabular}{|l|l|l|l|l|l|l|l|l|}
\hline $\mathbf{n}$ & planet & $\begin{array}{l}\text { orbital } \\
\text { velocity v } \\
(\mathrm{m} / \mathrm{s})\end{array}$ & $\begin{array}{l}\text { orbital } \\
\text { radius } \mathrm{r} \\
\left(10^{6} \mathrm{~km}\right)\end{array}$ & $\begin{array}{l}\text { total mass } \\
\mathrm{M}\left(10^{24}\right. \\
\mathrm{kg})\end{array}$ & $\begin{array}{l}\text { required total } \\
\text { mass }\left(\mathbf{1 0}^{24} \mathrm{~kg},\right. \\
\text { based on } \\
\text { Neptune })\end{array}$ & $\begin{array}{l}\text { calculated } \\
\text { gravity } \mathrm{g} \\
\left(\mathrm{m} / \mathrm{s}^{2}\right)\end{array}$ & $\begin{array}{l}\text { gravity } \\
\left(\mathrm{m} / \mathrm{s}^{2}\right)\end{array}$ & $\begin{array}{l}\text { acceleration } \\
\left(\mathrm{m} / \mathrm{s}^{2}\right)\end{array}$ \\
\hline 5 & Neptune & 5430 & 4495.06 & 102.413 & 102.413 & 11.15 & 11.15 & 11.00 \\
\hline 5 & Uranus & 6800 & 2872.46 & 86.813 & 127.975698 & 8.92 & 8.87 & 8.69 \\
\hline 3 & Saturn & 9680 & 1433.53 & 568.340 & 108.0835294 & 10.565 & 10.44 & 8.96 \\
\hline 1 & Jupiter & 13060 & 778.57 & 1898.190 & 49.16766277 & 23.225 & 24.79 & 23.12 \\
\hline
\end{tabular}

In the table above, required total mass is the total mass (gravitational energy) required to satisfy the quantization by standard QM (showing how far it is from reality).

The protons and electrons are parts of two different universes (as difference in scale suggests), so one should use a different $\hbar$ constant for terrestrial planets (proton partons).

The angular momentum of Mercury $\left(m=M=3.3011 * 10^{23} \mathrm{~kg}\right)$ :

$$
m v r=5 \hbar=9.053654959 * 10^{38} \mathrm{Js}
$$

gives the scaled $\hbar$ constant for inner planets:

$$
\hbar=\hbar_{m_{1}}=1.810730992 * 10^{38} \mathrm{Js}
$$

\begin{tabular}{|c|c|c|c|c|c|c|c|}
\hline$n$ & $\begin{array}{l}\text { planet } \\
\text { (entanglement) }\end{array}$ & $\begin{array}{l}\text { orbital } \\
\text { velocity v } \\
(\mathrm{m} / \mathrm{s})\end{array}$ & $\begin{array}{l}\text { orbital } \\
\text { radius r } \\
\left(10^{6} \mathrm{~km}\right)\end{array}$ & $\begin{array}{l}\text { total } \\
\text { mass } \\
\left(10^{24} \mathrm{~kg}\right)\end{array}$ & $\begin{array}{l}\text { required total mass } \\
\left(10^{24} \mathrm{~kg} \text {, based on }\right. \\
\text { Mercury) }\end{array}$ & $\begin{array}{l}\text { calculated } \\
\text { gravity g } \\
\left(\mathrm{m} / \mathrm{s}^{2}\right)\end{array}$ & $\begin{array}{l}\text { gravity } \\
\left(\mathrm{m} / \mathrm{s}^{2}\right)\end{array}$ \\
\hline
\end{tabular}

Surface gravity for inner planets, using obtained $\hbar$, Mercury mass $\mathbf{M}_{\mathbf{M}}$ and gravity $\mathbf{g}_{\mathbf{M}}$ :

$$
g=\frac{v r}{n \hbar} M_{M} g_{M}
$$




\begin{tabular}{|c|c|c|c|c|c|c|c|}
\hline 5 & $\begin{array}{l}\text { Mercury } \\
\text { (Neptune) }\end{array}$ & 47360 & 57.91 & 0.330 & 0.33011 & 3.70 & 3.70 \\
\hline 3 & Venus (Uranus) & 35020 & 108.21 & 4.868 & 0.1433480042 & 8.52 & 8.87 \\
\hline 3 & Earth (Saturn) & 29780 & 149.6 & 5.972 & 0.1219323384 & 10.02 & 9.798 \\
\hline 10 & Mars (Jupiter) & 24070 & 227.92 & 0.642 & 0.3300619099 & 3.70 & 3.71 \\
\hline
\end{tabular}

Quantization can be shown without using mass (directly), through the volumetric space-time momentum (gravitational momentum):

$$
g v r=n h\left[\frac{m^{3}}{s^{3}}\right]
$$

With $\mathbf{h}$ obtained from above, substituting mass with gravity, the equation for gravity becomes:

$$
g=\frac{v r}{n h} g_{0}^{2}
$$

where $g_{0}$ is the gravity of Neptune, or, in case of terrestrial planets, the gravity of Mercury, and it yields the same results.

While the second equation will yield the correct results for gravity, the equation gvr = nh will not, showing the inverse coupling of gravity to momentum:

$$
\frac{1}{g} v r=n h[m s]
$$

This gives, for outer planets:

$$
h=h_{g_{2}}=4.378148126 * 10^{14} \mathrm{~ms}
$$

for inner planets:

$$
h=h_{g_{1}}=1.482496 * 10^{14} \mathrm{~ms}
$$

Now, one can couple mass with gravity:

$$
\begin{gathered}
m v r=n \hbar_{m}, \frac{1}{g} v r=n h_{g}, \hbar_{m g}=\frac{\hbar_{m}}{h_{g}} \\
g=\frac{v r}{n h_{g}}=\frac{n \hbar_{m}}{m} \frac{1}{n h_{g}}=\frac{1}{m} \frac{\hbar_{m}}{h_{g}} \\
g=\frac{\hbar_{m g}}{m},
\end{gathered}
$$


and obtain relation to Sun's gravity:

$$
\begin{gathered}
r=\frac{n \hbar_{m}}{m v}=\frac{g n h_{g}}{v} \\
r^{2}=n^{2} \hbar_{m} h_{g} \frac{g}{m v^{2}} \\
\frac{m r}{g} \frac{4 \pi^{2} r^{3}}{T^{2}}=n^{2} \hbar_{m} h_{g}\left[k g m^{3}\right] \\
m r^{3} \frac{g_{S}}{g}=n^{2} \hbar_{m} h_{g}\left[k g m^{3}\right] \rightarrow v^{2}=r g_{S} \\
m^{2} r^{3} g_{S}=n^{2} \hbar_{m}^{2}\left[\frac{k g^{2} m^{4}}{s^{2}}\right]
\end{gathered}
$$

where $\mathbf{g s}$ is the gravity of Sun at orbital radius $\mathbf{r}$.

For outer planets:

$$
\hbar_{m g}=\hbar_{m g_{2}}=1.14190495 * 10^{27} \frac{\mathrm{J}}{\mathrm{m}}=1.14190495 * 10^{27} \mathrm{~N}
$$

For inner planets:

$$
\hbar_{m g}=\hbar_{m g_{1}}=1.221407 * 10^{24} \mathrm{~N}
$$

The above $\hbar_{\mathrm{mg}}$ constants are based on total mass, for real mass, the quantum of gravitational force $\left(\hbar_{\mathbf{m g}}\right)$ may be treated as invariant between inner and outer planets (with properly defined surface gravity $\mathbf{g}$ ):

$$
\hbar_{m g}=6.968267285 * 10^{20} \mathrm{~N}
$$

Small discrepancies in gravity stem mainly from the oscillation of surface gravity. Note, for example, with rotation taken into account $\left(g_{N}=11.0 \mathrm{~m} / \mathrm{s}^{2}\right)$ the calculated gravity for Saturn would match exactly the measured value of $\mathbf{1 0 . 4 4}$ (which is the value without rotation!). On the other hand, the gravity of Jupiter with rotation closely matches the calculated value (without rotation). This confirms that the definition of surface relative to fixed pressure ( 1 bar in this case) is appropriate for outer planets but should oscillate (cycle) between planets to take into account fossilization of a previous maximum in rotation period.

For terrestrial planets surface gravity is defined unrelated to pressure, as gravity at ground (sea) level. The calculated value matches Venus gravity at the transition zone between mesosphere and thermosphere.

For Earth, the value matches the transition zone between upper and lower mantle, or, if one 
calculates with constant mass, it is, just like in the case of Venus, the value of height of the mesosphere/thermosphere transition zone, but negative (below surface). So, here too, the cyclic nature of surface gravity is evident.

The constants $\mathbf{h}(\hbar)$ and $\mathbf{G}$ (gravitational constant) are scale dependent, but they also must oscillate. The above results could thus be interpreted as due to oscillation of energy of space (as $\mathbf{h} / \mathbf{G}$ directly depend on it).

This oscillation may be, for the electron, confined to the atom, at least at non-condensing temperatures.

Another interpretation for the excitations of $\mathbf{G}$ is the absorption of large scale external gravitational waves, however, these cannot explain the confinement of the oscillation to atoms.

In any case, when comparing small scale atoms with large scale atoms (ie. planetary systems), one must not only choose a proper reference frame and take into account the possible effects of measurement, but resolve the issues of QM - make constants (properties of space) relative, with proper attribution of relativistic effects.

The fact that similar planets (Venus/Earth, Uranus/Neptune) share the energy level (n) fits well with the quantum hypothesis.

The relative high excitation of Mars $(\mathbf{n}=10)$ and no excitation of Jupiter $(\mathbf{n}=1)$ indicates the system is in 6p4n state.

Note that the following should be satisfied (with oscillations in superposition):

$$
\frac{N}{P} \frac{\hbar_{m_{2}}}{\hbar_{m_{1}}}=\left(1-\frac{h_{g_{1}}}{h_{g_{2}}}\right) \frac{\hbar_{m_{2}}}{\hbar_{m_{1}}}=\frac{m_{p}}{m_{e}}
$$

where $\mathbf{m}_{\mathbf{p}}, \mathbf{m}_{\mathbf{e}}$ are masses of standard proton and electron, respectively. The factor N/P is the ratio of neutrons to protons in the Solar System:

\begin{tabular}{|c|c|c|c|}
\hline base & state & N/P & notes \\
\hline Carbon & $6 p 4 n$ & $\begin{array}{l}4 / 6= \\
2 / 3\end{array}$ & $\begin{array}{l}\text { Mercury } 3.7(0.25 \mathrm{MAU}, \mathrm{n}=5) \text {, Venus } 8.87(0.5 \mathrm{MAU}, \mathrm{n}=3) \text {, Earth } 9.798(0.66 \mathrm{MAU}, \mathrm{n}=3) \text {, } \\
\text { Mars } 3.71(1 \mathrm{MAU}, \mathrm{n}=10)\end{array}$ \\
\hline Boron & $5 p 5 n$ & $5 / 5=1$ & $\begin{array}{l}\text { Mercury B } 3.32(0.2 \mathrm{MAU}, \mathrm{n}=5) \text {, Venus/Earth A } 5.25(0.5 \mathrm{MAU}, \mathrm{n}=5) \text {, Earth B/Mars } 6.43(0.75 \\
\text { MAU, n=5) }\end{array}$ \\
\hline Beryllium & $4 p 6 n$ & $\begin{array}{l}6 / 4= \\
3 / 2\end{array}$ & Venus/Earth A 1.85 (0.25 MAU orbit, n=10), Earth B/Mars 37.1 (1 MAU, n=1) \\
\hline
\end{tabular}


This shows direct entanglement of standard proton and electron mass with planetary mass and configuration.

As all constants, constant masses of standard protons and electrons are a superposition of oscillation. As with the $\hbar$ constant, the oscillation can be detected on large scale.

On standard $\left(\mathbf{U}_{\mathbf{0}}\right)$ scale, proton to electron mass ratio is:

$$
\frac{m_{p}}{m_{e}}=1836.15267343(11)
$$

On $U_{1}$ scale:

$$
\begin{gathered}
\frac{N}{P} \frac{\hbar_{m_{2}}}{\hbar_{m_{1}}}=1840.66694172611441 \\
\left(1-\frac{h_{g_{1}}}{h_{g_{2}}}\right) \frac{\hbar_{m_{2}}}{\hbar_{m_{1}}}=1826.09096003909666
\end{gathered}
$$

From these, the value of superposition might be obtainable using the EH operator, ie. using 12/4 for the 1st order approximation:

$$
\begin{aligned}
& E H_{12 / 4}(, \lambda)+\left(1-\frac{h_{g_{1}}}{h_{g_{2}}}\right) \frac{\hbar_{m_{2}}}{\hbar_{m_{1}}}=\frac{m_{p}}{m_{e}}=1836.182024284 \\
& \lambda=\frac{N}{P} \frac{\hbar_{m_{2}}}{\hbar_{m_{1}}}-\left(1-\frac{h_{g_{1}}}{h_{g_{2}}}\right) \frac{\hbar_{m_{2}}}{\hbar_{m_{1}}}=\left(\frac{h_{g_{1}}}{h_{g_{2}}}+\frac{N}{P}-1\right) \frac{\hbar_{m_{2}}}{\hbar_{m_{1}}}
\end{aligned}
$$

The transition from $6 p 4 n$ to $5 p 5 n$ state includes:

- collapsing (vertical) scale,

- loss of one outer gravitational maximum (death of Neptune electron), dead matter remains,

- Mars' gravitational maximum fusing with one of Earth's gravitational maximums,

- fusion of Venus' gravitational maximum with remaining Earth's gravitational maximum,

- Mercury loosing one gravitational maximum,

- life possibly changing base to boron,

- formation of a new dwarf planet in the main asteroid belt, 
- space between planets expanding (Solar System expanding),

- Solar System increasing orbital momentum (velocity), decreasing spin momentum,

- spin momentum of planets increasing.

The transition from $5 p 5 n$ to $4 p 6 n$ state includes:

- scale collapse stop,

- loss of one outer gravitational maximum (Uranus e), dead matter remains,

- significant increase of Mars' gravity,

- death of Mercury, dead matter remains,

- significant increase of Venus' real mass, decreasing surface gravity,

- no complex surface life,

- formation of a new dwarf planet in the main asteroid belt,

- further expansion of space between planetary orbits,

- further increase of orbital momentum (velocity), decreasing spin momentum,

- further increase of planetary spins.

\subsection{Proper quantization in QM}

If one wants to compare the Solar System with a room temperature equivalent of a carbon atom in the context of QM, one must reduce the effects of exchange of em potential with neutral gravitational potential due to condensation and lepton oscillation.

In that case, real mass component of the total initial momentum (Q1.3, Q1.4), which is equal (relatively, but difference is negligible) between bound electrons, is the correct mass to be used in comparison.

Total initial momentum is the angular momentum, it is quantized and for all electrons in ground state should be equal to:

$$
m_{r e} v_{t o t} r_{a}=\frac{1}{2} \hbar
$$

However, generally, total momentum is the sum of orbital and spin components.

Each quantum sub-shell may contain up to 2 electrons. If these are in condensed (bosonic) form, their momentums are strongly coupled, they will behave as a single body, and the proper equation for the magnitude 
of total angular momentum per sub-shell is:

$$
\begin{gathered}
m_{r e} v_{t o t} r_{a}=\sqrt{l(l+1)} \hbar+s \hbar \\
v_{t o t}=v_{a}+v_{s}=v_{a}+\frac{2 \pi R_{s}}{T_{s}}
\end{gathered}
$$

$\mathbf{R}_{\mathrm{S}}=$ spin radius

$\mathbf{T}_{\mathrm{S}}=$ spin rotation period

where $\mathbf{s}$ is the total [magnetic] spin of electrons in a sub-shell.

Note that spin momentum components [of electrons] non-parallel to the axis of quantization are annihilated. Spin momentum magnitude $s$ can thus have the following values: $0, \pm 1, \pm 1 / 2$.

Since the value of $\mathbf{m}_{\mathbf{r e}}$ here is constant, its value is irrelevant to prove $\mathbf{Q M}$ equivalent quantization. For the sake of argument, let it be equal to $7 * 10^{19} \mathrm{~kg}$.

Since Jupiter has to be in $2 \mathrm{e}$ configuration (even if Solar System would not be the carbon [equivalent] atom), it is appropriate to derive $\hbar$ from its momentum.

Assuming $\mathbf{n}=\mathbf{1}$ (as expected) for Jupiter, I must be equal to $\mathbf{0}$, with s equal to $\mathbf{1}$, the $\hbar$ is:

$$
\hbar=m_{r e} v_{t o t} r_{a}=1.382 * 10^{36} \mathrm{Js}
$$

\begin{tabular}{|c|c|c|c|c|c|c|c|c|c|c|}
\hline $\mathbf{n}$ & configuration & I & $\mathbf{s}$ & planet & $\begin{array}{l}\text { orbital } \\
\text { velocity } v_{a} \\
(\mathrm{~m} / \mathrm{s})\end{array}$ & $\begin{array}{l}\text { orbital } \\
\text { radius ra } \\
\left(10^{6} \mathrm{~km}\right)\end{array}$ & $\begin{array}{l}\text { spin } \\
\text { velocity } \\
\mathbf{v}_{\mathbf{s}}(\mathrm{m} / \mathrm{s})\end{array}$ & $\begin{array}{l}\text { spin } \\
\text { radius } \\
R_{s}(\mathbf{k m})\end{array}$ & $\begin{array}{l}\text { spin } \\
\text { rotation } \\
\text { period Ts } \\
\text { (h) }\end{array}$ & $\begin{array}{l}\text { calculated } \\
\hbar \text { (Js) }\end{array}$ \\
\hline 5 & $1 e$ & 1 & $1 / 2$ & Neptune & 5430 & 4495.06 & 2668 & 24622 & 16.11 & $\begin{array}{l}1.3310 \text { * } \\
10^{36}\end{array}$ \\
\hline 5 & $1 e$ & 1 & 0 & Uranus & 6800 & 2872.46 & 2568 & 25362 & 17.24 & $\begin{array}{l}1.3319 \text { * } \\
10^{36}\end{array}$ \\
\hline 3 & $2 e$ & 1 & 0 & Saturn & 9680 & 1433.53 & 9538 & 58232 & 10.656 & $\begin{array}{l}1.3636 \text { * } \\
10^{36}\end{array}$ \\
\hline 1 & $2 e$ & 0 & 1 & Jupiter & 13060 & 778.57 & 12293 & 69911 & 9.9250 & $\begin{array}{l}1.3817 \text { * } \\
10^{36}\end{array}$ \\
\hline
\end{tabular}

Derived values of I and $\mathbf{s}$ (and obtained $\hbar$ using these values) for all the outer planets are shown in table below. 
The obtained value of $\hbar$ for Uranus shows remarkable agreement with Neptune. The $\hbar$ values for Saturn and Jupiter still agree well with Neptune's $\hbar$ (up to the second decimal), but increase in value with increase in spin radius is obvious. Likely reason for this is oscillation of spin velocity (radius) as noticed previously in quantization of gravitational momentum. Note that this is equivalent to $\hbar$ oscillation, if one is to conserve discrete quantum numbers.

However, the orbital radius oscillates too. Note that orbital velocity is almost equal to spin velocity for planets in $2 \mathrm{e}$ configuration (Jupiter and Saturn). Setting orbital velocity equal to equatorial spin velocity and decreasing spin velocity proportionally yields much better results for Jupiter:

$$
\hbar=m_{r e} r_{a} v_{t o t}=m_{r e} r_{a}\left(\frac{v_{e}}{v_{a}} v_{a}+\frac{v_{e}}{v_{a}} v_{s}\right)=1.33 * 10^{36} \mathrm{Js}
$$

$$
\mathrm{v}_{\mathrm{e}}=12571 \mathrm{~m} / \mathrm{s}
$$

and, similarly, for Saturn:

$$
\begin{array}{r}
\hbar=m_{r e} r_{a} v_{t o t} \frac{1}{\sqrt{2}}=m_{r e} r_{a}\left(\frac{v_{a}}{v_{e}} v_{a}+\frac{v_{a}}{v_{e}} v_{s}\right) \frac{1}{\sqrt{2}}=1.3372 * 10^{36} \mathrm{Js} \\
\qquad \mathrm{v}_{\mathrm{e}}=9871 \mathrm{~m} / \mathrm{s}
\end{array}
$$

These results show that constants in QM are the result of superposition of oscillating values.

One may attempt to do the same with positive charges (terrestrial planets), however, here, determination of spin radius is more challenging and spin rotation period is not primordial.

Instead of using matter velocity, better results should be obtainable using space (Keplerian) velocity at $\mathbf{R}_{\mathrm{S}}$ (which is primordial):

$$
\begin{gathered}
v_{s}=\frac{2 \pi R_{s}}{T_{s}}=\sqrt{\frac{G M}{R_{s}}} \\
\mathrm{G}=\mathrm{G}_{0}=\text { standard gravitational constant }=6.674 * 10^{-11} \mathrm{~m}^{3} / \mathrm{kgs}^{2}
\end{gathered}
$$

One possible configuration is shown in table below (with $\mathbf{I}$ and $\mathbf{s}$ of Earth/Mercury mirroring Saturn/Jupiter, Venus/Mars mirroring Uranus/Neptune, and spin velocity of Mercury set to its perihelion velocity): 


\begin{tabular}{|c|c|c|c|c|c|c|c|c|c|c|}
\hline $\mathbf{n}$ & configuration & I & s & planet & $\begin{array}{l}\text { total } \\
\text { mass } \\
\left(10^{24}\right. \\
\mathrm{kg})\end{array}$ & $\begin{array}{l}\text { orbital } \\
\text { velocity } v_{a} \\
(\mathrm{~m} / \mathrm{s})\end{array}$ & $\begin{array}{l}\text { orbital } \\
\text { radius } r_{a} \\
\left(10^{6} \mathrm{~km}\right)\end{array}$ & $\begin{array}{l}\text { spin } \\
\text { velocity } v_{s} \\
(\mathrm{~m} / \mathrm{s})\end{array}$ & $\begin{array}{l}\text { spin } \\
\text { radius } R_{s} \\
\text { (m) }\end{array}$ & $\begin{array}{l}\text { calculated } \\
\hbar \text { (Js) }\end{array}$ \\
\hline 10 & $1 e$ & 1 & $1 / 2$ & Mars & 0.642 & 24070 & 227.92 & 27650 & 56044 & $\begin{array}{l}4.3107 \text { * } \\
10^{35}\end{array}$ \\
\hline 3 & $2 \mathrm{e}$ & 1 & 0 & Earth & 5.972 & 29780 & 149.6 & 28435 & 492971 & $\begin{array}{l}4.3107 \text { * } \\
10^{35}\end{array}$ \\
\hline 3 & $1 \mathrm{e}$ & 1 & 0 & Venus & 4.868 & 35020 & 108.21 & 45462 & 157195 & $\begin{array}{l}4.3107 \text { * } \\
10^{35}\end{array}$ \\
\hline 5 & $2 \mathrm{e}$ & 0 & 1 & Mercury & 0.330 & 47360 & 57.91 & 58980 & 6333 & $\begin{array}{l}4.3107 \text { * } \\
10^{35}\end{array}$ \\
\hline
\end{tabular}

Note that roughly the same $\hbar$ for Earth can be obtained by setting $I$ to $\mathbf{1}$, $\mathbf{s}$ to $-\mathbf{1 / 2}$, and spin velocity equal to Keplerian velocity at surface.

Note that, if primordial $v_{S}$ was Keplerian, spin radius $\mathbf{R}_{\mathrm{S}}$ should correspond to a detectable discontinuity. By these results, this may be the inner inner core boundary or a dipole offset maximum.

However, proper spin radius equivalent to the spin radius of outer planets can be calculated.

From Q1.2 - Q1.5 follows that current mass of a planet is a result of conservation of momentum (and velocity) during collapse of the orbital (non-localized) maximum to a spin maximum:

$$
m_{r e} v_{s} r_{a}=m_{i m g} v_{s} r_{s}
$$

With $\mathrm{m}_{\mathrm{re}}$ equal to $7^{*} \mathbf{1 0}^{19} \mathrm{~kg}$ and with the assumption that $\mathrm{r}_{\mathrm{a}}$ is, for all terrestrial planets, equal to current orbital radius, spin radius is:

$$
r_{s}=\frac{m_{r e}}{M} r_{a}
$$

\begin{tabular}{|c|l|l|l|l|l|}
\hline$n$ & configuration & planet & total mass $M\left(10^{24} \mathrm{~kg}\right)$ & orbital radius $\mathrm{r}_{\mathrm{a}}\left(\mathbf{1 0}^{6} \mathrm{~km}\right)$ & spin radius $\mathbf{R}_{\mathrm{S}}(\mathrm{m})$ \\
\hline 10 & $1 \mathrm{e}$ & Mars & 0.642 & 227.92 & 24851090 \\
\hline 3 & $2 \mathrm{e}$ & Earth & 5.972 & 149.6 & 1753428 \\
\hline 3 & $1 \mathrm{e}$ & Venus & 4.868 & 108.21 & 1556019 \\
\hline 5 & $2 \mathrm{e}$ & Mercury & 0.330 & 57.91 & 12283939 \\
\hline
\end{tabular}

Here, spin radius should correspond to charge radius. However, obtained radii for Mercury and Mars are much 
larger then their current surface radii, indicating that either the collapse did not occur at $r_{a}$ or there were additional collapses.

Interestingly, calculated spin radius of Mars is roughly equal to radius of Neptune. It is also roughly equal to orbital radius of Deimos, the outermost moon of Mars, which may be interpreted as evidence of Mars' primordial (ground state) charge radius and a source of quantization of Moon radii.

If that is indeed the case, remains of moon charges of Mercury might also be present around the $12 \mathrm{k}$ $\mathrm{km}$ orbit and small deviation between the obtained spin radius and the orbit of Deimos may be attributed to oscillation of radii or mass (real mass of $6.6 * 10^{19} \mathrm{~kg}$ gives the orbit of Deimos).

I believe current moons of Mars are remains of larger moons the gravitational maximums of which have collapsed into Mars in the process of planetary neurogenesis (hypothesis which will be presented later), thus, it is possible the original orbit was equal to obtained spin radius.

Collapse of moons in this process is simultaneous with the recession of the magnetic field. Moons with a distinct gravitational maximum are thus entangled with the magnetic dipole of the planet. Considering orbital period of Deimos is $\mathbf{3 0 h}$, only a couple of hours larger than the rotation period of Mars, the original Moon might have even had a direct role in the production of Mars' magnetic field.

If the obtained spin radius is the ground state radius, the excited radius [for terrestrial planets] is likely the ground radius divided by $\mathbf{n}$.

For Mercury and Mars, this gives roughly the radius of Mercury (2x radius of Mars' core, $2 x$ radius of Earth/Mercury inner core).

For Earth, this gives the inner inner core radius or possibly dipole offset maximum (the dipole offset orbital [radius] thus being the real charge radius, opposed to the induced one in the outer core).

Note that, core differentiation into molten outer and solid inner part should be associated with $2 e$ configuration. Both Mercury and Earth are hypothesized to be in $2 \mathrm{e}$ configuration and both presently do have differentiated cores. Current data on Mars indicates its core is likely entirely liquid, again, consistent with hypothesized 1e configuration. The same should thus be true for Venus.

However, even in 1e configuration, core splitting is expected in the early stage of planet development and may even periodically occur in adult form.

If Solar System maximums are oscillating between ${ }^{10} \mathrm{C}$ and ${ }^{10} \mathrm{Be}$ configuration, even with a change in scale [of a maximum], Mars must periodically exist in $2 \mathrm{e}$ configuration (acquiring one of Earth's maximums, while Earth acquires Venus' maximum). 
Even if lifetime of a ${ }^{10} \mathrm{Be}$ configuration may be short, created temperature difference in the core should be sustainable over longer periods of time if the collapse of $2 \mathrm{e}$ configuration induces splitting of a $1 \mathrm{e}$ maximum into 2 quanta.

Since both Mars and Venus appear to have been habitable on surface some time ago, both must have been in such configuration. The switch likely occurs with the end of a 1st order Solar System cycle (4.25 Gy), but it likely also has precursors of shorter duration with the end of 2nd ( $26 \mathrm{My}$ ) and 3rd (1.512 My) order cycles.

There is a high possibility that Mars' surface becomes habitable for short period of time with each end of these cycles, not only when they are synchronized with the end of the major (1st order) cycle.

In any case, as I am convinced the Solar System is at the end of a major cycle, I believe the magnetic field of Mars will be restored within a decade or a couple of decades at most, and, once it is stabilized, should persist for millions of years.

If 1 e configuration can split into a $2 \mathrm{e}$ equivalent, two maximums of $2 \mathrm{e}$ configuration can certainly fuse into a 1e equivalent maximum. Generally, this happens when a planet reaches the adult stage (acquired real mass with its own gravity stimulates inflation of the inner core maximum), but the same effect can also be achieved with suspended animation (spin momentum), as demonstrated by Mercury.

Simultaneously with increasing habitability of Mars, one can thus expect decreasing habitability of Earth.

\subsection{Quantization of radii and gravity}

From:

$$
\frac{1}{g} v r=n h_{g}
$$

and:

$$
v^{2}=r g_{s}=r \frac{G M_{\odot}}{r^{2}}=\frac{G M_{\odot}}{r}
$$

follows: 


$$
r=n^{2} \frac{g^{2} h_{g}^{2}}{G M_{\odot}}=n^{2} \frac{G M^{2} h_{g}^{2}}{r_{s}{ }^{4} M_{\odot}}
$$

While, from Q2.1 and Q2.2, orbital radius is:

$$
\begin{gathered}
r=\frac{1}{m_{r e} v_{t o t}}(\sqrt{l(l+1)}+s) \hbar=\frac{1}{m_{r e}\left(\sqrt{\frac{G M_{\odot}}{r}}+\sqrt{\frac{G k M_{\odot}}{r}}\right)}(\sqrt{l(l+1)}+s) \hbar \\
r=\frac{1}{m_{r e}{ }^{2} G M_{\odot}(1+\sqrt{k})^{2}}(\sqrt{l(l+1)}+s)^{2} \hbar^{2}
\end{gathered}
$$

For outer planets:

$$
\hbar=g_{0} h_{g} m_{r e}=1.3310 * 10^{36} \mathrm{Js}
$$

\begin{tabular}{|c|c|c|c|c|c|}
\hline n & configuration & I & $\mathbf{s}$ & planet & $\sqrt{ } \mathbf{k}$ \\
\hline 5 & $1 \mathrm{e}$ & 1 & $1 / 2$ & Neptune & $\frac{1}{2}$ \\
\hline 5 & $1 \mathrm{e}$ & 1 & 0 & Uranus & $\sqrt{\frac{1}{2}\left(\frac{1}{2}+1\right)}-\frac{1}{2}=\frac{\sqrt{3}}{2}-\frac{1}{2}$ \\
\hline 3 & $2 e$ & 1 & 0 & Saturn & 1 \\
\hline 1 & $2 e$ & 0 & 1 & Jupiter & $\sqrt{\frac{1}{2}\left(\frac{1}{2}+1\right)}=\frac{\sqrt{3}}{2}$ \\
\hline
\end{tabular}

Here, square root of $\mathbf{k}$ is another quantum momentum magnitude [sum], shown in table below:

From Q2.3 and Q2.4 follows that surface gravity is quantized:

$$
g=\frac{1}{n} \frac{(\sqrt{l(l+1)}+s)}{(1+\sqrt{k})} g_{0}
$$

where go, equal to $\mathbf{4 3 . 4 3} \mathrm{m} / \mathrm{s}^{2}$, is the quantum of gravity.

From Q2.3 and with total mass equal to: 


$$
M=m_{i m g}=m_{r e} \frac{r_{a}}{r_{s}}=m_{r e} \frac{r}{r_{s}}
$$

follows that spin radius $\mathbf{r}_{\mathbf{s}}$ is quantized too:

$$
r_{s}^{6}=n^{2} r \frac{G m_{r e}{ }^{2} h_{g}^{2}}{M_{\odot}}
$$

Combined with Q2.4 and Q2.5:

$$
\begin{gathered}
r_{s}^{3}=n \frac{(\sqrt{l(l+1)}+s)}{(1+\sqrt{k})} \frac{\hbar^{2}}{g_{0} m_{r e} M_{\odot}} \\
r_{s}=\left[n \frac{(\sqrt{l(l+1)}+s)}{(1+\sqrt{k})} \frac{g_{0} m_{r e} h_{g}^{2}}{M_{\odot}}\right]^{\frac{1}{3}}
\end{gathered}
$$

Note that the constant on the right is, for $m_{r e}=7 * 10^{19} \mathrm{~kg}$, equal to:

$$
\frac{g_{0} m_{r e} h_{g}{ }^{2}}{M_{\odot}}=2.93050621 * 10^{20} \mathrm{~m}^{3}
$$

apparently an integer multiple of the speed of light on $U_{1}$ scale $\left(2.93^{*} 10^{6} \mathrm{~m} / \mathrm{s}\right)$.

For Neptune this gives spin (charge) radius equal to half of the current surface radius - as expected, like in case of Earth, real charge radius should be half of the mass radius of the maximum (for Earth, mass radius of the maximum is the inner core radius).

Note that dipole offset for Neptune is roughly half the radius, consistent with the result.

For $m_{\text {re }}$ equal to 5.6 * $10^{20} \mathrm{~kg}$, one gets the mass radius of the maximum (surface radius).

The result is similar for Uranus.

Note that the equation might not give accurate current spin radius for Jupiter and Saturn. Reason for this may be that the initial assumption of current radius being equal to collapse radius $\left(r_{a}=r\right)$ is not valid, however, more likely explanation is oscillation of mass (and therefor, spin radius) - even if the Solar System is carbon-like, its negative and positive charge components are not necessarily all electrons and positrons. 
Inflation of mass can be asymmetric due to lepton oscillation.

However, the result for Jupiter gives radius exactly two times the dipole offset of Saturn in surface radius relative units $(2 \times 0.03778 R=0.07557 R)$, but roughly $2 / 3$ the actual dipole offset of Jupiter $(0.119$ $\mathrm{R})$. The value is also equal to dipole offset of Earth $(0.076 \mathrm{R})$.

On the other hand, the result for Saturn gives radius $0.146 \mathrm{R}(4 \times 0.0365 \mathrm{R})$, closer to dipole offset of Jupiter.

Again, these results suggest the cause for discrepancy is oscillation.

For inner planets, the constants are different:

$$
\begin{gathered}
h_{g}=h_{g_{1}}=1.482496 * 10^{14} \mathrm{~ms} \\
\hbar=g_{0} h_{g} m_{r e}=4.5069360896 * 10^{35} \mathrm{Js}
\end{gathered}
$$

\begin{tabular}{|c|c|c|c|c|c|c|}
\hline $\mathbf{n}$ & configuration & I & $\mathbf{s}$ & planet & $\sqrt{ } \mathbf{k}$ & spin radius $(\mathrm{m})$ \\
\hline 10 & $1 e$ & 1 & $1 / 2$ & Mars & $\sqrt{\frac{1}{2}\left(\frac{1}{2}+1\right)}+\frac{1}{2}=\frac{\sqrt{3}}{2}+\frac{1}{2}$ & 6477988 \\
\hline 3 & $2 e$ & 1 & 0 & Earth & 1 & 4146215 \\
\hline 3 & $1 e$ & 1 & 0 & Venus & $\sqrt{\frac{1}{2}\left(\frac{1}{2}+1\right)}+\frac{1}{2}=\frac{\sqrt{3}}{2}+\frac{1}{2}$ & 3920325 \\
\hline 5 & $2 \mathrm{e}$ & 0 & 1 & Mercury & $\sqrt{\frac{1}{2}\left(\frac{1}{2}+1\right)}+\frac{1}{2}=\frac{\sqrt{3}}{2}+\frac{1}{2}$ & 4140950 \\
\hline
\end{tabular}

and possible quantization parameters, along with the calculated spin radius, are shown in table below:

Note that the above parameters for Mars' orbital radius give a perihelion rather than a semi-major axis, suggesting that it (and generally, planets with large eccentricity) may be in a superposition of two quantum states.

Results for spin radius are obviously wrong, most likely reason for this is the bad $\mathbf{h}_{\mathbf{g}}$ constant as it is based on gravity at surface radius, which, for inner planets, is not defined as the radius of a gravitational 
maximum.

However, correlation with dipole offsets is still present. Calculated spin radius of Earth/Mercury is almost exactly 10 times the experimentally obtained dipole offset of Mercury $(\mathbf{4 1 4 . 7} \mathrm{km})$.

If the assumption of charge radius being 10 times lower than calculated spin radius for terrestrial planets is valid, somewhat larger current offset for Earth $(\mathbf{4 8 4 . 7} \mathrm{km}$ from center) must be the result of oscillation (superposition) and faster rotation.

Consistent correlation of results with dipole offsets suggests the primary or primordial source of magnetic dipoles in planets is concentrated (collapsed) orbiting charge with a large spin momentum close to the dipole offset radius, rather than currents induced with Coriolis force in outer parts of differentiated cores.

In fact, deviation of a dipole offset from calculated value should, in some part, be due to induced currents rather than oscillation.

In that case, faster rotation rates and greater liquid mass would introduce greater deviation. This is consistent with obtained results, as Jupiter and Saturn do rotate much faster than Uranus and Neptune, while Earth rotates much faster than Mercury.

However, as calculated and experimentally obtained dipole offsets both seem to be multiples of $\approx \mathbf{0 . 0 3 4 - 0 . 0 3 8 ~ R}$, only deviation from integer multiples of that quantum may be attributed to induced currents, the rest is more likely due to [quantized] oscillation.

Using the radius of a gravitational maximum for Earth $(1206115 \mathrm{~m})$, one obtains the proper $\mathbf{h}_{\mathbf{g}}$ constant for charge radius calculation of inner planets:

$$
\begin{array}{rl}
h_{g}=h_{g_{1}}=\frac{v r}{g n}=5.419815085 * 10^{12} & m s \\
& v=\text { Earth's orbital velocity }=29780 \mathrm{~m} / \mathrm{s} \\
r & =\text { Earth's orbital radius }=149.6 * 10^{9} \mathrm{~m} \\
g & =\text { gravity of the maximum }=274 \mathrm{~m} / \mathrm{s}^{2} \\
\mathrm{n}=3
\end{array}
$$

Results obtained using this constant are shown in table below: 
\begin{tabular}{l|l|l|l|l}
10 & $1 e$ & 1 & $1 / 2$ & Mars
\end{tabular}

$32 e$

\begin{tabular}{l|l|l}
1 & 0 & Earth
\end{tabular}

1

456716

$31 e$

\begin{tabular}{l|l|l}
1 & 0 & Venus
\end{tabular}

$\sqrt{\frac{1}{2}\left(\frac{1}{2}+1\right)}+\frac{1}{2}=\frac{\sqrt{3}}{2}+\frac{1}{2}$

431833

\begin{tabular}{l|l|l|l|l}
5 & $2 e$ & 0 & 1 & Mercury
\end{tabular}

$$
\sqrt{\frac{1}{2}\left(\frac{1}{2}+1\right)}+\frac{1}{2}=\frac{\sqrt{3}}{2}+\frac{1}{2}
$$

456136

These are now much closer to dipole offsets. Difference should be attributed to oscillation.

Models of the dipole location of Earth indeed show oscillation, in the last $\mathbf{1 0 0 0 0}$ years it has oscillated from a maximum of $\mathbf{4 1 4 . 7} \mathrm{km}$ (equal to a dipole offset of Mercury) in the western hemisphere to a maximum of $\mathbf{5 5 4 . 7} \mathbf{k m}$ in the eastern hemisphere ${ }^{16}$.

Dipole offset in current models is thus a superposition (arithmetic mean) of these two maximums (484.7 $\mathrm{km})$.

The agreement of $\mathbf{4 1 4 . 7} \mathrm{km}$ maximum with the dipole offset of Mercury suggests that either:

- the influence of rotation on the offset is negligible,

- rotation stops once the maximum is reached,

- induced currents are created at the expense of primary charge, effectively transferring the charge radius from inner core to outer core.

Possibly, this is the effect of conservation of momentum, where spin of the primary charge is reduced at the expense of core rotation.

\subsection{Lepton oscillation model}

In the previous chapter it was hypothesized that the discrepancy between the QM model of the atom and the Solar System can be resolved by lepton oscillation.

This can be solely mass oscillation, which requires external energy, or the oscillation of general force flavor which does not require external energy as mass is inflated with the exchange of polarized (electro- 
magnetic) potential with a neutral gravitational potential (it does need stimulation though, most likely by resonance - synchronization).

However, while general force flavor has certainly been changed to [dominantly] neutral with a change in vertical energy level, difference in mass between the outer planets is too large compared to a difference in electro-magnetic energy to be explained by general force oscillation alone.

If the Solar System has been inflated, as hypothesized, from a smaller scale atom, then likely there was enough energy for a superposition of electron mass eigenstates.

Taking into account that these electrons are also neutralized, superposition becomes even more likely (charged leptons repel) due to lower energy requirements.

With oscillation and inflation taken into account, the fact that planets of the Solar System have different masses goes in favor of it being the atom, rather than against it.

However, the excess energy left after the vertical energy level increase (inflation) might not be the only source of superposition. Most energy in the vertical energy level change is spent on inflation - not flavor oscillation, so even without inflation, the flavor oscillation energy can be provided by the nucleus or absorption of properly scaled gravitational waves.

Atoms which are not under influence of strong external magnetic fields may be dominantly in neutral or oscillating configuration, regardless of scale - there is a lot of energy for mass oscillation in nucleons.

If neutrinos oscillate in flight they must be absorbing energy in space, but their flavor may instead be predetermined with oscillation of particles inside the atom. In fact, lepton oscillation might be confined to atoms if discharge of outermost particles is synchronized with their mass flavor being in ground (lowest energy) state in a form of electron.

There is no such requirement for neutrinos as their mass is much lower than that of the electron. Probability of discharge of masses greater than electron mass might be simply too low due to much greater gravitational attraction.

With no absolute constants allowed and implied oscillation of relative constants, oscillation in the energy of space is predicted by $\mathbf{C R}$.

With no oscillation, in the Solar System, the inner planets would all be in positron equivalent states, while outer planets would be scaled electrons. 
Note that, with em force almost completely neutralized (especially for inner planets), due to equal energy of positrons and electrons there are no large differences between these particles, apart from antialignment of magnetic spins.

One might ask why and how are positrons created (extracted) here? The answer is in neutralization when charged they balance the electrons and, most likely, they are, together with neutrinos (main dwarf planets), the result of $\beta^{+}$decay of protons. However, due to charge neutralization, instead of being ejected from the nucleus, they remain bound to it.

The $\beta^{+}$process implies that each positron $\left(1 \mathrm{e}^{+}\right.$terrestrial planet) or positron pair $\left(2 \mathrm{e}^{+}\right.$terrestrial planet) is entangled with a specific neutrino ( $1 v_{e}$ main dwarf planet) or a neutrino pair $\left(2 v_{e}\right.$ main dwarf planet) since the entangled pairs have been created at the same time, through the intermediate $\mathbf{W}^{+}$boson.

Note also, that, in order for the equation Q1.1 to remain valid, the excitation of Neptune must be equal to the [scaled] excitation of the nucleus (Sun).

Thus, for the Solar System atom, and perhaps generally, the oscillation is synchronized between the innermost and outermost parts of the atom, consistent with absorption of wave-like forms of energy.

The oscillation can thus explain the difference in masses between the planets, but the oscillation itself should be quantized.

\subsubsection{The creation}

Applying neutralization and lepton oscillation to the model of inflation (vertical energy level change), one can now reconstruct the history of the Solar System development.

With inflation, the [absolute] distance between particles is increasing. Assuming the system started in polarized state, the neutralization will be decreasing [relative] distance between equally charged particles.

Note 1: The only reason for neutralization during inflation may be a difference in [relative] external magnetic field strengths between the two scales, with larger scale system being under influence of much weaker magnetic fields.

Note 2: If the inflation starts with an already neutral system, the end result is similar. In that case, large scale system is a relative clone of the small scale system, with no energy wasted on neutralization, only on inflation. However, any excess energy (beyond the discrete energy needed to change the vertical energy level) will result in cloning imperfections proportional to the excess energy. 
Note 3: Only naked [gravitational] maximums are inflated, real mass is acquired during and after inflation from existing mass (asteroid) fields. These fields are generally created with deflation of other maximums in nova like explosions. Deaths (deflations) and births (inflations) of a particular scale are relatively synchronized.

Based on wave-like appliance of energy, the inflation may have proceeded in this order:

1. Nucleus started inflating.

2. Jupiter $2 \mathrm{e}$ configuration started inflating. Even though $2 \mathrm{e}$ may have been separated initially, large energy of this configuration enabled the fusion of 2 electrons. With the inflation of Jupiter, $2 \mathrm{e}$ positron configuration was inflating. However, this configuration did not have enough energy for fusion and the positrons were left separated enough to form Mars $\left(1 \mathrm{e}^{+}\right)$and Vulcan (1 $\mathrm{e}^{+}$, Mercury embryo).

3. Saturn $2 e$ configuration started inflating. This one had less energy that Jupiter $2 e$, but still enough for fusion, while the positrons again, did not - however, the energy was bigger than in the first positron pair, resulting in the creation of Venus $\left(1 \mathrm{e}^{+}\right)$and Gaia $\left(1 \mathrm{e}^{+}\right.$, Earth embryo).

4. Another $2 \mathrm{e}$ configuration started inflating. This one had even less energy than Saturn $2 e$, and, this time, not enough for fusion, so $2 \mathrm{e}$ separated into Uranus (1e) and Neptune (1e). A [relatively] simultaneous $2 \mathrm{e}^{+}$inflation resulted in fusion of $1 \mathrm{e}^{+}$with Vulcan, creating Mercury, and fusion of the other $1 \mathrm{e}^{+}$with Gaia, creating Earth.

Note that, on the right (outer) side, the energy of inflation is decreasing, while on the left (inner) it is increasing. This fluctuation is the result of an attempt to balance the left and right side of the system.

Note also, that, if the original (small scale) system was in an electric field, the system did have a left and right (or top and bottom) side, not only inner and outer orbits.

Comparing the energies of the planets, the lepton oscillation and the [attempt of] energy balancing is obvious.

Assuming that scaled mass of a standard electron $\left(0.511 \mathrm{MeV} / \mathrm{c}^{2}\right)$ is equal to $0.511 * 10^{24} \mathrm{~kg}$, scaled muon $\left(105.658 \mathrm{MeV} / \mathrm{c}^{2}\right)$ is $105.658 * 10^{24} \mathrm{~kg}$, while scaled tau particle $\left(\mathbf{1 7 7 6 . 8 6} \mathrm{MeV} / \mathrm{c}^{2}\right)$ has a mass of $1776.86 * 10^{24} \mathrm{~kg}$, rough correlation with masses of Mercury/Mars, Neptune/Uranus and Jupiter is obvious.

The tau/muon/electron mass ratios are present within the inner and outer planets:

$$
\frac{\text { Venus }+ \text { Earth }}{\text { Mars }} \approx \frac{\text { Venus }+ \text { Earth }}{2 \text { Mercury }} \approx \frac{\text { tau }}{\text { muon }}
$$




$$
\begin{gathered}
\frac{\text { Neptune }}{\text { Earth }} \approx \frac{\text { Uranus }}{\text { Venus }} \approx \frac{\text { Jupiter }}{\text { Neptune }} \approx \frac{\text { tau }}{\text { muon }} \\
\frac{\text { Outer planets }}{\text { Inner planets }} \approx \frac{\text { Uranus }+ \text { Neptune }}{\text { Mercury embryo+Mars }} \approx \frac{\text { muon }}{\text { electron }}
\end{gathered}
$$

but also in relation to the Sun:

$$
\frac{\text { Sun }}{\text { Saturn }} \approx \frac{\operatorname{tau}}{\text { electron }}
$$

which suggests that the whole system is in superposition of particles of different generations.

The grouping and correlation of Venus/Earth and Uranus/Neptune here is understandable, as the pairs share the same quantum shell.

Correlation of Uranus/Neptune with Mercury/Mars lies in the fact that Mars and Mercury [embryo] were the first pair created on the inner side, while Uranus and Neptune were the last to be created on the outer side - with increasing energy on the inner side and decreasing on the outer side, the ratio of Uranus+Neptune/Mercury+Mars becomes roughly equal to the ratio of mass of outer to inner planets. This gives mass of $0.198 * 10^{24} \mathrm{~kg}$ for the Mercury embryo $\left(1 e^{+}\right)$. Comparing Venus $\left(1 e^{+}\right)$and Earth $\left(2 e^{+}\right)$, the addition of another maximum doesn't impact the total mass significantly (as most energy comes from neutralization which is, at least roughly, invariant to number of particles occupying the state).

If the Mercury embryo mass was core mass, total core mass of current Mercury should be equal to:

$$
\frac{\text { Earth core }}{\text { Venus core }}(\text { Mercury embryo mass })=\frac{0.325}{0.32} 0.198 * 10^{24} \mathrm{~kg}=0.2011 * 10^{24} \mathrm{~kg}
$$

$61 \%$ of its total mass (for Venus' core at $32 \%$ of total mass, Earth's core at $\mathbf{3 2 . 5} \%$ of total mass).

In Fig. 1 below, standard particle candidates are shown for each planet. Rest masses are relative to the possible event horizon of creation, specified in parentheses. Note that original rest mass may be bigger or smaller than relativistic mass, depending on the conditions in the annihilation (creation) event.

Most likely particle candidates are marked green.

planet

relativistic mass $M\left[10^{24} \mathrm{~kg}\right]$

(v) rest mass $M_{0}$ candidates $\left[10^{24} \mathrm{~kg}\right]$ (CEH) particle candidates $\left(\mathrm{MeV} / \mathrm{c}^{2}\right)$ 


\begin{tabular}{|c|c|c|c|}
\hline Venus & $4.868(35.0 \mathrm{~km} / \mathrm{s})$ & $\begin{array}{l}5.67(17.905 \mathrm{~km} / \mathrm{s}=\text { Ceres orbit }) \\
5.545(16.76 \mathrm{~km} / \mathrm{s}=\text { Hygiea orbit })\end{array}$ & down anti-quark $(\approx \mathbf{4 . 8})$ \\
\hline Earth & $5.972(29.8 \mathrm{~km} / \mathrm{s})$ & $\begin{array}{l}7.47(17.905 \mathrm{~km} / \mathrm{s}=\text { Ceres orbit }) \\
7.47(17.89 \mathrm{~km} / \mathrm{s}=\text { Pallas orbit }) \\
4.77(-17.905 \mathrm{~km} / \mathrm{s}=- \text { Ceres orbit })\end{array}$ & down anti-quark $(\approx \mathbf{4 . 8})$ \\
\hline Mars & $0.642(24.1 \mathrm{~km} / \mathrm{s})$ & $\begin{array}{l}1.076(19.34 \mathrm{~km} / \mathrm{s}=\text { Vesta orbit }) \\
0.383(-19.34 \mathrm{~km} / \mathrm{s}=- \text { Vesta orbit }) \\
0.461(-16.76 \mathrm{~km} / \mathrm{s}=- \text { Hygiea orbit }) \\
0.539(-13.1 \mathrm{~km} / \mathrm{s}=- \text { Jupiter orbit })\end{array}$ & positron $(\mathbf{0 . 5 1 1})$ \\
\hline Jupiter & $1898.19(13.1 \mathrm{~km} / \mathrm{s})$ & $\begin{array}{l}1396(-19.34 \mathrm{~km} / \mathrm{s}=- \text { Vesta orbit }) \\
1293(-17.905 \mathrm{~km} / \mathrm{s}=- \text {-Ceres orbit), } \\
1824(-47.4 \mathrm{~km} / \mathrm{s}=- \text { Mercury orbit })\end{array}$ & $\begin{array}{l}D^{-} \text {meson (1869), tau (1776.86), charm anti-quark } \\
(\approx 1275)\end{array}$ \\
\hline Saturn & $568.34(9.7 \mathrm{~km} / \mathrm{s})$ & $\begin{array}{l}491.4(-19.34 \mathrm{~km} / \mathrm{s}=- \text { Vesta orbit }) \\
477.7(-17.905 \mathrm{~km} / \mathrm{s}=- \text { Ceres orbit })\end{array}$ & $\mathrm{K}^{-}$meson (493.7) \\
\hline Uranus & $86.813(6.8 \mathrm{~km} / \mathrm{s})$ & $\begin{array}{l}80.285(-17.89 \mathrm{~km} / \mathrm{s}=- \text { Pallas orbit }) \\
94.982(16.76 \mathrm{~km} / \mathrm{s}=\text { Hygiea orbit })\end{array}$ & muon (105.658), strange quark $(\approx 95)$ \\
\hline Neptune & $102.413(5.43 \mathrm{~km} / \mathrm{s})$ & $96.5(-16.76 \mathrm{~km} / \mathrm{s}=-$ Hygiea orbit $)$ & muon (105.658), strange quark ( $\approx 95)$ \\
\hline
\end{tabular}

Fig. 1: Standard particle candidates for planets (green = most likely)

Rest mass in Fig. 1 was calculated using proper relativistic Lorentz factor (Omega factor):

$$
\begin{gathered}
M_{0}=M\left[1-\left(\frac{v^{2}}{c_{n}{ }^{2}}\right)^{s}\right]^{-\frac{1}{2} q} \\
q=\operatorname{sgn}\left(c_{n}\right)=\frac{c_{n}}{\left|c_{n}\right|} \\
s=\operatorname{sgn}\left(c_{n}{ }^{2}-v^{2}\right)=\frac{c_{n}{ }^{2}-v^{2}}{\left|c_{n}{ }^{2}-v^{2}\right|} \\
\quad \mathrm{q}=\text { mass charge of the reference frame } \\
\mathrm{s}=\mathbf{0} \text { spin charge of the reference frame }
\end{gathered}
$$

where $c_{n}=C_{E H}$ is the rest velocity of the reference frame (event horizon [fossil]).

Note 1: The correlation suggests that inflation energy for these planets came individually from specific particles, with roughly equal kinetic energy. This is consistent with the hypothesized matter/anti-matter atom pair annihilation - with colliding positron/electron pairs producing the particles inflated into planets.

Such annihilation would likely occur within the gravitational maximum (event horizon) discontinuity, sending created matter and anti-matter in opposite directions, perpendicular to the maximum. 
The central galactic black holes and dark matter maximums in inner/outer layers of galaxies are likely remnants of such maximums.

Note 2: Due to neutralization, there are no significant differences between planetary systems created from matter and anti-matter atoms, apart from mass distribution - if the Solar System is created from matter (a matter of convention), in an anti Solar System inner planets would have greater mass than outer planets. The reason for this is the asymmetry of space at the event horizon, where opposite charges are separated to opposite sides of the horizon. Note that this implies that horizons are, at the moment of collapse, between outer and inner planets where, after collapse, a neutrino (main dwarf planet) is formed. Note that creation of matter at event horizons resolves the missing anti-matter problem in physics - there is no anti-matter missing, there is asymmetry in mass acquisition of stable charges due to non-homogeneous energy of space, proton is anti-matter equivalent of the electron from a proper reference frame.

Thus, all positively charged particles are anti-matter particles, while negatively charged particles are matter particles (or vice versa, in alternative convention).

Note also that an event horizon for electron/positron annihilation can be provided by the atom nucleus itself - with the incoming electron, a maximum is extracted (expanded) from the nucleus (and possibly from the electron too) together with positron charge. At the point of annihilation, maximum (or a maximum pair) collapses with energy distributed between the created neutrino(s) and two charged particles, with none of them having enough energy to overcome escape velocity.

Due to mass asymmetry the pair will not annihilate again and the external energy (photons) is required to decouple mass and charge, and return the system to original state.

Likely, all annihilation events require expansion of particle maximums and creation of a temporary event horizon pair even if one [of larger scale] is already present at the point of annihilation.

Note 3: In CR, not only the flavors are oscillating, but, neither the rest or inertial mass is constant. Deviation from average mass is greatest in bound systems where it depends on the energy level particle occupies in the system.

Note 4: Correlation of standard masses with planetary rest mass in reference frames of orbits of bound neutrinos (main dwarf planets) is overall very good, with lower confidence only in case of the participants of the first planetary creation event - Mars and Mercury (unless the standard particle equivalent is yet to be discovered).

For Mars, the horizon at Jupiter orbit is a better fit, while for Mercury, it is the Venus orbit.

Note 5: Correlation of the Solar System with standard scale particle generations, reveals the existence of new particles in the standard model of physics (which, obviously, should be scale invariant), for example, if 
; one interprets Saturn as $\mathbf{K}^{-}$, the Sun/Saturn mass equivalence with tau/electron reveals 2 additional standard particles:

$$
\begin{gathered}
\frac{\text { tau }}{\text { electron }} K^{-}=1717.751 \mathrm{GeV}=1.72 \mathrm{TeV} \\
\frac{\text { muon }}{\text { electron }} K^{-}=102.143 \mathrm{GeV}
\end{gathered}
$$

or, with the assumption of new energy splitting, a completely new generation (based on Sun's relativistic mass):

or, with Sun's proper rest mass:

$$
\begin{gathered}
\frac{\text { tau }}{\text { electron }} X^{n}=3477.228 * 571.864 \mathrm{MeV}=1988.500 \mathrm{GeV}=1.9885 \mathrm{TeV} \\
\frac{\text { muon }}{\text { electron }} X^{n}=206.768 * 571.864=118.243 \mathrm{GeV}
\end{gathered}
$$

$$
\begin{gathered}
\frac{\operatorname{tau}}{\text { electron }} X^{n}=3477.228 * 537.552 \mathrm{MeV}=1869.190 \mathrm{GeV}=1.8692 \mathrm{TeV} \\
\frac{\text { muon }}{\text { electron }} X^{n}=206.768 * 537.552=111.149 \mathrm{GeV}
\end{gathered}
$$

\begin{tabular}{|c|c|c|c|}
\hline planet & configuration & particle species (charge) & total charge \\
\hline Mercury & $2 \mathrm{e}$ & positron $\left(1 \mathrm{e}^{+}\right)$ & $2 e^{+}$ \\
\hline Venus & $1 e$ & down anti-quark $\left(1 / 3 \mathrm{e}^{+}\right)$ & $1 / 3 e^{+}$ \\
\hline Earth & $2 \mathrm{e}$ & down anti-quark $\left(1 / 3 \mathrm{e}^{+}\right)$ & $2 / 3 \mathrm{e}^{+}$ \\
\hline Mars & $1 e$ & positron $\left(1 \mathrm{e}^{+}\right)$ & $1 \mathrm{e}^{+}$ \\
\hline Jupiter & $2 \mathrm{e}$ & charm anti-quark $\left(2 / 3 \mathrm{e}^{-}\right)$ & $4 / 3 e^{-}$ \\
\hline Saturn & $2 \mathrm{e}$ & $\mathbf{K}^{-}$meson $\left(1 \mathrm{e}^{-}\right)$ & $2 e^{-}$ \\
\hline Uranus & $1 e$ & strange quark $\left(1 / 3 \mathrm{e}^{-}\right)$ & $1 / 3 e^{-}$ \\
\hline Neptune & $1 e$ & strange quark $\left(1 / 3 \mathrm{e}^{-}\right)$ & $1 / 3 e^{-}$ \\
\hline
\end{tabular}

One of these may have been discovered ${ }^{17}$ already $^{18}$.

Evidently, using most likely particle candidates on the hypothesized particle configuration, the electric charges are in balance, as shown on Fig. 2.

Fig. 2: Standard particle candidates for planets with listed electric charges

The configuration gives total $4 \mathrm{e}^{+}$charge for inner planets and $4 \mathrm{e}^{-}$for outer planets. 
The fact that charge configuration agrees well with the hypothesis of $\mathbf{6}$ particles on each side (Carbon configuration) but the mass for the same particle species agrees well with 4 total particles on each side (Beryllium configuration) indicates that the original hypothesis of ${ }^{10} \mathrm{C} /{ }^{10} \mathrm{Be}$ oscillation is correct.

The fact that the sum of charges on each sides is equal to 4 , further confirms the hypothesis.

Obviously, the Solar System is a hybrid, a superposition of 2 large scale atoms, ${ }^{10} \mathrm{C}$ and ${ }^{10} \mathrm{Be}$.

Is this hybridization unique to the inflation through annihilation of smaller scale atoms, or this is a normal state even in atoms of standard scale?

In $\mathbf{C R}$, of course, the process is scale invariant and cannot be unique to one scale only, even if one cannot set up a proper reference frame to observe it.

The stability of atoms is achieved through neutral energy provided by neutral cores.

It is thus likely that all atoms are oscillating between polarized and non-polarized states.

Consider the case of elementary hydrogen $\left({ }^{1} \mathbf{H}\right)$.

If 1 e+ charge (ie. positron) is extracted from the nucleus to balance the electron, what prevents them from annihilating?

Obviously, between the two particles there must exist an event horizon [pair], which collapses in the interaction, forming a [bound] neutrino, but also emitting a gravitational wave of 2 maximum quanta, one of which is absorbed by the electron, the other by the positron - pushing them to stable orbits and preventing annihilation.

Note that both positron and electron are now [even more] entangled and form a standing wave.

If absorbed maximums are neutral they will increase masses of particles, decreasing charges (albeit in asymmetric manner relative to event horizon). This may be negligible but a probability exists the absorption will trigger charge [scale] collapse and mass [scale] inflation inverting the dominant nature of general force (em/gravity exchange) between the particles.

Note that, with charge extracted, proton core too becomes neutral.

It appears that [outer event horizons of] proton cores favor giving energy to electrons, while neutrons 
favor positrons (correlated with spin anti-alignment). Asymmetry in neutralization energy between bound positrons and electrons is thus caused in mass difference between protons and neutrons (note that magnetic fields of outer planets are much less subdued than those of inner planets).

If one interprets Neptune as the electron, Jupiter contains the mass of two down quarks, while Saturn mass has been increased with the equivalent of one up quark mass (note that charges were separated from mass before neutralization).

Assuming these masses came from protons (nucleus is scaled equally to Neptune), there are only 4 complete protons left in the nucleus. If now free up quarks [masses] couple with down quarks of a neutron, it will be converted to 2 protons. With 6 protons and 3 neutrons left, 3 more neutrons are needed to balance the core.

With a complete neutron ( 2 down quarks +1 up quark) mass on the outer side, and with remaining proton quarks left in the core, it might seem that neutralization is quantized by neutron mass.

However, the fact that Neptune and Uranus are significantly neutralized suggests that neutralization energy is correlated with quantum states and is likely scaling with element mass.

In any case, gas planets should always be the most charged ones.

Note that, with imaginary mass being dark matter and with outer planets having significant excess of gravitational potential compared to inner planets, Solar System mirrors the galaxy.

The reason why outer planets and nearby masses are not rotating faster is due to collapse of orbital maximums into spin momenta and acquisition of real mass.

Spin coupling, in case of carbon, indicates that, as a whole system, ${ }^{12} \mathrm{C}$ is more stable than ${ }^{13} \mathrm{C}$, while ${ }^{13} \mathrm{C}$ nucleus is, due to equal number of protons and neutrons, more stable than ${ }^{12} \mathrm{C}$ nucleus.

With an excess of protons, too much energy on the outer side can cause the ejection of bound positrons and neutrinos, converting protons to neutrons.

With an excess of neutrons, too much energy on the inner side can be enough to fuse bound positrons with the nucleus [core], converting neutrons to protons.

\subsubsection{Evaluation of invariance}

Correlation between planetary masses and standard particles revealed in the previous chapter is 
remarkable, not only because ratios of particle masses are equal on both scales, but numeric values seem to be equal between kilograms on one scale and electron volts on another - differing only in the order of magnitude.

This reveals interesting relation between electric charge and speed of light:

$$
\begin{gathered}
e V=m \frac{c^{2}}{e} \\
m=e V K \\
e=c^{2} K
\end{gathered}
$$

where $\mathrm{K}$ on the solar system $\left(\mathrm{U}_{1}\right)$ scale is $1 * 10^{18} \mathrm{Cs}^{2} / \mathrm{m}^{2}$.

Since planetary mass is derived from $\mathbf{G M}$ product, integer value of $\mathbf{K}$ must be the consequence of dependence of the gravitational constant $\mathbf{G}$ on the speed of light $\mathbf{c}$.

Both values, gravitational constant $\mathbf{G}$ and $\mathbf{c}$, have been determined from standard scale $\left(\mathbf{U}_{\mathbf{0}}\right)$ experiments, thus:

$$
\begin{gathered}
G=G_{0} \\
c=c_{0}
\end{gathered}
$$

Mass $\mathbf{M}$ of the planet is then determined through gravitational interaction between two bodies, equalizing centripetal force with gravitational force:

$$
\begin{gathered}
\frac{m v^{2}}{r}=\frac{G M m}{r^{2}} \\
v^{2}=\frac{G M}{r} \\
M=\frac{v^{2} r}{G} \\
\frac{v^{2} r}{G_{0}} \frac{1}{K}=m_{0} \frac{c_{0}^{2}}{e_{0}} \\
\frac{v^{2} r}{G_{0}} \frac{c_{1}^{2}}{e_{1}}=m_{0} \frac{c_{0}^{2}}{e_{0}}
\end{gathered}
$$

where $\mathbf{r}$ is the distance [from center] to the orbiting body [center], and $\mathbf{v}$ is its orbital velocity, and, in case of planets, also the fossil of the rest velocity of the gravitational field line (orbital maximum) before the collapse 
into a spin (satellite) maximum.

Planets orbiting at rest velocity are effectively at rest in the system. Since every gravitational maximum has its personal space-time - planetary orbitals are orbits of space-time within another space-time.

Equalizing centripetal force with electro-magnetic force:

$$
\begin{gathered}
\frac{m v^{2}}{r}=\frac{1}{4 \pi \epsilon_{0}} \frac{e^{2}}{r^{2}}=\frac{\mu_{0} c^{2}}{4 \pi} \frac{e^{2}}{r^{2}}=1 * 10^{-7} c^{2} \frac{e^{2}}{r^{2}} \\
m=1 * 10^{-7} \frac{c^{2}}{v^{2}} \frac{e^{2}}{r}
\end{gathered}
$$

Now equalizing $\mathbf{M}$ (gravitational mass) and $\mathbf{m}$ (charge mass):

$$
\begin{gathered}
M=m \\
\frac{v^{2} r}{G}=1 * 10^{-7} \frac{c^{2}}{v^{2}} \frac{e^{2}}{r} \\
G=1 * 10^{7} \frac{1}{c^{2} e_{0}{ }^{2}} v_{0}{ }^{4} r_{0}{ }^{2}
\end{gathered}
$$

\section{4. $\hbar$ constant weakness}

Obvious dependency on the order of mass magnitude makes $\hbar$ a weak "constant", but at the same time explains why planetary orbits appear discrete while the orbits of small satellites seem unlimited. Obviously all masses $\mathbf{m}>\mathbf{0}$ must have a quantized momentum. 


\section{G relativity and equivalence with gravity}

If gravity is quantized and total mass $\mathbf{M}$ derived from gravity does not reveal quantization of angular momentum, apart from $\hbar$ scale dependence (oscillation), alternative interpretation is a variable gravitational constant $\mathbf{G}$.

It is then a property of a gravitational well (maximum) and it depends on its scale.

Orbital angular momentum:

$$
M v r=n \hbar
$$

multiplied with (surface) gravity is:

$$
\begin{aligned}
& g M v r=g n \hbar \\
& g=\frac{v r}{n \hbar} g M
\end{aligned}
$$

Fixing $\mathbf{g}$ on the right side (ie. $\mathbf{M}=$ mass of Neptune, $\mathbf{g}_{\mathbf{0}}=$ gravity of Neptune), multiplying with $\mathbf{R}^{\mathbf{2}} / \mathbf{R}^{\mathbf{2}}$ :

$$
g=\frac{v r}{n \hbar} g_{0} M \frac{R^{2}}{R^{2}}
$$

Fixing $\mathbf{R}$ in the numerator (ie. $\mathbf{R}_{\mathbf{0}}=$ radius of Neptune) and equalizing with Newton gravity:

$$
g=\frac{v r}{n \hbar} g_{0} R_{0}^{2} \frac{M}{R^{2}}=\frac{G M}{R^{2}}
$$

Gravitational constant is:

$$
G=\frac{v r}{n \hbar} g_{0} R_{0}^{2}
$$

$$
\begin{array}{r}
v=\text { orbital velocity } \\
r=\text { orbital radius }
\end{array}
$$

$\mathrm{R}=$ radius of the planet (spin radius)

Here, $\mathbf{v}, \mathbf{r}$ and $\mathbf{n}$ are variable. One might then consider $\hbar$ a relatively strong constant, but $\mathbf{g}_{\mathbf{0}}$ and $\mathbf{R}_{\mathbf{0}}$ are weak.

It has been shown that $g_{0}$ alternates between two values (one taking rotation into account and one without it). The following can be concluded: 
- all planets are entangled,

- past/future state of $\mathbf{g}_{\mathbf{0}} / \mathbf{R}_{\mathbf{0}}$ is fossilized/memorized in rotation period,

- planets can be entangled with a past/future state of $\mathbf{g}_{\mathbf{0}} / \mathbf{R}_{\mathbf{0}}$,

- gravitational constant $\mathbf{G}$ of a gravitational well depends on its own place in a larger gravitational well.

Note that $\mathbf{G}$ of a planetary gravitational well is here derived form its orbital momentum in a larger well, rather than its spin momentum.

One might consider planets orbiting stars, but they are also orbiting themselves (their souls). Mantle of a planet can be interpreted as expanded gravitational maximum of its moon, just like the moon can be interpreted as a collapsed gravitational maximum (event horizon) of the planet. In that system, mantle/moon is the planet and a planetary core is the star.

In case the planet is not fully developed (has active moons - in case of inner planets, or doesn't have active moons - in case of outer planets), by the same analogy, mantle layers are asteroid belts and moons are the planets of opposite charge to the outer core of the planet.

Thus, there are gravitational constants relative to that system (note that every spin momentum is orbital momentum - even though the surface and the center are entangled, propagation of changes is not instant $=$ there are no absolute point particles).

With obvious equivalence of standard quantum systems and planetary systems it would be ludicrous to insist on non-intuitive concepts of quantum mechanics and absolute constants in relativity, especially, when no non-intuitive and non-changing phenomena have ever been directly observed in reality - all are based on [non-intuitive] assumptions of existing theories.

If one is to unify forces of nature in a simple and elegant way, one should, once again, imitate nature implementing scale invariance of these forces, momentum/energy equivalence and relativity of everything, including that equivalence.

Current value of the standard gravitational constant $\left(6.674 * 10^{-11} \mathrm{~m}^{3} / \mathrm{kgs}^{2}\right)$ is tied to Earth surface, for other gravitational wells of the Solar System it can be obtained from surface gravity and real mass:

$$
g=\frac{\hbar_{m g}}{m}=\frac{G M}{R^{2}}
$$

Assuming speed of matter is significantly lower than the speed of space (generally valid): 


$$
m=\frac{2 \pi^{2} r_{0}^{3}}{G T_{1}^{2}}
$$

from (G1.1) and (G1.2) follows:

$$
\begin{gathered}
\hbar_{m g} \frac{G T_{1}^{2}}{2 \pi^{2} r_{0}^{3}}=\frac{G M}{R^{2}} \\
M=\hbar_{m g} \frac{T_{1}^{2} R^{2}}{2 \pi^{2} r_{0}^{3}}
\end{gathered}
$$

with $\mathbf{M}$ calculated, one can now obtain $\mathbf{G}$ through (G1.1):

$$
G=\frac{g R^{2}}{M}=\frac{1}{\hbar_{m g}} \frac{g 2 \pi^{2} r_{0}^{3}}{T_{1}^{2}}=\frac{1}{\hbar_{m g}} \frac{g v_{1}^{2} r_{0}}{2}=\frac{v^{4}}{\hbar_{m g}}
$$

For Earth, rotation period of the maximum (inner core) is equal to surface rotation period and $v$ is equal to surface rotation velocity.

Note that this can also be written as:

$$
\begin{gathered}
G=\frac{1}{2} \frac{v_{1} r_{0}}{\hbar_{m g}} g \frac{2 \pi r_{0}}{T_{1}} \\
G=\frac{v_{1} r_{0}}{\hbar_{m}} \hbar_{g} g \frac{\pi r_{0}}{T_{1}}=\frac{v_{1} r_{0}}{\hbar_{m}} \frac{v R}{n g} g \frac{\pi r_{0}}{T_{1}} \\
G=\frac{v_{1} r_{0}}{\hbar_{m}} \frac{\pi R^{2}}{T n} \frac{2 \pi r_{0}}{T_{1}}=\frac{v_{1} r_{0}}{n \hbar_{m}} \frac{2 \pi^{2} r_{0}}{T T_{1}} R^{2}
\end{gathered}
$$

substituting middle term for $\mathbf{g}_{\mathbf{0}}$ :

$$
\begin{aligned}
& g_{0}= \frac{2 \pi^{2} r_{0}}{T T_{1}} \\
& G= \frac{v_{1} r_{0}}{n \hbar_{m}} g_{0} R^{2} \\
& \mathrm{v}_{\mathbf{1}}=\text { matter rotation speed at the gravitational maximum } \mathrm{r}_{\mathbf{0}} \\
& \mathrm{R}=\text { planet radius }
\end{aligned}
$$

This relation is now equivalent to the obtained relation for $\mathbf{G}$ relative to orbital angular momentum.

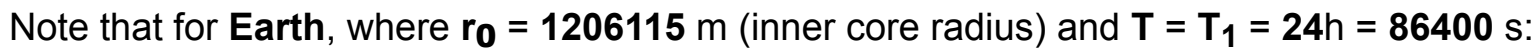




$$
g_{0}=0.00319 \frac{m}{s^{2}}
$$

which would match exactly the gravity of the inner core with virtual mass $\mathbf{M}$ equal to previously calculated real mass of Earth $\left(m=6.95 * 10^{19} \mathrm{~kg}\right)$ :

$$
g_{0}=\frac{G m}{r_{0}^{2}}=0.00319 \frac{m}{s^{2}}
$$

Since $r_{\mathbf{0}}$ and mass below it remains constant, increase of $g_{0}$ must be the result of increase in gravitational constant $\mathbf{G}$.

The current value of the gravitational constant $\mathbf{G}$ for Earth's inner core is (having $\mathbf{g}_{\mathbf{0}}=\mathbf{2 7 4} \mathrm{m} / \mathrm{s}^{2}$ ):

$$
G=\frac{g_{0} r_{0}^{2}}{m}=5.731534632 * 10^{-6} \frac{\mathrm{m}^{3}}{k g s^{2}}
$$

Gravitational constant should not differ much between terrestrial planets, thus, evidently from this, inner cores of these planets should have roughly equal period of rotation $\left(\mathbf{T}_{\mathbf{1}}\right)$ to Earth's inner core rotation period, or even shorter.

However, as stated before, rotation period for inner planets (solid bodies in general) is, generally, not primordial, and, while it gives good results for Earth (suggesting its primordial rotation period was equal to current, even though it varied over time), it is not a reliable parameter in determination of $\mathbf{G}$.

\subsection{Evidence for a constant change of $G$}

If $\mathbf{G}$ is variable, it should generally increase at the expense of the Coulomb constant, although changes in space cannot be instant and some phase shift at distance will exist.

In a bound configuration such as a Solar System, change in $\mathbf{G}$ of local space will be reflected in changes of orbital momentum.

Taking mass and distance into account, major influence on $\mathbf{G}$ on Earth is the interaction with the Sun (multiple orders of magnitude larger than the Moon and planets).

The $\mathbf{G}$ constant should thus oscillate, with the 1st order sinusoidal oscillation due to Earth's elliptical orbit of the Sun.

In the $6 \mathrm{p} 4 \mathrm{n}$ configuration of the Solar System, the existence of a perihelion and aphelion in the Earth's orbit is reflected in two discontinuities of the Sun, at 2/3 R and 1/2 R. 
With a change in distance from the Sun, spin velocity of Earth's maximum is changing relative to the rest frame of the two discontinuities - it's radius is expanding and contracting, directly affecting the local $\mathbf{G}$ constant.

Mean change of $\mathbf{G}$ due to perihelion is thus:

$$
\Delta G_{p}=\frac{1}{2}\left(\frac{\sqrt{1-\frac{v^{2}}{c_{1.1}^{2}}}}{\sqrt{1-\frac{v_{p}^{2}}{c_{1.1}{ }^{2}}}}+\frac{\sqrt{1-\frac{v^{2}}{c_{1.2^{2}}}}}{\sqrt{1-\frac{v_{p}^{2}}{c_{1.2}{ }^{2}}}}\right)=1.0002446
$$

Change of $\mathbf{G}$ due to aphelion:

$$
\Delta G_{a}=\frac{1}{2}\left(\frac{\sqrt{1-\frac{v a^{2}}{c_{1.1}^{2}}}}{\sqrt{1-\frac{v^{2}}{c_{1.1}^{2}}}}+\frac{\sqrt{1-\frac{v a^{2}}{c_{1.2}^{2}}}}{\sqrt{1-\frac{v^{2}}{c_{1.2}^{2}}}}\right)=1.0002354
$$

Giving the total:

$$
\begin{aligned}
& \Delta G=\frac{1}{2}\left(\Delta G_{p}+\Delta G_{a}\right)=1.00024 \\
& \mathrm{v}=\text { orbital velocity of Earth at semi-major axis }=\mathbf{2 9 7 8 4 . 4 8 5 ~} \mathrm{m} / \mathrm{s} \\
& v_{p}=\text { orbital velocity of Earth at perihelion }=30037.537 \mathrm{~m} / \mathrm{s}
\end{aligned}
$$

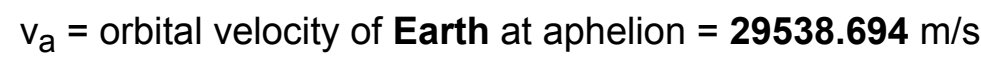

$C_{1.2}=$ space orbital velocity of the $1 / 2 R$ Sun discontinuity $=151.266563 * 10^{3} \mathrm{~m} / \mathrm{s}$

$\mathrm{C}_{1.1}=$ space orbital velocity of the $2 / 3 \mathrm{R}$ Sun discontinuity $=230.556106 * 10^{3} \mathrm{~m} / \mathrm{s}$

Velocities $c_{1.1}$ and $c_{1.2}$ have been calculated in the Quantization of the Sun chapter.

For a mean $\mathbf{G}$ of $6.673899 * 10^{-11} \mathrm{~m}^{3} / \mathrm{kgs}^{2}$ and $\Delta \mathrm{G}=1.00024$, the amplitude of oscillation is 1.60173576 * $10^{-14} \mathrm{~m}^{3} / \mathrm{kgs}^{2}$.

Measurements of $\mathbf{G}$ on Earth indeed show sinusoidal oscillation, although in previous analysis it has been correlated with the $5.9 y\left(5.899 \pm 0.062\right.$ y) period oscillation component of Earth's length of day (LOD) ${ }^{19}$.

Howerver, calculated amplitude of yearly oscillation $\left(1.60173576 * 10^{-14} \mathrm{~m}^{3} / \mathrm{kgs}^{2}\right)$ agrees very well with the amplitude obtained from measurements $\left(1.619 \pm 0.103 * 10^{-14} \mathrm{~m}^{3} / \mathrm{kgs}^{2}\right)$. 


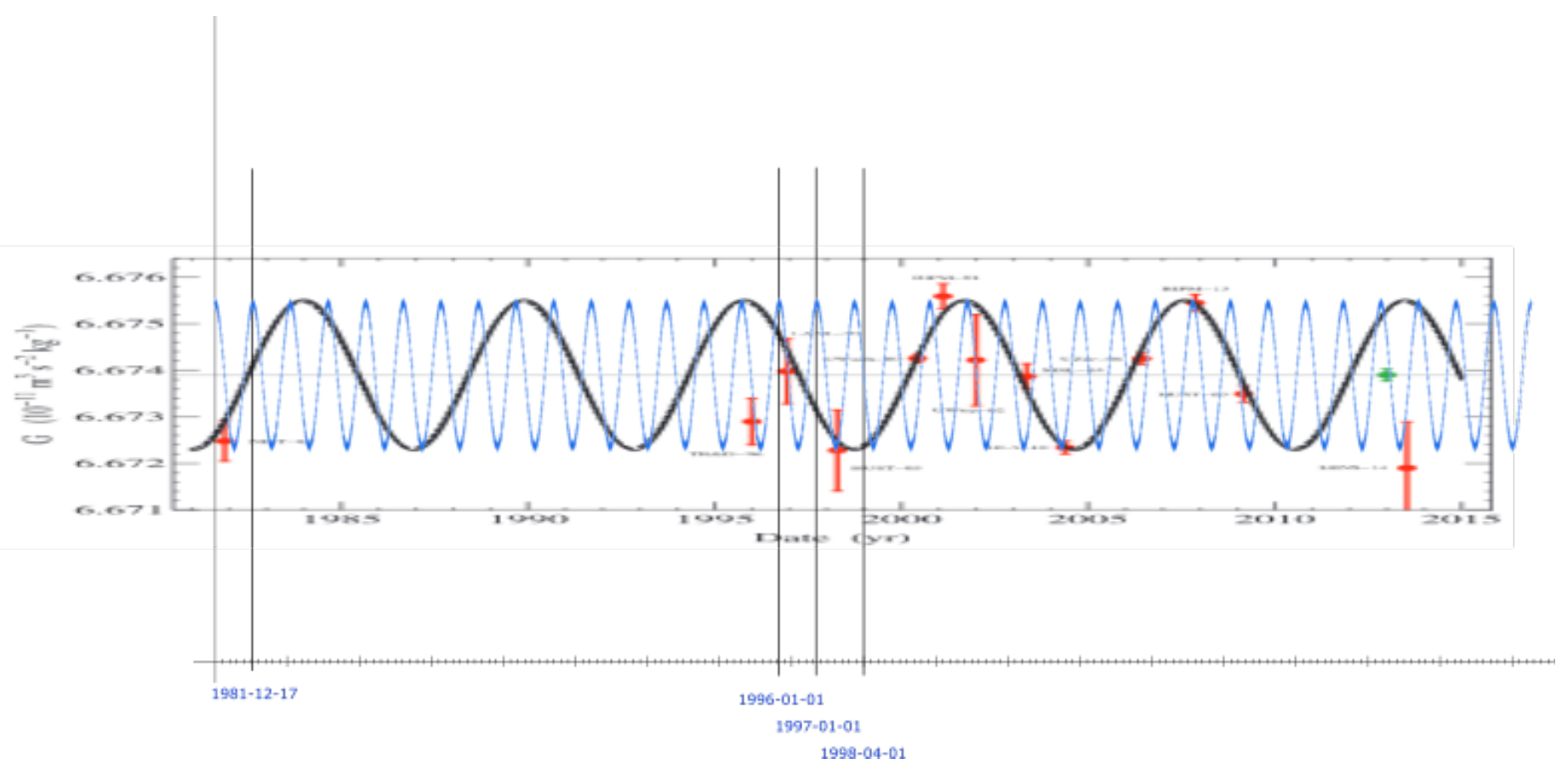

Fig. 1: Oscillation of the gravitational constant

Fig 1. shows yearly oscillation (blue) superimposed on the $5.9 \mathrm{y}$ oscillation from previous analysis (black). Red crosses are previously measured values of $\mathbf{G}$, plotted with uncertainties.

Yearly oscillation is obviously a better fit, but when linked to orbits of the Earth around the Sun (orbital data taken from NASA Horizons On-Line Ephemeris System ${ }^{20}$ ) a phase shift of $\approx 0.6167 y$ (golden ratio?) is required to match Fig 1. (without the shift the correlation is less convincing with all measurements taken into account).

Interestingly, with the influence of the Sun removed, leaving only planetary gravitational interactions, best fit requires no phase shift: 


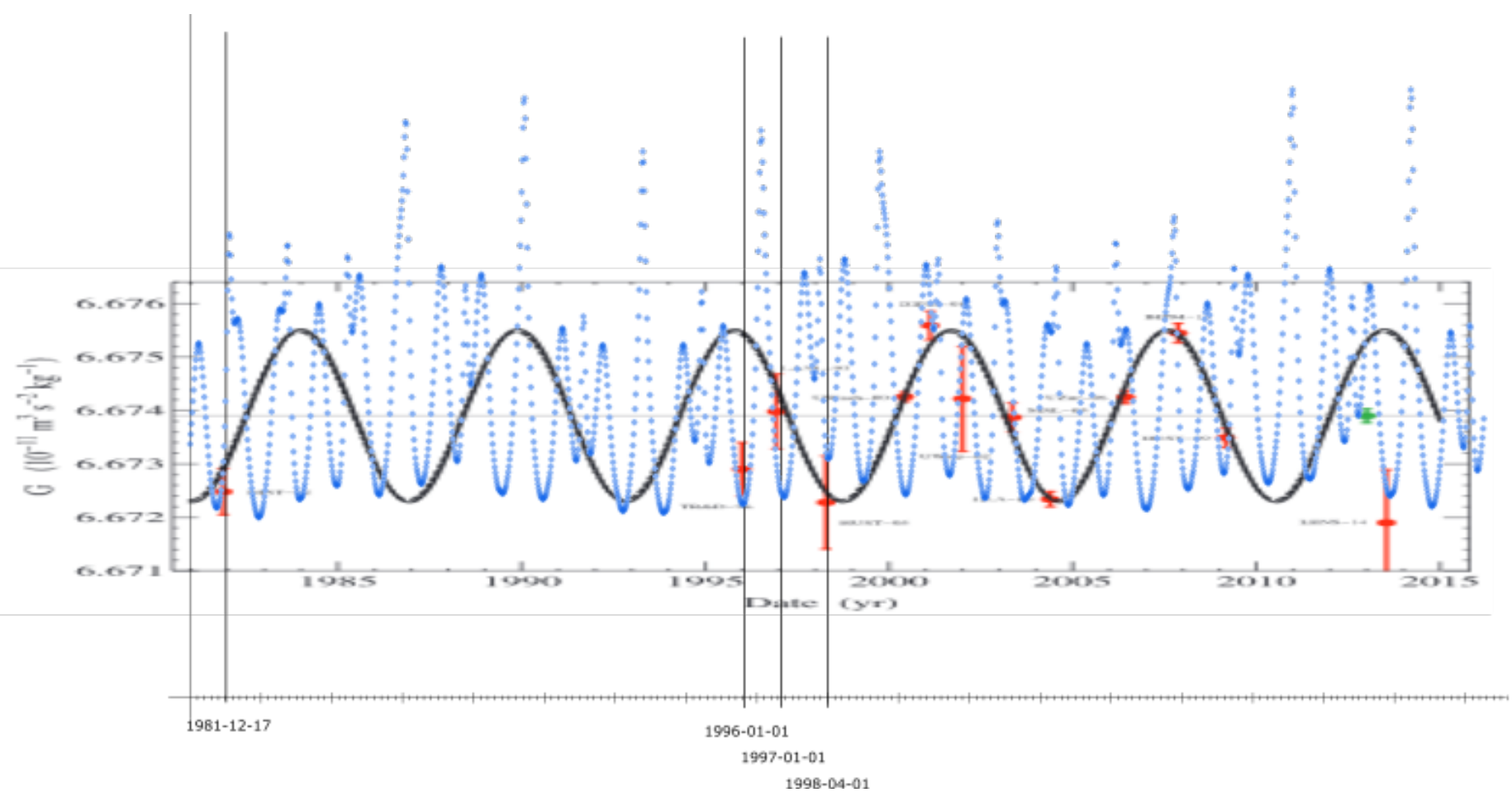

Fig. 2: Oscillation of the gravitational constant

The 5.9y period oscillation in LOD is equal to a solar orbit in 2:1 resonance with Jupiter and a 5:1 resonance with Saturn. If Mars (which is in 1e configuration) is entangled with 1e of Jupiter, the Earth (2e configuration) should be entangled with the other $1 \mathrm{e}$ of Jupiter and 1e of Saturn.

The resonant orbital (outer edge of the main asteroid belt) must be the event horizon (which should currently be in a collapsed form - similar to larger horizons collapsed into dwarf planets) of such entanglements.

This is (or rather, a memory of - due to neutralization of EM force) a magnetic spin entanglement between particles (notice the anti-alignment of magnetic fields between Earth and Jupiter/Saturn), and thus should have a signature in geomagnetic field. 


\section{Quantization of surface radii}

Here are, somewhat empirically determined, equations for quantization of surface radii in the Solar System.

Neutral equatorial radius for outer planets:

$$
R=\frac{K_{2}}{r^{2}} M \frac{1}{2^{(2-p)}}\left[\left(\frac{1}{10^{1}}\right)^{(4-N)} 3^{(3-p)} \frac{1}{n^{(p-1)}}\right]^{(s-1)}
$$

Neutral equatorial radius for inner planets:

$$
R=\frac{r^{2}}{K_{1}} \frac{1}{M} n^{(1-p)} 2^{(N-1)}\left[2^{(4-n)} \frac{1}{3^{(1-p)}}\right]^{(s-1)}
$$

Since both $\mathbf{r}$ and $\mathbf{M}$ (gravity) are quantized, it follows that $\mathbf{R}$ is quantized too by the $\mathbf{K}$ constant - other factors $(\mathbf{n}, \mathbf{p}, \mathbf{s}, \mathbf{N})$ are integers.

The above may be understood as the invariant component of the radius during the cycle. Current radius includes a small correction due to oscillation in electric polarization (charge), value of which evolves weakly during the cycle state.

Current equatorial radius for outer planets:

$$
\begin{gathered}
R=\frac{K_{2}}{r^{2}} M \frac{1}{2^{(2-p)}} \frac{1}{10^{1}}\left(\frac{3^{2}}{10^{1}}\right)^{\left(2-K_{\varphi}\right)} K_{\varphi}\left[\frac{1}{n^{(p-1)}}\right]^{(s-1)} \\
K_{\varphi}=10^{-\left[\sin \left(180^{\circ}-\Delta_{\varphi}\right)^{(p \bmod 2)} \cos \left(180^{\circ}-\Delta_{\varphi}\right)^{(1-p \bmod 2)}\right]} \\
\Delta_{\varphi}=\varphi_{0}-\varphi_{1}
\end{gathered}
$$

Current equatorial radius for inner planets:

$$
\begin{gathered}
R=\frac{r^{2}}{K_{1}} \frac{1}{M} n^{(1-p)}\left(2+K_{\varphi}\right)\left[2^{(2-p)} 10^{0}\left(\frac{10^{0}}{3^{2}}\right)^{-K_{\varphi}}\right]^{(s-1)} \\
K_{\varphi}=10^{0} \cos \left(180^{\circ}-\Delta_{\varphi}\right)^{(p \bmod 2)} \sin \left(180^{\circ}-\Delta_{\varphi}\right)^{(1-p \bmod 2)}
\end{gathered}
$$




$$
\Delta_{\varphi}=\varphi_{0}-\varphi_{1}
$$

$$
\begin{array}{r}
\mathrm{K}_{2}=4885811.341 \mathrm{~m}^{3} / \mathrm{kg} \\
\mathrm{K}_{1}=2.385039177 * 10^{-9} \mathrm{~m} / \mathrm{kg} \\
\mathrm{M}=\text { total mass } \\
\mathrm{r}=\text { orbital radius } \\
\mathrm{N}=\text { shell number } \\
\mathrm{s}=\text { number of particles in a sub-shell } \\
\mathrm{p}=\text { state of quantization } \\
\mathrm{n}=\text { shell energy level }
\end{array}
$$

\begin{tabular}{|c|c|c|c|c|c|c|c|c|c|c|}
\hline $\mathbf{N}$ & $\mathbf{n}$ & planet & $M(k g)$ & $r(m)$ & $\mathbf{s}$ & p & $\Delta_{\varphi}\left({ }^{\circ}\right)$ & neutral $\mathbf{R}(\mathbf{k m})$ & current $\mathbf{R}(\mathbf{k m})$ & $\mathbf{R}(\mathbf{k m})$ \\
\hline 2 & 5 & Neptune & $1.02413 * 10^{\wedge} 26$ & 4495060000000 & 1 & 2 & 36.7084 & 24764 & 24764 & 24764 \\
\hline 2 & 5 & Uranus & $8.6813 * 10^{\wedge} 25$ & 2872460000000 & 1 & 1 & 233.1506 & 25703 & 25559 & 25559 \\
\hline 2 & 3 & Saturn & $5.6834 * 10^{\wedge} 26$ & 1433530000000 & 2 & 1 & 0.2 & 60806 & 60268 & 60268 \\
\hline 1 & 1 & Jupiter & $1.89819 * 10^{\wedge} 27$ & 778570000000 & 2 & 1 & 109.422 & 68848 & 71492 & 71492 \\
\hline 2 & 5 & Mercury & $3.3011 * 10^{\wedge} 23$ & 57910000000 & 2 & 2 & 172.3047 & 2555.7 & 2439.7 & 2439.7 \\
\hline 2 & 3 & Venus & $4.8675 * 10^{\wedge} 24$ & 108210000000 & 1 & 0 & 0 & 6051.8 & 6051.8 & 6051.8 \\
\hline 2 & 3 & Earth & $5.9723 * 10^{\wedge} 24$ & 149600000000 & 2 & 1 & 90.3135 & 6284.72 & 6378.14 & 6378.14 \\
\hline 1 & 10 & Mars & $6.4171 * 10^{\wedge} 23$ & 227920000000 & 1 & 2 & -91.9957 & 3394.1 & 3396.2 & 3396.2 \\
\hline
\end{tabular}

$\Delta_{\varphi}=$ angle between spin momenta of a particle pair occupying the shell (in case of a single particle - induced pair by splitting of a maximum)

For the state $6 \mathrm{p} 4 \mathrm{n}$ :

Note the quantization of $\Delta_{\varphi}$. For inner planets, it is quantized by $90^{\circ}$ (any deviation may be due to higher order oscillation).

For outer planets, the quantum is reduced to $1 / 5$ of this value, $18^{\circ}$, suggesting, perhaps that the equation for outer planets should be modified, or, instability in entanglement.

Thus, to obtain $90^{\circ}$ quantization, one only needs to multiply $\Delta_{\varphi}$ (quantized by $18^{\circ}$ ) with 5 , revealing how it is entangled (assuming anti-alignment) with one of inner planets:

\begin{tabular}{lcc} 
planet & standardized $\Delta_{\varphi}\left({ }^{\circ}\right)$ & entanglement \\
\hline Neptune & $(5 * 36) \% 360=180$ & Venus \\
\hdashline Uranus & $(5 * 234) \% 360=90$ & Mars
\end{tabular}


Here, Neptune/Venus, Uranus/Mars and Saturn/Mercury entanglement should not be surprising due to matching configurations $-1 \mathrm{e} / 1 \mathrm{e}, 1 \mathrm{e} / 1 \mathrm{e}$ and $2 \mathrm{e} / 2 \mathrm{e}$.

The entanglement of Jupiter with Venus instead of Earth might be the consequence of ${ }^{10} \mathrm{C}$ instability, or a phase shift in entanglement.

\subsection{Radius of the Sun and its correlation with proton radius}

Original composition of the Sun is $\mathbf{6}$ protons $+\mathbf{4}$ neutrons. However, $\mathbf{6}$ positrons worth of charge (inner planets) have been removed to balance the electrons (outer planets).

This makes the Sun neutral:

$$
6 *\left(-\frac{1}{3} e+2 * \frac{2}{3} e\right)+4 *\left(2 *-\frac{1}{3} e+\frac{2}{3} e\right)-6 e=0
$$

The Sun still consists of both positive and negative charges but their spin effects on radius cancel out. The radius is thus:

$$
\begin{gathered}
R=R_{2}+R_{1} \\
R_{2}=\frac{K_{2}}{r_{2}^{2}} M_{2} \frac{1}{2^{\left(2-p_{2}\right)}}\left[\left(\frac{1}{10^{1}}\right)^{\left(4-N_{2}\right)} 3^{\left(3-p_{2}\right)} \frac{1}{n_{2}\left(p_{2}-1\right)}\right]^{\left(s_{2}-1\right)} \\
R_{1}=\frac{r_{1}^{2}}{K_{1}} \frac{1}{M_{1}} n_{1}^{\left(1-p_{1}\right)} 2^{\left(N_{1}-1\right)}\left[2^{\left(4-n_{1}\right)} \frac{1}{3^{\left(1-p_{1}\right)}}\right]^{\left(s_{1}-1\right)}
\end{gathered}
$$

where $\mathbf{R}_{\mathbf{2}}$ is the sum radius of negative quarks and $\mathbf{R}_{\mathbf{1}}$ is the sum radius of positive quarks. 

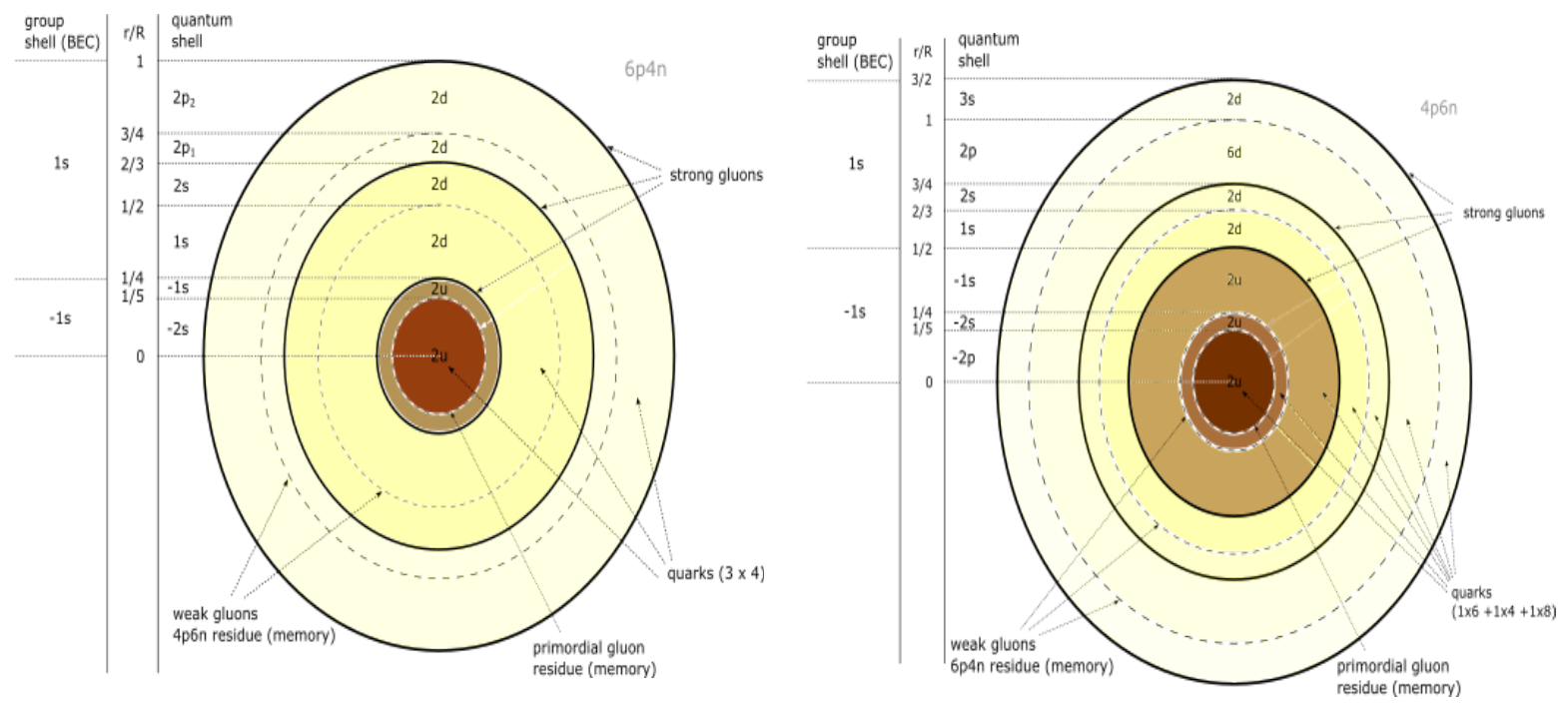

Fig. 2: Sun partitioning in: a) $6 p 4 n$ state b) $4 p 6 n$ state ( $R=$ radius in $6 p 4 n$ state)

As shown on Fig. 2, without $\mathbf{6}+\mathrm{e}$ charges, the Sun is a sum neutron consisting of $\mathbf{6}$ layers, $\mathbf{4}$ layers containing pairs of negative [down equivalent] quarks and 2 layers (inner and outer core) containing pairs of positive [up equivalent] quarks. Due to condensation, this is the equivalent of a single neutron so 8 negative quarks can be grouped into a single sub-shell as 2 negative quarks, while 4 positive quarks can be grouped into another sub-shell as a single positive quark $(8 / 4=2 / 1)$.

Thus, the parameter $s_{2}=2$, while $s_{1}=1$.

The energy of these two sub-shells must be equal, so $\mathbf{M}_{\mathbf{2}}=\mathbf{M}_{\mathbf{1}}=\mathbf{M}$.

For equal impact on radii, this must be satisfied:

$$
\frac{K_{2}}{r_{2}{ }^{2}} M_{2}=\frac{r_{1}^{2}}{K_{1}} \frac{1}{M_{1}}
$$

Since $\mathbf{M}_{\mathbf{2}}=\mathbf{M}_{\mathbf{1}}=\mathrm{M}$ :

$$
\begin{gathered}
\frac{K_{2}}{r_{2}{ }^{2}} M=\frac{r_{1}^{2}}{K_{1}} \frac{1}{M}=\sqrt{\frac{K_{2}}{K_{1}}} \frac{r_{1}}{r_{2}} \\
R=\sqrt{\frac{K_{2}}{K_{1}}} \frac{r_{1}}{r_{2}}\left[\frac{1}{2^{\left(2-p_{2}\right)}}\left(\frac{1}{10^{1}}\right)^{\left(4-N_{2}\right)} 3^{\left(3-p_{2}\right)} \frac{1}{n_{2}\left(p_{2}-1\right)}+n_{1}^{\left(1-p_{1}\right)} 2^{\left(N_{1}-1\right)}\right]
\end{gathered}
$$

Here $\mathbf{p}_{\mathbf{1}}$ corresponds to number of major (strong) gluons, $\mathbf{p}_{\mathbf{2}}$ to weak gluons, $\mathbf{N}$ continues increasing from Mercury (2) so $N_{1}=3$ and $N_{2}=4$ :

$$
p_{2}=2, N_{2}=4, n_{2}=3^{2}=9
$$




$$
p_{1}=3, N_{1}=3, n_{1}=3
$$

Sun radius then becomes:

$$
R=\sqrt{\frac{K_{2}}{K_{1}}} \frac{r_{1}}{r_{2}}\left[\frac{1}{3}+\left(\frac{2}{3}\right)^{2}\right]
$$

Here, ratio $\mathbf{r}_{\mathbf{1}} / \mathbf{r}_{\mathbf{2}}$ is equal to the ratio of orbital radii of outermost electron (Neptune) and outermost positron (Mars).

This gives $R=694271.2405 \mathrm{~km}$.

Radius of the sum $\mathbf{U}_{\mathbf{1}}$ scale proton can be obtained by raising the quark factors of $\mathbf{R}$ to the power of $\mathbf{2}$. This is due to the fact that the removal of a negative down quark reduces the negative radius $9\left(3^{2}\right)$ times, while the addition of a positive up quark reduces the positive radius $\mathbf{3} / \mathbf{2}$ times. Distance between charges increases (due to greater difference between them) so total radius is decreased by the sum of these factors.

$$
R_{p_{1}}=\sqrt{\frac{K_{2}}{K_{1}}} \frac{r_{1}}{r_{2}}\left[\frac{1}{3}+\left(\frac{2}{3}\right)^{2}\right]\left[\left(\frac{1}{3}\right)^{2}+\frac{2}{3}\right]=\sqrt{\frac{K_{2}}{K_{1}}} \frac{r_{1}}{r_{2}}\left[\frac{1}{3}+\left(\frac{2}{3}\right)^{2}\right]^{2}
$$

Radius of a standard proton $\left(\mathbf{U}_{0}\right.$ scale $)$ can now be obtained through this equation:

$$
\frac{R_{p_{1}}}{r_{1}}=\frac{N * R_{p}}{r_{U_{0}}}
$$

Where $\mathbf{r}_{\mathbf{1}}$ is the Solar System charge radius (Neptune's orbit), $\mathbf{N}$ is the number of nucleons in the Solar System, $\mathbf{R}_{\mathrm{p}}$ is the standard proton radius and $\mathbf{r}_{\mathbf{0}}$ is the standard Carbon-10 (Carbon-12) charge radius.

Using Sun radius $\mathbf{R}$ obtained above, this gives:

$$
R_{p}=R_{p_{0}}=\frac{1}{10} \frac{R}{r_{1}}\left[\left(\frac{1}{3}\right)^{2}+\frac{2}{3}\right] r_{U_{0}}=0.840905616 * 10^{-15} \mathrm{~m}
$$

\section{2. $\Delta_{\varphi}$ validation}

Dominant magnetic field in outer planets may be generated by positive charge, while in inner planets by negative.

In any case, $\Delta_{\varphi}$ may also correspond to angle between magnetic dipoles. 


\subsubsection{Mercury}

$\Delta_{\varphi}$ obtained for Mercury corresponds to $\downarrow \uparrow$ spin configuration. This is generally consistent with a low strength magnetic field. However, current low strength of Mercury's magnetic field should not be attributed to such configuration as the primal source is subdued.

\subsubsection{Venus}

$\Delta_{\varphi}$ for Venus suggests extremely strong primal magnetic field.

\subsubsection{Earth}

Earth's magnetic dipole is not axial, revealing a primal quadrupole configuration, as expected with $2 \mathrm{e}$ configuration. Considering the movement of north and south dip poles and attributing it to imminent collapse, in the primal configuration two major (inner and outer) dipoles may very well be separated by $90^{\circ}$, equal to calculated $\Delta_{\varphi}$

This configuration may have been fossilized in the inner core anisotropy, as shown on Fig. 2.

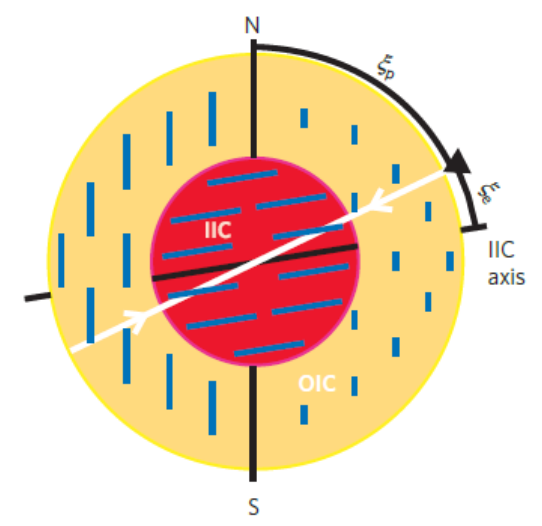

Fig. 2: Equatorial anisotropy of the Earth's inner core 21

\subsubsection{Mars}

Obtained $\Delta_{\boldsymbol{\varphi}}$ shows primal dipole configuration of Mars is mirroring the Earth's. The configuration may be verified once the magnetic field is restored.

\subsubsection{Jupiter}

$\Delta_{\varphi}\left(109^{\circ}\right)$ corresponds to $\uparrow \rightarrow$ configuration, and is consistent with observation: 


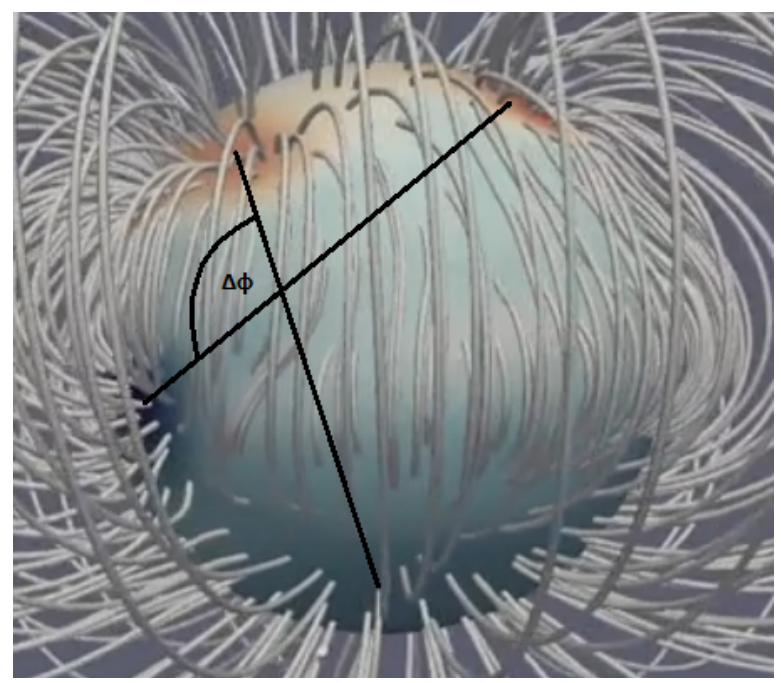

Fig. 3: Magnetic field of Jupiter 22

\subsubsection{Saturn}

Saturn's dipole field is aligned with the rotation axis and highly axisymmetric, while quadrupole and higher components are significantly weaker.

This is consistent with $\uparrow \uparrow$ configuration suggested by $\Delta_{\varphi}\left(0^{\circ}\right)$.

\subsubsection{Uranus}

Dipole center is significantly offset from the center of the planet. Assuming primal core-dipole entanglement, $\boldsymbol{\Delta}_{\boldsymbol{\varphi}}$ may be interpreted as the angle between the equator and dipole rotated by such angle that the [shortest] distance from dipole center to equator $(\mathbf{x})$ is equal to distance from planet surface to the intersection of the rotated axis and axis translated to center, as shown on Fig. 4. 


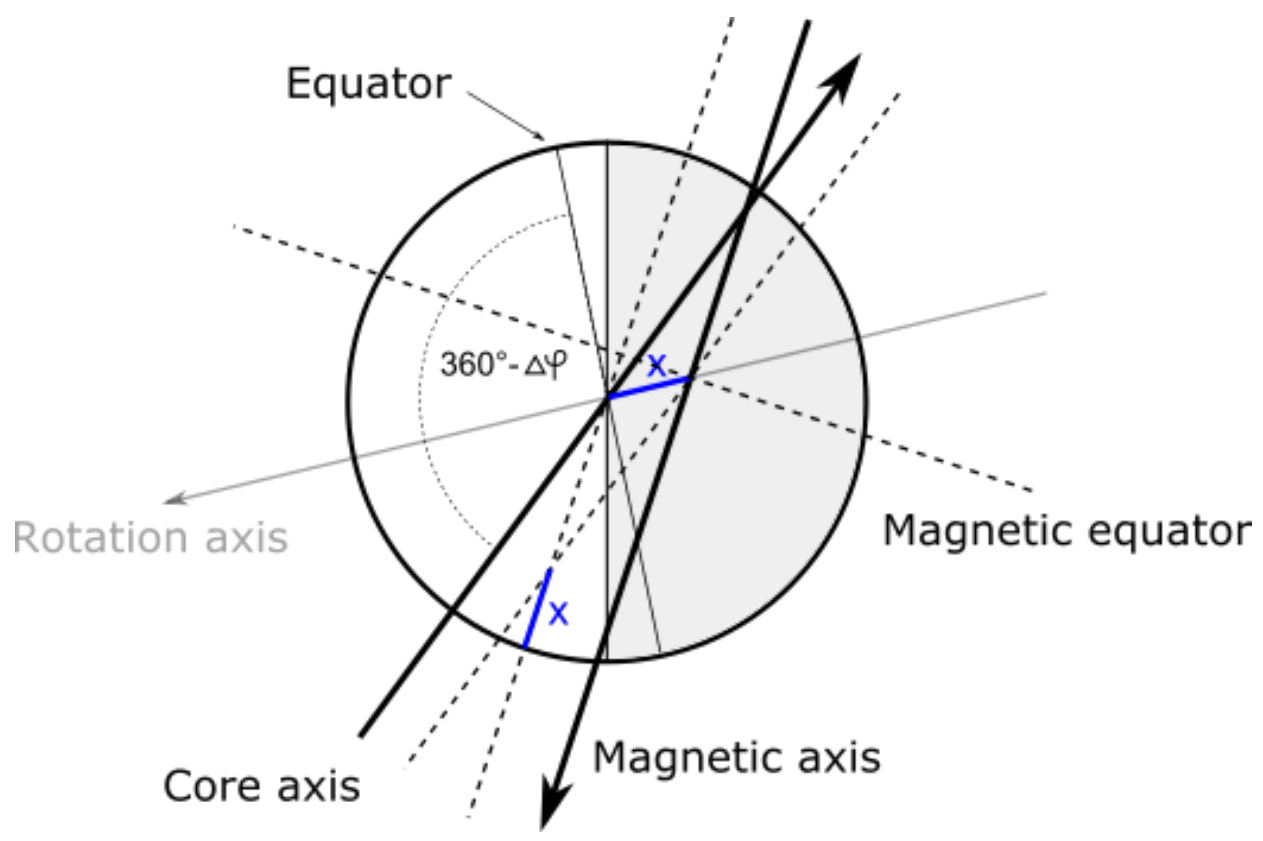

Fig. 4: Uranus' magnetic field model

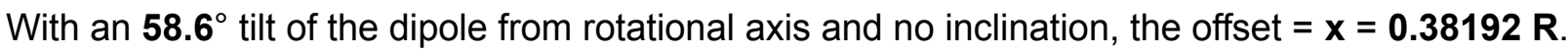

With an inclination of the dipole from rotational axis equal to $1.82^{\circ}$, the offset is equal to $0.353 \mathbf{R}$, in agreement with NASA/GSFC-O 3 model.

\subsubsection{Neptune}

Similar to Uranus, the dipole is significantly offset from the center. Using the same method as in case of Uranus, one obtains the dipole shown on Fig. 5. 


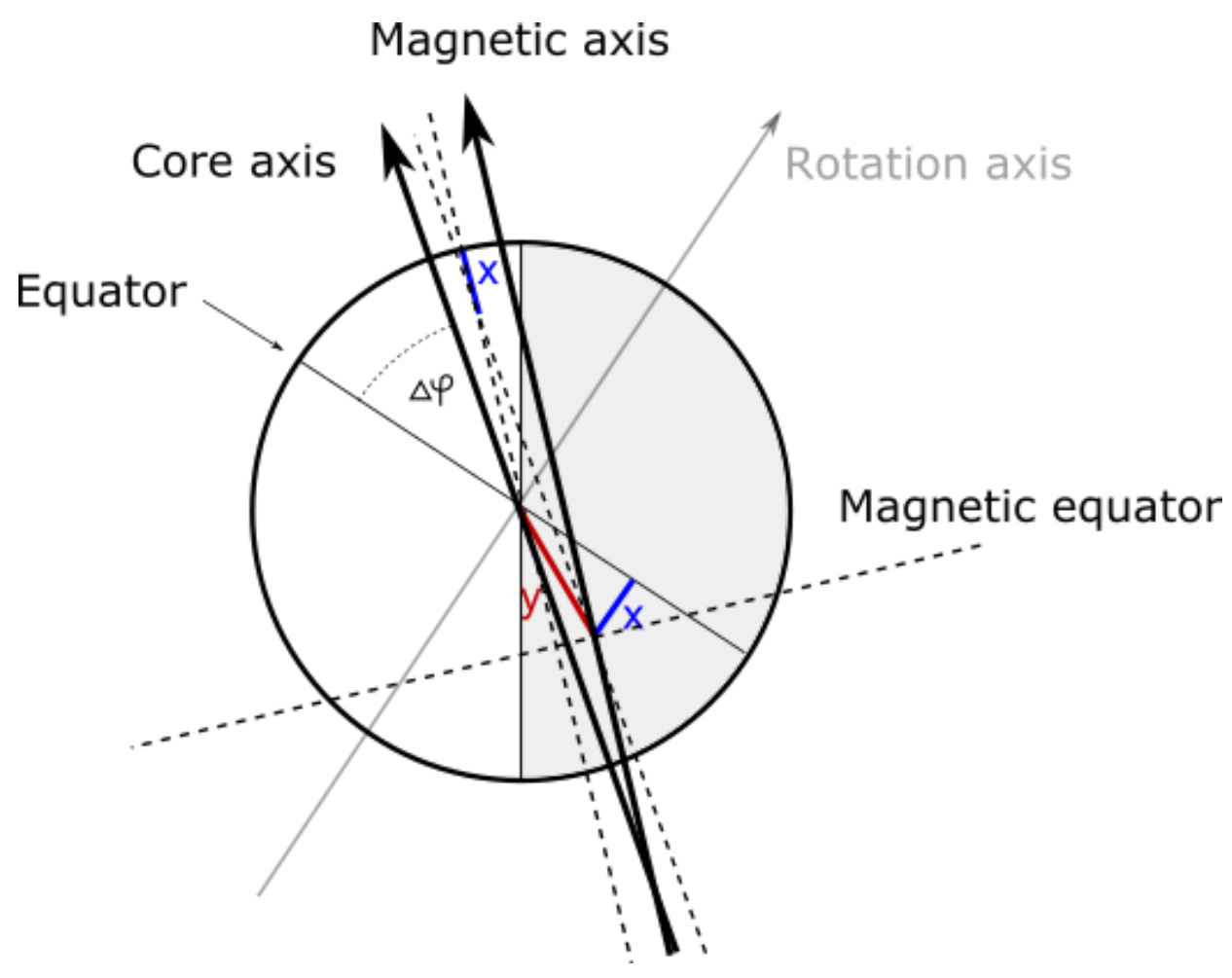

Fig. 5: Neptune's magnetic field model

With an $46.9^{\circ}$ tilt of the dipole from rotational axis, with no inclination, the offset is equal to $0.12193 \mathbf{R}$.

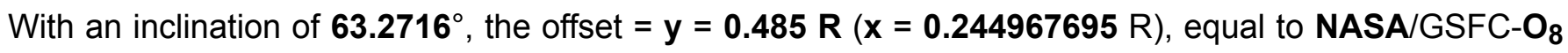
model. 


\section{Earth, as a particle}

Orbits of discontinuities (gravitational maximums) and their capacities are quantized and this horizontal entanglement between planets is evident through the $\mathbf{h}$ constant.

It would be intuitive to expect that gravity within the planet follows the same pattern. Here, however, due to vertical (spin momentum) entanglement with a planet of a distant system one can expect a larger oscillation of the spin $\mathbf{h}$ constant between the planets.

For positive bodies (terrestrial planets), gravity should generally increase with depth, down to the inner gravitational maximum, radius of the inner core.

For Earth, surface gravity of this maximum is equal to the surface maximum of the Sun $-274 \mathrm{~m} / \mathrm{s}^{2}$.

Down to the inner core, for the naked maximum:

$$
\begin{gathered}
g v r=n h \\
g r^{2}=n T \frac{h}{2 \pi} \\
g r^{2}=n T \hbar, \\
g=n T \frac{\hbar}{r^{2}},
\end{gathered}
$$

where $\mathbf{T}$ is the rotation period at radius $\mathbf{r}$. In equilibrium, $\mathbf{T}$ at surface for a solid body is:

$$
T=T_{0}
$$

while real radius of the planet is:

$$
R=R_{0}
$$

If $\mathbf{n T}$ is const.:

$$
g=\frac{\hbar_{m g}}{m} \frac{R^{2}}{r^{2}}
$$

For Earth:

$$
T=24 h=86400 s
$$




$$
R_{0}=\text { const. }=6307105 \mathrm{~m}
$$

Down to the inner core radius $\mathbf{r}_{\mathbf{c}}$ :

$$
\begin{array}{r}
n=n_{s}=1 \\
g=86400 \frac{\hbar}{r^{2}}=\frac{G m_{\text {img }}}{r^{2}} \approx \frac{G M}{r^{2}} \\
\mathrm{G}=\text { gravitational constant }=6.674 * 10^{-11} \mathrm{~m}^{3} / \mathrm{kgs}^{2} \\
\mathrm{M}=\text { total gravitational mass of Earth }=5.9723 * 10^{24} \mathrm{~kg} \\
\hbar=\hbar_{1}=4.613325255 * 10^{9} \mathrm{~m}^{3} / \mathrm{s}^{3}
\end{array}
$$

\begin{tabular}{|l|l|l|l|}
\hline $\mathbf{n}$ & layer & radius $(\mathrm{m})$ & gravity $\left(\mathrm{m} / \mathrm{s}^{2}\right)$ \\
\hline 1 & img surface & 6371000 & 9.82 \\
\hline 1 & img surface perihelion & 6357000 & 9.86 \\
\hline 1 & real surface & 6307105 & 10.02 \\
\hline 1 & outer core & 3282185 & 37 \\
\hline 1 & transition zone (induced charge) & 1705704 & 137 \\
\hline 1 & inner core $=r_{C}$ & 1206115 & 274 \\
\hline 1 & transition zone & 852852 & 137 \\
\hline $2 ?$ & inner inner core & 603058 & $274 ?$ \\
\hline & & & 2 \\
\hline
\end{tabular}

Below $\mathbf{r}_{\mathbf{c}}(\mathbf{1 2 0 6 1 1 5} \mathrm{m})$ gravity becomes:

$$
g=n^{2} \frac{1}{T} \frac{1}{\hbar_{2}} r^{2}
$$

At $\mathbf{r}_{\mathbf{c}}$ (event horizon):

$$
n T \frac{\hbar_{1}}{r^{2}}=n^{2} \frac{1}{T} \frac{1}{\hbar_{2}} r^{2}, \rightarrow \hbar_{2}=6.144878706 * 10^{4} \mathrm{~ms}
$$

At the event horizon gravity is independent of period and radius:

$$
g=\sqrt{\frac{\hbar_{1}}{\hbar_{2}}}
$$

Below $\mathbf{r}_{\mathbf{c}}$ the space-time gradient inverts and gravity is decreasing until it reaches minimum, afterwards increasing again to next maximum, continuing the oscillation. 


\section{Earth, as a living organ(ism)}

As it will be shown, Earth is not only behaving as a living organism, it is a living organism in the same sense as any life-form on its surface is a living organism.

As hard as it may be for a creature of extroverted nature to accept it, Solar System is also an individual organism, and, relative to that system, Sun and planets are the organs.

Obeying the principle of self-similarity, each living organ has an active core, replicating the role of the Sun in the Solar System to localized space-time.

\subsection{Introduction to internal structure}

Blood arteries are underground tubes, blood being the flowing magma and water, carrying nutrients.

$$
\text { Proper interpretation of lava solidification is coagulation of blood. }
$$

Veins are tubes filled with oil.

Complex life and network of interconnected diversity is not limited to surface, which should be understood not only as epidermis but a breeding ground for cultivation of precursor neuron cells and proteins of a planet.

Even though the most expressed organ of this organism is a layered brain, it has to have other organ equivalents necessary for the function of that brain.

The core of a planet has a role of the heart, providing energy necessary to pump the blood all around the planet.

Geyser eruptions provide one way to probe the heart rate.

\subsection{Age and 3rd order period}

The lifetime of Earth is quantized and can be calculated through its frequency of existence.

$$
\Delta T_{E}=n \frac{1}{f_{x}}=n T_{x}
$$


For $n=\mathbf{2 8 4 0}$, and determined $T_{x}$ of the $3 r d$ order general oscillation equal to $1.512 * 10^{6}$ years:

$$
\Delta T_{E}=n T_{x}=4.29408 * 10^{9} \text { years }
$$

There are at least 3 ways to calculate the $3 \mathrm{rd}$ order period of existence cycle $\mathbf{T}_{\mathrm{x}}$, all giving the same result:

\subsubsection{Decay rate of $10 \mathrm{C}$ at $\mathrm{U}_{1}$ scale}

Current Solar System state is ${ }^{10} \mathrm{C}$. Half-lives of elements are inverted relative to the shared decay product between adjacent vertical scales.

Thus, this element has a half-life equal to ${ }^{10} \mathrm{Be}$ at $\mathrm{U}_{0}$ scale.

Several measurements of ${ }^{10} \mathrm{Be}$ half-life have been performed.

In exacmple, in 1987. it was measured to be $1.51 \pm 0.06 * 10^{6}$ years $^{23}$.

In 2009. it was measured to be $1.388 \pm 0.018 * 10^{6}$ years $^{24}$.

Even though the half-life of $\mathbf{U}^{\mathbf{0}}$ elements should be consistent during the existence cycle of $\mathbf{U}^{\mathbf{1}}$, it changes during the transition between cycles - $\mathbf{T}_{\mathrm{X}}$ should be understood as the mean value.

For that reason, I do not consider the value from 2009. as the average value through the lifetime of the Solar System. It will be shown later that this value is $1.512 * 10^{6}$ years.

This is the 3rd order period of the existence cycle of the Solar System, and consequently, Earth.

\subsubsection{Heart rate}

The average heart rate of Earth can be calculated from the global average period between geyser eruptions:

$$
\left\langle T_{g}\right\rangle_{T}=6.6 \text { hours }
$$

Note that Earth is in a superposition of quantum states and our [energy] scale is too low to disturb that superposition.

The fact that we can measure these rates [and anything else in the Solar System], with high precision and not disturbing the the system, shows that, while uncertainties in measurement are fundamental, the size 
i of uncertainty is a measurement problem arising from inadequate scale of observational energy, a relative quantity (Planck's constant, $\hbar$, as a dimensional constant between entangled properties, must be a relative, not absolute constant).

Note that this also shows the nature of superposition - as postulated by $\mathbf{C R}$, a system cannot be in multiple states at the same time unless these are separated in space, and cannot be in multiple states at the same space unless they are separated in time.

For Earth heart rate $=$ my rest heart rate $=76 \mathrm{bpm}$ :

1 Earth scale minute $=76 * 6.6=495$ hours $=20.625$ days

Given the number of heartbeats $\mathrm{EH}_{3 / 3}\left(1 * 10^{9}, 4 * 10^{9}\right)=2 * 10^{9}$ and scale invariance of heartbeats, the period is:

$$
T_{x}=2 * 10^{9} * 6.6=1.32 * 10^{10} h=1.51 * 10^{6} \text { years }
$$

This number of heartbeats with a heart rate of $\mathbf{7 6} \mathrm{bpm}$ corresponds to a human lifespan of $\mathbf{5 0}$ years. This, I consider as the global average human lifespan over the course of evolution on Earth's surface.

With such number of heart beats ${ }^{25}$ (between incarnations), the Earth would belong to, not only mammalian species, but homo species.

The 3rd order cycle of the Solar System $\left(1.512 * 10^{6}\right.$ years) can thus be interpreted as evidence of evolutionary entanglement - a man is in its path of evolution between the standard scale carbon atom and the Solar System (large scale carbon atom).

However, even though I believe the soul of such species as Earth can reuse a discarded body, I find it unlikely that Earth's soul is replaced every $\mathbf{1 . 5 1 2}$ million years.

Replacement likely occurs with the end of the 1st order cycle, while higher order cycles should be interpreted as temporary loss of consciousness.

Such collapses are normal during embryonic development, but they should occur even in adults (of all species) albeit with such magnitude that they are generally unnoticeable.

To species accustomed to the concept of birth and extroverted nature it might appear that Earth never fully develops.

This is most certainly not the case - life past the embryonic form to us always results in the change of 
environment, but this is only due to inadequacy of current environment to ensure the continuity of progressive evolution, one which includes growth of the physical form.

Once extroverted intelligence evolves into, more energy efficient, introverted intelligence with low energy requirements, there is no need for physical growth or reason for most of conventional physical organs.

Spherical form is, thus, a pinnacle of evolution, rather than an undeveloped form of life, even though it externally manifests itself as a mere particle, or, a piece of rock.

If a man should regard any cosmic phenomena as a deity, it should certainly be Earth, as it would be the one closest to us. A god with whom we are strongly entangled and thus evolutionary depend on.

\subsubsection{Speed of time}

Space-time may be represented by 3 dimensions, one positively polarized (space), one negative (time) and one neutral (event horizon in between).

These 3 dimensions are spatially separated and quantized, but they are entangled and may orbit the same body, such that the orbital velocity of event horizon is:

$$
v_{E H}=\left(v_{S}-v_{T}\right) * C
$$

where $\mathbf{v}_{\mathbf{S}}$ and $\mathbf{v}_{\mathbf{T}}$ are orbital velocities of space and time dimensions, respectively.

3rd order space for Earth is 1-dimensional - the Earth is an inflated quantum of space-time orbiting the Sun. Dimensions of [3rd order] space and time of Earth have been further separated during inflation, but they remain entangled. Space dimension is thus at the Earth's orbital radius.

Time dimension velocity is quantized by $\mathbf{v}_{\mathbf{S}}$ :

$$
\begin{gathered}
v_{T}(n)=\left\{(n+j)+(n-i) \pm[(n+j) *(n-i)]^{-1}\right\}^{-i} *\left\{(n+j) \pm\left[(n+j) *(n-i)^{2}\right]^{-1}\right\}^{-j} * v_{S}(n) \\
n, i, j \in \mathbb{Z} \\
i=n-C_{1}, j=C_{2}-n, i+j=C_{2}-C_{1} \\
C_{1}, C_{2} \in \mathbb{N}
\end{gathered}
$$

The values in square brackets, depending on the sign, give maximum and minimum values of $\mathbf{v}_{\mathbf{T}}$ during the cycle state. The average (mean) $\mathbf{v}_{\mathbf{T}}$ :

$$
v_{T}(n)_{A V G}=[(n+j)+(n-i)]^{-i} *[(n+j)]^{-j} * v_{S}(n)=(2 n+j-i)^{-i} *(n+j)^{-j} * v_{S}(n)
$$




$$
v_{T}(n)_{A V G}=\left(C_{1}+C_{2}\right)^{C_{1}-n} *\left(C_{2}\right)^{n-C_{2}} * v_{S}(n)
$$

For inner planets, in state $6 \mathrm{p} 4 \mathrm{n}$ :

$$
\begin{gathered}
C_{1}=2, C_{2}=3 \\
v_{T}(n)=\left[5+(3 * 2)^{-1}\right]^{-i} *\left[3+(3 * 4)^{-1}\right]^{-j} * v_{S}(n)=\left(5+6^{-1}\right)^{-i} *\left(3+12^{-1}\right)^{-j} * v_{S}(n) \\
i=n-2, j=3-n, i+j=1 \\
v_{T}(n) \approx \frac{1}{v_{n}} * v_{S}(n), v_{n}=v_{n-1}+2^{n-2}, v_{0}=\left(\frac{2}{3}\right)^{-1}=\left(\frac{N}{P}\right)^{-1}
\end{gathered}
$$

Solar System may also be observed as a hydrogen-like atom, where space, time and event horizon dimensions have been split into 4 component vectors (levels).

The event horizon velocity (derived from $\mathbf{v}_{\mathbf{S}}$ and $\mathbf{v}_{\mathbf{T}}$ ), given the orbital energy level vectors for inner $\left(\mathbf{n}_{\mathbf{1}}\right)$, outer $\left(\mathbf{n}_{\mathbf{2}}\right)$ planets and the oscillatory vector $\mathbf{k}$ :

$$
\begin{gathered}
\boldsymbol{n}_{1}=\left[\begin{array}{c}
5 \\
3 \\
3 \\
10
\end{array}\right], \boldsymbol{n}_{2}=\left[\begin{array}{l}
1 \\
3 \\
5 \\
5
\end{array}\right], \boldsymbol{k}=\left[\begin{array}{c}
0 \\
3^{1} \\
3^{2} \\
5^{2}
\end{array}\right] \\
\boldsymbol{v}_{E H}=\left(\boldsymbol{v}_{S}-\boldsymbol{v}_{T}\right) *\left(\frac{\boldsymbol{n}_{1}+\boldsymbol{k} \oplus \boldsymbol{n}_{2}}{10^{1}}+\frac{\boldsymbol{k}}{10^{2}}\right),
\end{gathered}
$$

where $\oplus$ is the sign operator:

$$
\boldsymbol{a} \oplus \boldsymbol{b}=\left[\begin{array}{c}
-1^{a_{1}+1} * b_{1} \\
-1^{a_{2}+1} * b_{2} \\
-1^{a_{3}+1} * b_{3} \\
-1^{a_{4}+1} * b_{4}
\end{array}\right]
$$

Note that the ratio of sums of elements of $\mathbf{n}_{\mathbf{2}}$ and $\mathbf{n}_{\mathbf{1}}$ is:

$$
\frac{\sum n_{2}}{\sum n_{1}}=\frac{14}{21}=\frac{2}{3}=\frac{N}{P}
$$

The event horizon velocity (from $\mathbf{v}_{\mathbf{S}}$ only): 


$$
\begin{gathered}
v_{E H_{0}}(n)=\frac{r_{S}(n)}{r_{M a r s}} v_{S}(n)=\frac{1}{r_{M a r s}} \sqrt{G M * r_{S}(n)} \\
c_{E H}=1 \frac{k m}{s} \\
v_{E H}(n)=v_{E H_{0}}(n)+(-1)^{\left(\delta_{j n, 2}\right)}\left[1+2^{\left(1-\delta_{j, i+1}\right)}-(i j+1) 3^{\left.\left(-2^{\delta_{j, i+1}}\right) \frac{1}{2}\right] c_{E H},}\right.
\end{gathered}
$$

\begin{tabular}{|c|c|c|c|c|c|c|c|c|c|}
\hline $\mathbf{n}$ & Planet & i & $\mathbf{j}$ & $\begin{array}{l}v_{s} \\
(\mathrm{~km} / \mathbf{s})\end{array}$ & $\begin{array}{l}\mathrm{V}_{\mathbf{T}} \mathrm{km} / \mathrm{s} \\
\text { (entanglement) }\end{array}$ & $\sigma_{\mathbf{T}}$ (current value) & $\begin{array}{l}\mathrm{V}_{\mathrm{EH}} \\
\text { (entanglement) } \\
\mathrm{km} / \mathrm{s}\end{array}$ & $\begin{array}{l}\sigma_{E H_{0}} \\
\text { (neutron } \\
\text { correction) }\end{array}$ & $\begin{array}{l}\mathrm{V}_{\text {EH }} \\
\text { (entanglement) } \\
\mathrm{km} / \mathrm{s}\end{array}$ \\
\hline 4 & Mercury & 2 & -1 & 47.36 & 5.47 (Neptune) & $-2^{2 *} 10^{-2}=-0.04$ & $\begin{array}{l}12.033 \\
\text { (Jupiter) }\end{array}$ & +4.73 & 16.77 (Hygiea) \\
\hline 3 & Venus & 1 & 0 & 35.02 & 6.78 (Uranus) & $+2^{1 *} 10^{-2}=+0.02$ & 16.63 (Hygiea) & +1.275 & 17.9 (Ceres) \\
\hline 2 & Earth & 0 & 1 & 29.78 & 9.66 (Saturn) & $+2^{1 *} 10^{-2}=+0.02$ & 19.55 (Vesta) & -1.66 & 17.88 (Pallas) \\
\hline 1 & Mars & -1 & 2 & 24.07 & 13.08 (Jupiter) & $-2^{1 *} \times 10^{-2}=-0.02$ & 24.07 (Mars) & -4.73 & 19.34 (Vesta) \\
\hline
\end{tabular}

where $\delta_{a, b}$ is the Kronecker delta function.

Obviously, the speed of time dimension decreases as the speed of space increases (as predicted by GR) and orbits are quantized and entangled (as predicted by $\mathbf{C R}$ ):

$$
\frac{v_{S}}{v_{T}}=\sqrt{\frac{r_{T}}{r_{S}}} \approx\left(C_{1}+C_{2}\right)^{n-C_{1}} * C_{2}^{C_{2}-n}
$$

Orbital velocity of Earth's space is $29.78 \mathrm{~km} / \mathrm{s}$. Average velocity of the event horizon for Earth is $2 / 3$ of this velocity, while the average velocity of time dimension is $1 / 3$ of this velocity:

$$
\begin{gathered}
v_{E H_{A V G}}=\frac{2}{3} 29.78=19.85333^{\prime} \mathrm{km} / \mathrm{s} \\
v_{T_{A V G}}=c_{t_{1}}=\frac{1}{3} 29.78=9.92666^{\prime} \mathrm{km} / \mathrm{s}
\end{gathered}
$$

Orbital radius of the time dimension is the space dimension of Saturn - Earth's time dimension is entangled with the space dimension of Saturn (time dimension of Saturn is entangled with Earth space dimension).

Average event horizon is entangled with the current orbit of Vesta, the dwarf planet.

Deviation of $\mathbf{v}_{\mathbf{T}_{\mathbf{A V G}}}$ from current Saturn orbit is equal to deviation of $\mathbf{v}_{\mathbf{E}} \mathbf{H}_{\mathrm{AVG}}$ from current Vesta: 


$$
v_{V e s t a}=\frac{v_{\text {Saturn }}}{v_{T_{A V G}}} * v_{E H_{A V G}}=3 * \frac{9.68}{29.78} * \frac{2}{3} 29.78=9.68 * 2=19.36 \mathrm{~km} / \mathrm{s}
$$

Speed of time for humans $\left(\mathbf{c}_{\mathbf{t}_{\mathbf{0}}}\right)$ is equal to standard speed of light $\mathbf{c}$, given the average life expectancy of 50 years $\left(2 * 10^{9}\right.$ heartbeats with $76 \mathrm{bpm}$ heart rate), the $3 \mathrm{rd}$ order period of Earth's existence cycle is:

$$
T_{x}=\frac{c_{t_{0}}}{c_{t_{1}}} * 50 \text { years }=3 * \frac{2.99792458 * 10^{8}}{29.78 * 10^{3}} * 50 \text { years }=1.51 * 10^{6} \text { years }
$$

\subsection{Body mass}

I have previously calculated initial (formative) real mass of Earth ${ }^{26}$ (as a particle) to be $2.93676{ }^{*} \mathbf{1 0}^{19}$ $\mathrm{kg}$.

If Earth is a living organism, predicting real mass of Earth in the same way as it is done with other organisms should give the result of the same order of magnitude (it likely won't be of equal value as Earth is evolving, gaining and loosing mass in the process).

Supposing that Earth is a mammal (or evolved from mammal), given the 3rd order existence half-life (period) $T_{x}$ of $1.512 * 10^{6}$ years, mass can be calculated from empirical relationship between mass and lifespan of mammalian species.

$$
\left(\frac{m_{E}}{m}\right)^{\frac{1}{4}} * T_{x_{M}}=T_{x}
$$

Given human adult mass $\mathbf{m}$ of $\mathbf{8 4} \mathrm{kg}$ and lifespan $\mathbf{T}_{\mathrm{X}_{\mathbf{M}}}$ of $\mathbf{5 0}$ years, mass of earth $\mathrm{m}_{\mathrm{E}}$ is:

$$
\begin{gathered}
m_{E}=m\left(\frac{T_{x}}{T_{x_{M}}}\right)^{4} \\
m_{E}=7 * 10^{19} \mathrm{~kg}
\end{gathered}
$$

Note that the value of $\mathrm{T}_{\mathrm{x}}^{4}, 5.2 * 10^{24}$ is roughly the value of the total gravitational mass of Earth $(\mathrm{M}=$ $\left.5.9723 * 10^{24}\right)$.

The result can be further verified by the previously established (in CR) equation for real mass:

$$
m_{E}=m_{r e}=\left(1-\sqrt{1-\frac{v_{r e}^{2}}{c_{s}^{2}}}\right) m_{i m g}
$$


where

$$
\begin{gathered}
v_{r e}=\frac{2 \pi r_{r e}}{T_{r e}}=\frac{2 \pi r_{s}}{T_{r e}} \\
c_{s}=\sqrt{\frac{G m_{i m g}}{r_{s}}} \approx \sqrt{\frac{G M}{r_{s}}}
\end{gathered}
$$

Using $T_{\mathrm{re}}=23.9^{*} 60^{*} 60=86040 \mathrm{~s}, \mathbf{G}=\mathbf{G}_{0}=6.673899 * 10^{-11} \mathrm{~m}^{3} / \mathrm{kgs}^{2}, \mathrm{r}_{\mathrm{s}}=1206115 \mathrm{~m}, \mathrm{~m}_{\mathrm{img}} \approx M=$ $5.9723 * 10^{24} \mathrm{~kg}:$

$$
m_{E}=7 * 10^{19} \mathrm{~kg}
$$

The results agree and show that Earth has gained roughly double the mass it had at the point of inflation/deflation of the gravitational maximum.

One might argue that it is impossible for Earth to have such low mass, as there is $\sim \mathbf{1 0}^{\mathbf{1 8}} \mathrm{kg}$ in surface oceans alone, $\sim 10^{22} \mathrm{~kg}$ in the crust, $\sim 10^{23} \mathrm{~kg}$ in the inner core and more in the mantle (based on density inferred from seismic profiles), however, these values are relative to the gravitational constant of the standard $\left(\mathbf{U}_{0}\right)$ scale $\mathbf{G}_{0}\left(6.674 * 10^{-11} \mathrm{~m}^{3} / \mathrm{kgs}^{2}\right)$.

Proper relativistic (effective) mass of Earth on $U_{1}$ scale is relative to $G_{1}\left(5.731534632 * 10^{-6} \mathrm{~m}^{3} / \mathrm{kgs}^{2}\right)$.

Proper equation for relationship between mass and lifespan is thus:

$$
G_{1} m_{E}=G_{0} m\left(\frac{T_{x}}{T_{x_{M}}}\right)^{4}
$$

Various results can now be obtained, depending on the value of the variables, as shown in Fig. 1.

Note, however, that obtained mass can also be interpreted as current imaginary mass, rather than real mass (in that case, real mass $=5.9723 * 10^{24} \mathrm{~kg}-7 * 10^{19} \mathrm{~kg} \approx 5.9723 * 10^{24} \mathrm{~kg}$ ) - as the gravitational well of a maximum acquires mass, this mass is shielding (replacing) the gravity of the maximum, so the obtained mass represents the remaining capacity for real mass (current img part of total gravitational mass), rather than current real mass.

\begin{tabular}{cllll}
$\mathrm{n}$ & $\mathrm{G}_{1}\left[\mathrm{~m}^{3} / \mathrm{kgs}^{2}\right]$ & $\mathrm{G}_{0}\left[\mathrm{~m}^{3} / \mathrm{kgs}^{2}\right]$ & $\mathrm{T}_{\mathrm{x}}$ & $\mathrm{m}_{\mathrm{E}}(\mathrm{n})[\mathrm{kg}]$ \\
\hline 1 & $5.731534632 * 10^{-6}$ & $6.674 * 10^{-11}$ & $25.82 \mathrm{My}$ & $6.9543 * 10^{19}$ \\
\hline 2 & $6.674 * 10^{-11}$ & $6.674 * 10^{-11}$ & $1.512 \mathrm{My}$ & $7.0244 * 10^{19}$ \\
\hdashline 3 & $6.674 * 10^{-11}$ & $6.674 * 10^{-11}$ & $25.82 \mathrm{My}$ & $5.9723 * 10^{24}$
\end{tabular}




\begin{tabular}{ccccc}
4 & $6.674 * 10^{-11}$ & $5.731534632 * 10^{-6}$ & $1.512 \mathrm{My}$ & $7.1816 * 10^{22}$ \\
\hline 5 & $6.674 * 10^{-11}$ & $5.731534632 * 10^{-6}$ & $25.82 \mathrm{My}$ & $5.1290 * 10^{29}$ \\
\hline 6 & $6.674 * 10^{-11}$ & $6.674 * 10^{-11}$ & $19.3 \mathrm{~s}$ & $1.8802 * 10^{-30}$ \\
\hline 7 & $4.9000394 * 10^{-2}$ & $6.674 * 10^{-11}$ & $4.25 \mathrm{~Gy}$ & $5.9723 * 10^{24}$
\end{tabular}

Fig. 1: Relative Earth mass

Here, $m_{E}(\mathbf{1})$ is the proper relativistic mass of Earth calculated with 2 nd order $T_{X}, m_{E}(2)$ is the proper relativistic mass calculated using $3 r d$ order $\mathbf{T}_{\mathrm{X}}$. Third mass, $\mathrm{m}_{\mathrm{E}}(\mathbf{3})$, is the mass of Earth relative to standard scale $\left(\mathbf{m}_{\mathrm{E}_{0}}\right)$ calculated using $2 \mathrm{nd}$ order $\mathbf{T}_{\mathrm{X}}$.

Masses $\mathrm{m}_{\mathrm{E}}(\mathbf{4})$ and $\mathrm{m}_{\mathrm{E}}(\mathbf{5})$ could be considered as inverse (or anti) masses of Earth relative to its [past] event horizon (inner core maximum).

Note that $m_{E}(4)$ is [roughly?] equal to $2 / 3$ of the mass of the Earth's inner core, while $m_{E}(5)$ is roughly $1 / 4$ of the Sun's mass.

Note also the presence of multiple periods in the cycling of Earth's [maximum] existence, 1.512 My and 25.82 My. While the shorter period could be considered as a fossil of the Solar System $U_{0}$ half-life $\left({ }^{10} \mathrm{Be}_{0}\right)$, this entanglement cannot be lost completely and some time compression at the end of $1.512 \mathrm{My}$ cycles can also be expected.

While the periods of $2 \mathrm{nd}$ and $3 \mathrm{rd}$ order represent the half-life of Earth's gravitational maximum quanta, these do not represent the lifespan of Earth.

At the end of these cycles, the major maximum only temporarily collapses (partially, in time and space), proportionally to the cycle period. If the maximum is interpreted as a soul, which I consider the correct interpretation, such collapse is a temporary loss of consciousness.

I have previously hypothesized that the Solar System is the product of annihilation and inflation of ${ }^{10} \mathrm{C}$ and ${ }^{10} \mathrm{Be}$ atoms of smaller scale, thus, the entanglement with ${ }^{10} \mathrm{C}$ can also be expected, although the collapse and the induced time (evolution) compression should be negligible due to short half-life (19.3 s) of ${ }^{10} \mathrm{C}$.

Note that Earth is in $2 e$ configuration, and with $T_{X}$ of $19.3 \mathrm{~s}$, mass of Earth $\left[\mathrm{m}_{\mathrm{E}}(\mathbf{6})\right.$ ] becomes roughly equal to the mass of 2 standard electrons (or positrons).

Also note that the initial real mass of Earth $\left(2.93676 * 10^{19} \mathrm{~kg}\right)$ is roughly half of $\mathrm{m}_{\mathrm{E}}(1)$ and should correspond to 1e configuration. 
If $\mathrm{m}_{\mathrm{E}}(\mathbf{4})$ and $\mathrm{m}_{\mathrm{E}}(\mathbf{5})$ are correlated with Earth's inner core and Sun mass, the data suggests asymmetry between mass and inverse mass, growing with period $\mathbf{T}_{\mathrm{X}}$.

The solution is the inflation of $\mathbf{T}_{\mathrm{x}}$ and/or $\mathbf{G}$.

With $\mathbf{G}_{0}$ [roughly] equal to $2.222 * 10^{-5} \mathrm{~m}^{3} / \mathrm{kgs}^{2}, \mathrm{~m}_{\mathrm{E}}(\mathbf{5})$ becomes equal to the mass of the Sun, while for $G_{0}$ [roughly] equal to $1.9561 * 10^{-5}, m_{E}(4)$ becomes equal to to the proper relativistic mass of the Sun.

The same can be obtained with $\mathrm{T}_{\mathrm{x}}$ equal to $36.23 \mathrm{My}$ and 2.06 My, respectively.

With a period of $\mathbf{5 5 5 6 1 9 . 1 1}$ years, $\mathrm{m}_{\mathrm{E}}(\mathbf{4})$ becomes equal to inner core mass (assuming that mass is $\left.1.1 * 10^{23} \mathrm{~kg}\right)$.

Interestingly, for $T_{x}$ equal to the 1st order period (4.25 Gy), the result of equation M1.1, rounded to 2 decimals, is equal to speed of light on $U_{1}$ scale $\left(2.93 * 10^{6} \mathrm{~m} / \mathrm{s}\right)$ multiplied by $10^{17}$.

Note also that the ratio between $\mathbf{G}_{\mathbf{1}}(\mathbf{7})$ and $\mathbf{G}_{\mathbf{1}}(\mathbf{1})$ is roughly equal to ratio between $\mathbf{G}_{\mathbf{1}}(\mathbf{1})$ and $\mathbf{G}_{\mathbf{0}}(\mathbf{1})$ divided by 10:

$$
G_{1}(7) \approx \frac{1}{10} \frac{G_{1}(1)}{G_{0}(1)} G_{1}(1)
$$

which is consistent with association of different G's to different vertical energy levels and therefor to scale (period) of general oscillation.

If $\mathbf{G}_{\mathbf{0}}(\mathbf{1})$ would, as hypothesized previously, belong to $\mathbf{U}_{\mathbf{0}}$ scale, $\mathbf{G 1}(\mathbf{1})$ should be associated with $\mathbf{U}_{\mathbf{1}}$ scale and $\mathbf{G 1}(7)$ with $\mathrm{U}_{2}$ scale.

If one assumes that:

$$
G_{1}(7)=\frac{1}{10} \frac{G_{1}(1)}{G_{0}(1)} G_{1}(1)
$$

one obtains a $T_{X}$ of the 1 st order of $4.254788 \mathrm{~Gy}\left(4.254788 * 10^{9}\right.$ years $)$.

\subsection{Future development}


Current acidification of oceans and drinking water was triggered by homo.beta through fossil fuel extraction and burning.

This may be interpreted as a signal that homo.beta, being part of progenitor cells in planetary neurogenesis, is ready to transform.

Homo.beta refers to species of humans currently inhabiting the Earth's surface 27.

At the time homo.beta becomes aware of this the point of no return may generally be crossed already as a normal part in evolution of an Earth-like species (homo.omega).

The acidification is only part of global changes with the aim of formation of new brain tissue of homo.omega.

After the initial pulse, the process is further managed by the Solar System, which includes relative cataclysmic changes:

- increasing rate of volcanism and earthquakes,

- burning of fossil-fuel reserves,

- gyrification of brain tissue (mantle layers),

- asteroid impacts,

- extreme weathering,

- sea level changes (melting of all polar ice).

Here I hypothesize that the ocean $\mathbf{p H}$ of $\mathbf{7 . 3 3}$, being equal to the $\mathbf{p H}$ of cerebrospinal fluid (CSF), is a significant [turning] point and current acidification of oceans will continue accelerating until $\mathbf{p H}$ drops to this value. A that point, [properly scaled] surface cells/proteins should migrate to mantle.

Based on correlation with atmospheric $\mathrm{CO}_{2}$, climate models predict this $\mathrm{pH}$ minimum in the year $2300 \mathrm{AD}$ for an atmospheric concentration of $\mathrm{CO}_{2}$ of $1900 \mathrm{ppmv}^{28}$ (all fossil-fuel sources burned).

The 7.33 $\mathrm{pH}$ and associated $\mathbf{C O}_{2}$ level have been reached before on Earth - in mass extinction events such as Permo-Triassic ${ }^{29}$.

These mass extinction events are events of strong, accelerated evolution - specifically, layer formation events of Earth's brain [mantle], which includes differentiation of progenitor cells at the surface. 
Although some species get extinct at the event, most strongly evolve to survive in more acidic and underground environment, where they are transferred together with the acidified ocean.

Afterwards, new surface water is delivered by asteroid (cometary) impacts (not at random locations).

Due to Solar System carbon nature $(6 \mathrm{p} 6 \mathrm{e})$, homo brain has 6 neocortex layers and, possibly due to Earth's 2e configuration, 2 hemispheres.

A precursor of 6 layers has been created in events during Precambrian era, while population with neuron cells and final formation is occurring in Phanerozoic.

There have been 5 major extinctions in Phanerozoic, thus the next event should populate top layers and complete the formation of the final layer (I):

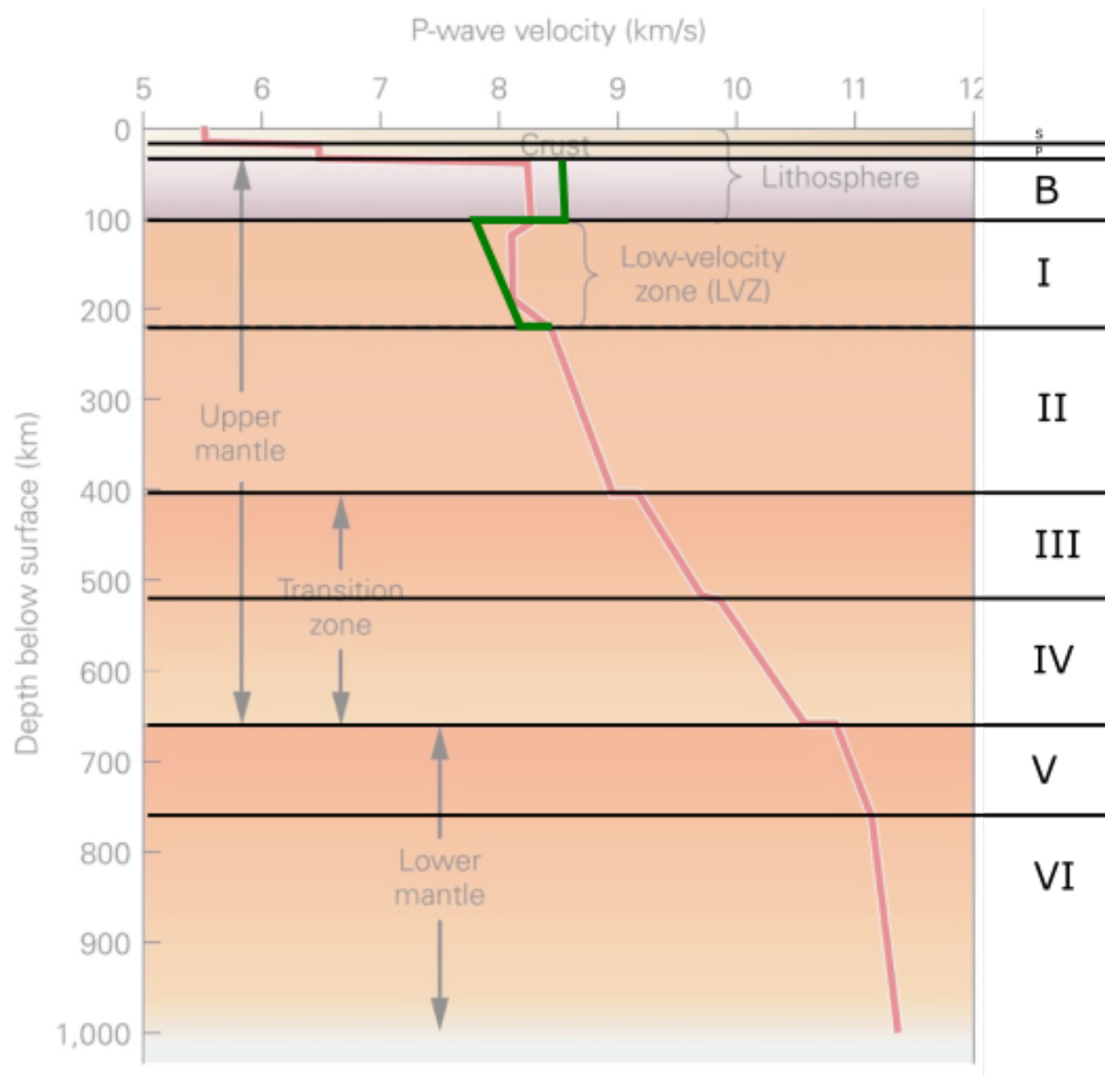

(a) The velocity of P-waves changes with depth in the mantle.

Fig. 2: Layers of Earth's brain, superimposed on seismic velocities ${ }^{30}$

Formed layers of Earth's brain are shown on Fig. 2. It is evident that layer I is yet to complete formation green line shows possible seismic velocities after formation.

Energy from the Sun provides incubation energy used for the maintenance of the Earth's surface 
ecosystem and slow evolution, but additional energy is needed for the formation of brain layers of omega.

This energy, in case of homo.omega is delivered through asteroid (food) and cometary (water) impacts.

Year $2300 \mathrm{AD}$ for the event is very conservative though, as it is based on linear extrapolation, does not include rising water temperatures and reaction of the biosphere.

Acidification of water at these events must be, in significant part, driven by injections of gases through oceanic ridges and vents which would introduce significant departure from linear correlation of $\mathbf{p H}$ with atmospheric $\mathrm{CO}_{2}$.

Mathematical analysis of past extinctions ${ }^{31}$ also suggests sooner triggering of 6 th major extinction event, by the year $2100^{32}$.

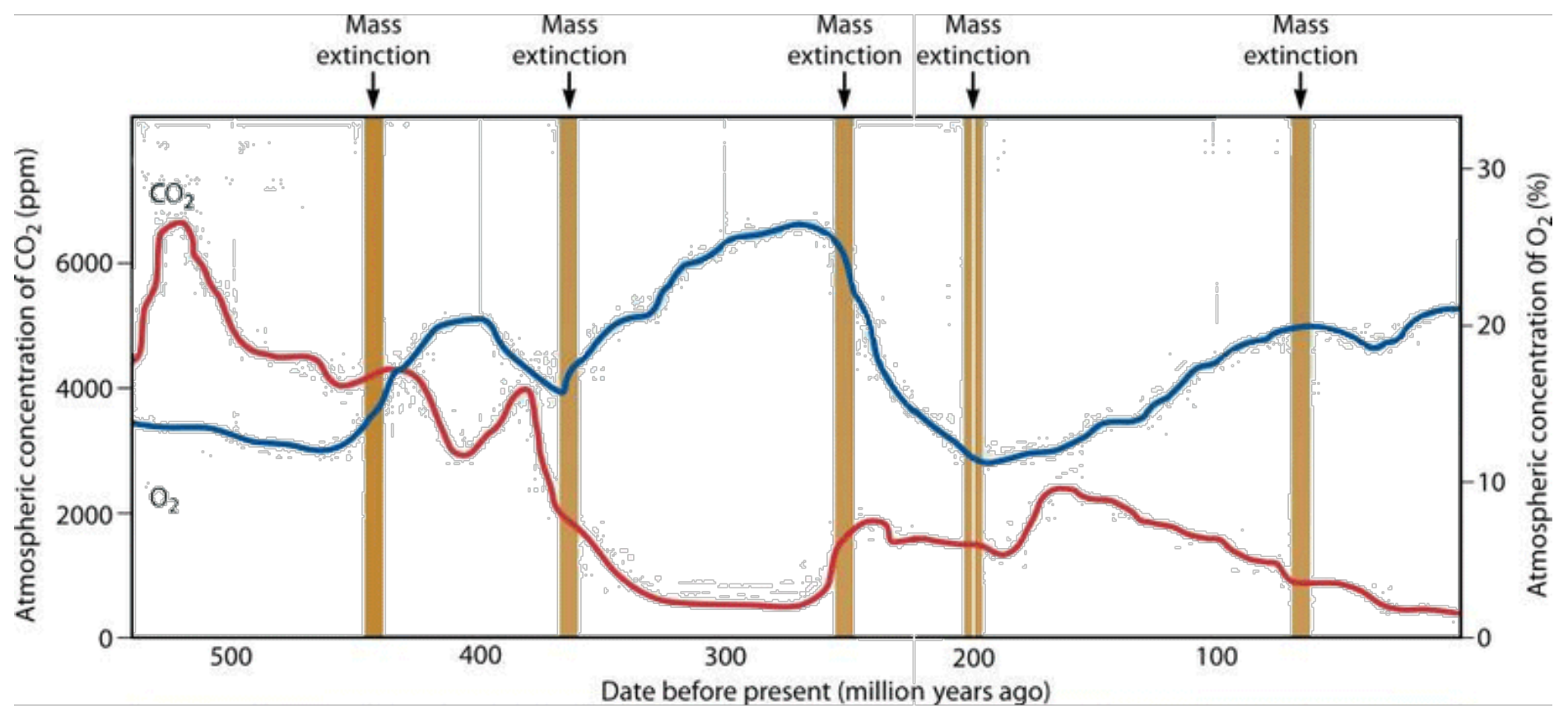

Fig. 3: The history of atmospheric $\mathrm{CO}_{\mathbf{2}}$ concentration ${ }^{33}$

From Fig. 3 and more recent models ${ }^{34}$, it is evident that $\mathbf{C O}_{2}$ concentration has a decreasing trend (expected due to increased energy from the Sun = less greenhouse gases needed to maintain the temperature).

Everything in nature oscillates (and fluctuates) so this decrease in amplitude should not be linear either, due to scale invariance and self-similarity of universes, the oscillatory pattern of $\mathbf{C O}_{2}$ at mass extinction events should resemble carbon cycles of homo embryonic development.

In a quantum system, it would be reasonable to expect markers of extinction (formation) events as products of harmonic oscillation. A plausible physical interpretation of such markers are asteroid impacts. 
Statistically significant periodicity of extinctions ${ }^{35}$ (at least in the last $\mathbf{2 5 0}$ million years) has been noted before - 26, and more recently, 27 million years between extinctions ${ }^{36}$. In any case, due to differences in extinction strength, multiple harmonics (or energy splitting of a single oscillator) are possible.

Using available data, one can construct some plausible models:

\begin{tabular}{|c|l|l|l|l|l|}
\hline year [mya] & a) $\mathrm{CO}_{2}[\mathrm{ppm}]$ & b) $\mathrm{CO}_{2}[\mathrm{ppm}]$ & c) $C \mathrm{O}_{2}[\mathrm{ppm}]$ & d) $\mathrm{CO}_{2}[\mathrm{ppm}]$ & e) $C \mathrm{O}_{2}[\mathrm{ppm}]$ \\
\hline 444 & 3800 & 200 & 2000 & 3800 & 2000 \\
\hline 370 & 1000 & 2000 & 1000 & 1800 & 1200 \\
\hline 252 & 800 & 900 & 800 & 800 & 800 \\
\hline 201 & 1800 & 1800 & 1800 & 1800 & 600 \\
\hline 66 & 250 & 250 & 250 & 300 & 500 \\
\hline 0 & 450 & 700 & 750 & 800 & 450 \\
\hline
\end{tabular}

Table. 1: $\mathrm{CO}_{2}$ impact trigger models 
a)

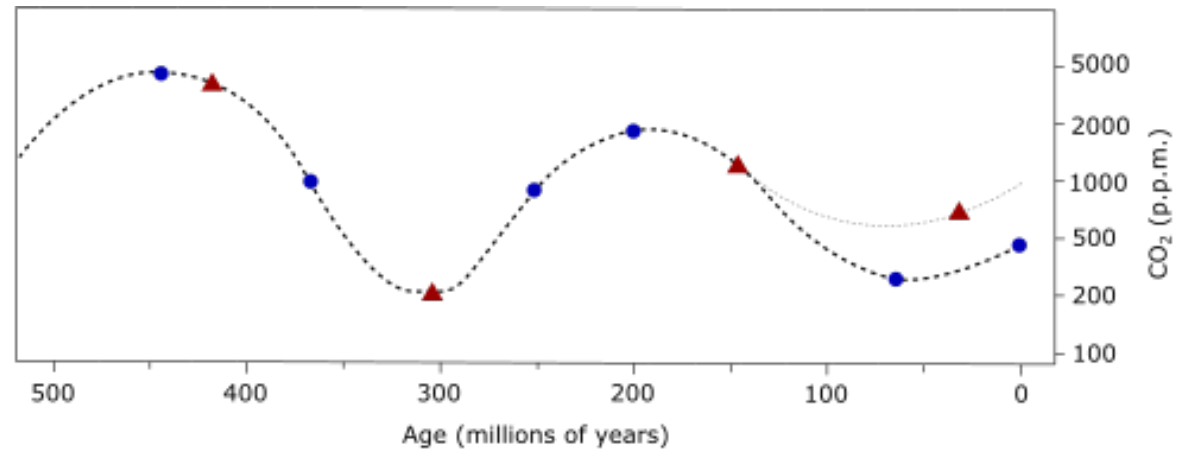

b)

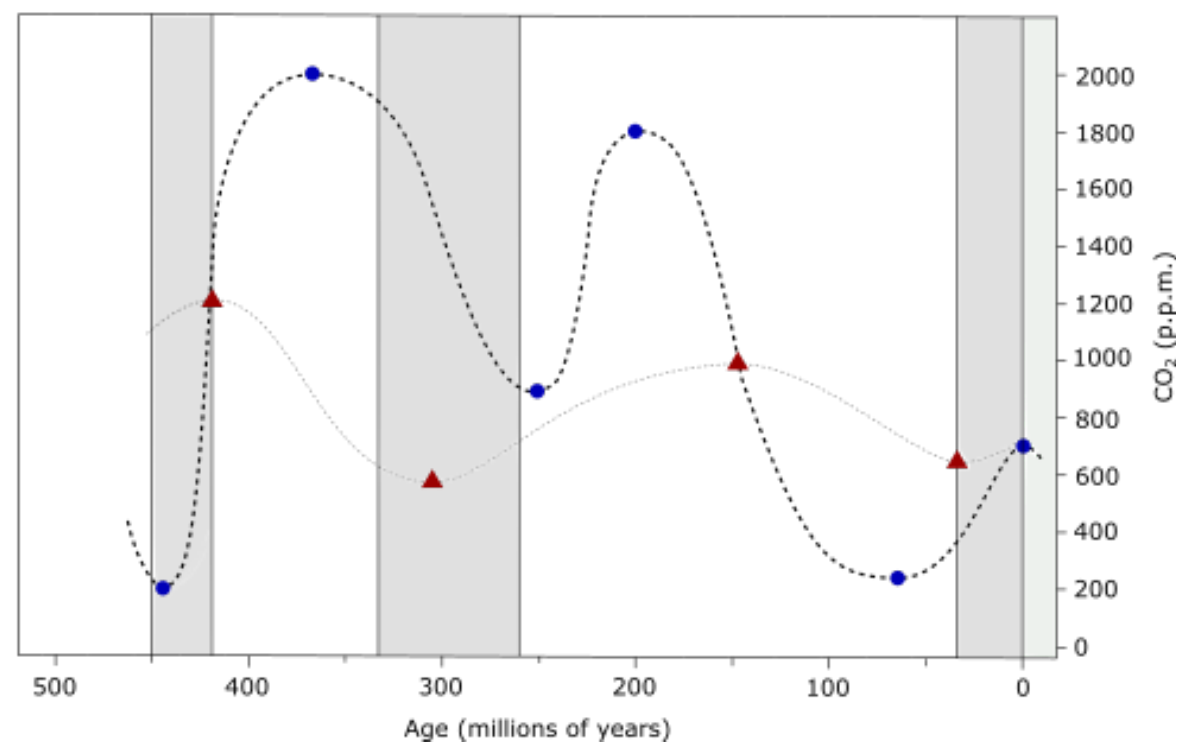

Fig. 4: $\mathrm{CO}_{2}$ models a) and b) (blue dots = major extinction events, red = minor extinction events, on b) grey = icehouse periods, white $=$ greenhouse periods)

Models are constructed in such a way to simulate oscillation of markers and compression of the amplitude with time, but they are also quantized - each marker is a multiple of $\mathbf{5 0 ~ p p m ~} \mathrm{CO}_{2}$ quantum.

Some of the models are shown on Fig. 4, blue dots are major extinction events, red triangles are minor extinction events (the curve does not necessarily follow actual $\mathrm{CO}_{2}$ levels between the extinctions, it is only used to illustrate oscillation).

There is some apparent grouping of extinctions, suggesting oscillation of frequency. Major extinctions can be grouped into pairs separated by $126.5( \pm 8.5)$ million years, while paired extinctions are separated by roughly half that distance - $62.5( \pm 11.5)$ million years. Minor extinctions $(420,305,145 \& 34$ mya) may be grouped in the same way - pairs separated by 160 million years, $113( \pm 2)$ million years separation of paired extinctions.

Model a) is the product of energy level splitting of a single oscillator, while $\mathbf{b}$ ) is the product of $\mathbf{2}$ harmonic oscillators - one high energy (major) and one low energy (minor).

The points on the curve are not maximum $\mathrm{CO}_{2}$ levels during the extinction and should be interpreted as 
points of mass extinction triggers (asteroid impacts).

According to above models, the maximum atmospheric $\mathrm{CO}_{2}$ concentration for such event in this extinction (pH 7.33) is thus 800 ppmv.

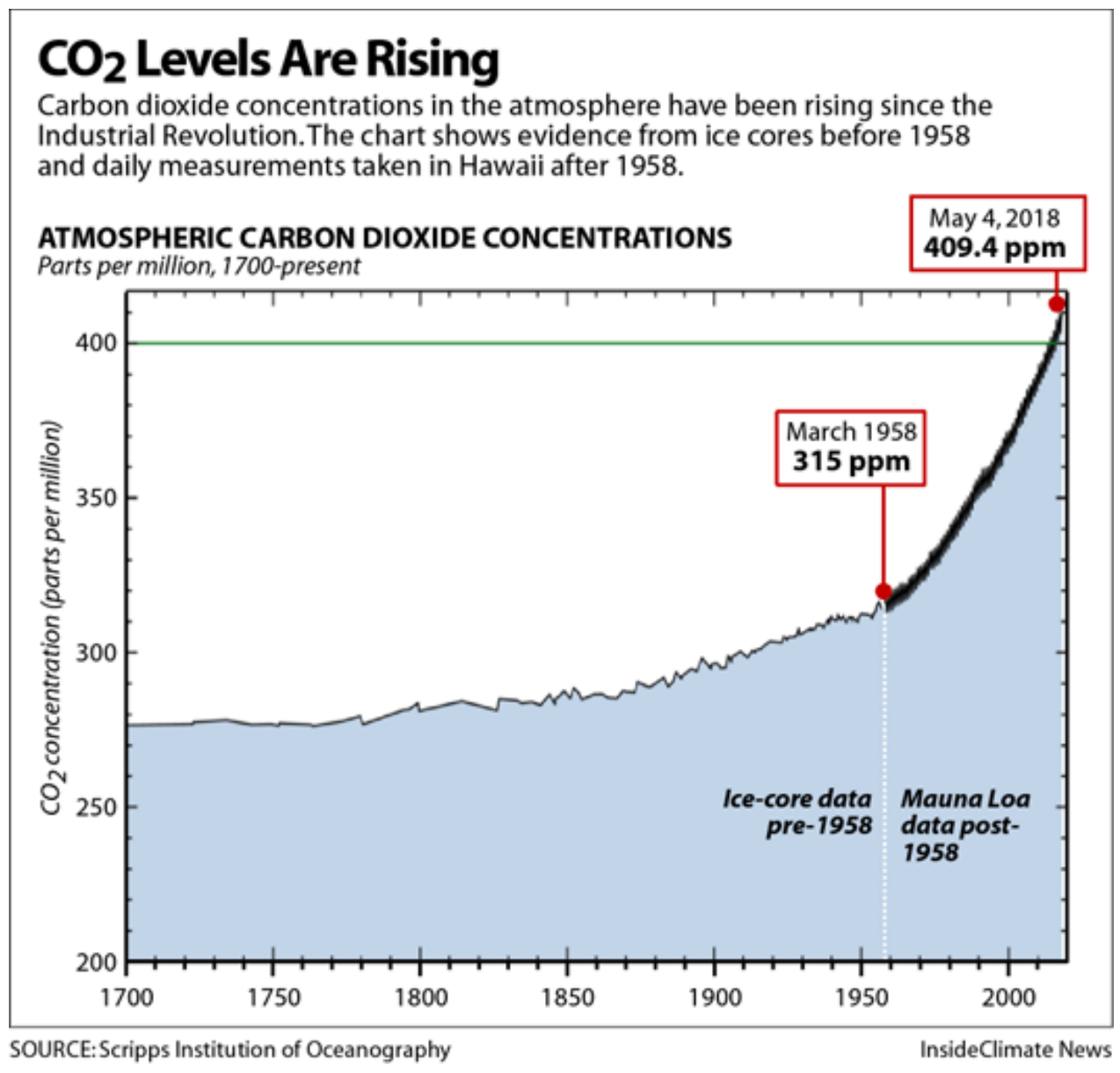

Fig. 5: Recent history of $\mathrm{CO}_{2}$ concentration ${ }^{37}$

Extrapolating from Fig. 5, one can obtain this relation for $\mathrm{CO}_{2}$ concentration, from year 1850 onward:

$$
\begin{gathered}
C O_{2}=300 *\left(\frac{6}{5} * 2^{45 x^{2}}\right)^{x} \mathrm{ppmv} \\
x=\frac{T-1905}{10 * 55}=\frac{T-1905}{550}
\end{gathered}
$$

which, for the concentration of $\mathbf{8 0 0}$ ppmv gives year $\mathbf{T}=\mathbf{2 0 7 5}$. This is the best case scenario (for those not looking forward to the event).

\begin{tabular}{|l|l|l|l|l|l|}
\hline model & $\begin{array}{l}\mathrm{CO}_{2} \\
{[\mathrm{ppm}]}\end{array}$ & $\begin{array}{l}\text { date of impact } \\
\text { [year] }\end{array}$ & $\begin{array}{l}\text { associated impactor } \\
\text { (diameter) }\end{array}$ & $\begin{array}{l}\text { impactor closest } \\
\text { approaches }\end{array}$ & $\begin{array}{l}\text { 2nd order impactor } \\
\text { (diameter) }\end{array}$ \\
\hline a), e) & 450 & 2029 & 99942 Apophis $(\approx 375 \mathrm{~m})$ & $\mathbf{2 0 2 9 , 2 0 6 5}$ \\
\hline
\end{tabular}




\begin{tabular}{|l|l|l|l|l|l|} 
b) & 700 & 2066 & 99942 Apophis $(\approx 375 \mathrm{~m})$ & 2029,2065 & \\
\hline c) & 750 & 2071 & 1866 Sisyphus $(\approx 7 \mathrm{~km})$ & 2071 & 2000 SG $_{344}(37 \mathrm{~m})$ \\
\hline d) & 800 & 2075 & 162173 Ryugu $(\approx 1 \mathrm{~km})$ & 2076 &
\end{tabular}

Table. 2: Calculated dates for $\mathrm{CO}_{2}$ impact trigger models

Table 2. shows calculated dates and probable impactors (possible 2nd order impacts are low energy impacts).

Evidently, for all of these simple oscillatory models, there are clear candidates among extinction causing asteroids in NEO (near Earth orbit).

A realistic time frame for asteroid impacts, for a $\mathbf{C O}_{2}$ marker maximum of $\mathbf{8 0 0}$ ppm is thus 2029 - 2075 .

Multiple extinction pulses are also plausible (ie. one in 2029 and other in 2066) - due to splitting of energy levels (breaking of Apophis - homo induced?), as has most likely happened in previous extinctions.

Note 1: According to current models based on Newtonian mechanics, none of these asteroids is on a collision course with Earth in near future.

However, these models do not predict periodic existence/extinction pulses coupling with a collapse of gravitational maximums.

As part of $6 \mathrm{p} 4 \mathrm{n} / 4 \mathrm{p} 6 \mathrm{n}$ oscillation, a temporary inflation of a gravitational maximum is expected at $3 / 4$ MAU (1.14 AU) distance from the Sun - considering the orbits of these asteroids, a gravitational well at this distance with enough energy could certainly cause orbital precession, sending the impactor on a collision course with Earth.

However, changes in the Earth/Moon system are also expected, and these too can affect orbitals of these asteroids. Ejecta from the Sun can also alter the course of an asteroid, as well as passing of the Solar System through extrasolar asteroid field.

Since these impacts are DNA coded, they should not be questionable, it is only the source and method of delivery that may be unknown prior to the event.

Note 2: Interestingly, there was an impact event on Earth at the time when $400 \mathrm{ppm} \mathbf{C O}_{2}$ was reached (Chelyabinsk meteor, $\approx 20$ m diameter, 2013.).

Timing of this event compared with models even suggests periodic bombardment with each $\mathbf{5 0}$ ppm 
quantum of $\mathrm{CO}_{2}$.

After all, Solar System is a quantum system, and this would be the effect of superposition of states (harmonics) in a quantum of time.

Even though the Chelyabinsk event occurred in the year when $\mathbf{4 0 0}$ ppm was first reached, I believe the events should be correlated with the average ppm value given by the $\mathbf{C} 1.1$ equation.

For 400 ppm, the equation gives year 2015 (one may thus assume the events occur at $T \pm n$ years, where $\mathbf{n}$ is time dependent, decreasing by half with every 100 ppm - being equal to 4 at 300 ppm, 2 at 400 ppm, etc.). Assuming the correlation of events with $\mathbf{C O}_{2}$ increase beyond background levels, the first event should have occurred at $\mathbf{3 0 0}$ ppm - the beginning of industrial revolution. One such event did occur at $\mathbf{3 0 0}$ ppm - Tunguska, 1908.

The equation gives year 1992 for 350 ppm. No meteors of comparable impact energy were recorded in or around 1992., eliminating direct impacts on land area. If such event did occur, it must have occurred over the ocean (or island), triggering large waves and possibly earthquakes. Interestingly, an 7.2+ magnitude earthquake and tsunami wave did occur offshore in Nicaragua - in 1992.

This earthquake is notable for tsunami wave being unusually large $(9.9 \mathrm{~m}$ high) for the strength of the earthquake (belonging to a group of rare tsunami earthquakes).

I do not believe, however, that the impact caused the earthquake. This was likely the effect of synchronization of events (synchronicity) - the tsunami was caused by the earthquake but it was amplified by the impact. The Earth is a living being and it would not be surprising it reacts to incoming bolides and impactors (just like humans do).

I have witnessed such synchronization myself - on 2019.03.07 I have observed a larger meteor burning up in the atmosphere exactly at the time of earthquake in Hungary, it's trajectory was toward the epicenter. It is even possible that Earth reacts to every possible impactor, although the reaction may be proportional to impactor energy and thus usually negligible.

Also interesting about the Nicaragua event is that it occurred at the time of my birthday (September 1st, local time) producing an obvious signal ${ }^{38}$ for me. I interpret this as a confirmation that the meteor was involved in this event.

Note that such sign[al]s are not predicted by QM or GR and synchronicity at the point of writing of this article was still not considered real by modern science. However, with $\mathbf{C R}$ the phenomena becomes not only real, but a [relatively] special form of synchronization and a driver of evolution with exponentially increasing significance near the end of an existence cycle. 
Thus, if one doesn't believe in signals of synchronicity (I didn't before CR) I suggest one to study all my work, particularly the work referenced above.

Note that Nicaragua, Chelyabinsk and Tunguska impact sites on the world map can be connected with a straight line - a correlation suggesting that next impact may also occur somewhere along this line (even the Chicxulub, Yucatan crater is close).

Although there were no sightings of large meteors over land, a smaller magnitude impact was recorded on land area in 1992 - the Peekskill meteorite. It was recorded one month after the Nicaragua event and is notable for hitting a car in urban area.

Also interesting, and symbolic, is a fact that the last visit of the Halley's comet to the inner Solar

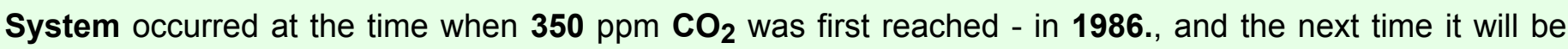
close to Earth is 2061 . - exactly at 650 ppm (calculated using the C1.1 equation).

It is currently hypothesized that Tunguska event was caused by a large body which eventually escaped Earth's atmosphere - it can thus be interpreted as a warning.

Given the fact that neither the Chelyabinsk nor hypothesized Nicaragua meteor did not directly impact land, it appears these too were warnings.

However, I do not interpret these as warnings, I believe one purpose of the atmosphere is to disintegrate incoming bodies to protect life during weak evolution. Thus, much larger bodies and impacts should be expected only as events synchronized with strong evolution.

These recent events may thus be interpreted as signals of things to come. After these $\mathbf{3}$ misses, I believe next will be impacts, and, with the year 2029, one can expect the first wave of strong evolution, with the last wave occurring 2060. - 2084. or 2061. - 2085 (corresponding to 650 ppm and 950 ppm, respectively).

Note that Newton correctly calculated year 2060 as the beginning of the end of surface world, although he revised this year later to $\mathbf{2 0 1 6}$ by the suggestion of others. His final decision to revise the year was, however, based on a signal. As he was doing calculations, large earthquake occurred which he later interpreted as a signal that the year $\mathbf{2 0 6 0}$ is wrong. This earthquake was a signal, but he misinterpreted its meaning - large and frequent earthquakes are to be expected at the end.

The year 2016 is not there without a meaning for me though, it is the year of my soul rebirth (change of energy level) occurring at the age of $\mathbf{3 5}$ of the incarnation ${ }^{39}$. 
Note 3: Interestingly, at the time of the Chelyabinsk event, the Apophis asteroid was in close approach. Considering that the composition of Chelyabinsk meteor seems to match the composition of Apophis surface (LL chondrite) a probability exists that the meteor broke off of Apophis.

Note 4: All above confirms that the initially chosen $\mathbf{5 0}$ ppm quantum was good. I cannot attribute this to blind luck. The coherence of signals with my thoughts has been only growing ever since I've started experiencing them. Even prior to the models, based on intuition, I have felt years 2029 and 2066 as probable impact dates, and I wasn't even aware of Apophis at the time.

For me, the outcome of models and equations is often a confirmation of a signal rather than the other way around.

However, I will rarely mention signals [or use them as evidence] in my works, as I believe most of people do not sense or recognize them yet and even I could still misinterpret the signal. That is one of the reasons I use logic, equations and models to explain, analyze and predict phenomena. This, I consider necessary to fine-tune the coherence and signal interpretation.

Note 5: The equation C1.1 is one variant of a universal equation for a pulse of strong evolution. That 800 ppm as the $\mathbf{C O}_{2}$ marker maximum was a good prediction can be confirmed with another variant (inverse) of the equation, one correlated with half-lives of elements:

$$
\begin{gathered}
T_{1 / 2}=2 C_{1}-\frac{C_{1}}{C O_{2}\left(T_{1}\right)} * C O_{2}=2 C_{1}-\frac{C_{1}}{C O_{2}\left(T_{1}\right)} * 300 *\left(\frac{6}{5} * 2^{45 x^{2}}\right)^{x} \\
x=\frac{T-1905}{10 * 55}=\frac{T-1905}{550}
\end{gathered}
$$

where $C_{1}=T_{1 / 2}\left(T_{1}\right)$ is the half-life of the element measured at time $T_{1}$. The equation gives half-life of 0 at, or near, $\mathbf{T}=\mathbf{2 0 7 5}$, which is the year when $\mathbf{C O}_{2}(\mathrm{~T})$ is equal to $\mathbf{8 0 0} \mathrm{ppm}$. Just like in case of $\mathbf{C O}_{2} \mathbf{I}$ do not expect for half-lives to follow the equation continuously (ie. half-life might appear constant and then get reduced significantly in an instant). Generally, changes in decay rates require sudden structural changes of space.

One exception to this, in the Solar System, might be the half-life of ${ }^{10} \mathrm{Be}$, due to close scale entanglement. Solar System (scale $\left.U_{1}\right)$ cycles through ${ }^{10}(C-B-B e)$ and I would expect a continuous precursor enrichment in $10 \mathrm{~B}$ at a lower scale $\left(\mathrm{U}_{\mathbf{0}}\right)$ before the state change of $\mathrm{U}_{\mathbf{1}}$.

For ${ }^{10} \mathrm{Be}$, incorporating the value from the most recent measurements $\left(T_{1}=\mathbf{2 0 1 0}, T_{1 / 2}(2010)=1.387\right.$ $* 10^{6} \mathrm{y}$ ), the half-life equation is:

$$
T_{1 / 2}=2 * 1.387 * 10^{6}-\frac{1.387 * 10^{6}}{385.91546173092784386776172300903} * 300 *\left(\frac{6}{5} * 2^{45 x^{2}}\right)^{x}
$$


and it gives values in good agreement with previous measurements:

\begin{tabular}{|c|c|c|c|}
\hline year & calculated $\left[10^{6}\right.$ years] & sample & measured $\left[10^{6}\right.$ years] \\
\hline 1947 & 1.665 & & $1.7 \pm 0.4 * \dagger$ \\
\hline $1947(2)$ & 1.665 & & $1.6 \pm 0.2 * \dagger$ \\
\hline 1972 & 1.608 & & $1.5 \pm 0.3$ \\
\hline 1975 & 1.597 & & $1.48 \pm 0.15$ \\
\hline 1986 & 1.550 & NIST-4325 & $1.34 \pm 0.07$ \\
\hline 1987 & 1.545 & ORNL-MASTER & $1.51 \pm 0.06 \dagger$ \\
\hline 1993 & 1.513 & NIST-4325 & $1.53 \pm 5 \%(1.53 \pm 0.07) \dagger$ \\
\hline $1993(2)$ & 1.513 & $\mathrm{ICN}$ & $1.48 \pm 5 \%(1.48 \pm 0.06) \dagger$ \\
\hline 2007 & 1.413 & $\mathrm{ICN}$ & $1.36 \pm 0.07$ \\
\hline 2010 & 1.387 & & $1.388 \pm 0.018$ \\
\hline $2010(2)$ & 1.387 & & $1.386 \pm 0.016$ \\
\hline
\end{tabular}

Table 3: Calculation and measurements ${ }^{40}$ of ${ }^{10}$ Be half-life

* the value is not the initially published value, but the result of reanalysis/correction in 1972 ., $\dagger$ these values are discarded by scientific community, citing potential systematic errors (based on the presumption of absolute constancy of decay rates).

All measurements agree well with calculated values, except for 1986 - if there were no flaws in measurement, this may be attributed to deviation due to cycling (similar to yearly fluctuation of $\mathrm{CO}_{2}$ ). $\mathrm{Note}_{\text {, }}$ however, that measurement 1993 was done on the same SRM (Standard Reference Material) sample and discrepancy suggests one of these measurements is wrong.

If indeed the half-life of ${ }^{10} \mathrm{Be}$ is decreasing as hypothesized, modern science has been effectively doing cherry-picking here - discarding results which do not agree well, or are in discrepancy, with latest measurements.

Given the current precision of measurements, a new measurement at this point in time which would agree with the calculation would be in discrepancy with measurements from 2010. and would thus confirm the hypothesis of continuous decrease of ${ }^{10} \mathrm{Be}$ half-life prior to the extinction event.

Note that this effect on decay rates is temporary and significant only at the end of a cycle of general oscillation up to the 3 rd order. 


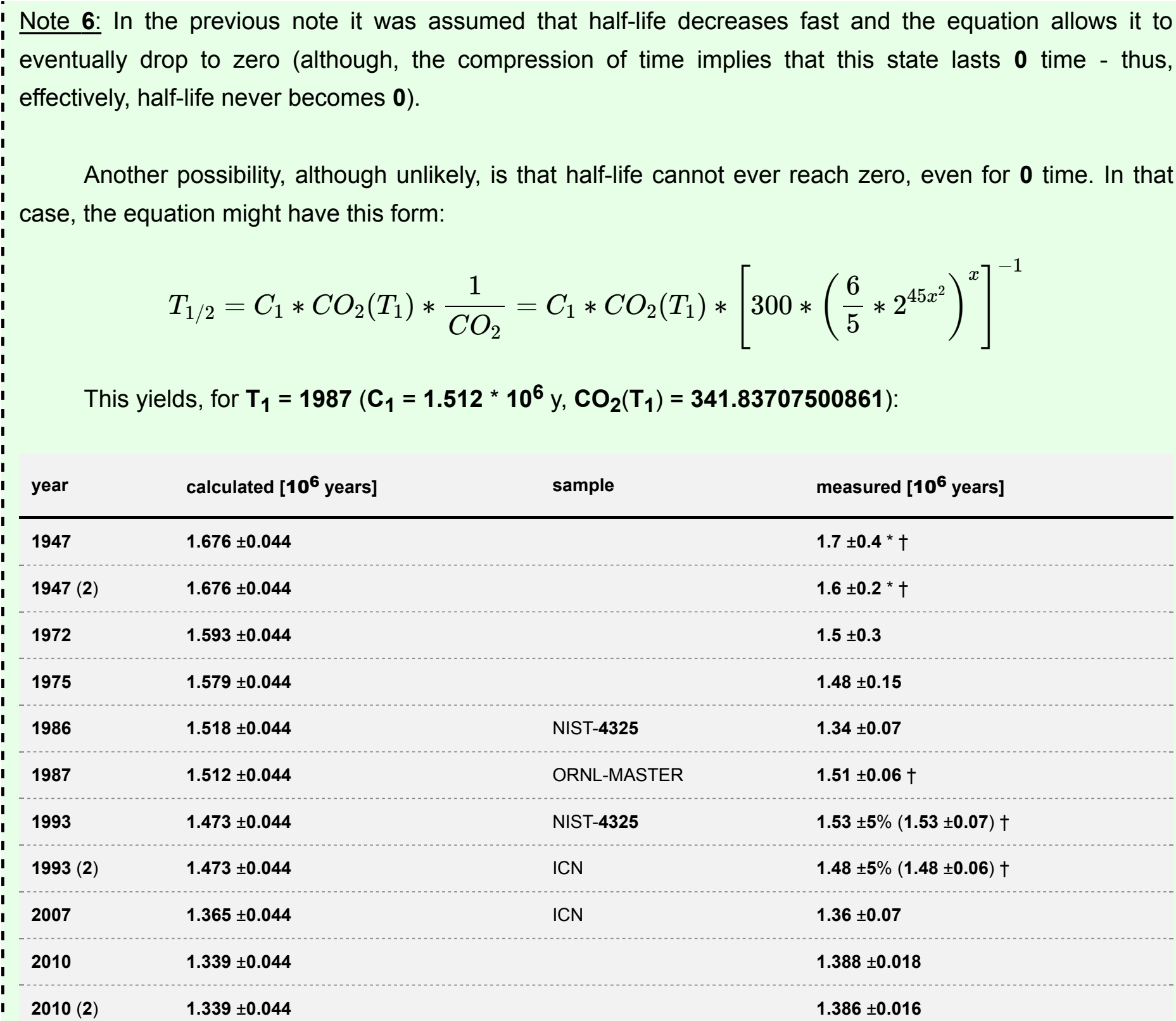

Table 4: Calculation and measurements of ${ }^{10}$ Be half-life

where error margin in calculation is the scaled variation of $\mathrm{CO}_{2}(\mathbf{1 0} \mathrm{ppm})$.

Such pulses might not only be plausible but necessary - first pulse would include asteroid impact(s) (to trigger ocean acidification and formation of the layer in the mantle), the other would be cometary (to provide new water/life). A third pulse in between might also be needed to trigger the (now acidified - CSF) ocean sink and, relatively, sterilize the surface.

However, considering that life forms on Earth can produce the acidification and trigger the extinction (as calculated by Rothman) a slightly different scenario might be more plausible: 
1. the Earth itself gives a signal (ie. through anthropogenic $\mathbf{C O}_{2}$ increase) that it is ready for next evolution step, acidification achieved through positive feedback,

2. asteroid impacts trigger, or coincide with, the gravitational well (incl. magnetic field) collapse and ocean (CSF) sink, sterilizing surface by UV/gamma radiation (no magnetic field),

3. cometary impacts deliver new water/life.

It might seem that a cometary impact this time is not needed - as layer formation should be complete with this extinction (corresponding to Carbon nature of the Solar System) there is no need for cultivation of new progenitor cells on surface. However, it probably does happen as it would provide additional radiation protection and provide support for whatever life remains on surface.

Note that a collapse of Moon's gravitational maximum is expected, remains of the Moon are thus the most likely source of eventual impacts of cometary nature (dust/water/ice).

This is evident on Mars - layers below the surface are fully formed (full of life), magnetic field receded leaving the surface sterilized. Delivered water froze and is now covered with dust. Thus, one can only expect to find residual and resilient bacteria within the crust of Mars.

Similar happened on Venus except water evaporated due to high surface temperature.

Nothing in nature is linear (although this approximation may be suitable during stages of weak evolution) and in these extreme events one can expect significant departures from linear relationships (in multiple orders of magnitude) between phenomena.

Since these events are coupled with gravitational stresses of the Solar System one can expect temporary but significant increase in alpha and neutrino radiation (radiation flux induced by temporary collapse of a gravitational well - strongly affecting half-lives of isotopes).

Note that all decay types are affected equally since changes are caused by time dilation due to scale change.

Also note that these changes are synchronized with orbital decays in the Solar System - which, like the decay rates, are accelerated during the pulse but return to normal after the pulse.

Due to universal synchronization and restoration of previous equilibrium states it is hard to detect such pulses - in fact, astronomical and geological observations will not reveal any deviation from constancy of decay rates. 
Thus, with such nature of cataclysmic changes, the principle of uniformitarianism will inevitably seem, but cannot be, valid.

Note also that most of the emitted radiation will be lost to space for the same reason - temporary collapse of gravitational/electro-magnetic well, thus solving the problem of missing radiogenic Helium ${ }^{41}$. Due to conservation of momentum, significant loss of heavier atmospheric particles is not expected due to well loss, but can occur during the short exposure to solar wind.

The assumption of constant decay rates will not only produce incorrect ages but can result in misplacement of events on a geological timescale. Thus, inconsistencies in certain geological records can serve as indirect evidence to disruptions in decay rates.

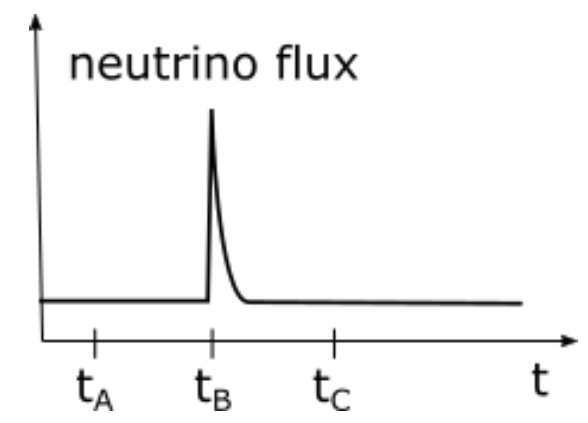

Fig. 6: Neutrino pulse due to decay rate increase

Consider the neutrino pulse on Fig. 6 - under the assumption of constant decay rates, $\mathbf{3}$ different fossil records $\mathbf{A}, \mathbf{B}, \mathbf{C}$ may give following results:

- assuming non-isotropic space-time perturbation, such that fossil record $\mathbf{A}$ decay is not affected by the event at $t_{B}$, the event at $t_{B}$ (associated with fossil record $B$ ) might appear to have happened before the event at $t_{A}$ (associated with fossil record $A$ )

- in case decay rates of both $\mathbf{A}$ and $\mathbf{B}$ are affected, the distance of $t_{A}$ and $t_{B}$ to $t_{C}$ will be increased (time interval expansion)

Neutrino flux can also be decreased resulting in shortening (rather than expansion) of time intervals, although in this context the increase of the flux should prevail.

Due to accumulation, the duration of fossilized events would apparently increase with time so older events would seem longer in duration compared to more recent events. This is exactly the case with current fossil evidence of past carbon cycle disruptions.

The effects are illustrated on Fig. 4 b) - the boundaries of the middle icehouse period might match the (305 \& 252 mya) extinctions if decay rates would be constant. 
In such case, the current rate of $\mathrm{CO}_{2}$ injection is not different from those in previous major extinctions (the fact that it is anthropogenic makes no difference to the universe - it is only humans making humans special).

Previously obtained period of $\mathbf{2 7}$ million years does not take into account time compression - it should be shorter and close to a multiple of Earth's period of existence $\left(T_{x}=1.512 * 10^{6}\right.$ years). In case of ideal synchronization, the real period is:

$$
T_{d}=\left\lfloor\frac{26 * 10^{6}}{1.512 * 10^{6}}\right\rfloor 1.512 * 10^{6}=25.704 * 10^{6} \text { years }
$$

This is in agreement with determined periodicity of impact cratering of $25.8 \pm 0.6 * 10^{6}$ years ${ }^{42}$.

One can now assume that the $\mathbf{C O}_{2}$ injection right before the Cretaceous-Tertiary (KT) boundary $(65$ mya) is equal to current (anthropogenic) injection and that radiation is induced during the injection - with the start of injection corresponding to $t_{A}$ and end of injection to $t_{C}$ on Fig. 6.

Given the $\mathrm{CO}_{2}$ increase from $780 \mathrm{ppmv}$ to $1440 \mathrm{ppmv}\left(\Delta \mathrm{CO}_{2}=660 \mathrm{ppmv}\right)$ in period 66.5 mya -65.5 mya $\left(\Delta \mathrm{t}_{\mathrm{i}}=1 \text { million years }\right)^{43}$, compression of time $\Delta \mathrm{t}_{\mathrm{c}}$ with each extinction:

$$
\Delta t_{c}=\Delta t_{i}-\Delta t_{a i}=1 * 10^{6}-234=999766 \text { years }
$$

where $\Delta t_{a i}$ is the period of 660 ppmv of anthropogenic $\mathrm{CO}_{2}$ increase since year 1850, calculated using (C1.1).

Such compression of time is easily achievable using C1.2. In example, for ${ }^{10} \mathrm{Be}$ :

$$
T_{1 / 2}=2 * 1.512 * 10^{6}-\frac{1.512 * 10^{6}}{341.83707500861} * 300 *\left(\frac{6}{5} * 2^{45 x^{2}}\right)^{x}
$$

Half-life of ${ }^{10} \mathrm{Be}$ decreasing by the above equation, reaches required time compression in year 2065 , on day 66 of the year.

Source code:

getage.php +

Number of 3rd order cycles of existence since Cretaceous-Paleogene extinction event (66 mya):

$$
n=\left\lfloor\frac{t_{K P g}}{T_{x}}\right\rfloor=\left\lfloor\frac{66 * 10^{6}}{1.512 * 10^{6}}\right\rfloor=43
$$


Gravitational collapse during strong evolution pulses (occurring with a period of $\mathbf{T}_{\mathrm{x}}$ years) lasts only $\Delta \mathrm{t}_{\mathrm{n}_{\mathrm{x}}}=$ 19.3 seconds, but the collapse during stronger evolution pulses (occurring with a period of $T_{d}$ years) lasts longer (possibly 7 days).

With each extinction, gravitational collapse of the Sun releases the pressure from condensed energy beyond the surface event horizon and the Sun effectively starts expanding.

The expansion reaches the orbit of Mars before the gravitational well is restored, so, assuming expansion at the speed of light, time of increased decay radiation is:

$$
\Delta t_{n_{d}}=\frac{r_{M}}{c}=\frac{227.92 * 10^{9}}{2.99792458 * 10^{8}}=760.259 \mathrm{~s}=12.671 \mathrm{~m}
$$

where $r_{M}$ is the distance of Mars to Sun.

Now one can calculate time compression with each cycle (pulse) of existence $\Delta t_{C_{x}}$ and each extinction $\Delta \mathrm{t}_{\mathrm{C}_{\mathrm{d}}}$ :

$$
\begin{gathered}
\Delta t_{c}=\Delta t_{c_{d}}+\Delta t_{c_{x}} \\
\frac{\Delta t_{c_{d}}}{\Delta t_{c_{x}}}=\frac{\Delta t_{n_{d}}}{\Delta t_{n_{x}}} \\
\Delta t_{c_{x}}=\Delta t_{c} \frac{1}{\frac{1}{\Delta t_{n_{d}}}+1}=24751.794 \text { years } \\
\Delta t_{c_{d}}=\Delta t_{c}-\Delta t_{c_{x}}=975014.206 \text { years }
\end{gathered}
$$

Age of Earth is thus overestimated by:

$$
\sigma_{T_{E}}=\left\lfloor\frac{\Delta T_{E_{i m g}}}{T_{d}}\right\rfloor \Delta t_{c_{d}}+\left\lfloor\frac{\Delta T_{E_{i m g}}}{T_{x}}\right\rfloor \Delta t_{c_{x}}=245907386 \text { years }
$$

giving the real age of Earth:

$$
\Delta T_{E}=\Delta T_{E_{i m g}}-\sigma_{T_{E}}=4.29409 \pm 0.05 * 10^{9} \text { years }
$$

where $\Delta \mathrm{T}_{\mathrm{E}_{\text {img }}}=4.54 \pm 0.05 * 10^{9}$ years.

If one assumes that $\mathbf{T}_{d}$ is the equivalent of $\mathbf{1}$ day of human embryo development, Earth is at the week 25 
(GW25) of gestation period (right at the beginning, in case of corrected age).

The GW25 marks the end of embryonic neurogenesis in humans and thus agrees with the hypothesis of final major extinction.

The current carbon cycle disruption (6th major extinction) will thus not span thousands $(\sim 10000)$ of years as predicted by the assumption of constant decay, but at most 234 years - starting from year 1850 (10000 years of already passed Holocene extinction may be regarded as a precursor to the major event starting at year 1850).

$$
1850+234=2084
$$

Note that this year corresponds to $950 \mathrm{ppm}$, as predicted by (C1.1).

The gravitational well of the Sun collapses on average every $\mathbf{1 . 5 1 2}$ million years. At that point the Sun is in a state of a white dwarf for $\mathbf{1 9 . 3}$ seconds before the well is restored.

This is a part of a normal ${ }^{10}(\mathrm{C}-\mathrm{B}-\mathrm{Be})$ oscillation cycle of a single atom in a low density environment.

A similar mechanism must be behind the longer lasting collapse happening on average every 25.704 million years. This, however, may be the exchange of gravitational maximums (gluons) between multiple atoms.

The other atom must cycle through states in a similar way - different atoms at each end of the chain, one with short lifetime, other with a long lifetime, and a shared decay product in between, which, at $\mathbf{U}^{0}$ has an extremely long (stable) half-life.

The long lifetime (half-life) at one end of this chain should be $25.704 * 10^{6}$ years.

However, most recent measurement of ${ }^{10} \mathrm{Be}$ half-life yielded a value of $\mathbf{1 . 3 8 8 \pm 0 . 0 1 8}$ myr ${ }^{44}$ so one might assume that the measured half-life of this element too will be lower. The half-life to look for is thus:

$$
T_{1 / 2} \approx \frac{1.388 \pm 0.018}{1.512} * 25.704 * 10^{6}=23.596 \pm 0.018 * 10^{6} y
$$

The half-life closest to this value is the one of ${ }^{236} \mathrm{U}$ (Uranium), which, according to measurement from 1980., has a half-life of $23.42 \pm 0.03 * 10^{6}$ years.

The decay product of ${ }^{236} \mathrm{U}$ is ${ }^{232}$ Th (Thorium) so this isotope is in the middle of the chain.

Assuming horizontal conservation of half-life ratios, a total (short) half-life at the other end of the chain should be: 


$$
T_{1 / 2}=\frac{19.3}{1.512} 25.704=328.1 s
$$

A plausible cycle is thus $\left({ }^{236} \mathrm{U}-?\right)-{ }^{232} \mathrm{Th}-\left({ }^{232} \mathrm{Ac}-{ }^{232} \mathrm{Ra}\right)$.

In any case the mass of this system is $\mathbf{2 3 . 6}$ Solar masses which means it oscillates between an $\mathbf{O}$ class star and an inactive state in the form of a neutron star or a black hole.

The source of such systems is the energetic disc concentrated around a galactic gluon (dark matter ring) in the galactic midplane through which Solar System passes roughly every $\mathbf{3 0}$ million years ${ }^{45}$.

However, a direct interaction between such system and Solar is unlikely. A more plausible interaction is that of a Solar System and an alpha particle emitted during ${ }^{236} \mathbf{U}$ decay.

The alpha particle at $\mathbf{U}^{\mathbf{0}}$ is stable so it's half-life at $\mathbf{U}^{\mathbf{1}}$ is $\approx \mathbf{0}$, therefor it oscillates in a ${ }^{\mathbf{4}}(\mathrm{H}-\mathrm{He}-\mathrm{Li})$ cycle.

Assuming gluon speed equal to c, distance from the Sun to alpha gluon at the point of gluon exchange must be equal to $1 \mathrm{MAU}$ (effective range of the strong force):

$$
c \Delta t_{n_{d}}=2.99792458 * 10^{8} * 760.259=227.92 * 10^{9} \mathrm{~m}
$$

The interaction causes orbital perturbations in the Solar System and may be a plausible source for predicted asteroid and cometary bombardment.

\subsubsection{Sea level changes}

Neurogenesis requires transfer of differentiated progenitor cells to subterranean world, into designated mantle layers. Therefor, a passageway must exist somewhere, connecting the surface with underground tunnels leading to such places - unless one is created when needed, which I find unlikely.

Scaling the largest neuron cells to Earth size, such passageway must have a radius of at least $\approx 250$ meters to allow sequential cell transfer. However, parallel transfer of multiple cells is certainly more plausible - a radius on the order of $10^{4} \mathrm{~m}$.

Thus, the only location where this could remain hidden (protected) and isolated when unused is Antarctica. Ice melting is required to expose this location but also to rise the sea level in order to pick up the cells on land area.

Rise in atmospheric greenhouse gases and consequent rise in temperature is inadequate to melt all ice in the predicted short time-frame. Thus, a different mechanism must be responsible to induce breaking and melting of ice sheets - volcanism and/or asteroid bombardment. 


\subsubsection{Analysis of past extinctions}

Here, past extinctions are analyzed for periodicity, with incorporated corrections by previously calculated time compression due to pulses of decay rate changes.

Periodicity is obtained using circular spectral analysis ${ }^{46}$ of a couple of datasets, which all give similar results.

Data is grouped into energy levels corresponding to the extinction magnitude (5 - major extinctions, 4 minor extinctions, 3 - other extinctions, 2 and 1 - potential extinctions).

\section{The method}

In the circular model of periodicity a time line is wrapped around a circle, the circumference of which represents a trial period. For each occurrence, a unit vector from the origin is calculated. If periodic, the series will tend to form a cluster at one point on the circumference when the correct trial period is used. Here, angular location relative to $0^{\circ}$ (present) gives the phase $\left(\mathbf{t}_{\mathbf{0}}\right)$.

Ages of individual events $\left(\mathbf{t}_{\mathbf{i}}\right)$ are transformed to angles $\left(\mathbf{a}_{\mathbf{i}}, \mathbf{b}_{\mathbf{i}}\right)$ for each trial period $\mathrm{P}$ :

$$
\begin{gathered}
a_{i}=\sin \left(\frac{2 \pi}{P} t_{i}\right) \\
b_{i}=\cos \left(\frac{2 \pi}{P} t_{i}\right) \\
S=\frac{1}{N} \sum_{i=1}^{N} a_{i} \\
C=\frac{1}{N} \sum_{i=1}^{N} b_{i} \\
R=\sqrt{S^{2}+C^{2}}
\end{gathered}
$$

where $\mathbf{R}$ is a mean vector magnitude (normalized measure of goodness of fit). The phase shift (to) is calculated as follows:

$$
\begin{array}{cc}
t_{0}=\frac{P}{2 \pi} \tan ^{-1}\left(\frac{S}{C}\right) \quad(\text { for } C>0) \\
t_{0}=\frac{P}{2}+\frac{P}{2 \pi} \tan ^{-1}\left(\frac{S}{C}\right) \quad(\text { for } C<0)
\end{array}
$$




\section{Dataset 1}

\begin{tabular}{|c|c|c|}
\hline $\begin{array}{l}\text { energy } \\
\text { level }\end{array}$ & extinction events [mya] & extinction events $\left(t_{i}\right)$, age corrected [mya] \\
\hline 5 & $66^{*}, 201.3^{*}, 252.2^{*}, 365,445$ & $61.986,190.208,238.316,345.385,421.148$ \\
\hline 4 & $37.8^{\star}, 145^{\star}, 260^{a}, 305^{b}, 420^{c}$ & $36.206,136.774,245.993,288.3,397.519$ \\
\hline 3 & $\begin{array}{l}11.6^{*}, 93.9^{*}, 182.7^{*}, 230^{d}, 270,424^{e}, 428^{f}, 488^{g} \\
502\end{array}$ & $\begin{array}{l}11.402,88.465,172.88,217.463,255.844,401.469,404.42,461.48 \\
475.257\end{array}$ \\
\hline 2 & $117^{\mathrm{h}}, 168.3^{*}$ & $111.194,159.702$ \\
\hline
\end{tabular}

Table 1: Extinction events dataset 1, sources: ${ }^{47}, a^{48}, b^{49}, c^{50}, d^{51}, e^{52}, f^{53}, g^{54}, h^{55}$

Extinction events in dataset 1, grouped into energy levels and calculated corrected ages for these events, respectively, are shown in Table 1.

Maximal $\mathbf{R}$ was obtained for a period $\mathbf{P}=\mathbf{2 5 . 9 2}$ My (million years), with a phase of $\mathbf{9 . 3 5 5}$ My.
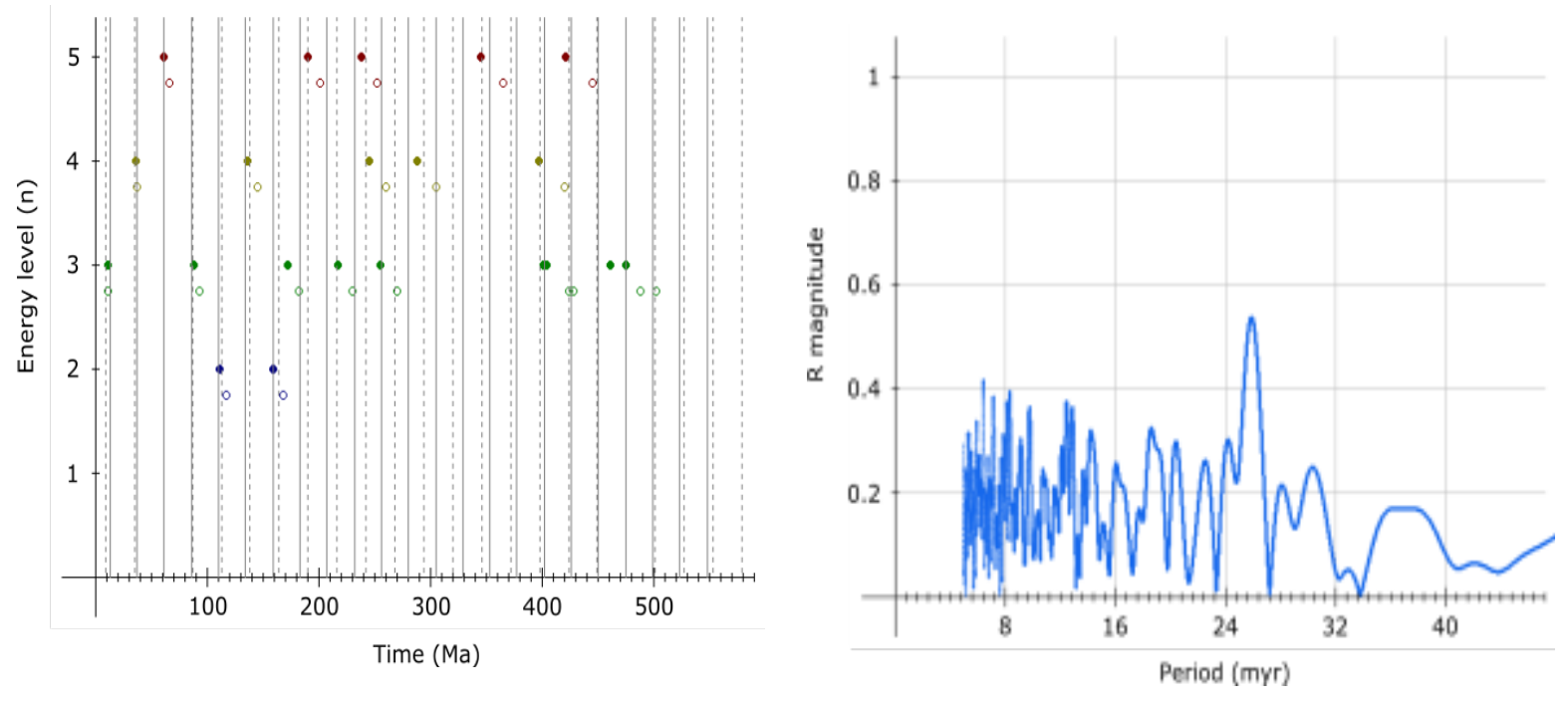

Fig. 1: Extinctions (left), spectral analysis (right)

On the left, Fig. 1 shows extinctions plotted against the obtained periodicity (dashed gray line), solid colored circles are extinction events with corrected ages, empty circles are extinctions with non-corrected ages. On the right, Fig. 1 shows the result of circular spectral analysis.

\section{Dataset 2}

Here, a larger dataset from a single source was used. 
5

4

3

2
$66,201.4,251.9,372.2,445.2$

$37.8,145,259.8,306.7,419.2$

$11.6,93.9,183.7,228.5,272.3,423,427.4,485.4$, 500.5

$113.1,168.3$
$61.986,190.308,238.041,352.461,421.348$

$36.206,136.774,245.793,289.975,396.744$

$11.402,88.465,173.88,215.987,257.12,400.469,403.82,458.929$, 473.782

$107.344,159.702$

Table 2: Extinction events dataset 2, source: Gradstein $2016^{56}$

Maximal $\mathbf{R}$ reveals a period $\mathbf{P}=\mathbf{2 6} \mathrm{My}$, with a phase of $8.617 \mathrm{My}$.
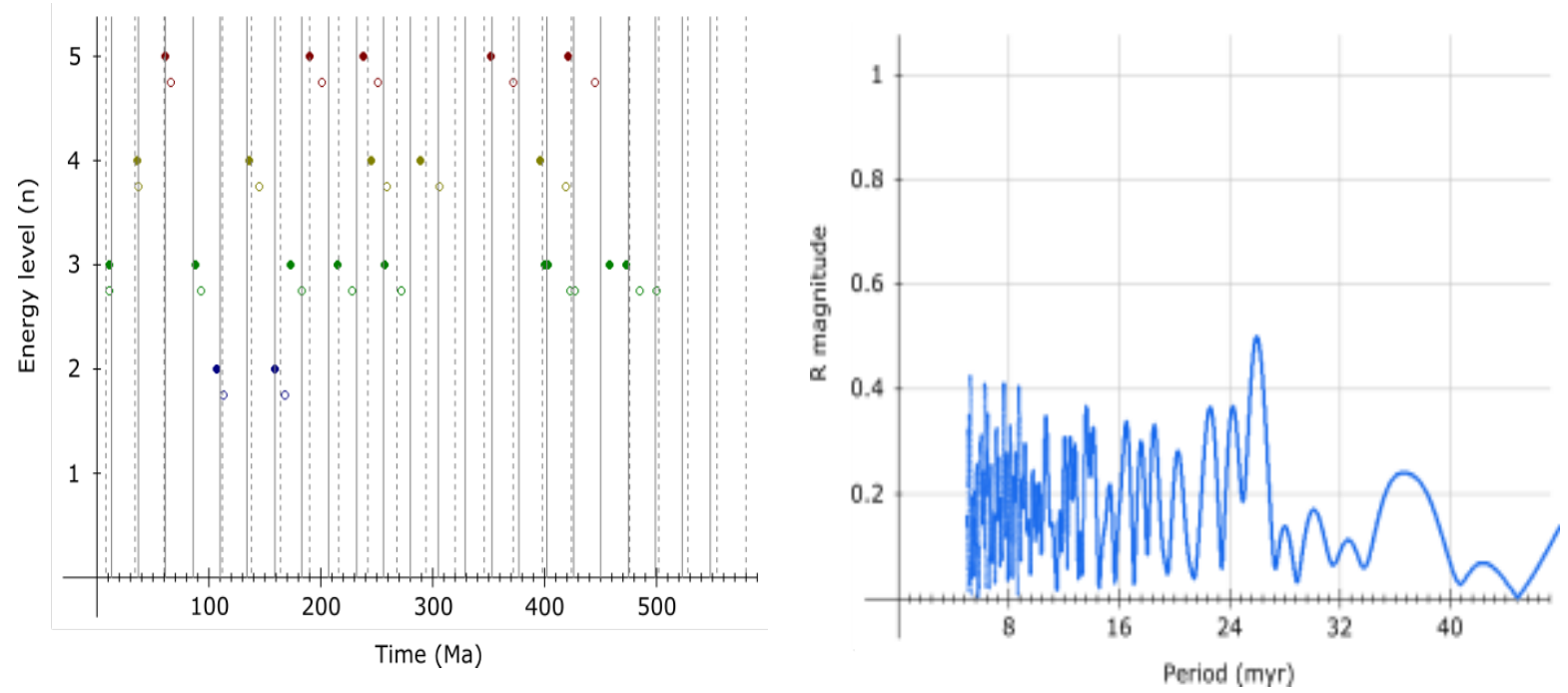

Fig. 2: Extinctions (left), spectral analysis (right)

Extinctions and the result of spectral analysis are shown in Fig. 2.

\section{Dataset 3}

Previous datasets do not take into account possible splitting of energy levels. Here, an even larger dataset is presented which shows possible energy splitting and how this, when not accounted for, causes lower confidence in calculated $\mathbf{P}$.

\begin{tabular}{lll}
$\begin{array}{l}\text { energy } \\
\text { level }\end{array}$ & extinction events [mya] & extinction events $\left(\mathbf{t}_{\mathrm{i}}\right)$, age corrected [mya] \\
\hline 5 & $66,201.4,251.9,372.2,445.2$ & $61.986,190.308,238.041,352.461,421.348$ \\
\hline 4 & $37.8,145,259.8,306.7,419.2,514$ & $36.206,136.774,245.793,289.975,396.744,486.084$ \\
\hline 3 & $11.6,93.9,183.7,228.5,272.3,423,427.4,485.4$, & $11.402,88.465,173.88,215.987,257.12,400.469,403.82,458.929$, \\
\hline 2 & $500.5,541$ & $473.782,511.664$ \\
\hline 1 & $113.1,168.3,330.9$ & $107.344,159.702,312.804$ \\
\hline
\end{tabular}


Table 3: Extinction events dataset 3, source: Gradstein $2016^{57}$

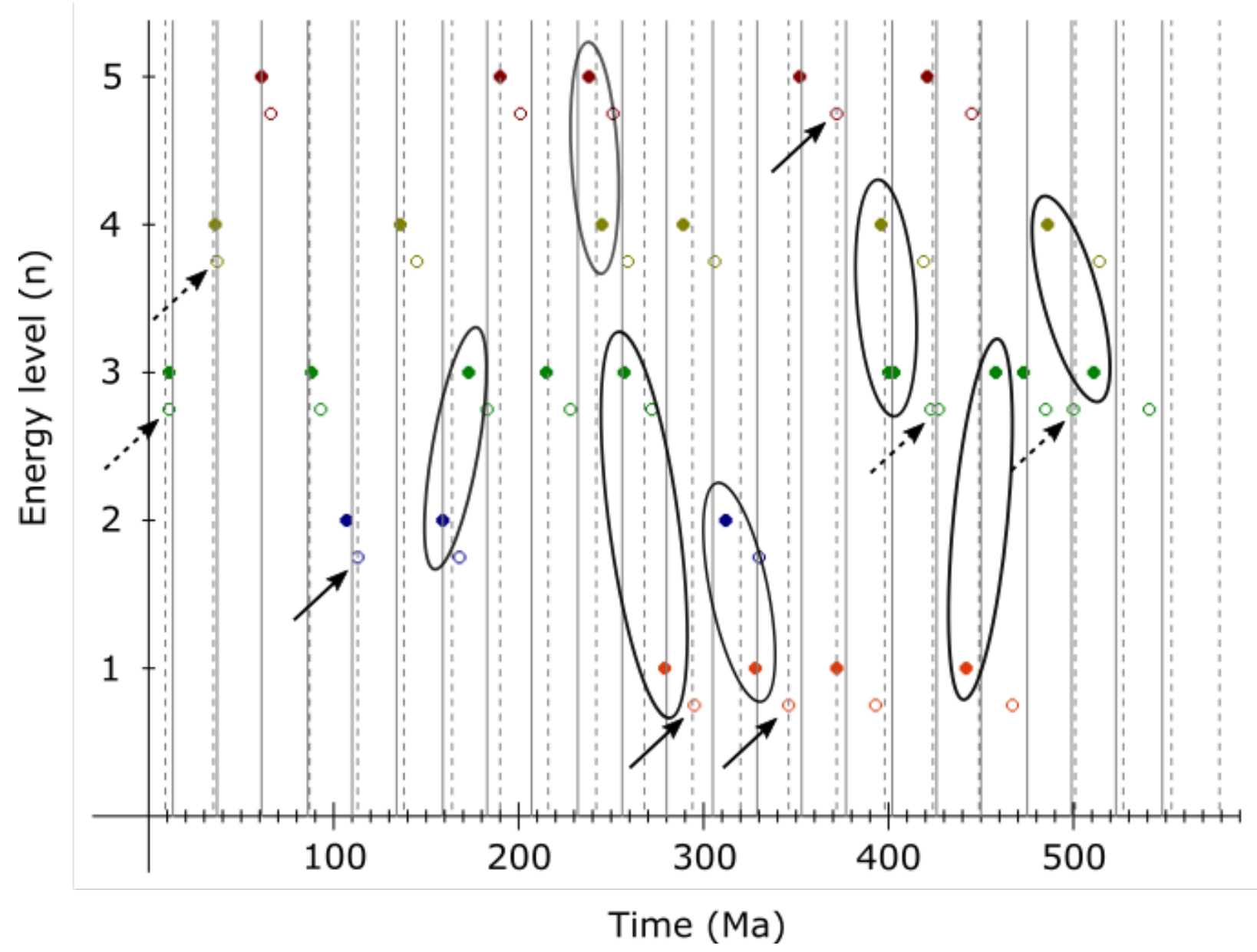

Fig. 3: Extinctions

Here, for $\mathbf{R}=\mathbf{0 . 4 1 3}$, obtained $\mathbf{P}=\mathbf{2 2 . 4 9 3} \mathrm{My}$, phase $\mathbf{1 5 . 6 0 3}$ My.

\section{Dataset 4}

Here I hypothesize that deviations from $\mathbf{P}$ are the result of energy splitting into smaller events which when grouped properly would fit on $\mathbf{P}$ intervals.

The dataset is the same as dataset 3, except the hypothesized splittings (circled extinction pairs on Fig. 3) have been grouped into a single event, simply by using arithmetic mean age of the pair.

\begin{tabular}{|c|c|c|}
\hline $\begin{array}{l}\text { energy } \\
\text { level }\end{array}$ & extinction events [mya] & extinction events $\left(t_{i}\right)$, age corrected [mya] \\
\hline 5 & $66,201.4,(251.9+259.8) / 2=255.9,372.2,445.2$ & $61.986,190.308,241.967,352.461,421.348$ \\
\hline 4 & $37.8,145,306.7,(419.2+423) / 2=421.1,(514+541) / 2=527.5$ & $36.206,136.774,289.975,398.619,499.361$ \\
\hline 3 & $\begin{array}{l}11.6,93.9,(183.7+168.3) / 2=176,228.5,(272.3+295) / 2=283.7,427.4 \\
(485.4+467.3) / 2=476.4,500.5\end{array}$ & $\begin{array}{l}11.402,88.465,166.304,215.987,268.346 \\
403.82,451.053,473.782\end{array}$ \\
\hline
\end{tabular}


2

1
113.1, $(330.9+346.7) / 2=339$

393.3
$107.344,320.78$

372.239

Table 4: Extinction events dataset 4
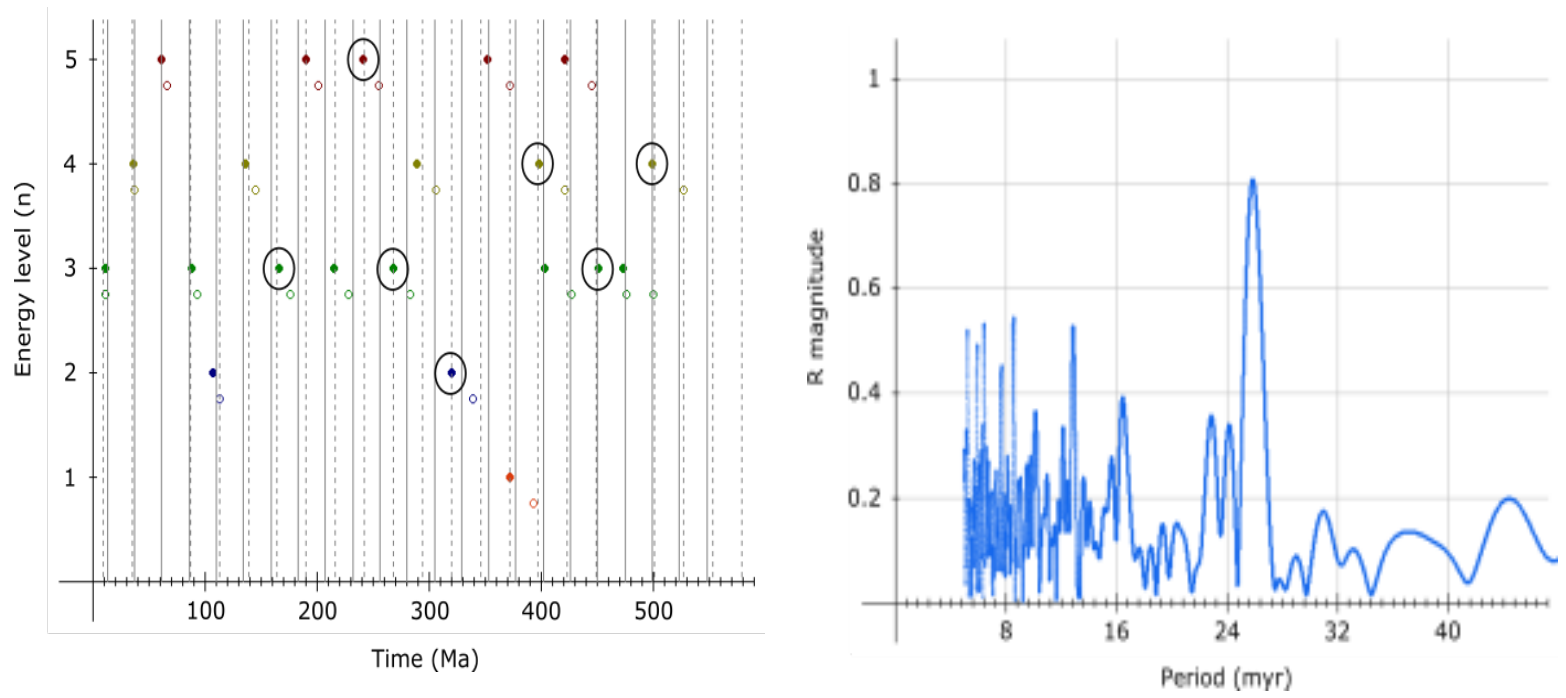

Fig. 4: Extinctions (left), spectral analysis (right)

The $\mathbf{R}$ peaks at $\mathbf{0 . 8 0 7}$, corresponding to $\mathbf{P}=\mathbf{2 5 . 8 9} \mathrm{My}$, very close to one obtained from dataset $\mathbf{1}$. Phase is $9.55 \mathrm{My}$.

\section{Dataset 5}

Here dataset 4 is modified with the assumption that splitting occurs in all events, thus, in addition to previously grouped events, the remaining non-grouped events have been grouped with adjacent boundaries.

\begin{tabular}{|c|c|c|}
\hline $\begin{array}{l}\text { energy } \\
\text { level }\end{array}$ & extinction events [mya] & $\begin{array}{l}\text { extinction events }\left(t_{i}\right) \text {, age } \\
\text { corrected [mya] }\end{array}$ \\
\hline 5 & $\begin{array}{l}(61.6+66) / 2=63.8,(199.4+201.4) / 2=200.4,(251.9+259.8) / 2=255.9,(372.2+382.7) / 2= \\
377.5,(443.8+445.2) / 2=444.5\end{array}$ & $\begin{array}{l}60.81,189.333,241.967,356.687 \\
420.648\end{array}$ \\
\hline 4 & $\begin{array}{l}(33.9+38) / 2=36,(139.4+145) / 2=142.2,(306.7+314.6) / 2=310.7,(419.2+423) / 2=421.1 \\
(514+541) / 2=527.5\end{array}$ & $\begin{array}{l}34.431,134.998,293.926,398.619 \\
499.361\end{array}$ \\
\hline 3 & $\begin{array}{l}(11.6+13.8) / 2=12.7,(89.8+93.9) / 2=91.9,(183.7+168.3) / 2=176,(228.5+237) / 2=232.8 \\
(272.3+295) / 2=283.7,(427.4+430.5) / 2=429,(485.4+467.3) / 2=476.4,(497+500.5) / 2= \\
498.8\end{array}$ & $\begin{array}{l}12.502,86.49,166.304,220.213 \\
268.346,405.395,451.053,472.107\end{array}$ \\
\hline 2 & $(113.1+126.3) / 2=119.7,(330.9+346.7) / 2=339$ & $112.87,320.58$ \\
\hline 1 & $(387.7+393.3) / 2=390.5$ & 369.489 \\
\hline
\end{tabular}

Table 5: Extinction events dataset 5 


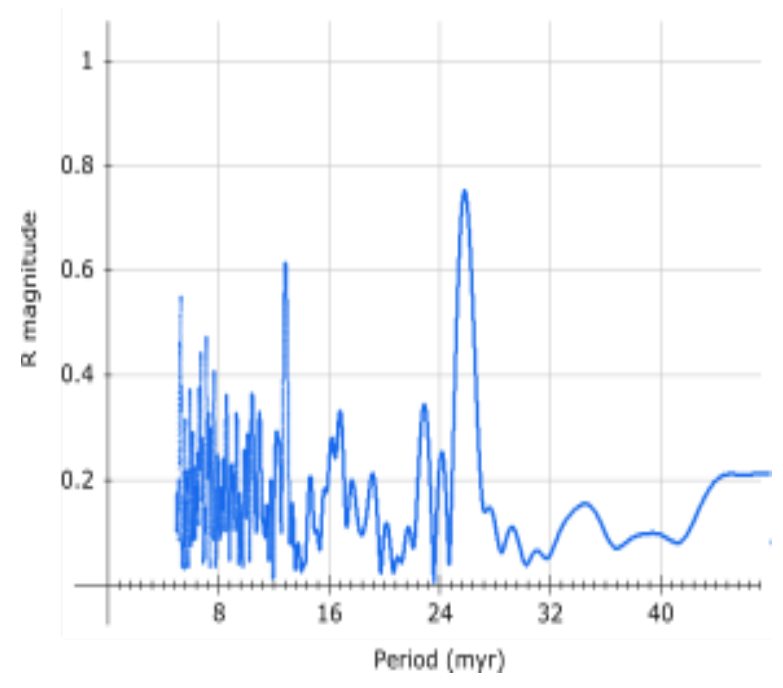

Fig. 5: Dataset 5, spectral analysis

The $\mathbf{R}$ peaks at $\mathbf{0 . 7 5}$, corresponding to $\mathbf{P}=\mathbf{2 5 . 8 4} \mathrm{My}$. Phase for this $\mathbf{P}$ is $\mathbf{9 . 7 8} \mathrm{My}$, however, here another peak at $12.875 \mathrm{My}(\mathrm{R}=\mathbf{0 . 6 1})$ reveals a likely harmonic.

\section{Dataset 6}

Here, dataset contains only highest energy (major and minor) extinctions, from dataset 1.

\begin{tabular}{lll} 
energy level & extinction events [mya] & extinction events $\left(\mathbf{t}_{\mathrm{i}}\right)$, age corrected [mya] \\
\hline $\mathbf{5}$ & $\mathbf{6 6}, \mathbf{2 0 1 . 3}, \mathbf{2 5 2 . 2}, \mathbf{3 6 5 , 4 4 5}$ & $\mathbf{6 1 . 9 8 6}, \mathbf{1 9 0 . 2 0 8 , 2 3 8 . 3 1 6 , 3 4 5 . 3 8 5 , 4 2 1 . 1 4 8}$ \\
\hline 4 & $37.8,145,260,305,420$ & $36.206,136.774,245.993,288.3,397.519$
\end{tabular}

Table 6: Extinction events dataset 6

This dataset gives highest $\mathbf{R}$ maximum (0.837), a period $\mathbf{P}=\mathbf{2 5 . 7 4} \mathrm{My}$, with a phase of $\mathbf{9 . 6 8 9} \mathrm{My}$.

\section{Confidence}

Note that equal weight was assumed for all extinctions in a particular dataset. Different weights can affect the confidence in the result (less if they are all harmonics). But even with that taken into account, there is high confidence in $P \approx 25.74$ My - 25.89 My.

The result with highest confidence $(25.74 \mathrm{My})$ is also the closest to calculated ideal quantization by the 3rd order period $\left(1.512 * 10^{6} \mathrm{My}\right)-\mathbf{2 5 . 7 0 4} \mathrm{My}$, further increasing confidence in such periodicity. 
Note that the burning cycle of the Sun's core is calculated (in the "Quantization of the Sun" chapter) to be equal to $25.746608 \mathrm{My}$, confirming the signal.

Neurogenesis in lifeforms on Earth during embryonic development does imply certain periodicity in the formation of brain layers and neuron migration.

High energy impact cratering and extinctions (migrations) in planetary neurogenesis should be no exception.

In fact, with such periodicity and the last high energy extinction 37.8 My in the past, next one is overdue, roughly by the phase shift.

Note that such delay of extinction may have some benefits due to more evolved precursor neurons at time of differentiation, although with the cost of increased probability of cancer development.

Thus, imminent major extinction as calculated using models based on C1.1 equation should not be surprising.

\section{Correlation with mantle layers}

Grouping and correlation of extinction events with the formation of brain [mantle] layers also indicates that another major mass extinction should be near, at least in geological terms.
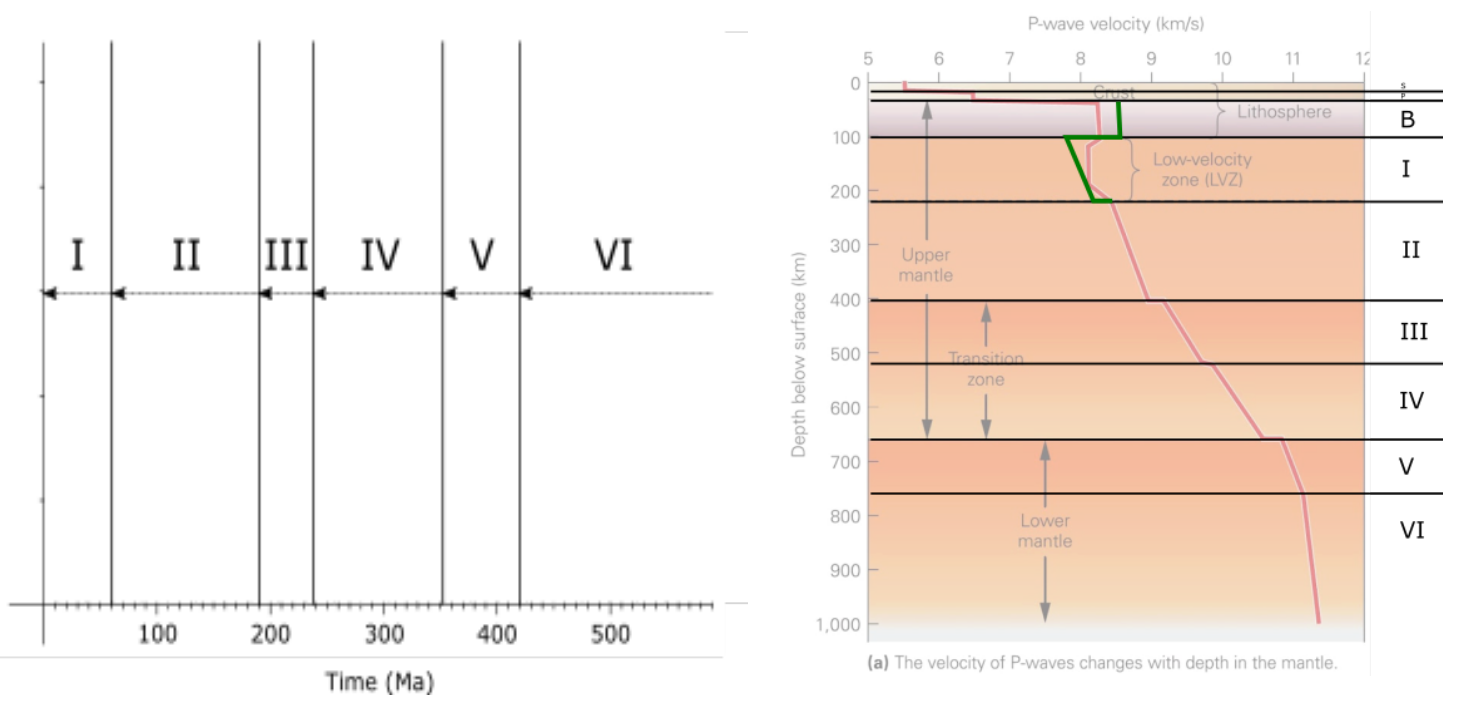

Fig. 5: Correlation of major extinctions (left) with Earth's mantle layers (right)

This correlation is shown on Fig. 5 - time between major extinction events of Phanerozoic is roughly proportional to thickness of a corresponding mantle layer. 
Such correlation should not be surprising - all lifeforms grow in layers.

This is, effectively, a conversion of time separated discontinuities into events separated in space.

To quantify the correlation, periods of weak evolution of mantle layers and thicknesses have been normalized:

$$
\begin{aligned}
& T_{n}(i)=\frac{T(i)}{\sum_{j=1}^{N} T_{j}} \\
& D_{n}(i)=\frac{D(i)}{\sum_{j=1}^{N} D_{j}}
\end{aligned}
$$

Results are shown in Table 6.

\begin{tabular}{|l|l|l|l|l|}
\hline $\mathrm{i}$ & $\begin{array}{l}\text { Period of weak evolution T } \\
{[\mathrm{My}]}\end{array}$ & $\begin{array}{l}\text { Normalized period of } \\
\text { weak evolution } \mathrm{T}_{\mathrm{n}}\end{array}$ & $\begin{array}{l}\text { Corresponding mantle layer } \\
\text { thickness D }[\mathrm{km}]\end{array}$ & $\begin{array}{l}\text { Normalized layer } \\
\text { thickness } \mathrm{D}_{\mathrm{n}}\end{array}$ \\
\hline $\mathbf{5}$ & $\mathbf{4 2 1 . 3 4 8 - 3 5 2 . 4 6 1 = 6 8 . 8 8 7}$ & 0.163 & $780^{\mathrm{b}}-660^{\mathrm{b}}=120$ & 0.176 \\
\hline 4 & $352.461-238.041=114.42$ & 0.272 & $660^{\mathrm{b}}-520^{\mathrm{b}}=140$ & 0.206 \\
\hline 3 & $238.041-190.308=47.733$ & 0.113 & $520^{\mathrm{b}}-410^{\mathrm{b}}=110$ & 0.162 \\
\hline 2 & $190.308-61.986=128.322$ & 0.305 & $410^{\mathrm{b}}-220^{\mathrm{a}}=190$ & 0.279 \\
\hline 1 & $61.986-0=61.986$ & 0.147 & $220^{\mathrm{a}}-100^{\mathrm{b}}=120$ & 0.176 \\
\hline
\end{tabular}

Table 6: Comparison of weak evolution periods and mantle layers, sources: $a^{58}, b^{59}$

Correlation in absolute value varies between the pairs, but in general, taking into account oscillation, the correlation is very good.

At least some discrepancies could be explained by the fact that formation is not yet complete - ie. the boundary between layer 1 and 2 is most likely to change.

If layer 3 decrease would be equal to layer 4 increase $(\approx 0.0575$ in normalized value) and layer 1 decrease to layer 2 increase $(\approx 0.0275 \approx 0.0575 / 2)$, with a small decrease in layer $5(0.013 \approx 0.0275 / 2)$ coupled with equivalent increase in layer $\mathbf{6}$, normalized extinction and mantle boundaries would be almost equal. 
This suggests that extinction events are memorized, all layers change with every extinction but toward a specific predetermined pattern - exactly as expected with DNA encoded evolution of living organisms.

Note that the exact boundaries are a matter of debate. They have some thickness (perhaps due to layer not being fully formed), so it may be more appropriate to equate layer thickness with distance between discontinuities. If that would be a distance between lower discontinuities of two boundaries, it would, at least for layer 1, yield a normalized value exactly equal to the corresponding normalized period of weak evolution (220 $120=100 / 680=0.147)$.

Correlation of layer 6 and the corresponding period of weak evolution has not been determined due to unknown boundary.

However, assuming that extinction at the start of Phanerozoic (511.664 mya in corrected age, or 541 mya non-corrected) is correlated with the lower boundary of layer $\mathbf{6}$, one can calculate the thickness of layer $\mathbf{6}$ :

$$
\begin{gathered}
\frac{T_{6}}{T_{5}}=\frac{D_{6}}{D_{5}} \\
D_{6}=\frac{T_{6}}{T_{5}} D_{5}=\frac{511.664-421.348}{421.348-352.461} 120=157 \mathrm{~km}
\end{gathered}
$$

In that case, a discontinuity, if formed, should exist in Earth's mantle at a depth of $937 \mathrm{~km}$ (assuming boundary between layer 5 and 6 at $780 \mathrm{~km}$ ).

\section{Supplement}

Here is the code used to calculate correct ages of extinction events, perform the analysis and generate images. getext.php + 


\section{Quantization of Moon orbits}

If the gravity of Earth's [major] gravitational maximum is, as hypothesized, equal to surface gravity of the Sun, one would expect for orbitals of natural moons of Earth to be scaled orbitals of inner planets.

Allowed orbitals are thus:

$$
r=\frac{r_{p}}{R_{\odot}} r_{c}
$$

where $\mathbf{r}_{\mathbf{c}}$ is the Earth's gravitational maximum radius (= inner core radius), $\mathbf{R}_{\odot}$ is the radius of the Sun and $\mathbf{r}_{\mathbf{p}}$ is the orbital radius of a corresponding planet.

Using $R_{\odot}=695735 \mathrm{~km}, r_{c}=1206.115 \mathrm{~km}$, one obtains following orbitals:

\begin{tabular}{|c|l|l|}
\hline entanglement & $r_{p}(\mathbf{k m})$ & $r(\mathbf{k m})$ \\
\hline Mercury & 57910000 & 100392 \\
\hline Venus & 108210000 & 187591 \\
\hline Earth & 149600000 & 259344 \\
\hline Mars & 227920000 & 395118 \\
\hline
\end{tabular}

Evidently, the Moon is currently at the scaled Mars' orbit. Even the distance between perihelion and aphelion is scaled by equal orders of magnitude - for Mars it is $42.61 * 10^{6} \mathrm{~km}$, while for the Moon, the distance is $42.2 * 10^{3} \mathrm{~km}$.

Small discrepancies should be attributed to oscillation and phase shift in synchronization.

Note that Earth is likely receding from the Sun at the scaled rate of Moon's recession from Earth. 


\section{Quantization of the Sun}

During inflation of the Sun, multiple gravitational maximums were inflating within. Collapse of these maximums as the Sun was deflating was fossilized in the Sun, in the form of discontinuities. As these maximums are now gravitational maximums of inner planets, entanglement exists between radii of discontinuities and planetary orbits.

Some discontinuities are strong (permanent) while some are weak, evolve over time and may periodically disappear. Apparent discontinuities are those between the core, radiative and convective zone, surface discontinuity and the boundaries of tachocline.

Regardless of the configuration $\left(1 \mathrm{e}^{+}\right.$or $\left.2 \mathrm{e}^{+}\right)$, each inner planet formed with the collapse of two neutral spin anti-aligned maximums. Thus, each is entangled with 2 discontinuities in the Sun.

Initial inflation of planetary maximums must have been faster than light to preserve invariance.

If one assumes that all maximums initially had the mass of the Sun and energy density remained constant during inflation, with the collapse (energy level change) occurring once escape velocity was equal to the speed of light (in $\mathbf{C R}$, discontinuities between energy levels are speed limits), orbital radii of planets become fossils of Schwarzschild radii:

$$
\begin{aligned}
r=\frac{2 G m}{c^{2}} & =\frac{2 G \rho V}{c^{2}}=\frac{r^{3}}{R^{3}} \frac{2 G M}{c^{2}} \\
r & =\sqrt{R^{3} \frac{c^{2}}{2 G M}}
\end{aligned}
$$

$$
\begin{array}{r}
\mathrm{R}=\text { initial radius } \\
\mathrm{M}=1.988500 * 10^{30} \mathrm{~kg} \\
\mathrm{C}=\text { standard speed of light }=2.99792458 * 10^{8} \mathrm{~m} / \mathrm{s} \\
\mathrm{G}=6.674 * 10^{-11} \mathrm{~m}^{3} / \mathrm{kgs}^{2}
\end{array}
$$

With equal escape velocity (pressure per surface quantum) between maximums (note that a smaller maximum is inside the other), radius of fusion of two maximums becomes the arithmetic mean of two radii:

$$
r=\frac{1}{2}\left(\sqrt{R_{1}^{3} \frac{c^{2}}{2 G M}}+\sqrt{R_{2}{ }^{3} \frac{c^{2}}{2 G M}}\right)
$$

In that case, discontinuities entangled with planetary orbits are at $\mathbf{1 / 5} \mathbf{R}_{\odot}, \mathbf{2} / \mathbf{5} \mathbf{R}_{\odot}, \mathbf{1 / 2} \mathbf{R}_{\odot}, \mathbf{2} / \mathbf{3} \mathbf{R}_{\odot}$ and $\mathbf{1}$ $\mathbf{R}_{\odot}$. 


\begin{tabular}{|c|c|c|c|c|c|}
\hline Planet & $\mathbf{R}_{\mathbf{1}}$ & $\mathbf{R}_{2}$ & Schwarzschild radius $r\left(10^{6} \mathrm{~km}\right)$ & current orbital radius $\left(1^{6} \mathrm{~km}\right)$ & orbital radius (MAU) \\
\hline Mars & $\mathrm{R} \odot$ & $1 / 2 \mathrm{R} \odot$ & 228.52 & 227.92 & 1 \\
\hline Earth & 2/3 R $\odot$ & $1 / 2 \mathrm{R} \odot$ & 151.59 & 149.6 & $2 / 3$ \\
\hline Venus & 2/3 R $\odot$ & 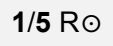 & 107.00 & 108.21 & $1 / 2$ \\
\hline Mercury & 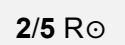 & 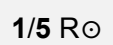 & 57.81 & 57.91 & $1 / 4$ \\
\hline
\end{tabular}

Fig. 1: Correlation of orbital and Schwarzschild radii

Correlation of orbital and Schwarzschild radii is shown on Fig. $\mathbf{1}$, where $\mathbf{R}_{\odot}$ is the radius of the Sun $(695700 \mathrm{~km})$.

Significant orbital eccentricity of Mercury and Mars also seems correlated with Sun's discontinuities.

If Sun's core radius oscillates between $\mathbf{0 . 1}+\mathbf{0 . 1 8 6} \mathbf{R}_{\odot}=\mathbf{0 . 2 8 6} \mathbf{R}_{\odot}$ (hypothesized initial radius) and 1/5 $\mathbf{R}_{\odot}$ (current radius), with constant energy density between the two radii, time independent core radius [as superposition of two oscillatory states] is at $1 / 4 R_{\odot}$.

This is correlated with Mercury's orbit, as its distance from the Sun is at $1 / 4 \mathrm{MAU}$, while its perihelion is at 1/5 MAU.

According to equation $\mathbf{S 1 . 1}$ describing rotational velocities of plasma, and the actual velocity curve, significant points are at $0.1 \mathbf{R}_{\odot}, \approx 1 / 2 \mathbf{R}_{\odot}, 1+0.18686 \mathbf{R}_{\odot}=1.18686 \mathbf{R}_{\odot}$ and $32.8 \mathbf{R}_{\odot}(0.1 \mathrm{MAU}$, half of Mercury's perihelion).

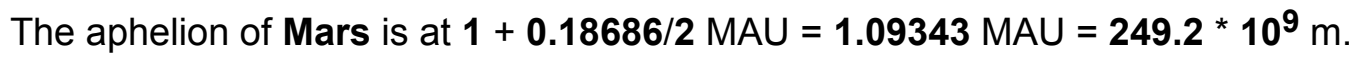

Note that the aphelion of Mars can also be obtained as volumetric mean of Schwarzschild radii associated with 3 discontinuities:

$$
\begin{gathered}
r^{3}=\frac{1}{3}\left\{\left[\left(1 R_{\odot}\right)^{3} \frac{c^{2}}{2 G M}\right]^{\frac{3}{2}}+\left[\left(\frac{2}{3} R_{\odot}\right)^{3} \frac{c^{2}}{2 G M}\right]^{\frac{3}{2}}+\left[\left(\frac{1}{2} R_{\odot}\right)^{3} \frac{c^{2}}{2 G M}\right]^{\frac{3}{2}}\right\} \\
r=249.2 * 10^{9} \mathrm{~m}
\end{gathered}
$$

Similarly, approximate aphelions can be obtained for other planets, ie. for Mercury:

$$
r^{3}=\frac{1}{2}\left\{\left[\left(\frac{2}{5} R_{\odot}\right)^{3} \frac{c^{2}}{2 G M}\right]^{\frac{3}{2}}+\left[\left(\frac{1}{4} R_{\odot}\right)^{3} \frac{c^{2}}{2 G M}\right]^{\frac{3}{2}}\right\}
$$




$$
r=70.4 * 10^{9} \mathrm{~m}
$$

\subsection{Layers of the Sun}

Internal gravity of the Sun depends on the location of maximums and acquired real mass.

Distribution of mass, however, should not be complex unless there are collapsed large scale maximums inside. In any case, matter accumulated between two maximums should, in equilibrium, imitate a maximum and can thus be approximated as one (induced maximum).

One way to obtain gravity of a primordial Sun is to derive it from rotation of real mass - assuming greater rotation with greater gravitational mass, down to the inner core radius $\mathbf{r}_{\mathbf{c}}$, quantization is 1-dimensional (negative):

$$
\frac{1}{g} v r=n h_{2}
$$

Giving the scaled $\mathbf{h}$ constant:

$$
\begin{gathered}
h=h_{2}=5 * 10^{9} \mathrm{~ms} \\
n=1
\end{gathered}
$$

Another way is to assume a completely naked Sun, in which case gravity from the surface down to the core is:

$$
g=g_{p}=G M_{\odot} \frac{r^{2}}{R_{\odot}^{4}}=274 \frac{r^{2}}{R_{\odot}^{2}}
$$

Gravitational profile of the primordial Sun (not taking into account the gravity of inner core maximum) is given in the table below. Here matter velocity $(\mathbf{v})$ is extrapolated from measurements, while space velocity $\left(\mathbf{v}_{\mathbf{s}}\right)$ is calculated from gravity:

$$
\begin{gathered}
v_{s}=\sqrt{g r} \\
v_{p}=\sqrt{g_{p} r}
\end{gathered}
$$

n $\quad$ r/R note

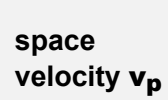

space

velocity $\mathbf{v}_{\mathbf{s}}$

\section{matter} velocity $\mathbf{v}$

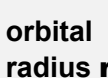

radius $r$ calculated gravity $g_{p}$ $\left(\mathrm{m} / \mathrm{s}^{2}\right)$ calculated gravity $\mathbf{g}$ $\left(\mathrm{m} / \mathrm{s}^{2}\right)$ gravity $\mathbf{g}_{\mathbf{i}}$ ( $v_{c} r$ product) $\mathrm{m} / \mathrm{s}^{2}$ 


\begin{tabular}{|c|c|c|c|c|c|c|c|c|c|}
\hline 1 & 1 & $\begin{array}{l}\text { Convective } \\
\text { discontinuity }\end{array}$ & $\begin{array}{l}436.602565 \\
\mathrm{~km} / \mathrm{s}\end{array}$ & $\begin{array}{l}436.602565 \\
\mathrm{~km} / \mathrm{s}\end{array}$ & $\begin{array}{l}1969.239615 \\
\mathrm{~m} / \mathrm{s}\end{array}$ & $\begin{array}{l}695700000 \\
\mathrm{~m}\end{array}$ & 274 & 274 & $200\left(1^{*} 10^{12}\right)$ \\
\hline 1 & $3 / 4$ & $\begin{array}{l}4 p 6 n \\
\text { discontinuity }\end{array}$ & $\begin{array}{l}283.581685 \\
\mathrm{~km} / \mathrm{s}\end{array}$ & $\begin{array}{l}286.551447 \\
\mathrm{~km} / \mathrm{s}\end{array}$ & $\begin{array}{l}1508.068146 \\
\mathrm{~m} / \mathrm{s}\end{array}$ & $\begin{array}{l}521775000 \\
m\end{array}$ & 154.125 & 157.37 & $\begin{array}{l}150(0.75 \\
\left.* 10^{12}\right)\end{array}$ \\
\hline 1 & $2 / 3$ & $\begin{array}{l}\text { Radiative } \\
\text { discontinuity }\end{array}$ & $\begin{array}{l}234.100417 \\
\mathrm{~km} / \mathrm{s}\end{array}$ & $\begin{array}{l}230.556106 \\
\mathrm{~km} / \mathrm{s}\end{array}$ & $1248 \mathrm{~m} / \mathrm{s}$ & $\begin{array}{l}459162000 \\
m\end{array}$ & 119.3544 & 114.61 & $\begin{array}{l}132(0.66 \text { * } \\
\left.10^{12}\right)\end{array}$ \\
\hline 1 & $1 / 2$ & $\begin{array}{l}\text { 4p6n } \\
\text { discontinuity }\end{array}$ & $\begin{array}{l}154.362317 \\
\mathrm{~km} / \mathrm{s}\end{array}$ & $\begin{array}{l}151.266563 \\
\mathrm{~km} / \mathrm{s}\end{array}$ & $\begin{array}{l}945.454545 \\
\mathrm{~m} / \mathrm{s}\end{array}$ & $\begin{array}{l}347850000 \\
\mathrm{~m}\end{array}$ & 68.5 & 65.78 & $\begin{array}{l}100\left(0.5^{*}\right. \\
\left.10^{12}\right)\end{array}$ \\
\hline 1 & $2 / 5$ & weak & $\begin{array}{l}110.452683 \\
\mathrm{~km} / \mathrm{s}\end{array}$ & $\begin{array}{l}108.233652 \\
\mathrm{~km} / \mathrm{s}\end{array}$ & $\begin{array}{l}756.363636 \\
\mathrm{~m} / \mathrm{s}\end{array}$ & $\begin{array}{l}278280000 \\
m\end{array}$ & 43.84 & 42.1 & $\begin{array}{l}80\left(0.4^{*}\right. \\
\left.10^{12}\right)\end{array}$ \\
\hline 1 & $1 / 4$ & $\begin{array}{l}\text { Outer core } \\
\text { discontinuity }\end{array}$ & $\begin{array}{l}\mathbf{5 4 . 5 7 5 3 2 1} \\
\mathrm{km} / \mathrm{s}\end{array}$ & $\begin{array}{l}91.901023 \\
\mathrm{~km} / \mathrm{s}\end{array}$ & $1396 \mathrm{~m} / \mathrm{s}$ & $\begin{array}{l}173925000 \\
\mathrm{~m}\end{array}$ & 17.125 & 48.56 & $\begin{array}{l}50(0.25 \text { * } \\
\left.10^{12}\right)\end{array}$ \\
\hline 1 & $1 / 5$ & $\begin{array}{l}\text { Inner core } \\
\text { discontinuity = } \\
r_{C}\end{array}$ & $\begin{array}{l}39.050921 \\
\mathrm{~km} / \mathrm{s}\end{array}$ & $\begin{array}{l}74.602949 \\
\mathrm{~km} / \mathrm{s}\end{array}$ & $\begin{array}{l}1437.401179 \\
\mathrm{~m} / \mathrm{s}\end{array}$ & $\begin{array}{l}139140000 \\
\mathrm{~m}\end{array}$ & 10.96 & 40 & $\begin{array}{l}40(0.2 \text { * } \\
\left.10^{12}\right)\end{array}$ \\
\hline
\end{tabular}

Note that multiplying any discontinuity radius with inner core velocity $\mathbf{v}_{\mathbf{c}}$ gives values proportional to $\mathbf{r} / \mathbf{R}$ ratio and gives integer gravity $\left(\mathbf{g}_{\mathbf{i}}\right)$ for inner core and all layers above.

I have previously hypothesized that the Sun has inflated to a much larger radius before being compressed to current one. In the exchange of components of angular momentum, radius might have been exchanged for space (Keplerian) velocity, as shown in table below:

\begin{tabular}{llll} 
discontinuity $(\mathbf{r} / \mathbf{R})$ & space velocity $\mathbf{v}_{\mathbf{S}}$ & correlated radius $\left(\mathbf{1 0}^{\mathbf{6}} \mathbf{\mathrm { km }}\right)$ & possible body correlation \\
\hline 1 & $436.6 \mathrm{~km} / \mathrm{s}$ & 436.6 & end of the main asteroid belt \\
\hline $3 / 4$ & $286.6 \mathrm{~km} / \mathrm{s}$ & 286.6 & beginning of the main asteroid belt \\
\hline $2 / 3$ & $230.6 \mathrm{~km} / \mathrm{s}$ & 230.6 & orbit of Mars (semi-major) \\
\hline $1 / 2$ & $151.3 \mathrm{~km} / \mathrm{s}$ & 151.3 & orbit of Earth (semi-major, aphelion) \\
\hline $2 / 5$ & $108.2 \mathrm{~km} / \mathrm{s}$ & 108.2 & orbit of Venus (semi-major) \\
\hline $1 / 5$ & $74.6 \mathrm{~km} / \mathrm{s}$ & 74.6 & orbit of Mercury (aphelion?)
\end{tabular}

However, orbits may be correlated with arithmetic mean of $\mathbf{v}_{\mathbf{s}}$ and $\mathbf{v}_{\mathbf{p}}$. This gives much better results for the orbit of Mercury - $56.8 * 10^{6} \mathrm{~km}$, agreeing with semi-major, rather than aphelion. Another possibility is entanglement with $\mathbf{v}_{\mathbf{p}}$ instead of $\mathbf{v}_{\mathbf{s}}$. In that case $\mathbf{1 / 4} \mathbf{R}$ discontinuity roughly agrees with the orbit of Mercury.

Remarkable correlations are found subtracting velocities between layers:

discontinuity $(r / R) \quad$ space velocity $v_{s}(\mathrm{~km} / \mathrm{s}) \quad$ correlated radius $\left(1^{6} \mathrm{~km}\right) \quad$ possible body correlation 


\begin{tabular}{|c|c|c|c|}
\hline $1-2 / 3$ & $436.6-230.6$ & 206 & orbit of Mars (perihelion) \\
\hline $2 / 3-1 / 5$ & $230.6-74.6$ & 156 & orbit of Earth (aphelion) \\
\hline $1 / 2-2 / 5$ & $154.4-108.2$ & 46.2 & orbit of Mercury (perihelion)* \\
\hline
\end{tabular}

Difference between current surface gravity and $\mathbf{g}_{\mathbf{i}}$ is roughly equal to the sum of surface gravities of inner and outer planets:

$$
g-g_{i}=274-200=74 \frac{m}{s^{2}}
$$

thus, some entanglement might exist there too.

Below the gravitational minimum at inner core $\left(\mathbf{r}_{\mathbf{c}}\right)$, quantization is 3-dimensional (positive) and gravity should be increasing until the next maximum:

$$
\begin{gathered}
g=n^{2} T \frac{\hbar_{1}}{r^{2}} \\
\hbar_{1}=1.273239545 * 10^{12} \frac{\mathrm{m}^{3}}{\mathrm{~s}^{3}}
\end{gathered}
$$

\subsubsection{Current G model}

Unlike in space above the outer maximum, where gravity falls to zero effectively at infinity (due to next maximum being extremely far), below the maximum gravity falls to zero at finite distance due to compression of space.

With no inner maximums, the single point of zero gravity would be at the center, however, due to relativity, inner maximums must exist (each inner maximum must also be a relative outer maximum).

If the radius of the outer maximum of the Sun is the surface radius, gravity should thus be decreasing below the surface to the point where it is canceled by the [next] inner maximum. 


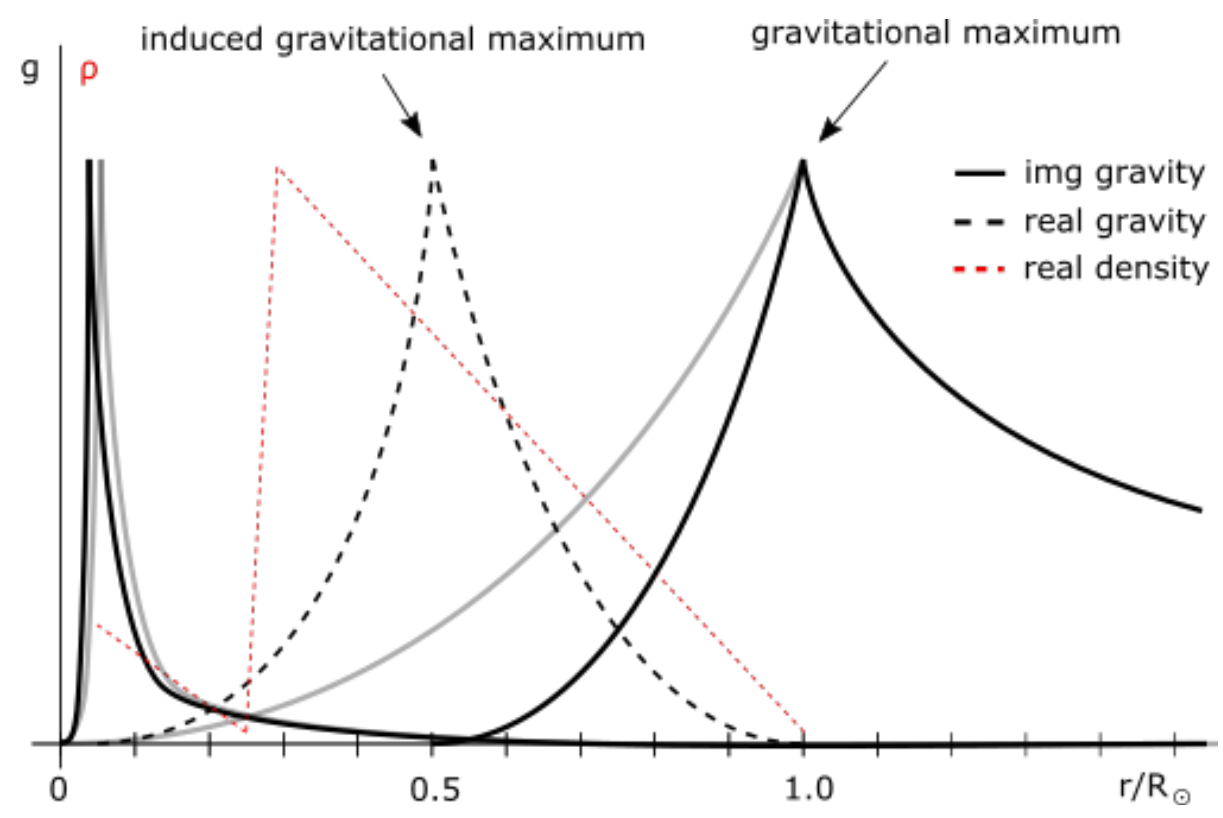

Fig. 1: Gravity of the Sun

Without the inner maximum, any free-falling real mass would be concentrated around the surface maximum. With inner maximums, concentration of real mass begins at the center.

However, as each inner maximum has lower capacity than its outer maximum, greatest density of real mass will not be at the center. Once inner maximum is at full capacity, as real mass accumulates between the inner maximum and the outer maximum, its counteracting the gravity of the outer maximum.

In equilibrium thus, greatest density of real mass is not at the outer maximum, rather between the inner and outer maximum.

This is shown on Fig. 1. Here, dark matter gravity provided by [img] gravitational maximums is represented by solid black lines, while real gravity provided by real mass and its induced (effective) maximum is represented by dashed black lines. In case of outer maximum, gray line represents gravity with no real mass acquired (naked maximum), while for inner maximum, it represents the initial core maximum. Red dashed lines show linearly approximated density of real mass.

From Fig. 1 one can extrapolate discontinuity candidates $\left(r / R_{\odot}\right): 0.0385 \approx 2 / 5 * 1 / 10,2 / 3 * 1 / 10$ (initial core maximum), 1/5, 1/4, 0.286, 1/2, 2/3, 3/4, 1 .

Note that there should be two major charge radii inside the Sun, if the outer charge is located at tachocline, and charge radii are mirrored relative to the induced real maximum, other charge radius boundary should be at $2 / 5 \mathbf{R}_{\odot}$ (mirroring the $2 / 3 \mathbf{R}_{\odot}$ boundary). 
In addition to these, there are other candidates, representing maximum deviation from these values - ie. discarding CMB relative kinetic energy, rest surface maximum is at $0.94 \mathbf{R}_{\odot}$.

\subsection{Energy replenishment}

Primary energy source of the Sun is, most likely, fusion.

Fuel for fusion must either be the real mass of the Sun (accumulated matter) or matter created through conversion of imaginary mass (dark gravitational potential) to real mass by some unknown mechanism.

In case of such conversion it would take tens of billions of years to spend all fuel.

However, this solution implies the Sun is eating itself and is highly unlikely.

The Sun is thus, most likely, burning its real mass which was accumulated during inflation of its maximums (whether through inflation of smaller maximums or acquisition of matter by increasing vacuum pressure).

When compared to other living beings, it would be reasonable to assume that Sun has a relatively constant real rest (constitutional) mass and an amount of fuel which is being cyclically replenished.

To determine how much fuel the Sun has left it is necessary to determine how much fuel it had at the beginning and the rate of fuel consumption.

Assuming fusion reaction $4 \mathrm{H}->\mathrm{He}$ (energy per reaction $\mathbf{E}_{\mathbf{r}}=4.32$ * $10^{-12} \mathrm{~J}$ ) and power output $\mathbf{P}$ of 3.8 * $10^{26} \mathrm{~J} / \mathrm{s}$, time needed to spend all fusion fuel is:

$$
\Delta t=\frac{m}{m_{p}} * \frac{E_{r}}{4} * \frac{1}{P} * N
$$

$\mathrm{m}=$ available mass

$\mathrm{m}_{\mathrm{p}}=$ proton mass

$\mathbf{E}_{\mathrm{r}}=$ energy per reaction

$P=$ power output

$\mathrm{N}=$ fraction of mass used in fusion

Since the Sun has two [major] maximums, fusion may be occurring at two places - in the core and above the core.

Gravitational mass of the surface maximum is known to be $1.988500 * 10^{30} \mathrm{~kg}$, while the gravitational 
mass of the core has been calculated here to be $2.951797 * 10^{27} \mathrm{~kg}$.

Assuming that the calculated mass is the mass of the maximum and therefor equal to the internal capacity for real mass, this capacity in equilibrium should be full and, due to mass loss (ie. through radiation), excess real mass must be constantly (cyclically) consumed as fuel.

Note that calculated mass implies such density of the core that temperature should be orders of magnitude higher than current assumptions, for thermonuclear fusion to occur.

If fusion is occurring in the core, most likely it is not thermonuclear.

It has also been hypothesized that the ratio of core mass and surface mass should be correlated with the ratio of mass between inner and outer planets.

Assuming that at the beginning of the core feeding cycle, these ratios are equal, fuel mass is the excess mass in the outer core corresponding to the ratio.

In case of thermonuclear fusion and with $2 / 3$ of mass consumed, time needed for the core to spend all fuel is:

$$
\begin{gathered}
\Delta t=\frac{m}{m_{p}} * \frac{E_{r}}{4} * \frac{1}{P} * N=\frac{8.90211033 * 10^{27} \mathrm{~kg}}{1.67265 * 10^{-27} \mathrm{~kg}} * \frac{4.32 * 10^{-12} \mathrm{~J}}{4} * \frac{1}{3.8 * 10^{26} \frac{\mathrm{J}}{\mathrm{s}}} * \frac{2}{3} \\
\Delta t=10084091956967735 \mathrm{~s}=319545591.5 \text { years }
\end{gathered}
$$

where $\mathrm{m}=\mathbf{8 . 9 0 2 1 1 0 3 3} * 10^{27} \mathrm{~kg}$ is the previously calculated initial mass of the core.

Assuming that, at the start of consumption cycle, imaginary mass (gravitational maximum) grows to initial mass radius $\left(\mathbf{0 . 2 8 6} \mathbf{R}_{\odot}\right)$ and decreases with energy loss, time left (assuming constant rate of consumption) before the next feeding cycle is then:

$$
\begin{gathered}
t=\left(2.951797 * 10^{27}-\frac{1}{3} 8.90211033 * 10^{27}\right) * \frac{3}{2} \frac{1}{8.90211033 * 10^{27}} \Delta t \\
t=-26461406017707 s=-838511.4 \text { years }
\end{gathered}
$$

Negative time may be interpreted as the next cycle being overdue (core spent all fuel 838k years ago and is currently burning constitutional mass), or, that more than $2 / 3$ of mass must be consumed in fusion.

In case $\mathbf{7 0} \%$ of mass may be spent: 


$$
\begin{gathered}
\Delta t=10588296554816122 \mathrm{~s}=335522871 \text { years } \\
t=1114734114271587 \mathrm{~s}=35323792.5 \text { years }
\end{gathered}
$$

However, as stated already, thermonuclear fusion in the core is unlikely. In case there is no fusion in the core at all, ruling out standard chemical reactions and radioactivity, the remaining possibility is heat generation through gravitational (Kelvin-Helmholtz) contraction:

$$
\begin{aligned}
& \frac{d U_{r}}{d t}=\frac{-3 G M_{i}^{2}}{10 R_{i}^{2}} \frac{d R}{d t} \\
& \mathbf{M}_{\mathrm{i}}=\text { initial core mass }=8.90211033 * 10^{27} \mathrm{~kg} \\
& \mathbf{R}_{\mathrm{i}}=\text { initial core radius }=\mathbf{0 . 2 8 6} \mathbf{R}_{\odot}=198970200 \mathrm{~m}
\end{aligned}
$$

assuming logarithmic relationship between mass and radius contraction, the contraction may be approximated from the rate of Jupiter contraction:

$$
\begin{array}{r}
\frac{d R}{d t}=\frac{10^{\frac{M_{i}}{M_{J}}}}{3^{\frac{R_{i}}{R_{J}}}} \frac{d R_{J}}{d t}=-7.29401291 * 10^{-8} \frac{\mathrm{m}}{\mathrm{s}} \\
\mathbf{M}_{\mathbf{J}}=\mathbf{J} \text { upiter mass }=1.89819 * 10^{27} \mathrm{~kg} \\
\mathbf{R}_{\mathbf{J}}=\text { Jupiter radius }=71492000 \mathrm{~m} \\
\mathrm{~d} \mathbf{R}_{\mathbf{J}} / \mathrm{dt}=\text { rate of Jupiter contraction }=-3.17 * 10^{-11} \mathrm{~m} / \mathrm{s}
\end{array}
$$

giving energy radiation of:

$$
\frac{d U_{r}}{d t}=2.9233705 * 10^{21} \frac{J}{s}
$$

and time to spend all fuel:

$$
\Delta t=\frac{3 G\left(M_{i}-M\right)^{2}}{10 R_{i}}\left(\frac{d U_{r}}{d t}\right)^{-1}=1218751736351319 \mathrm{~s}=38619912 \text { years }
$$

$$
M=\text { current core mass }=2.951797 * 10^{27} \mathrm{~kg}
$$

From this one can calculate the core radius at the end of the cycle (all fuel spent):

$$
R=R_{i}-\Delta t \frac{d R}{d t}=R_{i}-\left(M_{i}-M\right)^{2} \frac{R_{i}}{M_{i}^{2}}=0.158221 R_{\odot}=110074291 \mathrm{~m}
$$

$$
\mathbf{R}_{\odot}=\text { Sun surface radius }=695700000 \mathrm{~m}
$$


With current core radius at $\mathbf{0 . 2} \mathbf{R}_{\odot}$, amount of fuel left is:

$$
\frac{0.2-0.158221}{0.286-0.158221}=0.327 \approx \frac{1}{3}
$$

It is unlikely though that all fuel is spent during the cycle, total amount spent is most likely equal to $2 / 3$ (equivalent with fusion), in which case the cycle period is:

$$
\Delta t_{r e}=\frac{2}{3} \Delta t=25746608 \text { years }
$$

and the core is at the end of a cycle.

The obtained core cycle period agrees well with the hypothesized 2 nd order cycle period of the Solar System ( $\approx 26$ million years).

Since the 2 nd order cycle period is also equal to periodicity of impacts and extinctions on Earth and other planets, all these Solar events are likely synchronized - once the core fuel is exhausted, additional fuel is provided by the outer half of the Sun at the same time equal quantity of its own fuel is replaced with mass from impactors.

Gravitational stress may even create wormholes through core/surface sunspots enabling direct consumption of impactor mass by the core.

Note that, with core radius oscillation, its time independent radius is obtained from the volumetric superposition of $\mathbf{0 . 2} \mathbf{R}_{\odot}$ and $\mathbf{0 . 2 8 6} \mathbf{R}_{\odot}$ cores:

$$
\begin{gathered}
\frac{4}{3} \pi R^{3}-\frac{4}{3} \pi R_{c}{ }^{3}=\frac{4}{3} \pi R_{i}{ }^{3}-\frac{4}{3} \pi R^{3} \\
R^{3}=\frac{{R_{i}{ }^{3}+R_{c}{ }^{3}}_{2}^{2}=\frac{\left(0.286^{3}+0.2^{3}\right) R_{\odot}{ }^{3}}{2}}{R=\sqrt[3]{\frac{\left(0.286^{3}+0.2^{3}\right)}{2}} R_{\odot}=0.25 R_{\odot}=\frac{1}{4} R_{\odot}}
\end{gathered}
$$

Such oscillation must be present on standard scale too - thus, all results obtained from measurements of nuclear observables may be understood as superpositions in time and/or space, however, in reality these are not constants, rather statistical mean state of changing phenomena.

Regardless of scale, no equally evolved (identical) phenomena can exist at two points in time, nor can they exist at multiple points in space. De-localization may seem possible through stretching of [a point in] space/time, however, this is fragmenting (quantizing) the phenomena and its space. Even if it remains 
strongly entangled, it is never, as a whole, at multiple points in space/time, although, with energy applied, de-localized space may collapse to one of the fragmented points.

Unlike the core, the outer part of the Sun is most likely powered by fusion.

However, it too must have constitutional mass and fuel mass fraction of real mass (excess mass).

Most likely, fuel mass is equal to the previously calculated kinetic energy (CMB relative) of the Sun. In that case, time to spend the fuel is:

$$
\begin{gathered}
\Delta t=\frac{m}{m_{p}} * \frac{E_{r}}{4} * \frac{1}{P} * N=\frac{1.18437729 * 10^{29} \mathrm{~kg}}{1.67265 * 10^{-27} \mathrm{~kg}} * \frac{4.32 * 10^{-12} \mathrm{~J}}{4} * \frac{1}{3.8 * 10^{26} \frac{\mathrm{J}}{\mathrm{s}}} * \frac{2}{3} \\
\Delta t=4.25 * 10^{9} \text { years }
\end{gathered}
$$

The value is in agreement with the hypothesized 1st order cycle and it is likely equal to previously calculated real age of the Earth $\left(4.29 \pm 0.05 * 10^{9}\right.$ years $)$, suggesting the Solar System is at the end of the 1 st order cycle.

Note that the calculated age is exactly $1 / 3$ of the obtained age of the observable universe in one class of measurements (Lensedquasars/Near) - $12.75 * 10^{9}$ years (also in agreement with more recent bTFR measurements ${ }^{60}$ ), supporting the cycling hypothesis (this would be the end of a 3rd cycle).

Gravitational stress of the 1 st order must be order(s) of magnitude larger than that of the 2 nd order.

Likely, at the end of such cycle, Sun briefly looses some momentum (relative to CMB) with the spin change of the outer maximum. It falls into a lower energy level, closer to the galactic center. Afterwards, it starts expanding again consuming hydrogen fuel as it returns to the current state again.

Note that a reason for discrepancy in measurements of the age of the universe (Hubble constant) could be the same as in the case of the age of Earth. I have previously hypothesized cyclic time compression (evolution inflation, due to gravitational stress), with coupled periods of $\mathbf{1 . 5 1 2}$ and $\approx \mathbf{2 6}$ million years. With the next larger period being $T_{u}=\mathbf{4 . 2 5} \mathrm{Gy}$, its time compression should be:

$$
\Delta t_{c_{u}}=\frac{\Delta t_{c_{x}}}{T_{x}} T_{u}=\frac{24751.794 y}{1512000 y} 4.25 * 10^{9} y=69573495.04 \text { years }
$$

where $\Delta t_{C_{x}}$ is the previously calculated compression of time with a single Tx (1512000 years) pulse. 
Now one can calculate how much overestimated is the currently accepted age of the observable universe $T_{\text {img }}=13.799 \pm 0.021 * 10^{9}$ years:

$$
\sigma_{T_{i m g}}=\left\lfloor\frac{T_{i m g}}{T_{u}}\right\rfloor\left[\Delta t_{c_{u}}+\left(\Delta T_{E_{i m g}}-T_{u}\right)\right]=1.07872048512 \pm 0.05 * 10^{9} \text { years }
$$

where $\Delta \mathrm{T}_{\mathrm{E}_{\text {img }}}\left(4.54 \pm 0.05 * 10^{9}\right.$ years $)$ is the currently accepted age of Earth.

This gives for the real age of the universe:

$$
T=T_{i m g}-\sigma_{T_{i m g}}=12.72027951488 \pm 0.071 * 10^{9} \text { years }
$$

resolving the discrepancy.

Another interesting solution is obtained if the fuel amount is equal to real mass of the Sun calculated with the assumption of, across Solar System, invariant, real $\hbar_{\mathrm{mg}}$ constant:

$$
m=\frac{\hbar_{m g}}{g}=\frac{6.968267285 * 10^{20} \mathrm{~N}}{274 \mathrm{~m} / \mathrm{s}^{2}}=2.543163243 * 10^{18} \mathrm{~kg}
$$

For $N=2 / 3$ (here, the other $1 / 3$ would be the solar wind), time needed to spend this fuel is:

$$
\begin{gathered}
\Delta t=\frac{m}{m_{p}} * \frac{E_{r}}{4} * \frac{1}{P} * N=\frac{2.543163243 * 10^{18} \mathrm{~kg}}{1.67265 * 10^{-27} \mathrm{~kg}} * \frac{4.32 * 10^{-12} \mathrm{~J}}{4} * \frac{1}{3.8 * 10^{26} \frac{\mathrm{J}}{\mathrm{s}}} * \frac{2}{3} \\
\Delta t=\frac{2}{3} * 4321249.297 \mathrm{~s}=33.3 \text { days }
\end{gathered}
$$

For $\mathbf{N}=1 / 2$ :

$$
\Delta t=\frac{1}{2} * 4321249.297 s=25 \text { days }
$$

This solution is not plausible as it requires continuous hydrogen uptake from interstellar medium. While charged protons and electrons are absorbed at Sun's poles and could be combined to form hydrogen at the center (assuming the Sun is not ideally neutral and has gravitational holes at poles - at least periodically opened, although the charges could also be inefficiently transfered inside as electric current), energy bandwidth is not sufficient to power the Sun.

Interestingly, the solution (with $\mathbf{N}=\mathbf{2 / 3}$ ) is close to the polar rotation period of the Sun ( $=\mathbf{1 / 2}$ gives equatorial period) where the uptake would happen. 
However, although unlikely in a stable state, this is likely the feeding method in the previous hypothesis $\left(4.25 * 10^{9}\right.$ years cycle). Once the spin momentum collapses into a two-dimensional form, the Sun will be extremely charged. With an extremely strong non-homogeneous magnetic field it would be able to acquire required mass efficiently and quickly.

Differential rotation of the Sun could be a fossilized evidence of spin collapse, suggesting it breaks into multiple quanta in the form of concentric rings (oppositely charged rings must have anti-aligned spin to conserve the magnetic field).

Such fossil is perhaps more evident on Jupiter, where wind velocities are correlated with gravity.

The extremely stable and static cyclones on Jupiter's poles indicate that it might have small gravitational holes open today.

However, if these are open, small gravitational gaps or indentations should also exist between layers associated with each ring quanta. Strong magnetic field and measurements of gravity do support this theory, although the indentations would have to be extremely small - if gravitational disturbances are not due to standard $\left(\mathbf{U}_{\mathbf{0}}\right)$ scale matter, as currently interpreted (in which case they would be the fossil of the healing process).
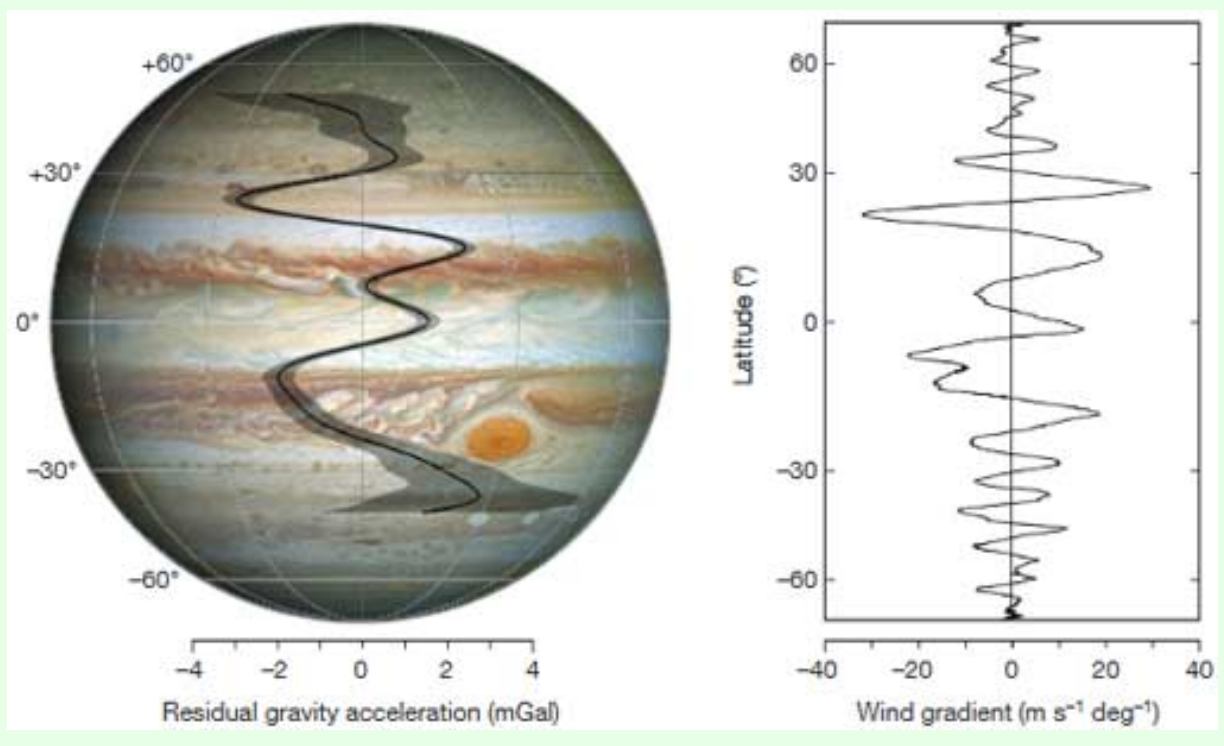

Fig. 1: Jupiter gravity disturbances and wind gradient ${ }^{61}$

The cells of all living species are regenerating on a periodic basis, for example, 1/3 of hippocampal neurons in humans and mice is exchanged during the lifetime ${ }^{62}$, thus, the cellular regeneration in the Sun should not be surprising, whether it is food or constitutional mass. 
Capacity for real mass below the Sun's surface may be full, but all mass orbiting the Sun may be considered as its real mass.

However, it is obviously not fuel mass, rather constitutional or symbiotic mass.

The 3rd order period of the Solar System cycle may be related to this mass through the mass barycenter of the system.

I have previously calculated the neutral gravitational mass equivalent for the surface plasma at the equator which would make its angular velocity Keplerian.

The source for this energy may be the motion of the barycenter.

In any case, if one assumes that conversion between neutral and electro-magnetic component of the general force of the Sun is also periodic and that such energy replaces fusion reactions in equivalent way, the period of recharge is:

$$
\begin{gathered}
\Delta t=\frac{m_{r e}}{m_{p}} * \frac{E_{r}}{4} * \frac{1}{P} * N=\frac{4.042341 * 10^{25} \mathrm{~kg}}{1.67265 * 10^{-27} \mathrm{~kg}} * \frac{4.32 * 10^{-12} \mathrm{~J}}{4} * \frac{1}{3.8 * 10^{26} \frac{\mathrm{J}}{\mathrm{s}}} * \frac{2}{3} \\
\Delta t=45790644230537 \mathrm{~s}=1451018 \text { years }
\end{gathered}
$$

and it is in good agreement with the hypothesized 3rd cycle period (a fraction of mass $\mathbf{N}=\mathbf{0 . 6 9 4 6 8 4 7}$ would yield the hypothesized value - 1512000 years).

In comparison with living beings, one might notice a problem of exhausted fuel - what happens with the ash from fusion reactions (end products of fusion)?

There are couple of solutions:

1. the ash is ejected periodically,

2. the ash forms the constitutional mass.

Time compression at the end of Solar System cycles implies gravitational stress of Solar System maximums.

While the 2nd hypothesis might be plausible during initial formation of the Sun, at least at the end of one of the cycles some mass must be ejected out from the Sun.

It certainly seems easier than in case of planets, as unlike the planets, the Sun does not have a solid [real] mantle to block the explosion (the mantle of the Sun are the terrestrial planets, however, they are in 
collapsed form with plenty of space in between).

The ash content depends on the cycle period, being mostly Helium in smaller cycles but with heavier elements formed in explosions at the end of larger cycles.

A full collapse of the maximum is the collapse of a 3-dimensional spherical neutral form into 2-dimensional charged form. Since the surface maximum of the Sun is entangled with Mars' maximum, at the time of collapse, two ring maximums are aligned and the ejection of ash is not isotropic, rather targeting Mars.

At that point, both the Sun and Mars have a significant (extreme) magnetic field generated by charged maximums so Mars would likely attract ferromagnetic/charged ejecta from the Sun.

The evidence for this is the Fe covered surface of Mars.

Note that the collapse involves the change of spin of the maximums. First, the holes are opening on the poles of the spherical Sun maximum while the axial tilt starts increasing, the poles of the Sun and Mars are only briefly fully aligned before the equilibrium of stable spin states is reached.

Thus, most mass is ejected in the first and last moments of the spin change, through the equilibrium poles - out of the Solar System.

Note that the magnetic field is weakest at these times, as it increases, the momentum of particles is curved and aimed at Mars.

\subsection{As a living organ[ism]}

Considering the energy output (metabolic rate) of $\mathbf{P}=\mathbf{3 . 8} * \mathbf{1 0}^{26} \mathbf{W}$, the standard relation between metabolic rate and mass ${ }^{63}$ :

$$
\frac{P}{0.0484259259 \frac{d a y * W}{k c a l}}=70 * M^{\alpha}
$$

gives $\mathbf{0 . 8 6}$ for the $\boldsymbol{\alpha}$ exponent $\left(\mathbf{M}=\right.$ total mass of the sun $\left.=\mathbf{1 9 8 8 5 0 0} * \mathbf{1 0}^{\mathbf{2 4}} \mathrm{kg}\right)$. For a mammalian organ this would be between a kidney and a liver ${ }^{64}$, suggesting an embryonic stem cell in the process of differentiation. 


\section{Metabolism of Earth}

Transfer of energy in wild flora and fauna is generally balanced both horizontally and vertically.

Vertical transfer of energy is a part of metabolism but changes in horizontal current affect the vertical transfer too (and vice versa).

Humans dominate in both horizontal (surface to surface) and vertical (Sun - Earth interior) energy distribution and transformation, disrupting the harmonics of life.

Horizontal effect is the increasing number of individuals at the cost of decreasing number and diversity of other species, while vertically it is the unsustainable exploitation of radiated and stored resources of the Sun/Earth ecosystem.

Thus, one may interpret humans as the metabolism energy carrier particles, in a limited domain.

With a human population $\mathbf{N}$ of $7.674 * 10^{9}$, average mass $\mathrm{m}$ of $62 \mathrm{~kg}$, and average lifetime $\Delta \mathrm{t}$ of 72.6 years (data for year 2019, except mass - 2012):

$$
\begin{gathered}
P=\frac{N * m * c^{2}}{\Delta t}=\frac{7.674 * 10^{9} * 62 *\left(2.99792458 * 10^{8}\right)^{2}}{72.6 * 365.25 * 24 * 60 * 60}=1.86644116 * 10^{19} \mathrm{~W} \\
\frac{P}{0.0484259259 \frac{\text { day*W }}{\mathrm{kcal}}}=70 * M^{\alpha}=3.8542188 * 10^{20} \frac{\mathrm{kcal}}{\mathrm{day}}
\end{gathered}
$$

where $M$ is the mass of Earth $\left(5.9723 * 10^{24} \mathrm{~kg}\right)$.

This gives a value of $\mathbf{0 . 7 5 6}$ for $\boldsymbol{\alpha}$ exponent, in agreement with Kleiber's law.

However, in case of organ interpretation, the exponent suggests a superposition of a brain and a kidney.

Note that Earth has kidney [precursor] equivalents on surface.

In order for this superposition to differentiate into the brain, the exponent would have to reduce to 0.7 .

There are several ways to achieve that (sorted by probability, from highest to lowest):

1. increasing human lifetime $(\approx 25$ times) to 1813 years, 


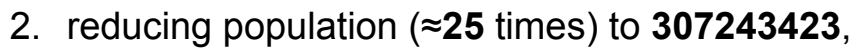

3. reducing mass ( $\approx 25$ times),

4. increasing Earth's mass $\approx 100$ times ( $\approx$ mass of Saturn).

If humans are indeed precursor proteins of neuron proteins of Earth, as carriers of energy of its brain metabolism, I would expect the solution to be a superposition of the above.

However, if Earth has a heart equivalent (core), most likely it also has a kidney equivalent. Thus, I would expect the population to differentiate into proteins of varying function.

I, strive for neutrality - the equal, balanced usage of all parts of my universe. I am aware though, that this is an unreachable singularity, but it is the journey that makes one alive - for without it there would be no senses, for a sense of reason, and a reason for existence. 


\subsection{Nature of human cells}

Dominance of lifeforms changes over time. At present time, homo species occupies and controls most of the surface of the planet. Human population is rising and thriving at the expense of other species.

While the dominion of species may be related to precursor nature of vital organism components, its behavior can be corrupted, so cultivation of new proteins becomes evolution of disease rather of something integral for survival.

While it is not questionable whether human species is a disease for the planet, it is questionable whether this is fatal or rather a normal part of evolution of healthy cells and proteins with self-correcting mechanisms.

Dividing the total surface area of Earth $\left(R=6371 * 10^{3} \mathrm{~m}\right)$ with the number of people, one gets the maximum size of the cell:

$$
A=\frac{4 \pi R^{2}}{7.7 * 10^{9}}=66242.13921 \mathrm{~m}^{2}
$$

Radius of space per person is:

$$
r=\sqrt{\frac{A}{\pi}}=145.2085665 \mathrm{~m}
$$

If the radius of a human occupied cell of Earth is the mean free path $\mathbf{r}$, the radius of a cell equivalent in human body of average diameter (height) $h=1.7 \mathrm{~m}$ is:

$$
r_{c}=\frac{r}{R} \frac{h}{2}=19.373298 * 10^{-6} m=19.373298 \mu m
$$

If one calculates using landmass only (people don't naturally live on water):

$$
\begin{gathered}
A=\frac{1.4894 * 10^{14} \mathrm{~m}^{2}}{7.7 * 10^{9}}=19342.85714 \mathrm{~m}^{2} \\
r=\sqrt{\frac{A}{\pi}}=78.46669775 \mathrm{~m} \\
r_{c}=\frac{r}{R} \frac{h}{2}=10.46879502 * 10^{-6} \mathrm{~m}=10.46879502 \mu \mathrm{m}
\end{gathered}
$$

Taking into account space used by wild flora and fauna:

$$
r=\frac{1}{2} \sqrt{A}=69.53930029 m
$$




$$
r_{c}=\frac{r}{R} \frac{h}{2}=9.277728025 * 10^{-6} m=9.277728025 \mu m
$$

This is in the range of a typical cancer cell. It is, of course, in the range of healthy cells too, but human cells are far from healthy.

It might seem that the radius $\mathbf{r}\left(\mathbf{r}_{\mathbf{c}}\right)$ changes with population, but this is not the case - if human space decreases, the space of wild flora and fauna increases and vice versa, thus it generally evolves weakly, remaining almost constant.

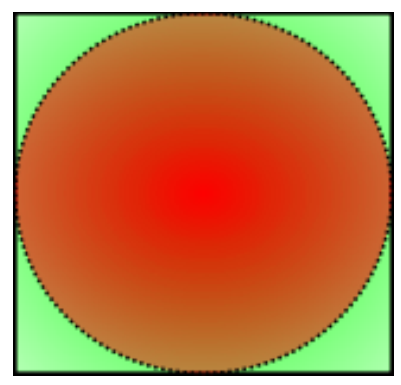

Fig. 1: Homo.beta cell

Fig. 1 shows the unit of space on Earth's surface, circled space (red) is occupied by a human and domesticated flora and fauna, other (green) by wild flora and fauna.

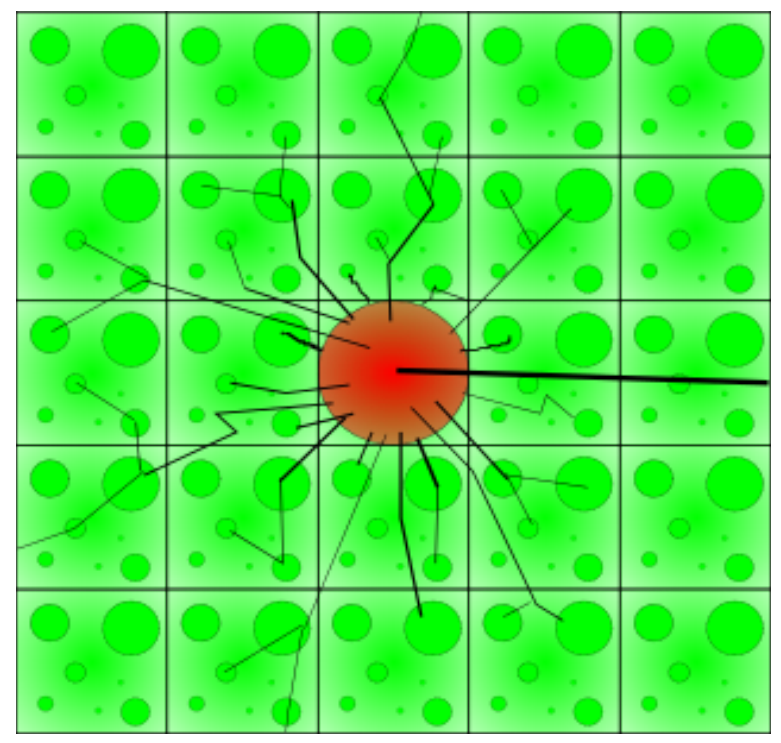

Fig. 2: Normal cells

Fig. 2 shows the normal (healthy) unit of space on Earth. Red is a cell of homo.sapiens (Earth's neuron cell), black lines are spiritual connections (synapses). 


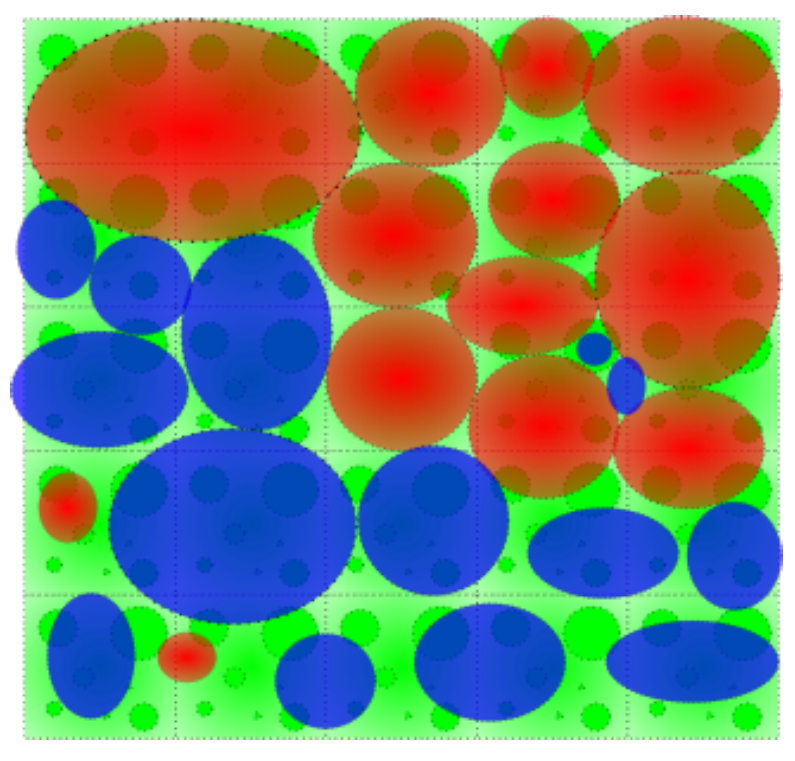

Fig. 3: Cancer cells

Fig. 3 shows the cancerous (current) unit of space on Earth. Blue and red are polarized human (cancer) cells.

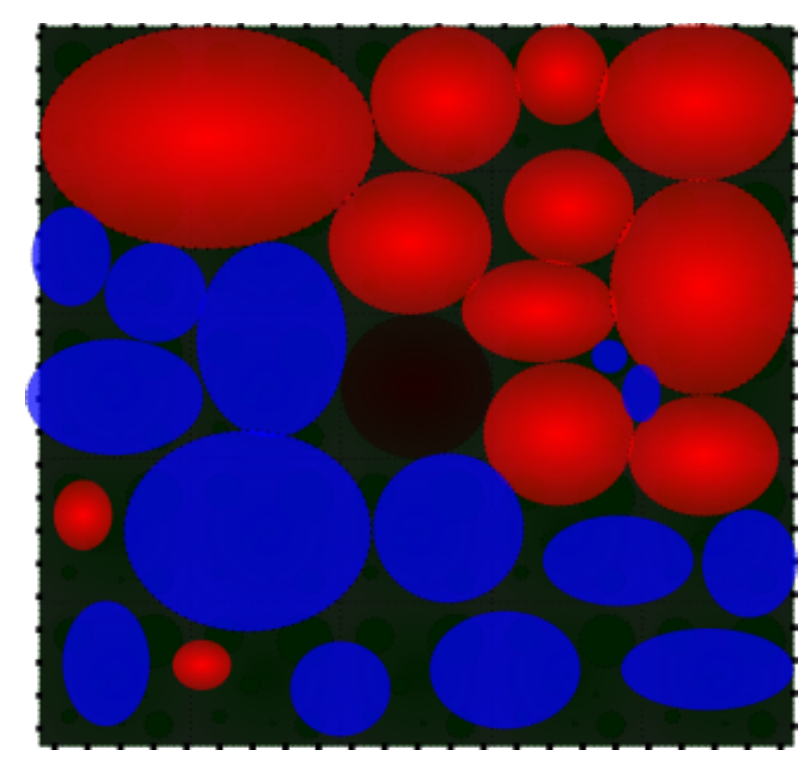

Fig. 4: Dead space

Fig. 4 shows the outcome - death.

Carbon footprint is not the issue. It is just a side-effect of the real issue - nature of the human footprint.

Cancer cell contains the individuals (proteins) and space affected by cancerous population, but one can even calculate the role of a human in the cancer cell: 


$$
\lambda=\frac{h}{2} \frac{1}{r} r_{c}=\frac{h}{2} \frac{1}{R} \frac{h}{2}=\frac{h^{2}}{4 R}=1.134044891 * 10^{-7} \mathrm{~m}
$$

This is in the range of a TGF- $\beta$ protein, a key player in cancer development.

Confirmation of this comes from recent studies ${ }^{65}$, revealing human nature of TGF- $\beta$ :

"And while it may be difficult to imagine a protein with two dramatically different faces, it may be even more difficult to contemplate cancer cells exhibiting traits, such as cunning and deception. But the research underway at the University of Basel, and collaborating laboratories, has revealed that TGF- $\boldsymbol{\beta}$ not only is a two-faced protein, it also is one that seems almost Machiavellian in its activities." 66

Cancerous TGF- $\boldsymbol{\beta}$ suppresses the immune response and prevents old cells/proteins from dying (regenerating). Humanity is, at the time of this writing, expressing this cancerous behavior on many levels:

- through treatment of diseases (including cancer) humanity is suppressing the immune system of Earth,

- forcing human life at all costs and treating death (as a disease) - instead of letting cells (and proteins people/animals) die as programmed so they can regenerate,

- treating Earth and other life forms (and, generally, even people) as resources - instead of living in a sustainable symbiotic relationship,

- creating and living in centralized, stressful environments, promoting inequality in wealth and health,

- denying the truth.

\section{Earth's cells are not fuel cells, they are living cells.}

The average cell cycle period of eukaryotic cell is $T_{0}=14.5$ hours, scaled to Earth size, it is:

$$
T_{1}=\sqrt{T_{0} * T_{x}}=50 \text { years }
$$

where $T_{X}$ is the period of $3 r d$ order existence cycle of Earth $\left(1.512 * 10^{6}\right.$ years). 


\section{The cycle of life and death}

An atom or a planetary system consists of relatively massive matter and relatively empty space of gravitational wells.

The energy of this space is in its vacuum proportional to its spin momentum and characterized by electric and magnetic permeability of polarized quanta of certain scale.

Gravitational wells (souls) are not intrinsically coupled with matter - otherwise, there would be no death.

All souls thus oscillate between different bodies.

This oscillation can be vertical (between different scales) or horizontal (between species of the same element, such as carbon), although even horizontal oscillation includes a temporary scale inflation/deflation between stable states.

Species in horizontal oscillation have comparable lifetimes so gravitational collapse generally indicates a permanent decoupling of particular soul/matter pair (death).

Primary (prevalent) oscillation type depends on pressure/temperature of the environment.

Man's desire to extend his dying baffles all of common sense. Why would one not want to leave the aging body and start anew? It seems, it is in nature of polarized to keep patching the patches of the aging systems instead of letting things die and recycle in peaceful honor.

It is alas, the fear of death, unjustly implanted into the seeds of man by man that makes him a zombie, sad and cancerous, even at times of abundant life. 


\section{Dark energy}

If planetary systems are [condensed] atoms, observable universe becomes a gas of extremely low density. Dark energy, if it exists, is thus simply the energy of gas expansion due to scaled pressure/temperature change. Such expansion requires exponentially increasing amount of energy, cannot continue forever, and at this state of the observable universe, it is more likely to be decelerating, rather than accelerating.

Galaxies are simply quantum vortices.

Black holes and other gravitational wells of $U_{1}$ scale can be understood then as vacuum quanta, increasing in strength with expansion and causing contraction of matter, with stretched space between them creating (inflating) new gravitational wells between galaxies. This is the reason of exponential growth of energy requirement.

The expansion of the universe and its metric has been questioned before ${ }^{67}$ and results are consistent with a non-expanding, Euclidean universe, although the increase in redshift with distance was not explained.

Recent evidence shows that the expansion of the observable universe is not accelerating ${ }^{68}$ and the redshift previously used as evidence for acceleration should be attributed to local "bulk flow" instead.

The fact that photon has a mass and the existence of energy absorbers of lower scale explains the redshift increase with distance. It should be noted also that, due to carrier mass, forces of nature change over time, splitting in strong evolution events and evolving slowly during weak evolution. 


\section{Stability of elements}

Structure of $U_{0}$ elements is entangled with the configuration of $U_{1}$ universe. This also makes the stability of isotopes dependent on this configuration.

The stability curve and decay rates of individual isotopes thus change strongly in transition from one cycle state to another, but also oscillate during state lifetime.

Stable isotopes are concentrated along this curve:

$$
\begin{gathered}
N(P, t)=\left\lfloor P *\left[1+\left(\frac{N_{\max }}{P_{\max }}-1\right) * \frac{P}{P_{\max }}\right]+\sigma_{T}\right] \\
\sigma_{T}=\left[-\left(C_{1} * C_{2}\right) *\left(\frac{C_{2}}{C_{1}}-1\right)+\left(C_{2}-C_{1}\right) * \frac{t}{\Delta_{t}} *\left(C_{1}+C_{2}\right)\right] * \frac{P}{P_{\text {max }}} \\
\sigma_{T}=\left[\left(C_{1} * C_{2}-C_{2}{ }^{2}\right)+\left(C_{2}{ }^{2}-C_{1}{ }^{2}\right) * \frac{t}{\Delta_{t}}\right] * \frac{P}{P_{\max }}
\end{gathered}
$$

where $\mathbf{N}=\mathbf{N}_{\mathbf{0}}$ is the number of neutrons, $\mathbf{P}=\mathbf{P}_{\mathbf{0}}=\mathbf{Z}$ is the number of protons of the isotope and $\mathbf{P}_{\max }$ is the maximum number of protons for a stable element (for the Solar and equivalent systems, $\mathbf{P}_{\max }=\mathbf{8 2}$, corresponding to $\mathbf{P b}$ - lead).

$\sigma_{\mathbf{T}}$ is the small shift in value of $\mathbf{N}$ due to weak evolution through state lifetime $\left(\boldsymbol{\Delta}_{\mathbf{t}}\right)$.

$$
\begin{gathered}
\frac{P_{\max }}{N_{\max }}=\frac{N_{1}}{P_{1}} \\
P_{P_{\max } / N_{\max }}=\left\lfloor E H_{N_{1} / P_{1}}\left(P_{s}, N_{\left.P_{\max } / N_{\max }\right)}\right\rfloor\right.
\end{gathered}
$$

where $\mathbf{N}_{\mathbf{1}}$ is the number of neutrons and $\mathbf{P}_{\mathbf{1}}$ the number of protons of the parent system $-\mathbf{U}_{\mathbf{1}}$.

$\mathbf{P}_{\mathrm{S}}$ is the atomic number (number of protons) of the most stable element - element with maximum number of stable isotopes.

$\mathbf{P}_{\mathbf{P}_{\text {max }} / \mathbf{N}_{\text {max }}}$ is the atomic number of the element lying on the $N(P, t)$ curve with $P / N$ ratio equal to $\mathbf{P}_{\text {max }} / \mathbf{N}_{\text {max }}$

For the Solar System, in state 6p4n: 


$$
\begin{gathered}
\Delta_{t}=1.51 * 10^{6} \text { years } \\
\frac{P_{\max }}{N_{\max }}=\frac{2}{3} \\
P_{2 / 3}=\left\lfloor E H_{4 / 6}\left(P_{s}, N_{2 / 3}\right)\right\rceil \\
C_{1}=2, C_{2}=3
\end{gathered}
$$

Note that the constants $\mathbf{C}_{1}$ and $\mathbf{C}_{2}$ are the same as those determined in chapter "Earth, as a living organ[ism] - Age and 3rd order period - Speed of time".

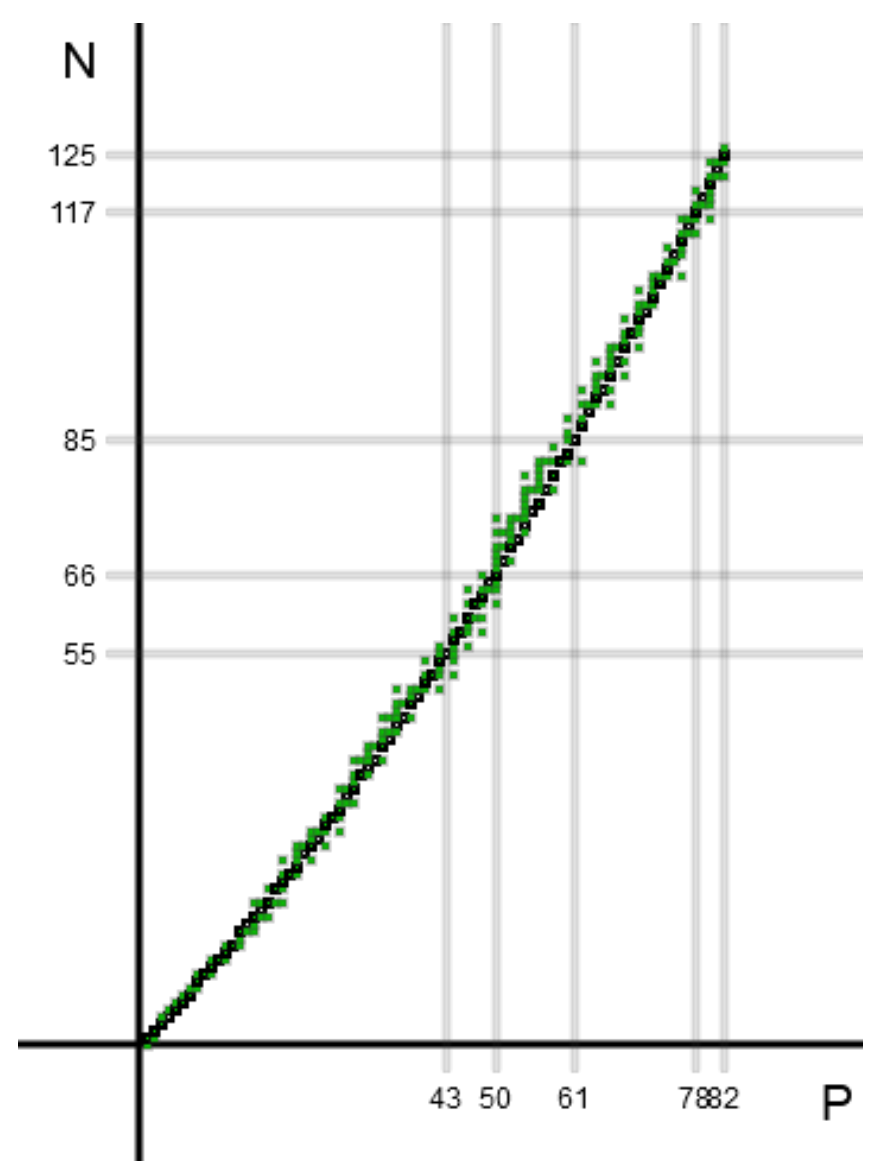

Fig. 1: Stable isotopes of the Solar System in state $6 p 4 n$ at $t>1495840$ years

Fig. 1 shows all stable isotopes of the Solar System (green) and the N(P,t) curve (black).

Note the following:

- for $t>1495840$ years $\left(t \approx \Delta_{t}\right)$, the isotope lying on the curve with $P / N$ ratio exactly equal to $2 / 3$ is $P t-195$ (Platinum, $\mathbf{P}=\mathbf{7 8}$ ). The placement of other Platinum isotopes is symmetric relative to the curve, 
- for $\sigma_{\mathbf{T}}=\mathbf{0}\left(\mathbf{t}=\mathbf{3 / 5} \Delta_{\mathrm{t}}\right)$, the $\mathbf{P}_{\mathbf{2} / 3}$ isotope is $\mathbf{P b - 2 0 5}$ (Lead, $\mathbf{P}=\mathbf{8 2}$ ). At $\mathbf{t}=\mathbf{3 / 5} \Delta_{t}$ this was a stable isotope. $1 / 3$ of other stable isotopes are above the curve, $2 / 3$ below,

- for $t=4 / 5 \Delta_{t}$ the $P_{2 / 3}$ isotope is $\mathbf{H g - 2 0 0}$ (Mercury, $P=80$ ). 1/3 of other stable isotopes are above the curve, $2 / 3$ below,

- the ratio of horizontal to vertical distance between Lead-205 and Platinum-195 is (82 - 78) / (123 - 117) $=4 / 6=2 / 3$,

- the ratio of horizontal to vertical distance between Lead-205 and $\mathbf{H g}-\mathbf{2 0 0}$ is $(\mathbf{8 0}-\mathbf{7 8}) /(\mathbf{1 2 0}-\mathbf{1 1 7})=\mathbf{2 / 3}$,

- at $\mathbf{t} \approx \Delta_{\mathbf{t}}$, Tin (Sn, $\left.\mathbf{P}=\mathbf{5 0}\right)$ has the highest number of stable isotopes (10). Tin isotope lying on the curve is $\mathbf{S n - 1 1 6}$ (50 protons, 66 neutrons). 2/3 of other stable Tin isotopes is above the curve, 1/3 is below,

- at $\mathbf{t} \approx \boldsymbol{\Delta}_{\mathbf{t}}$, the only elements without stable isotopes are Tc (Technetium, $\mathbf{P}=\mathbf{4 3}$ ) and Pm (Promethium, $P=61)$. The isotopes lying on the curve are Tc-98 and Pm-146. Vertical distance from Sn-116 to Tc-98 is equal to horizontal distance from $\mathrm{Sn-116}$ to $\mathrm{Pm}-146$. 


\section{Electric gravity}

Electric force is a polarized component of the general force.

Inside the atom, force field between negative and positive charges is neutralized and electro-magnetic potential may be exchanged with gravitational potential.

Thus, a Hill sphere radius $\left(\mathbf{r}_{\mathbf{H}}\right)$ of an atom should be correlated with its charge radius.

$$
r_{H}=R \sqrt[3]{\frac{m}{3 M}}
$$

This gives, for Carbon-12 atom with nucleus mass $m=1.992646883 * 10^{-26} \mathrm{~kg}$ inside the gravity field of Earth at $\mathbf{R}=6371 \mathrm{~km}$ (surface):

$$
r_{H}=66 * 10^{-12} m=66 p m
$$

This is in agreement with experimentally obtained radius of $\mathbf{7 0 ~ p m ~}( \pm \mathbf{5 m})$. Calculation for other elements of the periodic table yields similar results.

Note that Hill radius is different for different isotopes of the same element while experimentally obtained atomic radii are charge radii and thus independent of the number of neutrons (radius represents the orbit of the outermost electron). In example, for Carbon-14 the obtained value is $69.5 * 10^{-12} \mathrm{~m}$, and even closer to $70 \mathrm{pm}$ if one calculates using equatorial radius of Earth instead of mean volumetric (a possible indicator that the Solar System soul was a part of a ${ }^{14}(\mathrm{C}-\mathrm{N}-\mathrm{O})$ cycle in previous incarnation).
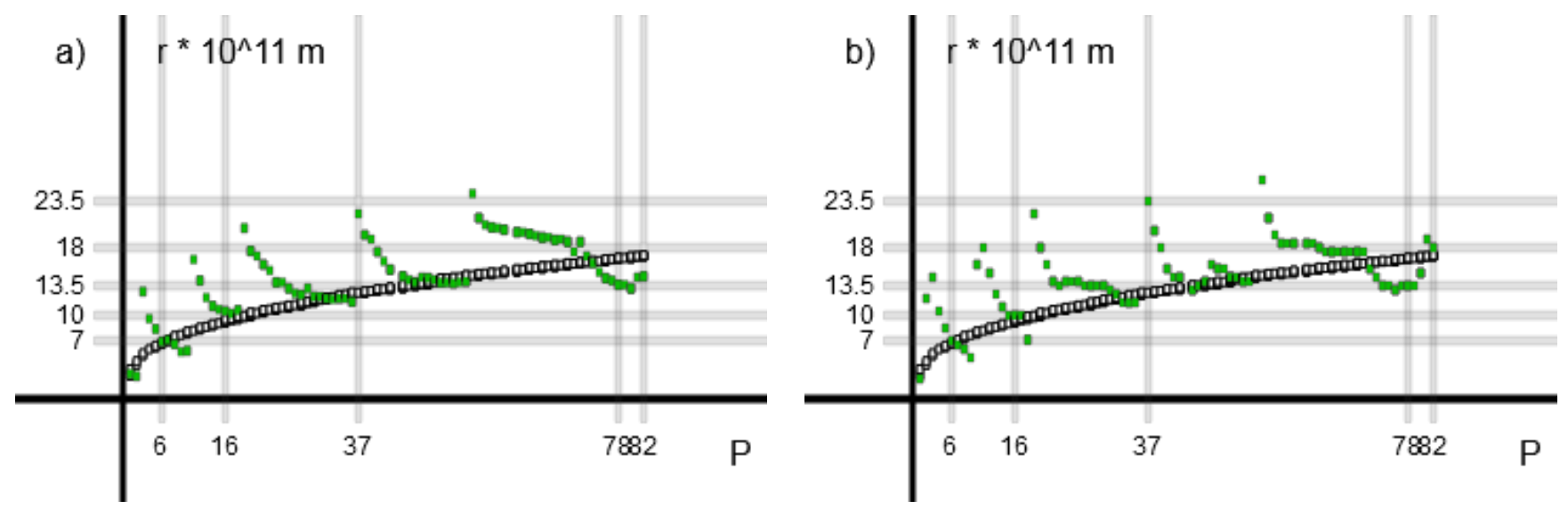

Fig. 1: Calculated Hill sphere and measured radius for stable isotopes: a) data from 2008. b) data from 1964.

Fig. 1 shows experimentally obtained radius (green) and calculated Hill sphere at $\mathbf{R}=\mathbf{6 3 7 1} \mathrm{km}$ (black) for all stable isotopes. Evidently, radii are not only correlated but values of covalent radii oscillate around the Hill radii, confirming the entanglement of $U_{0}$ and $U_{1}$. 
Comparing data from $1964 .{ }^{69}$ and $2008 .{ }^{70}$ shows a compression of radii and convergence to Hill radii such changes are expected in CR (no constants) and these should be accelerating as the Solar System approaches the end of the current state $(6 p 4 n)$.

In the intermediate state $(5 p 5 n)$ charges may be completely neutralized, and the radii of all elements may converge to Hill radius.
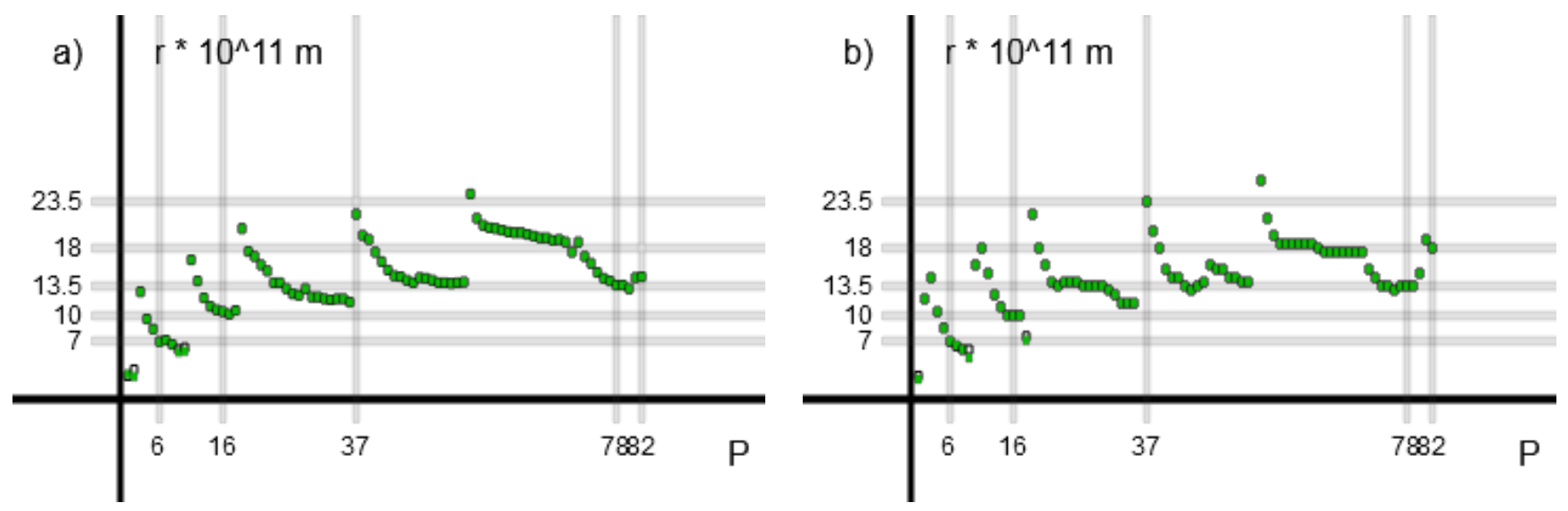

Fig. 2: Calculated Hill sphere (adjusted) and measured radius for stable isotopes: a) data from 2008. b) data from 1964.

Fig. 2 shows the experimentally obtained radius (green) and calculated Hill sphere at $\mathbf{R}=\mathbf{6 3 7 1} \mathrm{km}$ (black) for isotopes with neutron number adjusted to match the charge radius.

In calculations above, atomic mass has been quantized by $\mathbf{u}=1.66053907 * 10^{-27} \mathrm{~kg}$ (atomic mass constant) with integer number of protons $\mathbf{P}$ and neutrons $\mathbf{N}\left[\mathbf{m}=(P+N){ }^{*} \mathbf{u}\right]$ so Hill radii are quantized too. The overlap of Hill radii with charge radii in Fig. $\mathbf{2}$ shows that charge radius is quantized too (there is a number of neutrons $\mathbf{N}$ for which the Hill radius will match the charge radius). 

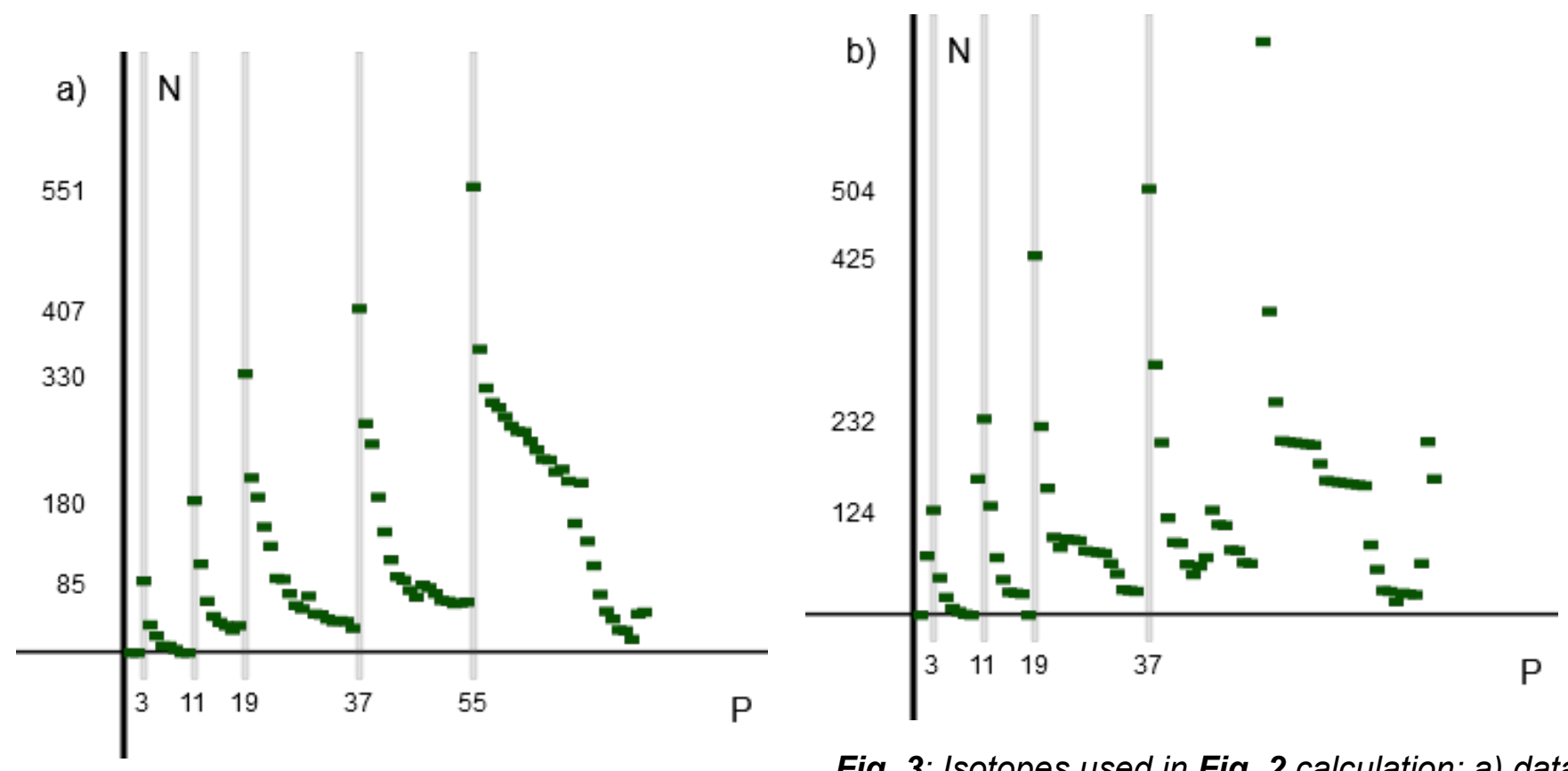

Fig. 3: Isotopes used in Fig. 2 calculation: a) data from 2008. b) data from 1964.

Fig. 3 shows the number of neutrons $\mathbf{N}$ used with each element to obtain Hill radius equal to charge radius.

From above figures it is obvious that elements (atoms) are grouped into shells the same way as electrons are grouped in atoms.

\begin{tabular}{|l|l|l|l|l|}
\hline $\mathbf{n}$ & shell (alt shell) & entanglement & elements & total elements $=\mathbf{2 n}^{2}$ \\
\hline 1 & $\mathrm{~K}$ & - & $1-2(\mathrm{H}-\mathrm{He})$ & 2 \\
\hline 2 & $\mathrm{~L}$ & $\mathrm{Q}$ & $3-10(\mathrm{Li}-\mathrm{Ne})$ & 8 \\
\hline 2 & $\mathrm{~L}(\mathrm{Q})$ & $\mathrm{L}$ & $11-18(\mathrm{Na}-\mathrm{Ar})$ & 8 \\
\hline 3 & $\mathrm{M}$ & $\mathrm{P}$ & $19-36(\mathrm{~K}-\mathrm{Kr})$ & 18 \\
\hline 3 & $\mathrm{M}(\mathrm{P})$ & $\mathrm{M}$ & $37-54(\mathrm{Rb}-\mathrm{Xe})$ & 18 \\
\hline 4 & $\mathrm{~N}$ & $\mathrm{O}$ & $55-86(\mathrm{Cs}-\mathbf{R n})$ & 32 \\
\hline 4 & $\mathrm{~N}(\mathrm{O})$ & $\mathrm{N}$ & $87-118(\mathbf{F r}-\mathbf{O g})$ & 32 \\
\hline
\end{tabular}

Table 1: Grouping of elements

Grouping is shown in Table 1. There are two possibilities - either the shells $\mathbf{L}, \mathbf{M}$ and $\mathbf{N}$ are doubled or the grouping is reflected after the $\mathbf{N}$ shell, so shells $\mathbf{O}, \mathbf{P}$ and $\mathbf{Q}$ contain the same number of elements such as shells $\mathbf{N}, \mathbf{M}$ and $\mathbf{L}$, respectively. Note that in case of alternative $(\mathbf{O g})$ grouping, no elements beyond $\mathbf{O g}$ are theoretically possible - otherwise another shell would be present between $\mathrm{He}$ and $\mathbf{L i}$. 
Gravitational constant $\mathbf{G}$ is not dimensionless and therefor not invariant to vertical scale transformation.

On the standard atom scale $\mathbf{U}_{\mathbf{0}}$, gravitational constant for a completely neutralized general force can be derived from previously obtained orbital momentum of the Carbon-10 outermost electron:

$$
\begin{gathered}
\frac{m v^{2}}{r}=G \frac{M m}{r^{2}} \\
v^{2}=G \frac{M}{r} \\
M=\frac{\text { Sun mass }}{\text { Neptune mass }} * m \\
G=G_{0}=v^{2} \frac{r}{M}=1.234879253 * 10^{27} \frac{m^{3}}{k g s^{2}} \\
v=v_{\mathrm{U}_{0}}=5.585837356 * 10^{5} \mathrm{~m} / \mathrm{s} \\
r=\mathrm{U}_{0}=70 * 10^{-12} \mathrm{~m}
\end{gathered}
$$

where $\mathbf{m}, \mathbf{v}, \mathbf{r}$ are components of the outermost electron orbital momentum (mass, velocity, radius).

If one now, equalizes electric with gravitational force (for photon/graviton $\mathrm{m}>\mathbf{0}-$ Yukawa, Proca ${ }^{71}$ ):

$$
k_{0} Q^{2}\left(\frac{1}{r^{2}}+\frac{\mu_{\gamma}}{r}\right) e^{\left(-\mu_{\gamma} r\right)}=G_{0} m^{2}\left(\frac{1}{r^{2}}+\frac{\mu_{n}}{r}\right) e^{\left(-\mu_{n} r\right)}
$$

discarding $\boldsymbol{\mu} / \mathbf{r}$ factors due to being practically equal and equal to $\mathbf{0}$ on both sides (expecting large $\mathbf{r}$ ):

$$
\begin{gathered}
\mu_{n}-\mu_{\gamma}=\frac{1}{r} \ln \left(\frac{G_{0} m^{2}}{k_{0} Q^{2}}\right) \\
\frac{M_{n} c_{n}}{\hbar_{n}}-\frac{M_{\gamma} c_{\gamma}}{\hbar_{\gamma}}=\frac{1}{r} \ln \left(\frac{G_{0} m}{k_{0} Q}\right) \\
\frac{c_{n}}{\hbar_{n}}=\frac{c_{\gamma}}{\hbar_{\gamma}}=\frac{c}{\hbar} \\
M_{n}-M_{\gamma}=\frac{\hbar}{c} \frac{1}{r} \ln \left(\frac{G_{0} m^{2}}{k_{0} Q^{2}}\right)
\end{gathered}
$$




$$
\begin{array}{r}
c=2.99792458 * 10^{8} \mathrm{~m} / \mathrm{s} \\
\mathrm{k}_{0}=\text { Coulomb constant }=8.9875517873681764 * 10^{9} \mathrm{Nm}^{2} / \mathrm{C}^{2} \\
Q=\text { electron charge }=1.60217733 * 10^{-19} \mathrm{C} \\
M_{Y}=\text { photon mass } \\
M_{n}=U_{0} \text { graviton mass }
\end{array}
$$

Using previously obtained photon mass $M_{Y}=2 * 9.10938356 * 10^{-73} \mathrm{~kg}$ and carbon graviton neutrino mass $M_{n}=2 * 1.663337576 * 10^{-68} \mathrm{~kg}$, this gives:

$$
r=1.3032821975 * 10^{26} \mathrm{~m}
$$

as the distance in space when two forces become equal.

Note that, with current arrangement, obtained $\mathbf{r}$ is actually negative. Positive $\mathbf{r}$ is obtained if $\mathbf{M}_{\mathrm{Y}}$ and $\mathbf{M}_{\mathrm{n}}$ exchange masses.

Graviton neutrino is bosonic (half-neutrino/anti half-neutrino pair = e-neutrino/anti e-neutrino pair) differing from photon only in charge/mass ratio, so the exchange is not impossible, rather expectable.

As shown previously, components of general force, charge and mass are exchangeable through inflation/deflation of momentum components (even in neutral particles, the amount of gravitational mass can increase at the expense of charge mass, with particle remaining neutral).

Nature of the force thus has to oscillate over distance.

Taking into account error margins, obtained distance is equal to the radius of observable universe, assuming currently accepted [img] age $\left(13.799 * 10^{9}\right.$ years $)$, constant speed of light and flat space:

$$
r=c \Delta t=2.99792458 * 10^{8} * 13.799 * 10^{9} * 365.25 * 24 * 60 * 60=1.305 * 10^{26} \mathrm{~m}
$$

The fact that obtained distance is equal to the radius of observable universe is not a coincidence.

For an inflation at the speed of light, distance in space is distance in time $\left[{ }^{*} \mathbf{c}\right]$ so this may be interpreted as the time when both forces (carrier masses) were equal, after which point one particle started loosing mass while the other was gaining mass.

Note that previously obtained real age of observable universe $\left(12.75 * 10^{9}\right.$ years $)$ implies inflation was at times faster than current $\mathbf{c}$ which, for the same radius, implies the $\mathbf{c}$ in flat space was also higher at these 


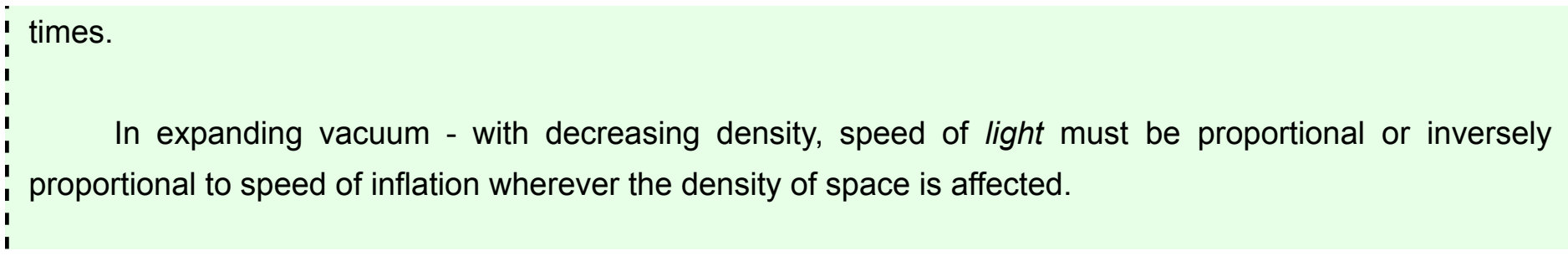

In the past the observable universe did expand, but geometry deformation was localized (quantized, gravitational wells being the quanta of vacuum) and expansion may have lasted only up to the point of CMB emission (at this point the speed of light also became equal to $\mathbf{c}$ ). The redshifts thus may be caused by lower scale $\left(\mathbf{U}_{-2}\right)$ particles in intergalactic medium absorbing photon energies.

Taking into account the scaled density of the observable universe (gas), evidently this is a discontinuity, a gravitational maximum between layers of, relatively, dense matter.

Since maximum speed in such discontinuities is Keplerian velocity (angular velocity of space) of the maximum, in this case equal to $\mathbf{c}$, this is a black hole maximum (escape velocity $=\sqrt{ } \mathbf{2} \mathbf{c}$ ).

Thus, light coming from large distances might be the light reflected off of the firewall, providing a window to the past of inner content. This explains the correlation of apparently spatially separated phenomena (galaxies) - these may not be images of different phenomena separated in space, but one separated in time (note that the decay, inverse decay of a graviton neutrino and photon can also explain the effect - neutrino and photon emitted from the same source may produce images of different luminosity if a neutrino decays to photon).

Note that, if one fixes the gravitational constant $\mathbf{G}_{0}$ to

$$
G_{0}=1.257920328 * 10^{27} \frac{\mathrm{m}^{3}}{\mathrm{kgs}^{2}}
$$

one obtains this:

$$
\begin{gathered}
\frac{G_{0} m}{k_{0} Q}=K^{-1} \mu_{0}{ }^{-1}=\mu_{0}^{-1} \\
c^{2}=4 \pi \frac{G_{0} m}{Q} K=4 \pi \frac{G_{0} m}{Q}
\end{gathered}
$$

where $\mu_{0}$ is the vacuum permeability (magnetic) constant and $K=1 \mathrm{C} / \mathrm{m}$.

One can now obtain $\mathbf{k}$ and $\mathbf{Q}$ for the $\mathbf{U}_{\mathbf{1}}$ scale (Solar System): 


$$
\begin{gathered}
\frac{k_{1} Q_{1}^{2}}{G_{1} m_{1}^{2}}=\frac{k_{0} Q_{0}{ }^{2}}{G_{0} m_{0}^{2}} \\
k_{1}=\frac{k_{0} Q_{0}^{2}}{G_{0} m_{0}^{2}} G_{1} \frac{c_{1}^{4}}{16 \pi^{2} G_{1}^{2}} K_{1}^{-2}=\frac{k_{0} Q_{0}{ }^{2}}{G_{0} m_{0}{ }^{2}} \frac{c_{1}{ }^{2}}{16 \pi^{2} G_{1}}
\end{gathered}
$$

Using $G_{1}=6.674 * 10^{-11} \mathrm{~m}^{3} / \mathrm{kgs}^{2}$ and previously obtained $c_{1}=2.930445979 * 10^{6} \mathrm{~m} / \mathrm{s}$ :

$$
\begin{gathered}
k_{1}=3.95052951 * 10^{38} \frac{\mathrm{Nm}^{2}}{\mathrm{C}^{2}} \\
Q_{1}=10001.92779151 C \approx 1 * 10^{4} \mathrm{C}
\end{gathered}
$$

Ranges on $U_{1}$ scale:

$$
\begin{aligned}
& M_{\gamma_{1}}-M_{n 1}=\frac{\hbar_{1}}{c_{1}} \frac{1}{r} \ln \left(\frac{G_{1} m_{1}}{k_{1} Q_{1}}\right) \\
& \hbar_{1}=\frac{h_{m 2}}{2 \pi}=7.95683841 * 10^{40} \mathrm{Js}
\end{aligned}
$$

Using $m_{1}=1.02413 * 10^{26} \mathrm{~kg}$ and previously obtained $M_{\mathrm{n}_{1}}=1.663337576 * 10^{-26} \mathrm{~kg}, \mathrm{M}_{\mathrm{V}_{1}}=9.10938356$ ${ }^{*} 10^{-31} \mathrm{~kg}$, the distance where two forces become equal, $\mathrm{r}=1.0059686 * 10^{62} \mathrm{~m} \approx 1 * 10^{62} \mathrm{~m}$.

Note that, if one fixes $\mathbf{m}_{\mathbf{1}}$ to

$$
m_{1}=0.99026311 * 10^{26} \mathrm{~kg} \approx 1 * 10^{26} \mathrm{~kg}
$$

one obtains this:

$$
\frac{G_{1} m_{1}}{k_{1} Q_{1}}=K_{2} M_{p}=M_{p}
$$

where $M_{p}=1.6726218977 * 10^{-27} \mathrm{~kg}$ is the mass of a standard proton.

Range of $U_{1}$ electric force:

$$
\lambda_{\gamma_{1}}=\frac{\hbar_{1}}{c_{1}} \frac{1}{M_{\gamma_{1}}}=2.98069699 * 10^{64} \mathrm{~m} \approx 3 * 10^{64} \mathrm{~m}
$$

Range of $U_{1}$ gravitational force:

$$
\lambda_{n 1}=\frac{\hbar_{1}}{c_{1}} \frac{1}{M_{n 1}}=\frac{\hbar_{1}}{5 * 10^{-20}}=1.63239937 * 10^{60} \mathrm{~m}
$$


Here, unit $\mathbf{m}$ (meter) is unscaled, for a properly scaled metric the ranges are equal to ranges on $\mathbf{U}_{\mathbf{0}}$ scale. 


\section{Relation of G variation to Sun's discontinuities}

Equalizing the strength of electric and gravitational force between two free particles (positron and electron), disregarding small mass of carrier particles:

$$
\begin{aligned}
& k_{0} \frac{Q^{2}}{r^{2}}=G \frac{M^{2}}{r^{2}} \\
& \frac{1}{4 \pi \varepsilon_{0}} Q^{2}=G M^{2}
\end{aligned}
$$

yields the following value for the gravitational constant $\mathrm{G}$ :

$$
\begin{aligned}
& G=\frac{k_{0} Q^{2}}{M^{2}}=2.78025476 * 10^{32} \frac{\mathrm{m}^{3}}{k g s^{2}} \\
& \mathrm{k}_{0}=8.9875517873681764 * 10^{9} \mathrm{Nm}^{2} / \mathrm{C}^{2} \\
& Q=1.60217733 * 10^{-19} \mathrm{C} \\
& M=9.10938356 * 10^{-31} \mathrm{~kg}
\end{aligned}
$$

In $\mathbf{C R}$, gravitational constant $\mathbf{G}$ changes with scale. But it is also modified with neutralization of EM force, when $\mathbf{k}_{\mathbf{0}}$ decreases, while $\mathbf{G}$ increases.

This enables the gravitational force to be, at least in some cases, a prevailing force in the atom, rather than EM force.

I have previously calculated $\mathbf{G}$ relative to a ${ }^{10} \mathrm{C}$ atom nucleus mass obtained through current Sun mass, the constant $\mathbf{G}$ using rest mass of ${ }^{10} \mathrm{C}$ nucleus is:

$$
G_{0}=v^{2} \frac{r}{M}=1.29864745 * 10^{27} \frac{\mathrm{m}^{3}}{k g s^{2}}
$$

$$
\begin{array}{r}
v=5.5550351679 * 10^{5} \mathrm{~m} / \mathrm{s} \\
r=70 * 10^{-12} \mathrm{~m} \\
M=1.663337576 * 10^{-26} \mathrm{~kg}
\end{array}
$$

where $\mathbf{m}, \mathbf{v}$ and $\mathbf{r}$ are components of the orbital angular momentum of the outermost electron.

Calculated $\mathbf{G}\left(\mathbf{G}_{\mathbf{0}}\right)$ is now only $\mathbf{5}$ orders of magnitude smaller than $\mathbf{G}$ required for gravity to be equal in strength to EM force between an electron and a positron. 
But instead of $\mathbf{G}$ increasing, one might assume that $\mathbf{k}_{\mathbf{0}}$ decreases by $\mathbf{5}$ orders of magnitude, or more precisely by this amount:

$$
\Delta k=\frac{G}{G_{0}}=2.140884935 * 10^{5}
$$

Thus, the increase of $\mathbf{G}(\boldsymbol{\Delta} \mathbf{G})$ of Earth's inner core maximum, after extraction, neutralization and collapse to current radii, is equal to $\Delta \mathbf{k}$.

I have previously calculated that this $\mathbf{G}$ has increased to $5.731534632 * 10^{-6} \mathrm{~m}^{3} / \mathrm{kgs}^{2}$, which is, relative to surface $\mathbf{G}\left(6.674 * 10^{-11} \mathrm{~m}^{3} / \mathrm{kgs}^{2}\right)$, an increase of:

$$
\Delta G=\frac{5.731534632 * 10^{-6}}{6.674 * 10^{-11}}=8.58785531 * 10^{4}
$$

which is also the ratio between imaginary mass $\mathbf{M}\left(5.97 * 10^{24} \mathrm{~kg}\right)$ and real mass $\mathrm{m}\left(6.95 * 10^{19} \mathrm{~kg}\right)$ of Earth.

Gravitational constant $\mathbf{G}$ measured on the surface of the Earth (surface $\mathrm{G}$ ) is relative to standard scale $\left(\mathbf{U}_{\mathbf{0}}\right)$, a proper $\mathbf{G}$ for $\mathbf{U}_{\mathbf{1}}$ scale must be different.

But what was the initial G of Earth's inner core?

According to above hypothesis, it should have been:

$$
G_{i}=\frac{5.731534632 * 10^{-6}}{\Delta k}=2.677180141 * 10^{-11} \frac{\mathrm{m}^{3}}{\mathrm{kgs}^{2}}
$$

If Earth's core has been extracted from the Sun, as hypothesized, one can get it's original radius using this constant:

$$
\begin{aligned}
& r=\sqrt{\frac{G_{i} M}{g}}=440784499.323 \mathrm{~m} \approx 440785 \mathrm{~km} \\
& M=\text { img mass of the Sun }=1.988500 * 10^{30} \mathrm{~kg} \\
& \mathrm{~g}=\text { gravity of the maximum }=274 \mathrm{~m} / \mathrm{s}^{2}
\end{aligned}
$$

This agrees very well with the hypothesis of entanglement of discontinuities with inner planetary orbitals:

$$
\frac{r}{R} \approx \frac{r_{E}}{r_{M}} \approx \frac{2}{3}
$$




$$
\begin{array}{r}
R=\text { Sun radius }=695700 \mathrm{~km} \\
r_{E}=\text { Earth orbital }=149.6 * 10^{6} \mathrm{~km} \\
r_{M}=\text { Mars orbital }=227.92 * 10^{6} \mathrm{~km}
\end{array}
$$

The discontinuity $(r / R=\mathbf{0 . 6 3})$ is evident through the profile of rotational velocities of the Sun:

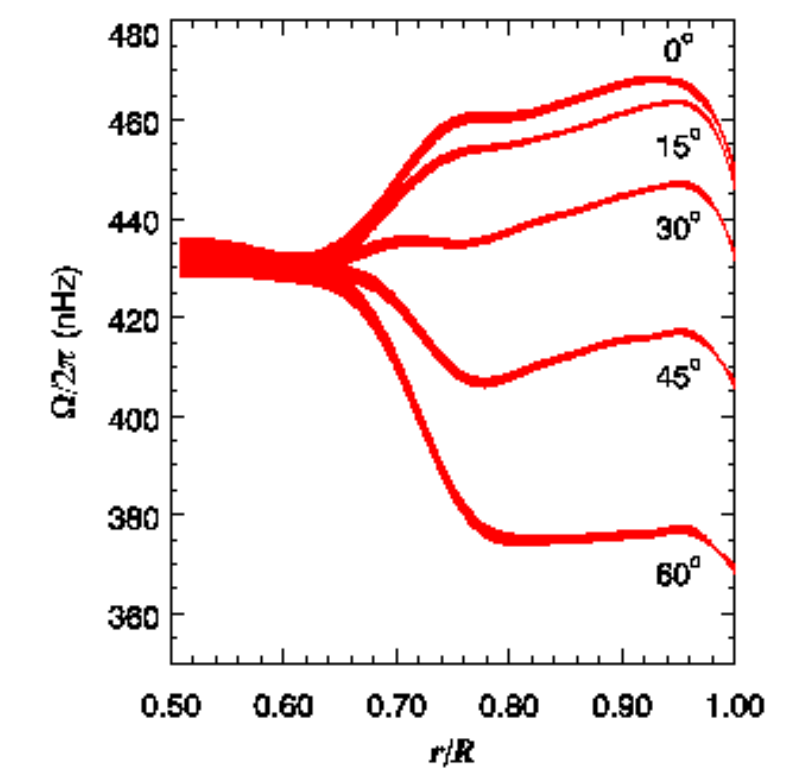

NSO/NSF

Fig. 1: Sun rotation rates ${ }^{72}$

Above this discontinuity is the tachocline (transition region between the radiative and convective layer of the Sun), a major source of the Sun's magnetic dipole, analogous to the region of charge above Earth's inner core.

The hypothesis of neurogenesis, assuming pending neurogenesis on Earth and completed neurogenesis on Mars and other terrestrial planets, explains why Earth is the only one with an active surface magnetic dipole. The connection of tachocline with $\mathbf{0 . 6 3 R}$ discontinuity would suggest:

1. it's position is not permanent and it moves between discontinuities, corresponding to the planet with ongoing neurogenesis,

2. possible multiple active discontinuities and associated tachoclines in the past, initially at maximum, or

3. current position is the place of birth of all planetary embryos (cores).

The 2 nd hypothesis here is most plausible - the tachocline is active as long as the magnetic dipole of the corresponding planet is active (the two phenomena are synchronized).

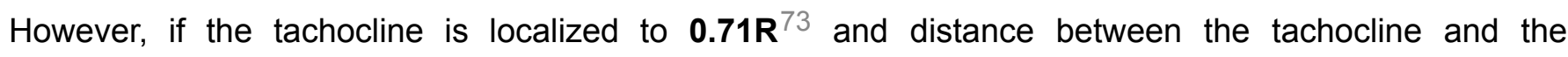
discontinuity is scaled from Earth (distance between the charge radius and gravitational maximum), the associated discontinuity is at: 


$$
r=\frac{1206115}{1705704} 0.71 R=0.5 R \approx \frac{1}{\sqrt{2}} 0.71 R
$$

which would be a discontinuity associated with Venus.

In that case the tachocline is the location of a charge radius associated with a $\mathbf{0 . 5 R}$ gravitational maximum and, assuming equal g-factor, such charge radius should also be located at:

$$
r=\frac{1705704}{1206115} R=983868.265 \mathrm{~km} \approx \sqrt{2} R
$$

In this case though, the g-factor of a neutron might be more appropriate, yielding $r=1.111507303 * 10^{6}$ $\mathrm{km}$ (and a mirror at $444533.257 \mathrm{~km}=\mathbf{0 . 6 3 9 R}$ ).

Note that the $\mathbf{0 . 7 1 R}$ tachocline is $3 / 4$ of $\mathbf{0 . 9 4 R}$, which according to Fig. 1 seems to be another discontinuity or a fossilized initial Sun radius.

Such fossil is also visible at $\mathbf{0 . 7 5 R}$, which should be a discontinuity in $4 \mathrm{p} 6 \mathrm{n}$ state.

The $\mathbf{0 . 6 3 R}(\mathbf{2} / 3$ of $\mathbf{0 . 9 4 R})$ is also a fossil, as the current location associated with Earth is $\mathbf{0 . 6 6 R}$.

Note that $0.63 R$ discontinuity is, similarly to $0.4 R(2 / 5 R)$ discontinuity, weak (unstable) - it may not always be present in the rotational profile of the Sun.

The $\mathbf{0 . 6 3 R}$ has been revealed in seismic analysis (periodic, $1.3 \mathrm{y}$ signal), and possibly the $\mathbf{0 . 4 R}$ discontinuity too (noted as a low significance bump in rotation variability between $\mathbf{0 . 2 R}$ and $\mathbf{0 . 6 R}$ ) $^{74}$.

Sun's $\mathbf{G M}$ product has increased $\mathbf{0 . 0 6 \%}$ due to kinetic energy relative to $\mathbf{C M B}$, so initial radius at $\mathbf{0 . 9 4 R}$ implies that surface radius changes proportionally:

$$
R=\frac{R_{0}}{\sqrt{1-\frac{v^{2}}{c_{1}^{2}}}}
$$

for previously obtained $c_{1}=2.93 * 10^{6} \mathrm{~m} / \mathrm{s}$ and $\mathbf{v}=v_{\mathbf{s}}+v_{p}=996 \mathrm{~km} / \mathrm{s}$, gives $R_{0}=654271.142 \mathrm{~km}=0.94 R$.

\section{Note 1:}

This is analogous to the decrease of Bohr radius due to relativistic mass of the electron. Bohr radius: 


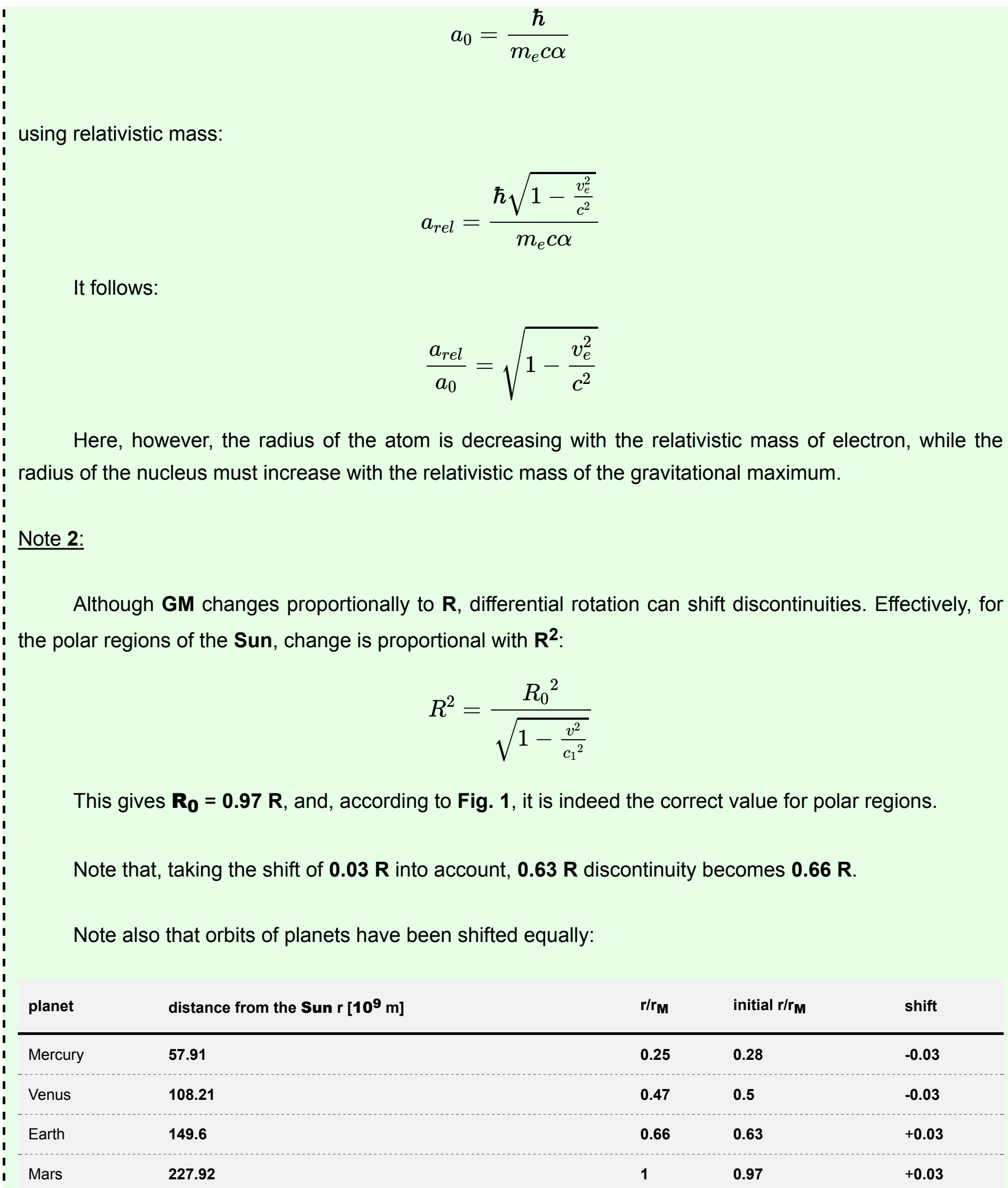

The Earth has thus moved from $0.63 r_{\mathbf{M}}$ to $0.66 \mathbf{r}_{\mathbf{M}}$, while Venus moved equally but in opposite direction, from 0.5 to 0.47 . Mars moved from 0.97 to $1 r_{M}$ and Mercury too moved accordingly. 
Calculated initial radii, location of discontinuities and inner planets together with fossil evidence show the following:

- radii of gravitational maximums in the system change physically and proportionally to the change in momentum of the system,

- relativistic effects of time dilation and length contraction are a direct consequence of physical changes of space in the system (atom),

- since used velocities are relative to $\mathbf{C M B}$ the radii must have been fossilized at the time of CMB emission.

Kinetic energy changes the system on all scales, proportionally to the scale of a gravitational maximum. This constant of proportionality (or unit quanta) is the speed of light $\mathbf{c}$, which at the Sun scale $\left(\mathbf{U}_{\mathbf{1}}\right)$ is equal to $\mathbf{c}_{\mathbf{1}}=$ $2.93 * 10^{6} \mathrm{~m} / \mathrm{s}$, while on the scale of the standard ${ }^{10} \mathrm{C}$ atom nucleus $\left(U_{0}\right)$ is equal to $c_{0}=2.99792458 * 10^{8}$ $\mathrm{m} / \mathrm{s}$.

Note that all these constants are relative to a local universe characterized by the CMB (Constant Microwave Background) radiation.

CMB is thus not part of some special absolute space:

- if increase in kinetic energy increases the energy of the system and units of measurements (quanta of observational energy) then the initial state does not exist - what one considers the initial state of the system (and the current state of CMB) is the result of kinetic energy of the space associated with CMB the Solar System can thus be the actual standard carbon atom inflated due to this kinetic energy,

- but there is no reason for the carbon atom or any other particle to be something elementary, this goes to infinity.

The CMB space is thus only relatively special and it is so only due to limits in our senses (observational power) - we do not have the ability to detect radiation of the smaller scale than $U_{-1}$ photon, nor we have the ability to see how fast and relative to what the observable universe moves (we can only be pretty confident it rotates around something).

Our senses are effectively limited to the scale of the standard atom $\mathbf{U}_{\mathbf{0}}$ (we are the composition of atoms of this scale) and adjacent vertical scales $U_{1}$ and $U_{-1}$. 


\section{Gyro-magnetic ratio and its correlation with Earth/Moon}

The gyro-magnetic ratio of a particle is the ratio of its magnetic moment to its angular momentum:

$$
\gamma=\frac{\mu}{L}
$$

With the assumption that mass and charge have equal momentum:

$$
\gamma=\frac{\mu}{L}=\frac{q}{2 m}
$$

where $\mathbf{q}, \mathbf{m}$ are charge and mass of the particle, respectively.

Measurements show that this is not valid for quantum particles such as electron. Thus, a dimensionless factor $g_{\mathbf{e}}$ (g-factor) was introduced:

$$
\gamma=\frac{q}{2 m} g_{e}
$$

The factor has been attributed to quantum effects which do not exist in classical (intuitive) reality - point particles with intrinsic magnetic moment (no rotation).

The notion of point particles having any properties is in itself problematic, let alone existence of different point particles with different properties. However, if such particles could exist, due to scale invariance, they would have to exist on bigger scales too. No such thing has ever been observed in reality - all magnetic fields are produced by moving charges of objects having a real radius.

Thus, intrinsic magnetic momentum is not intuitive, but intrinsic rotation of charge (producing the momentum) at finite radius greater than $\mathbf{0}$ is.

In CR there is also no intrinsic coupling of matter and gravity, and since charge field is a polarized gravitational field, the $\mathbf{g - f a c t o r}$ can be explained simply by a difference in distribution (or angular momentums) of gravitational mass and charge mass within the particle, preserving the intuitive concepts of reality.

Complete relativity not only allows speeds faster than light (photon mass is scale dependent) but implies such speeds must exist at some scale, thus the required superluminal rotation of charge (implied at certain radii) in particles such as an electron is not an issue either.

The absolute (invariant) speed limit is not a dimensionless constant and thus is counter-intuitive in scale invariant reality (relativity), but, in this case, the required speed would be valid even in the context of General Relativity (charge is at rest relative to rotating space) if it would incorporate scale invariant curvature of space. 
Magnetic moment $\boldsymbol{\mu}$ and angular momentum L:

$$
\begin{gathered}
\mu=I A=\frac{q v_{c}}{2 \pi r_{c}} \times \pi r_{c}{ }^{2} \\
L=m v_{m} \times r_{m}
\end{gathered}
$$

where $\mathbf{v}_{\mathbf{c}}, \mathbf{r}_{\mathbf{c}}$ are the charge orbital velocity and radius, respectively, and $\mathbf{v}_{\mathbf{m}}, \mathbf{r}_{\mathbf{m}}$ are the mass orbital velocity and radius, respectively.

The factor $\mathbf{g}_{\mathbf{e}}$ is thus:

$$
g_{e}=\frac{v_{c} \times r_{c}}{v_{m} \times r_{m}}
$$

Being dimensionless, it should be scale invariant relative to particle flavor.

This means that the value of $\mathbf{g}_{\mathbf{e}}$ for electron and positron is equal to $\mathbf{g}_{\mathbf{e}}$ of Earth, as Earth is a large scale Dirac fermion equivalent (obviously not a point particle unless taken relatively).

Just like the electron, the Earth consists of intrinsic charge and mass and accumulated mass due to neutralization.

The intrinsic energy is contained within the inner and outer core.

Assuming charge radius is in the outer core where gravity equals $g_{c}=137 \mathrm{~m} / \mathrm{s}^{2}$ and gravitational mass radius is the inner core gravitational maximum $g_{m}\left(274 \mathrm{~m} / \mathrm{s}^{2}\right)$, with equal rotation period (and angle between $v$ and $r$ vectors):

$$
g_{e}=\frac{v_{c} \times r_{c}}{v_{m} \times r_{m}}=\frac{r_{c}^{2}}{r_{m}^{2}}=\frac{g_{m}}{g_{c}}=2
$$

Note that it was assumed that mass is not a solid body with radius $\mathbf{r}_{\mathbf{m}}$ but, like the charge, a particle or a stream of particles forming a ring at $\mathbf{r}_{\mathbf{m}}$.

This is a valid assumption since this mass is not real mass, but vacuum energy (imaginary mass) which, in case of charged naked maximums, forms a ring rather than sphere surface. 


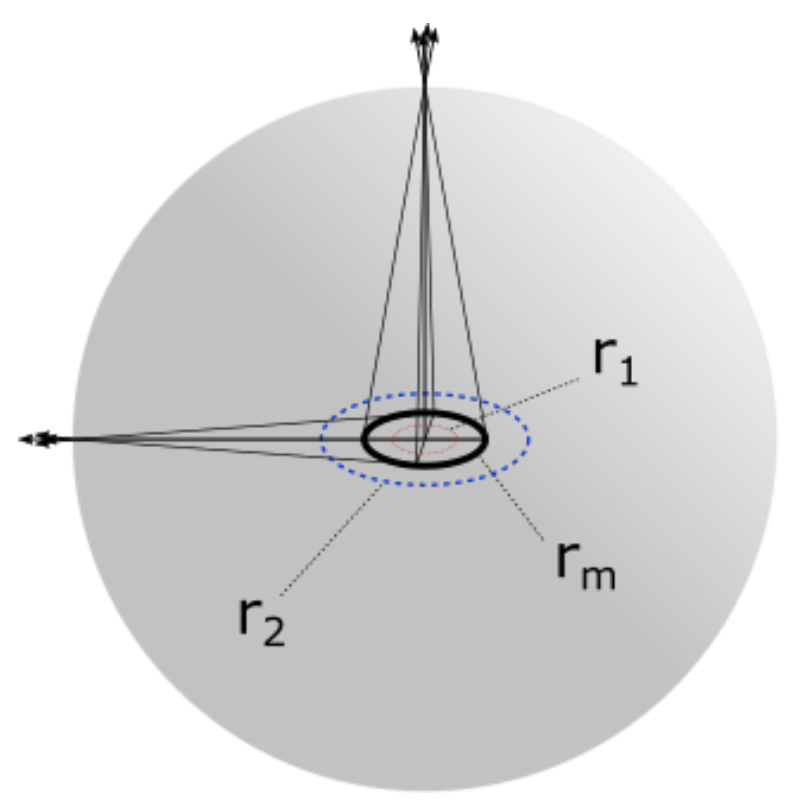

Fig. 1: Mass and charge radius of charged bodies

Since gravitational potential is not isotropic, gravitational acceleration at any point is a vector sum of accelerations induced by vacuum quanta forming the ring:

$$
g=\sum_{k=1}^{n} \vec{g}_{k}=\sum \frac{G_{0} M_{0}}{\overrightarrow{d r}^{2}}
$$

In case of equatorial and polar gravity vector components parallel to surface cancel out.

Equatorial gravity is thus:

$$
g=\sum_{k=1}^{n} \frac{G_{0} M_{0}}{\left[R_{e}-r_{m} \cos \left(k \frac{2 \pi}{n}\right)\right]^{2}}
$$

where $\mathbf{R}_{\mathrm{e}}$ is the equatorial radius.

Polar gravity:

$$
g=\sum_{k=1}^{n} \frac{G_{0} M_{0}}{R_{p}{ }^{2}}=n \frac{G_{0} M_{0}}{R_{p}{ }^{2}}
$$

where $\mathbf{R}_{\mathrm{p}}$ is the polar radius.

Deriving $\mathbf{G}_{\mathbf{0}} \mathbf{M}_{\mathbf{0}}$ product with equatorial gravity fixed to $9.798 \mathrm{~m} / \mathrm{s}^{2}$ and calculating polar gravity, for $\mathbf{n}>=\mathbf{5}$, gives $9.34 \mathrm{~m} / \mathrm{s}^{2}$. 
This is smaller than measured, so the Earth must be a composite of $\mathbf{2}$ positrons (or positron equivalents), as hypothesized.

Note that I have previously hypothesized that the shape of a gravitational maximum with charge neutralization is transforming from a ring like to sphere surface form.

Here, it is assumed that ring form is preserved, for the sake of proving fossilization of initial conditions.

It is also assumed that gravity is provided by the gravitational maximums, rather than real mass [shielding the gravity of the maximums], however, effectively there is no difference for surface gravity (real mass imitates the maximum).

With 2 particles in the same state, energy splits into two levels:

a)

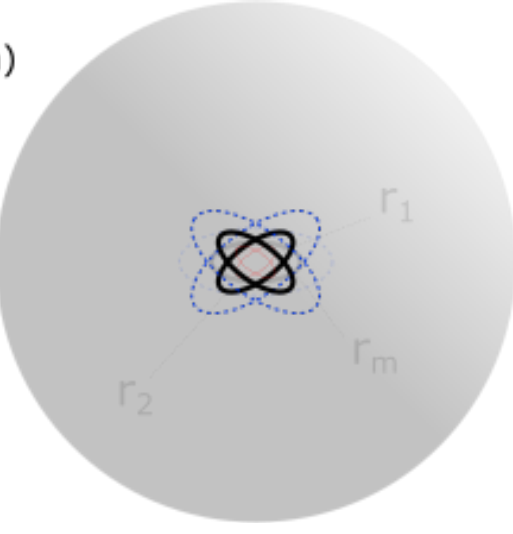

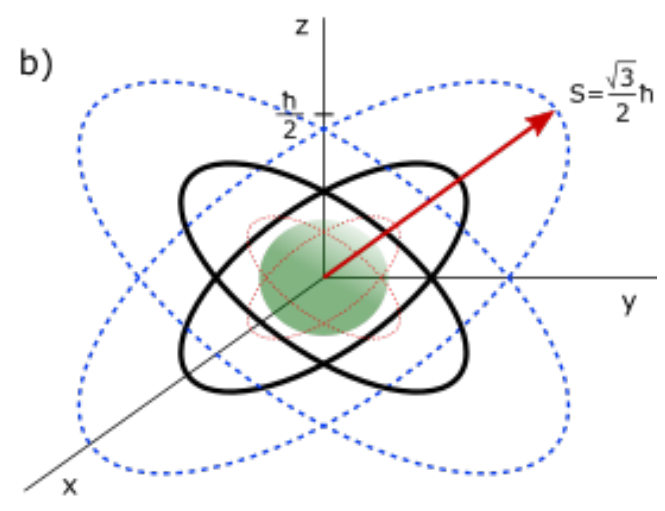

Fig. 2: Mass and charge radius of two charged bodies sharing a single state

In such state, two charges are deflected from the equator by this angle:

$$
\Delta \varphi=\sin ^{-1} \frac{1}{\sqrt{3}}=35.2643896827547^{\circ}
$$

Charges are thus separated by $2^{\star} 35.2643896827547^{\circ}=70.5287793655094^{\circ}$ (two magnetic north poles on Jupiter are separated by this angle, confirming it's 2 e configuration).

Confirmation of this configuration of Earth comes from the state of the Moon (Luna) and non-alignment of Earth's north and south magnetic poles.

Total obliquity of Luna relative to Earth's equator is $23.44^{\circ}+5.14^{\circ}+6.68^{\circ}=35.26^{\circ}$, equal to $\Delta \varphi$.

The Moon orbits one of Earth's positrons and its obliquity shows that it is built around one of the collapsed gravitational maximums of this positron. 
One can thus expect this positron to have smaller contribution to gravity and charge of Earth. Further splitting of energy levels due to carbon configuration can also be expected, so number of quanta should be $\mathbf{6}$ in one positron and 5 in the other ( 1 is in the Moon).

It appears that, in the collapse, $6.68^{\circ}$ of Luna's obliquity to Earth's equator has been exchanged for obliquity to Luna's own equator, this can be due to influence from another body, but, since the loss of one quantum causes asymmetry in charge distribution it is more likely that this is the exact amount by which the inner positron decreased its angle to Earth's equator.

Thus, one can expect the orbital plane of this positron to be aligned with the orbital plane of the Moon.

This can then be interpreted as redistribution of charges on the plane, rather than loss. The Moon is thus the reason why Earth still has a dipole magnetic field - with symmetric anti-aligned positron spins the magnetic dipole would be canceled.

Bigger moons and/or an increased number of moons (with distinct gravitational wells) of outer planets with stronger magnetic fields are thus no surprise and indicate core asymmetry if the spins are anti-aligned (note that a symmetric core does not indicate a planet has no moons, rather that it has the same number of them on each orbital plane).

But rather than the extraction of the Moon core from Earth, in the current, progressive evolution a reversed scenario is more plausible.

Even if the first positron was not fragmented from the beginning, massive extinctions that happened on Earth suggest the second one arrived quantum by quantum on a periodic basis.

There were $\mathbf{5}$ massive extinctions and there are $\mathbf{5}$ quanta of the positron in the core, $\mathbf{1}$ in the Moon.

As the mammal brain has 6 layers, with 6th layer sparsely populated, the theory of neurogenesis is strongly aligned with this hypothesis. Note that the sparse neuron cell population of the 6th layer now indicates an underdeveloped layer - the direct cause for this is the distance of the Moon.

Since this distance is variable it explains the variation in intelligence among individuals. A Moon in perigee at the point of formation of the 6th brain layer would increase general intelligence (at the time of formation of other layers would probably impact other skills).

This is not a big increase, but enough to create a difference and allow weak evolution of intelligence, as brain structure is a genetic factor. 
Current increasing Moon distance and the fact that our brain size started decreasing 10-15k years ago support the hypothesis of such entanglement.

As the Moon fuses with Earth, one can thus expect a strong evolution of the 6th layer in brains of species (including the brain of Earth itself).

One must now ask whether the position of other planets and the Sun impact the development? Most likely, but not as much.

Interesting is the fact that one has $\mathbf{5}$ vital organs - these are thus likely entangled with other $\mathbf{5}$ quanta of the positron associated with the Moon, so variation in the state of these can be determined by organic variation between individuals. Strong disturbance could thus cause mutation in evolution.

Thus, one can not only expect our 6th brain layer to expand during the next strong evolution event, but also a new vital organ (a 6th sense) and mutation of body into new species. 


\section{References}

1. ${ }^{1}$ Complete Relativity: Nature of observables (2021), Amenoum https://amenoum.org/complete_relativity.html

2. NASA Planetary Fact Sheet ${ }^{2}$

https://nssdc.gsfc.nasa.gov/planetary/factsheet/

3. ${ }^{3}$ Images of planets (2013), OpenClipart-Vectors https://pixabay.com/users/openclipart-vectors-30363/

4. ${ }^{4}$ Images of planets (2013), OpenClipart-Vectors https://pixabay.com/users/openclipart-vectors-30363/

5. ${ }^{5}$ Six transiting planets and a chain of Laplace resonances in TOI-178 (2020), A. Leleu et al https://doi.org/10.1051/0004-6361/202039767

6. ${ }^{6}$ CODATA recommended values (2018), NIST https://physics.nist.gov/cuu/Constants/bibliography.html

7. ${ }^{7}$ Six transiting planets and a chain of Laplace resonances in TOI-178 (2021), A. Leleu et al https://doi.org/10.1051/0004-6361/202039767

8. ${ }^{8}$ An Electroweak model with electrons of opposite helicities carrying the same quantum numbers (1981), W. B. Yeung

9. ${ }^{9}$ Asymptotic g modes: Evidence for a rapid rotation of the solar core (2017), Fossat et al https://doi.org/10.1051/0004-6361/201730460

10. ${ }^{10}$ The Relationship of Sunspot Cycles to Gravitational Stresses on the Sun: Results of a Proof-ofConcept Simulation (2011), D. Jassby https://doi.org/0.1016/B978-0-12-385956-3.10014-2

11. ${ }^{11}$ Internal rotation of Sun (2010), SOHO https://ase.tufts.edu/cosmos/view_picture.asp?id=1368

12. ${ }^{12}$ Velocity Curves for Spiral Galaxies (2015), C. R. Nave 
http://hyperphysics.phy-astr.gsu.edu/hbase/Astro/velcurv.html

13. ${ }^{13}$ Discovery of variation in Solar coronal rotation with altitude (2001), H. O. Vats et al

14. ${ }^{14}$ The Titius-Bode law and a quantum-like description of the planetary systems (2005), F. Scardigli

15. ${ }^{15}$ Self-Organizing Systems in Planetary Physics: Harmonic Resonances of Planet and Moon Orbits (2017), M. J. Aschwanden

16. ${ }^{16}$ Eccentricity of the geomagnetic dipole caused by lopsided inner core growth (2012), P. Olson et al https://doi.org/10.1038/NGEO1506

17. ${ }^{17}$ Search for new phenomena in dijet events with the ATLAS detector at $\sqrt{ } \mathbf{s}=13 \mathrm{TeV}$ (2019), D. Zhang https://cds.cern.ch/record/2683921/files/ATL-PHYS-SLIDE-2019-426.pdf

18. ${ }^{18}$ Signals of a 2 TeV W' boson and a heavier Z' boson (2016), B. A. Dobrescu et al https://doi.org/10.1007/JHEP05(2016)047

19. ${ }^{19}$ Measurements of Newton's gravitational constant and the length of day (2015), J. D. Anderson et al https://doi.org/10.1209/0295-5075/110/10002

20. ${ }^{20}$ Horizons On-Line Ephemeris System (2020), NASA Solar System Dynamics Group http://ssd.jpl.nasa.gov/

21. ${ }^{21}$ Equatorial anisotropy in the inner part of Earth's inner core from autocorrelation of earthquake coda (2015), T. Wang et al https://doi.org/10.1038/ngeo2354

22. ${ }^{22}$ A complex dynamo inferred from the hemispheric dichotomy of Jupiter's magnetic field (2018), K. M. Moore et al https://doi.org/10.1038/s41586-018-0468-5

23. ${ }^{23}$ 10Be: Half-life and AMS-standards (1987), H. J. Hofmann et al https://doi.org/10.1016/0168-583X(87)90198-4

24. ${ }^{24} \mathrm{~A}$ new value for the half-life of $10 \mathrm{Be}$ by Heavy-Ion Elastic Recoil Detection and liquid scintillation counting (2010), G.Korschinek et al https://doi.org/10.1016/j.nimb.2009.09.020 
25. ${ }^{25}$ Rest Heart Rate and Life Expectancy (1997), H. J. Levine https://doi.org/10.1016/s0735-1097(97)00246-5

26. ${ }^{26}$ Complete Relativity: Nature of observables (2020), Amenoum https://amenoum.org/complete_relativity.html\#outref1

27. ${ }^{27}$ The species of homo (2020), Amenoum https://amenoum.org/log/25_species_of_homo.html

28. ${ }^{28}$ Athropogenic carbon and ocean pH, Nature 425, 365 (2003), K. Caldeira et al

29. ${ }^{29}$ Ocean acidification and the Permo-Triassic mass extinction (2014), M. O. Clarkson et al https://doi.org/10.1126/science.aaa2815

30. ${ }^{30}$ Essentials of geology, 4th edition, 256 (2013), S. Marshak

31. ${ }^{31}$ Characteristic disruptions of an excitable carbon cycle (2019), D. H. Rothman https://doi.org/10.1073/pnas.1905164116

32. ${ }^{32}$ Thresholds of catastrophe in the Earth system (2017), D. H. Rothman https://doi.org/10.1126/sciadv.1700906

33. ${ }^{33}$ Do Red and Green Make Brown?: Perspectives on Plastid Acquisitions within Chromalveolates (2011), R. G. Dorrell et al https://doi.org/10.1128/EC.00326-10

34. ${ }^{34}$ Future climate forcing potentially without precedent in the last 420 million years (2017), G. L. Foster et al https://doi.org/10.1038/ncomms14845

35. ${ }^{35}$ Periodicity of extinctions in the geologic past (1983), D. M. Raup et al https://dx.doi.org/10.1073/pnas.81.3.801

36. ${ }^{36}$ Periodic impact cratering and extinction events over the last 260 million years (2015), M. R. Rampino et al https://doi.org/10.1093/mnras/stv2088

37. ${ }^{37} \mathrm{CO} 2$ levels are rising (2018), Scripps Institution of Oceanography 
38. ${ }^{38}$ Understanding synchronicity (2020), Amenoum https://amenoum.org/log/19_understanding_synchronicity.html

39. ${ }^{39}$ Amenoum (2020), Amenoum https://amenoum.org/authors/Amenoum.html

40. ${ }^{40} 10 \mathrm{Be} \beta$ - Decay Evaluated Data (2019), TUNL Nuclear Data Evaluation Project https://tunl.duke.edu/sites/tunl.duke.edu/files/nucldata/GroundStatedecays/10Be.html

41. ${ }^{41}$ Where is the Earth's Radiogenic Helium? (1957), L. G. Carpenter et al https://www.nature.com/articles/179213a0

42. ${ }^{42}$ Periodic impact cratering and extinction events over the last 260 million years (2015), M. R. Rampino et al https://doi.org/10.1093/mnras/stv2088

43. ${ }^{43}$ Paleosol barometer indicates extreme fluctuations in atmospheric CO2 across the Cretaceous-Tertiary boundary (2002), L. Nordt et al https://doi.org/10.1130/0091-7613(2002)030\%3C0703:PBIEFI\%3E2.0.CO;2

44. ${ }^{44} \mathrm{~A}$ new value for the half-life of $10 \mathrm{Be}$ by Heavy-Ion Elastic Recoil Detection and liquid scintillation counting (2010), G. Korschinek et al https://doi.org/10.1016/j.nimb.2009.09.020

45. ${ }^{45}$ Dark matter's shadowy effect on Earth (2019), M. R. Rampino http://astronomy.com/magazine/2019/07/dark-matters--shadowy-effect--on-earth

46. ${ }^{46}$ Statistics of directional data (1972), K. V. Mardia

47. ${ }^{47}$ Periodic impact cratering and extinction events over the last 260 million years (2015), M. R. Rampino et al https://doi.org/10.1093/mnras/stv2088

48. ${ }^{48}$ Large igneous provinces and mass extinctions: An update (2014), D. Bond et al https://doi.org/10.1130/2014.2505\%2802\%29

49. ${ }^{49}$ Permo-Carboniferous Volcanism in Europe and North Africa: a Superplume exhaust valve in The Center of Pangea (1998), M. Doblas et al https://doi.org/10.1016/S0899-5362\%2897\%2900138-3 
50. ${ }^{50}$ Silurian Cycles: Linkages of Dynamic Stratigraphy with Atmospheric, Oceanic and Tectonic Changes (1998), L. Jeppsson

51. ${ }^{51}$ Discovery of a major negative $\delta 13 \mathrm{C}$ spike in the Carnian (Late Triassic) linked to the eruption of Wrangellia flood basalts (2012), J. Dal Corso et al https://doi.org/10.1130/g32473.1

52. ${ }^{52}$ The Silurian Mulde Event and a scenario for secundo - secundo events (2007), L. Jeppsson et al https://doi.org/10.1017/s0263593300000377

53. ${ }^{53}$ The Ireviken Event in the lower Silurian of Gotland, Sweden - relation to similar Palaeozoic and Proterozoic events (2003), A. Munnecke et al https://doi.org/10.1016/S0031-0182\%2803\%2900304-3

54. ${ }^{54}$ The Kalkarindji Large Igneous Province, Australia: Petrogenesis of the Oldest and Most Compositionally Homogenous Province of the Phanerozoic (2018), B. D. Ware et al https://doi.org/10.1093/petrology\%2Fegy040

55. ${ }^{55}$ Magmatic underplating beneath the Rajmahal Traps: Gravity signature and derived 3-D configuration (2004), A. P. Singh et al

56. ${ }^{56}$ A Concise Geologic Time Scale (2016), F. M. Gradstein et al

57. ${ }^{57}$ A Concise Geologic Time Scale (2016), F. M. Gradstein et al

58. ${ }^{58}$ Mantle Discontinuities (2017), A. Deuss http://www.geo.uu.nl/ deuss/research/discontinuities/

59. ${ }^{59}$ Imaging Mantle Heterogeneity with Upper Mantle Seismic Discontinuities (2015), N. Schmerr https://doi.org/10.1007/978-3-319-15627-9_3

60. ${ }^{60}$ Using the Baryonic Tully-Fisher Relation to Measure Ho (2020), J. Schombert et al https://doi.org/10.3847/1538-3881/ab9d88

61. ${ }^{61}$ Measurement of Jupiter's asymmetric gravity field (2018), L. less et al https://doi.org/10.1038/nature25776

62. ${ }^{62}$ Dynamics of Hippocampal Neurogenesis in Adult Humans (2013), Spalding et al https://dx.doi.org/10.1016/j.cell.2013.05.002 
63. ${ }^{63}$ Environmental Physiology of Animals (2009), P. Willmer

64. ${ }^{64}$ A Sceptics View: 'Kleiber's Law' or the '3/4 Rule' is neither a Law nor a Rule but Rather an Empirical Approximation (2014), A. J. Hulbert https://doi.org/10.3390/systems2020186

65. ${ }^{65}$ Tumor-derived TGF- $\beta$ inhibits mitochondrial respiration to suppress IFN-ץ production by human CD4+ T cells (2019), S. Dimeloe et al https://dx.doi.org/10.1126/scisignal.aav3334

66. ${ }^{66}$ Deadly 'two-faced' protein drives cancer growth, cripples T-cell avengers (2019), D. Ricks https://medicalxpress.com/news/2019-10-deadly-two-faced-protein-cancer-growth.html

67. ${ }^{67}$ UV surface brightness of galaxies from the local universe to $z \sim 5$ (2014), E. J. Lerner et al https://doi.org/10.1051/0004-6361/201936373

68. ${ }^{68}$ Evidence for anisotropy of cosmic acceleration (2019), J. Colin et al https://doi.org/10.1051/0004-6361/201936373

69. ${ }^{69}$ Atomic Radii in Crystals (1964), J. C. Slater https://doi.org/10.1063\%2F1.1725697

70. ${ }^{70}$ Covalent radii revisited (2008), B. Cordero et al https://doi.org/10.1039\%2Fb801115j

71. ${ }^{71}$ Experimental tests of Coulomb's Law and the photon rest mass (2004), L-C. Tu et al https://doi.org/10.1088/0026-1394/41/5/S04

72. ${ }^{72}$ The Pulse of the Solar Dynamo (2000), R. Howe et al http://soi.stanford.edu/press/GONG_MDI_03-00/

73. ${ }^{73}$ An Introduction to the Solar Tachocline (2007), D. Gough https://doi.org/10.1017/CBO9780511536243.002

74. ${ }^{74}$ Solar Interior Rotation and its Variation (2009), R. Howe https://doi.org/10.12942/Irsp-2009-1 